\title{
Extraction and Parametrization of Isobaric Trinucleon Elastic Cross Sections and Form Factors
}

\section{Scott Kevin Barcus}

William \& Mary - Arts \& Sciences, scottkbarcus@gmail.com

Follow this and additional works at: https://scholarworks.wm.edu/etd

Part of the Nuclear Commons

\section{Recommended Citation}

Barcus, Scott Kevin, "Extraction and Parametrization of Isobaric Trinucleon Elastic Cross Sections and Form Factors" (2019). Dissertations, Theses, and Masters Projects. William \& Mary. Paper 1563898943. http://dx.doi.org/10.21220/s2-zbjp-wp75

This Dissertation is brought to you for free and open access by the Theses, Dissertations, \& Master Projects at W\&M ScholarWorks. It has been accepted for inclusion in Dissertations, Theses, and Masters Projects by an authorized administrator of W\&M ScholarWorks. For more information, please contact scholarworks@wm.edu. 
Extraction and Parametrization of Isobaric Trinucleon Elastic Cross Sections and Form Factors

Scott Kevin Barcus

Shoreview, Minnesota

Master of Science, College of William \& Mary, 2014

Bachelor of Science, Drake University, 2012

A Dissertation presented to the Graduate Faculty of The College of William \& Mary in Candidacy for the

Degree of Doctor of Philosophy

Department of Physics

College of William \& Mary

May 2019 
(c) 2019

Scott Kevin Barcus

All Rights Reserved

Attribution-NonCommercial (CC BY-NC) 


\section{APPROVAL PAGE}

This Dissertation is submitted in partial fulfillment of the requirements for the degree of

Doctor of Philosophy

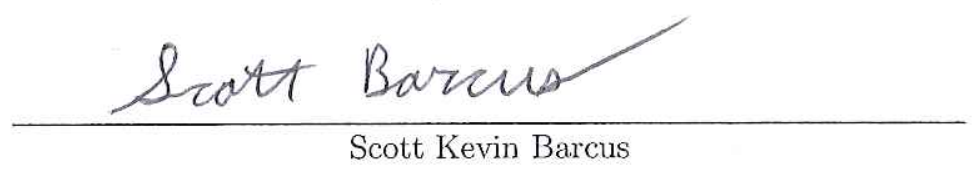

Approved by the Committee March 2019
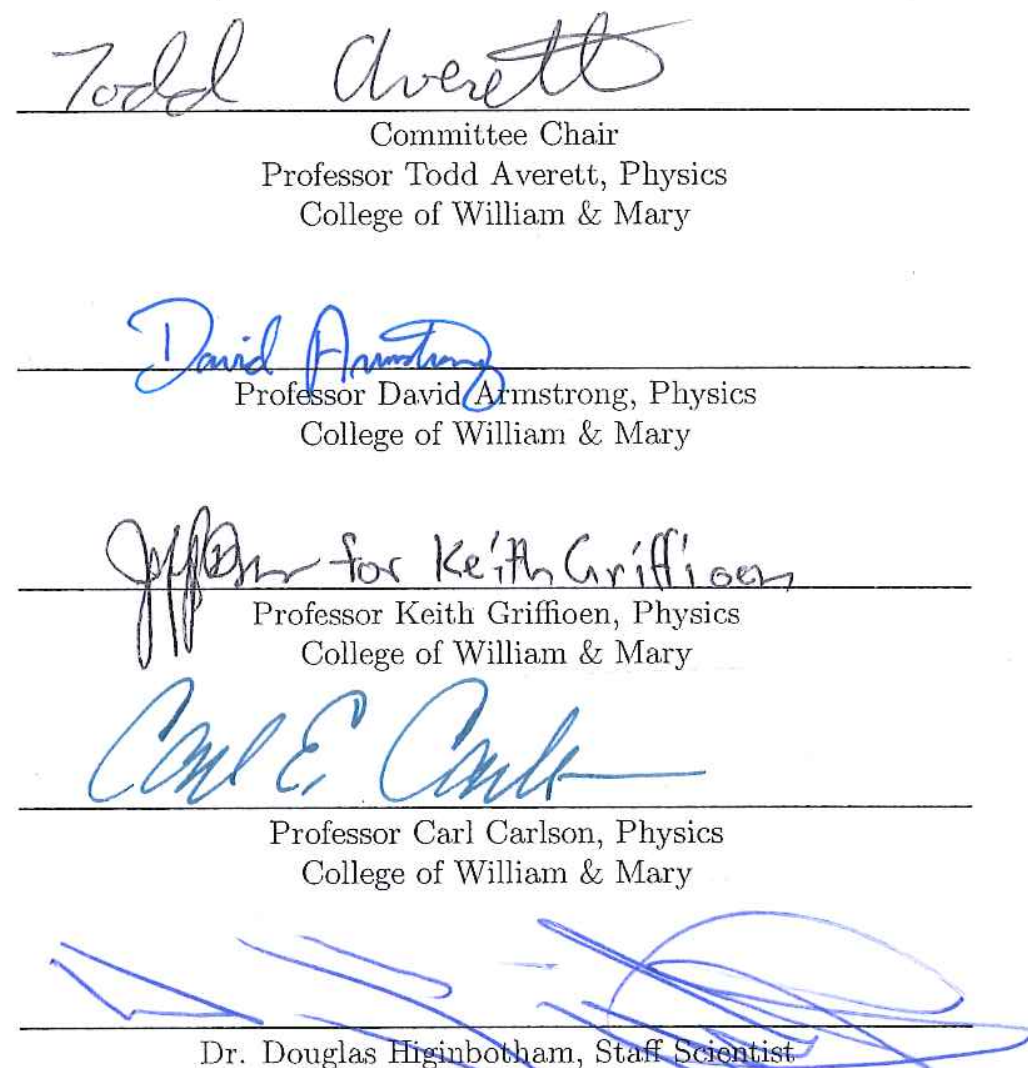

Dr. Douglas Higinbotham, Staff Scientist

Thomas Jefferson National-Accelerator Facility 


\section{ABSTRACT}

By mining data from Jefferson Lab Hall A experiment E08-014 a new ${ }^{3}$ He elastic cross section was extracted from a large quasielastic background. This measurement was taken with an initial beam energy of $3.356 \mathrm{GeV}$ and an angle of $20.51^{\circ}$. The cross section was found to be $1.345 \times 10^{-6} \mu \mathrm{b} / \mathrm{sr} \pm 0.086 \times 10^{-6} \mu \mathrm{b} / \mathrm{sr}$ at $Q^{2}=34.19 \mathrm{fm}^{-2}$. This new data point falls approximately halfway between the first and second diffractive minima of the ${ }^{3} \mathrm{He}$ form factors. When combined with recent high $Q^{2}{ }^{3} \mathrm{He}$ elastic cross section measurements from Jefferson Lab and MIT-Bates this new data point improves our knowledge of the cross section and form factors at large momentum transfers.

The new high $Q^{2}$ data motivate a reanalysis of the ${ }^{3} \mathrm{He}$ elastic cross section world data and provide an improved understanding of the magnetic form factor in particular. For this analysis the elastic cross section world data for ${ }^{3} \mathrm{He}$, and its mirror nuclei ${ }^{3} \mathrm{H}$, were collected. The world data spans a time frame from 1965 to 2016. The dataset contains electron energy ranges from tens of $\mathrm{MeV}$ to above $12 \mathrm{GeV}$ for measurements performed at many different facilities. The world data were then fit using a sum of Gaussians parametrization which allowed for the extraction of both targets' magnetic and electric form factors which were then used to calculate charge densities and radii.

The new charge and magnetic form factors for ${ }^{3} \mathrm{H}$ and the charge form factor for ${ }^{3} \mathrm{He}$ are in good agreement with the 1994 fits from Amroun et al. However, the addition of the new high $Q^{2}$ data has caused the ${ }^{3}$ He magnetic form factor's first diffractive minimum to shift up in $Q^{2}$ by $1-3 \mathrm{fm}^{-2}$ while also decreasing the magnitude of the magnetic form factor above $Q^{2} \approx 20 \mathrm{fm}^{-2}$. The first diffractive minima for ${ }^{3} \mathrm{H}$ are located at $Q^{2} \approx 13 \mathrm{fm}^{-2}$ and $Q^{2} \approx 23-24 \mathrm{fm}^{-2}$ for the charge and magnetic form factors respectively. The first diffractive minima for ${ }^{3} \mathrm{He}$ are located at $Q^{2} \approx 11 \mathrm{fm}^{-2}$ and $Q^{2} \approx 17-20 \mathrm{fm}^{-2}$ for the charge and magnetic form factors respectively.

The charge radius for ${ }^{3} \mathrm{He}$ was found to be $1.90 \mathrm{fm} \pm 0.00144 \mathrm{fm}$ in reasonable agreement with past measurements, and the charge radius for ${ }^{3} \mathrm{H}$ was found to be $2.02 \mathrm{fm} \pm 0.0133 \mathrm{fm}$ which is much larger than past measurements. However, each of these charge radii has an additional uncertainty that must be applied to them due to allowing all parameters to float freely during the sum of Gaussians fitting procedure. This additional uncertainty should be small for ${ }^{3} \mathrm{He}$, but it is likely quite significant for ${ }^{3} \mathrm{H}$ and would help bring this charge radius closer to agreement with past measurements that made different fitting choices. Unfortunately, this analysis was unable to quantify this additional uncertainty.

The new form factor fits were compared to modern theoretical predictions from the 2016 paper of Marcucci et al. The 'conventional' theoretical approach applied in this paper modelled two and three-body nucleon interactions with relativistic 
corrections and was reasonably successful at predicting the charge form factors of ${ }^{3} \mathrm{H}$ and ${ }^{3} \mathrm{He}$. $\chi$ EFT predictions were also often successful. However, while the 'conventional' approach still performed best, theory failed to accurately predict the magnetic form factors of either ${ }^{3} \mathrm{H}$ or ${ }^{3} \mathrm{He}$. The first diffractive minimum of the new ${ }^{3} \mathrm{He}$ magnetic form factor fits actually moved further away from theory. This disagreement between theory and experiment provides motivation for new asymmetry measurements using polarized ${ }^{3} \mathrm{He}$ and a polarized electron beam. When the beam is scanned in $Q^{2}$ on the target the sign of the asymmetry will flip at the form factor minima pinning down their true location. 


\section{TABLE OF CONTENTS}

Acknowledgements . . . . . . . . . . . . . . . . . . . . . iii

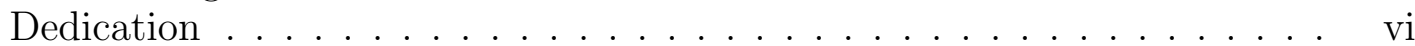

List of Tables . . . . . . . . . . . . . . . . . v vii

List of Figures . . . . . . . . . . . . . . . . . . . viii

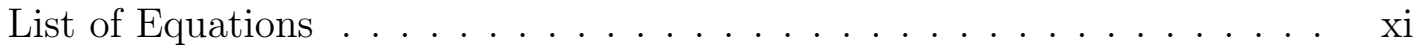

1 Elastic Electron Scattering . . . . . . . . . . . . . . . . . . . 1

1.1 Kinematics of Elastic Electron Scattering. . . . . . . . . . . . . 1

1.2 Differential Cross Section $\ldots \ldots \ldots \ldots \ldots$

1.3 Nuclear Form Factors. . . . . . . . . . . . . . . . . . . . . 5

1.4 Motivation and Mirror Nuclei . . . . . . . . . . . . . . . . . . 14

2 Experimental Setup . . . . . . . . . . . . . . . . . . . . . 17

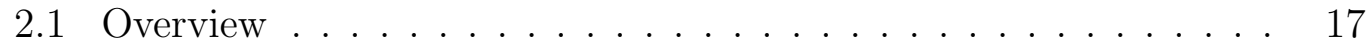

2.2 Experiment E08-014 . . . . . . . . . . . . . . . . . . . 17

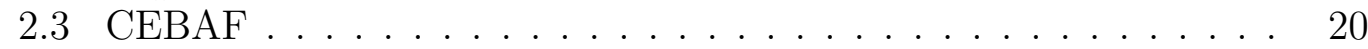

2.4 Hall A Beamline. . . . . . . . . . . . . . . . . . . . . . . . . . 22

2.4 .1 Beam Energy . . . . . . . . . . . . . . . . . . . . 23

2.4 .2 Beam Position Monitors . . . . . . . . . . . . . . . . . . 24

2.4 .3 Raster. . . . . . . . . . . . . . . . . . . . . . . 25

2.4 .4 Beam Current Monitors . . . . . . . . . . . . . . . . . 26

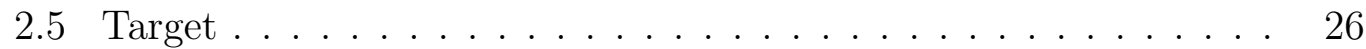

2.6 High Resolution Spectrometers . . . . . . . . . . . . . . . . . . 28

2.7 Detector Package $\ldots \ldots \ldots \ldots \ldots \ldots \ldots$

2.7 .1 Vertical Drift Chambers . . . . . . . . . . . . . . . . . 31

2.7 .2 Scintillator Counters . . . . . . . . . . . . . . . . . . . 37

2.7 .3 Gas Cherenkov . . . . . . . . . . . . . . . . . . . 38

2.7.4 Electromagnetic Calorimeters. . . . . . . . . . . . . . . . . 41

2.8 Data Acquisition System $\ldots \ldots \ldots \ldots \ldots \ldots$. . . . . . . . . . 42

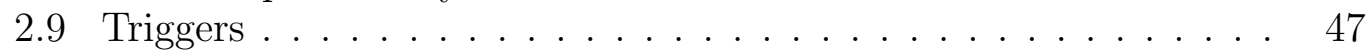

2.10 High Resolution Spectrometer Optics . . . . . . . . . . . . . . 47

2.10 .1 Hall A Coordinate Systems . . . . . . . . . . . . . . . . 47

2.10 .2 Spectrometer Optics Optimization Procedure . . . . . . . . 50

3 Cross Section Extraction . . . . . . . . . . . . . . . . . . . . 54

3.1 Overview . . . . . . . . . . . . . . . . . . . . . 54

3.2 Experimental Cross Section $\ldots \ldots \ldots \ldots \ldots \ldots \ldots$

3.3 Beam Charge $\ldots \ldots \ldots \ldots \ldots \ldots \ldots \ldots \ldots \ldots \ldots \ldots$

3.4 Corrections and Efficiencies $\ldots \ldots \ldots \ldots \ldots \ldots$

3.4 .1 Live-time Correction . . . . . . . . . . . . . . . . . . . 57

3.4 .2 Trigger Efficiency $\ldots \ldots \ldots \ldots \ldots \ldots \ldots$

3.4 .3 VDC Single Track Efficiency _. . . . . . . . . . . . . . 58

3.4 .4 Particle Identification $\ldots \ldots \ldots$. . . . . . . . . . . . . . . 60

3.4 .5 Gas Cherenkov Efficiency . . . . . . . . . . . . . . . . . 64

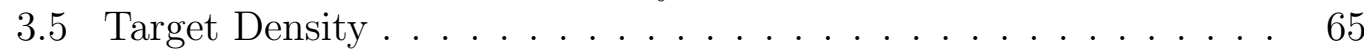

3.6 Cuts $\ldots \ldots \ldots \ldots \ldots \ldots$ 
$3.7{ }^{3} \mathrm{He}$ Elastic Cross Section Monte Carlo . . . . . . . . . . . . . . . 73

3.8 Radiative Corrections . . . . . . . . . . . . . . . . . . 74

3.9 Aluminum Background Subtraction . . . . . . . . . . . . . 78

3.10 Electron Yields to Cross Sections. . . . . . . . . . . . . . . . . 80

3.11 Bin Centering Corrections . . . . . . . . . . . . . . . . . 86

3.12 Uncertainty $\ldots \ldots \ldots \ldots$. . . . . . . . . . . . . . . . . . 89

3.13 Comparisons with Other Measurements . . . . . . . . . . . . . 97

4 Global Fits . . . . . . . . . . . . . . . . . . . . . . . . . . . . . . . 999

4.1 World Data. . . . . . . . . . . . . . . . . . . . . . . . . . . . . . . . . 99

4.2 Sum of Gaussians Parametrization . . . . . . . . . . . . . . . . 104

4.3 New SOG Fits . . . . . . . . . . . . . . . . . . . . . . . . . . . . 109

4.3 .1 Gaussian Radii Placement. . . . . . . . . . . . . . . . . . . 109

4.3 .2 Number of Gaussians. . . . . . . . . . . . . . . . . . . . . 112

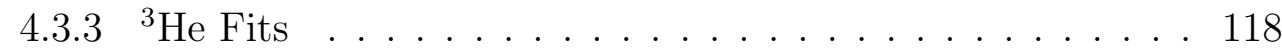

4.3 .4 Revisiting the E08-014 Cross Section . . . . . . . . . . . . 138

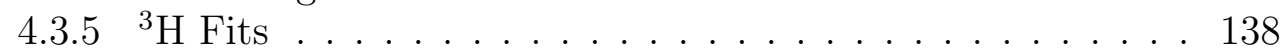

\begin{tabular}{|lll}
\hline 4.3.6 & 3 He Comparison with Theory and Previous Measurements & 154
\end{tabular}

4.3.7 ${ }^{3} \mathrm{H}$ Comparison with Theory and Previous Measurements . 159

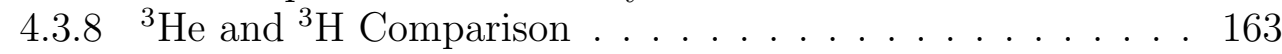

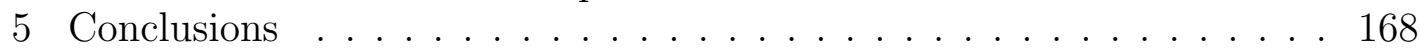

References . . . . . . . . . . . . . . . . . . . . . . . 172 


\section{ACKNOWLEDGEMENTS}

This thesis was made possible by the many extraordinary people in my life. Words are insufficient to express my gratitude to all of you.

Thank you to my advisor, Todd Averett, for his constant support and guidance. I am extremely grateful for the freedom and independence you allowed me in my work. The free reign you gave me at Jefferson Lab let me experience every part of planning, building, running, and analyzing experiments.

I would also like to thank my Jefferson Lab advisor, Douglas Higinbotham. You had no obligation to give me your time, yet you chose to become a mentor. In doing so, you gave me countless hours of advice and guidance. Your passion and enthusiasm for physics was a steady reminder of why we do our research. You kept advocating for my part of the Tritium Experiments, and though it never happened you provided me with the data used in this thesis making thesis plan number three the final one.

My thanks to the many others at Jefferson Lab who supported me in my graduate work. I am grateful to my other Jefferson Lab mentors, Bogdan Wojtsekhowski and Bob Michaels, for patiently lending me their time and expertise as I learned the work of a physics researcher. Thanks to all of the fellow graduate students I worked with over the years, particularly those in the Tritium Collaboration. I offer a special thank you to, Dien Nguyen, for pioneering this dataset and helping me with the initial analysis. Thanks also to the many postdocs I have worked with including Carlos Ayerbe-Gayoso, Huan Yao, Evan McClellan, and Florian Hauenstein for their willingness to teach me.

Thanks as well to Eugene Tracy and Seth Aubin, along with the other members of the Climate Steering Committee and the Diversity Advisory Committee. These committees provided me a constructive outlet and source of comfort in dark times. While our are efforts are just the beginning, and will likely never truly end, the department is better for all of your hard work.

Thank you to the staff working behind the scenes to make the physics department tick. The dedication to the department and students shown by Paula Perry, Eleonor Wilkinson, Carolyn Hankins, and Bonnie Willard makes our journey from orientation to graduation as smooth and well-organized as possible.

Too many people have contributed along my educational journey to list, but a few teachers made particularly large impacts on my life and education. At W\&M this includes Seth Aubin and Chris Carone for their outstanding teaching abilities. At Drake my professors Athanasios Petridis and Charles Nelson were excellent teachers as well, and Dr. Petridis recommended W\&M and Jefferson Lab to me. In high school my teachers Dan Butler, Gretchen Nesset, Graham 
Wright, and Cheryl Wason were phenomenal teachers in academics and life. I would also like to thank my elementary school science teacher John 'Jack' Briggs for sparking my interest in science with the star lab and crayfish labs as well as Margot Brooks, Fran Buesgens, and Ms. Bell.

Of course, none of this would be possible without the love and support of my family. Thank you to my parents, Kevin and Vicki Barcus, for giving me every possible advantage in life. Thank you for encouraging me to follow my own path in life and for cheering me on at every turn. You raised me to be the person I am happy to be today. I will always be proud to return your unconditional love. Thanks to my brother, Jeff Barcus, who has always been a kindred spirit and someone I can be completely open with. I will always cherish your companionship. Thanks also to my grandparents Nancy and Roger Johnson and Colleen and Wayne Barcus as well as my extended family for their love. I look forward to being back for more fishing trips and croquet games in the future.

I must also thank the many friends I made at W\&M. Without their help, passing my classes and completing this program would have been infinitely more difficult. To Matthew 'Perry' Nerem, our racquetball games and 'bro nights' are always a highlight of my week, and your friendship means the world to me. To Aria Johansen, you showed me how to live a strong life and opened my mind to a world of new possibilities. Your friendship taught me more about myself than anyone else has, and it will always be treasured. To Kurtis Bartlett, I have met none as steadfast, loyal, and principled as you. I do not have the room to tell you all how much you mean to me, but to Daniel Salmon, Carlie Crawford, Douglas Beringer, Melissa Beebe, Anashe Bandari, Marco Colo, Denise McKaig, and the others I met along the way thank you for your friendship. Few times were as happy as our board game days.

Finally, while I consider many of those above to be family, I want to thank my first chosen family as well. To Nathaniel Severson, since that first day we rode Big Wheels together your friendship has always been a source of joy for me. To Handi Xue, your decision that we would be friends in middle school was the best decision ever made for me. Growing up with the two of you formed me into the person I am today, and I am forever grateful for your friendship. To Evan Shapiro, you went from a randomly assigned roommate to a lifelong friend in almost no time at all. Ever since our late night dorm room chats you have always inspired me to better myself. To Forrest Williams, I can always count on you to laugh at a terrible joke, and I am so thankful for the ear you are always willing to lend when things get rough. Never have I had a friend more eager to pour their considerable energy into making the most of their friendships. To John Message, my first chosen roommate, I will always treasure the time we spent on the couch with me yelling at the news and you tolerating this in companionable silence. You taught me so much about being an adult and were instrumental in broadening my somewhat limited horizons. To Nicholas Dorando, 
my other chosen roommate, you imbue everyone around you with your unbridled enthusiasm for life and all of its joys. You make me and the world better by showing how one lives a life full of empathy and kindness. Thank you all for being there for me through the good and the bad, especially when I was probably a lot less fun to be around. Whether it is called the Aquarium, the Aviary, or the Apiary our regular calls have kept me sane throughout this process, and they give me a great sense of comfort knowing that I will never be alone going forward.

Thank you all! 
This dissertation is dedicated to my family and friends. 


\section{LIST OF TABLES}

3.1 BCM Calibration Constants for E08-014 . . . . . . . . . . . . 55

3.2 Charge Accumulated . . . . . . . . . . . . . . . . 56

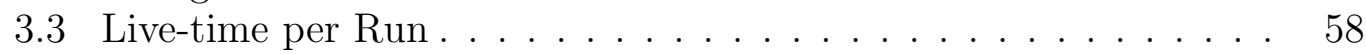

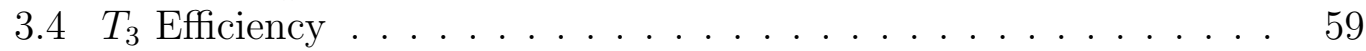

3.5 VDC Track Efficiency for HRSs . . . . . . . . . . . . . . . 60

3.6 Summary of Acceptance Cuts . . . . . . . . . . . . . . . . . . . . . . 69

3.7 Summary of Correction Factors . . . . . . . . . . . . . . . . . . 82

3.8 Combined Exponential and Gaussian Fit Parameters . . . . . . . . . 86

3.9 Bin Width at $3.356 \mathrm{GeV}$ and Spectrometer Setting of $21.04^{\circ}$. . . 88

3.10 Table of Uncertainties . . . . . . . . . . . . . . . . . . 90

4.1 Accumulated World Data for ${ }^{3} \mathrm{H}$ Elastic Scattering . . . . . . . . . 101

4.2 Accumulated World Data for ${ }^{3}$ He Elastic Scattering . . . . . . . . . 102

4.3 Determination of $N_{\text {Gaus }}$ for ${ }^{3} \mathrm{He}$. . . . . . . . . . . . . . . . . . . 115

4.4 Determination of $N_{\text {Gaus }}$ for ${ }^{3} \mathrm{H}$. . . . . . . . . . . . . . . . . 117

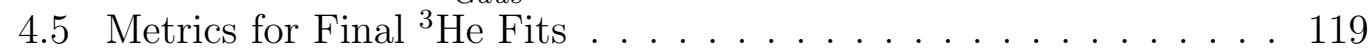

4.6 Parameters for ${ }^{3} \mathrm{He}$ World Data Representative Fit . . . . . . . . . . . 129

4.7 Metrics for ${ }^{3} \mathrm{He}$ World Data Representative Fit . . . . . . . . . . . . 131

4.8 Metrics for Final ${ }^{3}$ H Fits . . . . . . . . . . . . . . . . . . . . . . . . . 139

4.9 Parameters for ${ }^{3} \mathrm{H}$ World Data Representative Fit . . . . . . . . . 150

4.10 Metrics for ${ }^{3} \mathrm{H}$ World Data Representative Fit . . . . . . . . . . . . 150 


\section{LIST OF FIGURES}

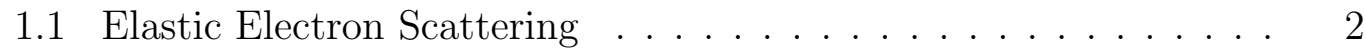

1.2 Hard Sphere Charge Distribution Form Factor. . . . . . . . . . . . 6

1.3 Rosenbluth Separtation $\ldots \ldots \ldots \ldots$. . . . . . . . . . . . . . . . . . . . 13

2.1 Thomas Jefferson National Accelerator Facility . . . . . . . . . . . 18

$2.2 \quad$ Kinematic Coverage of Experiment E08-014 . . . . . . . . . . . . . 19

$2.3 \quad$ Elastic Band for ${ }^{3} \mathrm{He} \ldots \ldots \ldots \ldots$. . . . . . . . . . . . 19

2.4 Elastic Peak in $x_{B j} \ldots \ldots \ldots \ldots \ldots \ldots$

2.5 Location of New Elastic Measurement . . . . . . . . . . . . . . . . . 21

2.6 Hall A Side View . . . . . . . . . . . . . . . . . . . . . . . . . . . . . . . . . . 22

2.7 Hall A Top View . . . . . . . . . . . . . . . . . . . . . . 23

2.8 Arc Energy Measurement Diagram. . . . . . . . . . . . . . . . . . 24

2.9 Layout of Hall A Beamline Components . . . . . . . . . . . . . . . 25

2.10 Target Ladder for Experiment E08-014 . . . . . . . . . . . . . . . . 27

2.11 Table of Target Information for Experiment E08-014 . . . . . . . . . 28

2.12 Cryogentic Monitoring and Control Screen . . . . . . . . . . . . . . 29

2.13 Side View of Single HRS . . . . . . . . . . . . . . . . . . . 30

2.14 Table of Important HRS Values . . . . . . . . . . . . . . . . . . . . . . . . . . . . . . . . . . . .

2.15 External VDC Diagram . . . . . . . . . . . . . . . . . . . 32

2.16 Internal VDC Side View Diagram . . . . . . . . . . . . . . . . . 33

2.17 Example Wire Efficiency Spectra . . . . . . . . . . . . . . . . . . . . . . . 34

2.18 Wire Timing Spectrum . . . . . . . . . . . . . . . . . . . . . . . . . . 35

2.19 Trajectory Reconstruction . . . . . . . . . . . . . . . . . . . . . . 36

2.20 Single Plastic Scintillator Paddle . . . . . . . . . . . . . . . . . . . . . . . . . . . 38

2.21 EM Shock Wave . . . . . . . . . . . . . . . . . . . . . . . . . . . 39

2.22 Hall A GC Interior . . . . . . . . . . . . . . . . . . . . . . . 40

2.23 Hall A Electromagnetic Calorimeters . . . . . . . . . . . . . . . . . . . . 42

2.24 Schematic of Hall A DAQ and Trigger System . . . . . . . . . . . . . . . 43

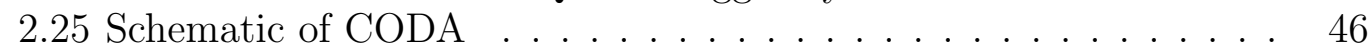

2.26 Hall Coordinate System . . . . . . . . . . . . . . . . . . . . 48

2.27 Target Coordinate System . . . . . . . . . . . . . . . . . . . . 49

2.28 Detector Coordinate System . . . . . . . . . . . . . . . . . . 50

2.29 Transport Coordinate System . . . . . . . . . . . . . . . . . . . 50

2.30 Focal Plane Coordinate System . . . . . . . . . . . . . . . . . . . . . . . . . . . . . . 51

2.31 Optics Sieve Plate for E08-014 . . . . . . . . . . . . . . . . . . . . . . . . 52

3.1 BCM Readouts for Run 4074 . . . . . . . . . . . . . . . . . . . . . 57

3.2 T3 Efficiency by Run . . . . . . . . . . . . . . . . . . . . . . . . . . 59

$3.3 \quad$ PID with the Pion Rejectors . . . . . . . . . . . . . . . . . . . . 61

3.4 PID with the Gas Cherenkov . . . . . . . . . . . . . . . . . . . 62

3.5 PID with the Pion Rejectors . . . . . . . . . . . . . . . . 63

3.6 PID with the Gas Cherenkov . . . . . . . . . . . . . . . . . . . . 64

3.7 GC Efficiency . . . . . . . . . . . . . . . . . . . . . 65

$3.8{ }^{3}$ He Boiling Effect . . . . . . . . . . . . . . . . . . . . . 67

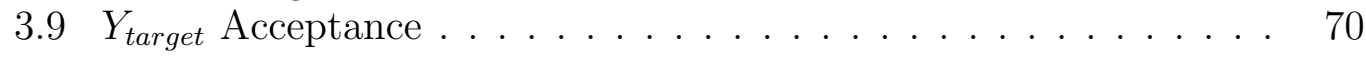


$3.10 \theta$ Acceptance . . . . . . . . . . . . . . . . . . . . . . 71

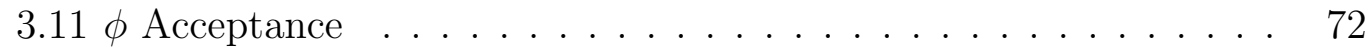

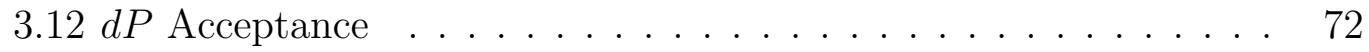

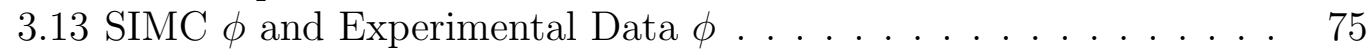

3.14 SIMC $\theta$ and Experimental Data $\theta \ldots \ldots . \ldots . \ldots . . . \ldots 75$

3.15 SIMC $d P$ and Experimental Data $d P \ldots \ldots . \ldots . \ldots . \ldots 76$

3.16 Born and Lowest Order Radiative Correction Diagrams . . . . . . 77

3.17 Scaled Aluminum Background . . . . . . . . . . . . . . . . . . . 79

3.18 Combined Fit of $x_{B j}$ for E08-014 . . . . . . . . . . . . . . . . . . . . 82

3.19 SIMC Elastically Scattered Electrons . . . . . . . . . . . . . . . 83

3.20 Histogram Binned to Fit of Quasielastic Background . . . . . . . . . 84

3.21 Elastic Peak Fits of ${ }^{3} \mathrm{He}$ Production Runs and SIMC Elastics

Summed with QE Background Fit . . . . . . . . . . . . . . 85

$3.22{ }^{3} \mathrm{He}$ Elastic Cross Section at $E_{0}=3.356 \mathrm{GeV}$. . . . . . . . . . 87

$3.23{ }^{3} \mathrm{He}$ Elastic Cross Section $Q^{2}$ Bin at $E_{0} 3.356 \mathrm{GeV}$. . . . . . . . . . 89

3.24 Optics Sieve Monte Carlo . . . . . . . . . . . . . . . . . . . . . 93

3.25 Optics Sieve Monte Carlo Areas Histogram . . . . . . . . . . . . . . 94

3.26 Solid Angle Acceptance of ${ }^{3} \mathrm{He}$ Cell in $Y_{\text {target }}$. . . . . . . . . . . . . . 95

4.1 Individual Gaussians of the ${ }^{3} \mathrm{He}$ Charge Density . . . . . . . . . . . . . 107

$4.2{ }^{3}$ He Charge Form Factors $\ldots \ldots \ldots$. . . . . . . . . . . . . . . . 121

$4.3{ }^{3}$ He Magnetic Form Factors . . . . . . . . . . . . . . . . . . . . . . . . . 123

$4.4{ }^{3}$ He Charge Densities $\ldots \ldots \ldots$. . . . . . . . . . . . . . . . . . . . 124

$4.5{ }^{3} \mathrm{He}$ RMS Charge Radii . . . . . . . . . . . . . . . . . . . . . . . 126

$4.6{ }^{3} \mathrm{He} Q_{i}$ Fit Parameters $\ldots \ldots \ldots \ldots$. . . . . . . . . . . . . . . . . . . 128

$4.7{ }^{3} \mathrm{He} R_{i}$ Values and Separations . . . . . . . . . . . . . . . 130

$4.8{ }^{3}$ He Form Factors for the New Representative Fit of World Data. 132

$4.9{ }^{3}$ He Representative Form Factors with World Data and Amroun Fitl 133

$4.10^{3} \mathrm{He}$ Cross Section World Data Distribution . . . . . . . . . . . 134

$4.11{ }^{3}$ He Representative Fit $\chi^{2}$ vs. $Q^{2}$. . . . . . . . . . . . . . . . . . 135

$4.12{ }^{3} \mathrm{He}$ Representative Fit Residual vs. $Q^{2}$. . . . . . . . . . . . . . 136

$4.13^{3}$ He Elastic Cross Section $\ldots \ldots \ldots$. . . . . . . . . . . . . . . . . . . . . . . . . . . . . . . .

$4.14{ }^{3} \mathrm{H}$ Charge Form Factors . . . . . . . . . . . . . . . . . . . . . . . . . . . . . . . . . . . . 141

$4.15^{3} \mathrm{H}$ Magnetic Form Factors . . . . . . . . . . . . . . . . . . . . . . . . . . . . 143

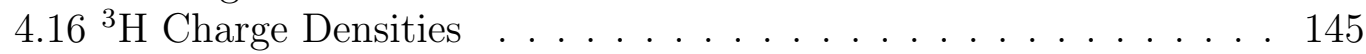

$4.17^{3} \mathrm{H}$ RMS Charge Radii . . . . . . . . . . . . . . . . . . . . . 146

$4.18{ }^{3} \mathrm{H} Q_{i}$ Fit Parameters . . . . . . . . . . . . . . . . . . . . . . . . . . 148

$4.19{ }^{3} \mathrm{He} R_{i}$ Values and Separations . . . . . . . . . . . . . . . . 149

$4.20{ }^{3} \mathrm{H}$ Form Factors for the New Representative Fit of World Data . 151

$4.21^{3} \mathrm{H}$ Representative Form Factors with World Data and Amroun Fit 152

$4.22{ }^{3} \mathrm{H}$ Cross Section World Data Distribution . . . . . . . . . . . 153

$4.23{ }^{3}$ H Representative Fit $\chi^{2}$ vs. $Q^{2} \ldots \ldots \ldots$. . . . . . . . . . 153

$4.24{ }^{3}$ H Representative Fit Residual vs. $Q^{2}$. . . . . . . . . . . . . . 154

$4.25{ }^{3} \mathrm{He} F_{c h}$ Comparison of Results $\ldots \ldots \ldots$. . . . . . . . . . . . 156

$4.26{ }^{3} \mathrm{He} F_{m}$ Comparison of Results . . . . . . . . . . . . . . . . 157

$4.27^{3} \mathrm{He} F_{c h}$ at $Q^{2}=0$. . . . . . . . . . . . . . . . . . . . . . 159

$4.28{ }^{3} \mathrm{H} F_{c h}$ Comparison of Results . . . . . . . . . . . . . . 160 
$4.29{ }^{3} \mathrm{H} F_{m}$ Comparison of Results . . . . . . . . . . . . . . . 161

$4.30{ }^{3} \mathrm{H} F_{c h}$ at $Q^{2}=0 \ldots \ldots \ldots \ldots \ldots \ldots \ldots \ldots \ldots$

$4.31 F_{c h}$ Comparison $\ldots \ldots \ldots \ldots \ldots \ldots \ldots \ldots$

$4.32 F_{m}$ Comparison $\ldots \ldots \ldots \ldots \ldots \ldots \ldots \ldots \ldots$

4.33 Charge Density Comparison . . . . . . . . . . . . . . . 166 


\section{LIST OF EQUATIONS}

$1.1 \quad$ Elastically Scattered Electron Energy (E') . . . . . . . . . . . . . . . 2

1.2 Energy Lost by Incident Electron $(\nu)(\ldots \ldots \ldots \ldots \ldots$

1.3 Four-Momentum Transfer $\left(Q^{2}\right) \ldots \ldots \ldots \ldots \ldots \ldots$

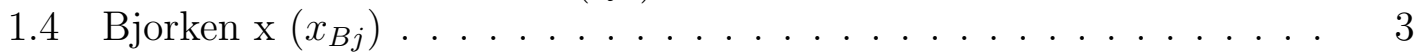

1.5 Rutherford Scattering as a Function of Angle . . . . . . . . . . . . 3

1.6 Rutherford Scattering as a Function of Momentum Transfer . . . . . 4

1.7 Mott Equation No Recoil with Relativistic Term . . . . . . . . . . . 4

1.8 Mott Equation No Recoil Explicit . . . . . . . . . . . . . . . . . 4

1.9 Form Factor Term in Cross Section . . . . . . . . . . . . . . . 5

1.10 Form Factor as a Fourier Transform . . . . . . . . . . . . . 5

1.11 Charge Density as an Inverse Fourier Transform . . . . . . . . . 5

1.12 Approximate Charge Radius of a Hard Sphere of Charge . . . . . . 7

1.13 Small Electron Wavelength Approximation $\ldots \ldots \ldots \ldots . \ldots .7$

1.14 Cosine Taylor Expansion $\ldots \ldots \ldots \ldots \ldots$

1.15 Form Factor (3D Integral) $\ldots \ldots \ldots \ldots \ldots \ldots$

1.16 Form Factor (Radial) $\quad \ldots \ldots \ldots \ldots$

1.17 Mean Square Charge Radius . . . . . . . . . . . . . . . . . . 8

1.18 Form Factor relation to Mean Charge Radius . . . . . . . . . . . . . 8

1.19 Mean Charge Radius from Form Factor First Derivative . . . . . . 8

1.20 Planck-Einstein Relation $\ldots \ldots \ldots \ldots$

1.21 Wavelength $\ldots \ldots \ldots \ldots \ldots \ldots$. . . . . . . . . . . . . 8

1.22 Energy $\ldots \ldots \ldots \ldots \ldots \ldots \ldots$

1.23 Recoil Term . . . . . . . . . . . . . . . . . . . . . . . . . 9 9

1.24 Mott Equation adding Recoil . . . . . . . . . . . . . . . . . . . 9

1.25 Mott Equation $\ldots \ldots \ldots \ldots$. . . . . . . . . . . . . . . . . . 9

1.26 Magnetic Moment . . . . . . . . . . . . . . . . . . . . . . . 10

1.27 Cross Section Magnetic Term . . . . . . . . . . . . . . . . . 10

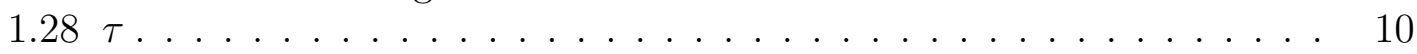

1.29 Proton and Neutron Magnetic Moments . . . . . . . . . . . . . . 10

1.30 Nuclear Magneton . . . . . . . . . . . . . . . . . . . . . . . . . . . 11

1.31 Rosenbluth Equation with Sach's Form Factors . . . . . . . . . . . . 11

1.32 Proton Sach's Form Factors $\ldots \ldots \ldots \ldots \ldots$

1.33 Neutron Sach's Form Factors . . . . . . . . . . . . . . . . . . . 11

1.34 Dirac Form Factor . . . . . . . . . . . . . . . . . . . . . . . 12

1.35 Pauli Form Factor . . . . . . . . . . . . . . . . . . . . . . . 12

1.36 Charge Form Factor $\left(\mathrm{F}_{c h}\right) \ldots \ldots \ldots \ldots \ldots \ldots$

1.37 Magnetic Form Factor $\left(\mathrm{F}_{m}\right) \quad \ldots \ldots \ldots \ldots \ldots \ldots \ldots$

1.38 Rosenbluth Equation $\ldots \ldots \ldots \ldots \ldots$

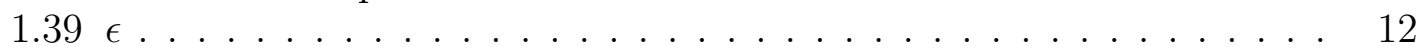

1.40 Reduced Cross Section. . . . . . . . . . . . . . . . . . . . 13

1.41 Binding Energy of a Nucleus . . . . . . . . . . . . . . . . . . 15

2.1 Beam Momentum (Arc Method) . . . . . . . . . . . . . . . . . . 24

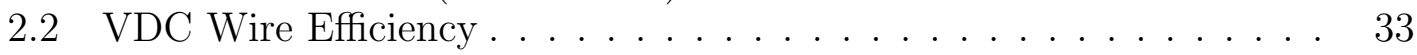


$2.3 \quad$ VDC Drift Distance $\ldots \ldots \ldots \ldots \ldots \ldots \ldots \ldots \ldots \ldots$

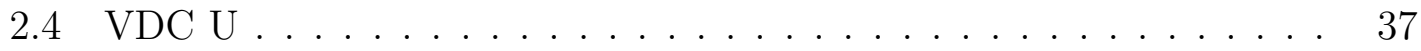

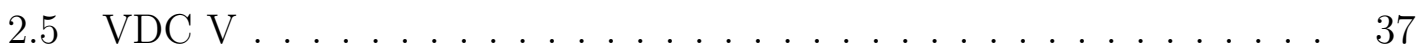

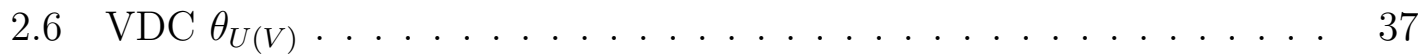

2.7 Gas Cherenkov Velocity Threshold $\ldots \ldots \ldots \ldots$

2.8 Cherenkov Shock Wave Angle $\ldots \ldots \ldots \ldots$

2.9 Gas Cherenkov Momentum Threshold . . . . . . . . . . . . . . . . . . 39

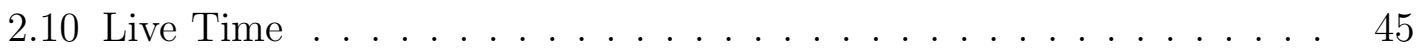

2.11 Momentum Fraction $(\mathrm{dP}) \ldots \ldots \ldots \ldots \ldots \ldots$

2.12 Optics Matrix . . . . . . . . . . . . . . . . . . . . . . 51

2.13 Momentum Fraction $(\delta) \ldots \ldots \ldots \ldots \ldots \ldots \ldots$

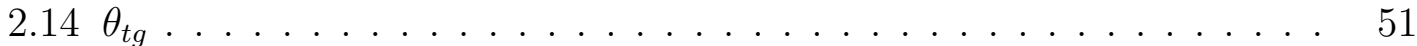

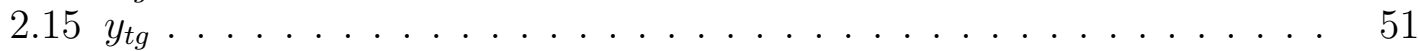

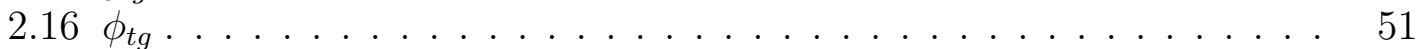

2.17 Optics Tensors $\left(D_{j, k, l}\right) \ldots \ldots \ldots \ldots \ldots \ldots \ldots \ldots \ldots \ldots \ldots \ldots \ldots$

2.18 Interaction Vertex $\left(Z_{\text {react }}\right) \quad \ldots \ldots \ldots \ldots \ldots \ldots \ldots \ldots$

2.19 Horizontal Sieve Position $\left(x_{\text {sieve }}\right) \quad \ldots \ldots \ldots \ldots \ldots$. . . . . . . 53

2.20 Vertical Sieve Position $\left.\left(y_{\text {sieve }}\right)\right] \ldots \ldots \ldots \ldots \ldots \ldots$

$3.1 \quad$ Experimental Cross Section $\ldots \ldots \ldots \ldots \ldots \ldots$

3.2 BCM Calibration $\ldots \ldots \ldots \ldots \ldots \ldots \ldots \ldots \ldots \ldots \ldots$

3.3 Beam Charge . . . . . . . . . . . . . . . . . . . 56

3.4 Number of Incident Electrons $\ldots \ldots \ldots \ldots \ldots$

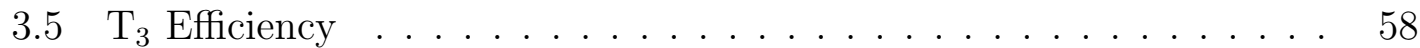

$3.6 \quad$ VDC Efficiency $\ldots \ldots \ldots \ldots \ldots \ldots \ldots$

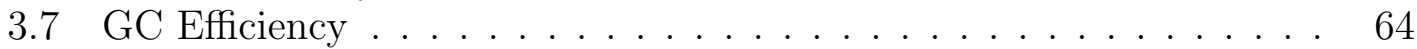

3.8 Fractional Target Density $\ldots \ldots \ldots \ldots \ldots . \ldots \ldots$

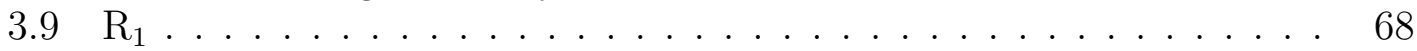

$3.10{ }^{4} \mathrm{He}$ Target Density Conversion to ${ }^{3} \mathrm{He}$ Target Density . . . . . . . . . 68

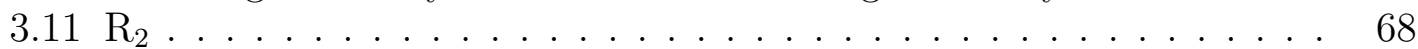

$3.12{ }^{3} \mathrm{He}$ Target Density . . . . . . . . . . . . . . . . . . . . . . . . . . . . 69

3.13 Radiative Corrections . . . . . . . . . . . . . . . . . . 78

3.14 Exponential Fit . . . . . . . . . . . . . . . . . . . . . . . 80

3.15 Gaussian Fit. . . . . . . . . . . . . . . . . . . . . . . . . . 81

3.16 Combined Exponential and Gaussian Fit. . . . . . . . . . . . . 81

3.17 Elastic Electron Yield Uncertainty $\ldots \ldots \ldots$. . . . . . . . . . . . . 91

3.18 GC Uncertainty . . . . . . . . . . . . . . . . . . . . . . . . . . . 94

3.19 Live Time Uncertainty. . . . . . . . . . . . . . . . . . . . . . . . 97

4.1 SOG Charge Density (No Tails) $\ldots \ldots \ldots \ldots \ldots \ldots$. . . . . . . 106

4.2 SOG Charge Density (Tails) $\ldots \ldots \ldots \ldots \ldots \ldots$

4.3 Charge Density Normalization . . . . . . . . . . . . . . . . . 107

4.4 SOG Form Factors . . . . . . . . . . . . . . . . . . . . . . . . . 108

4.5 SOG Cross Section . . . . . . . . . . . . . . . . . . . . . . . 108

$4.6 \mathrm{GeV}^{2}$ to $\mathrm{fm}^{-2}$ Conversion . . . . . . . . . . . . . . . . . . . . . . 109

$4.7 \quad Q^{2}$-Effective . . . . . . . . . . . . . . . . . . . . . . . . . 109

4.8 Electron Three Momentum . . . . . . . . . . . . . . . . . . . . . . . . . 109

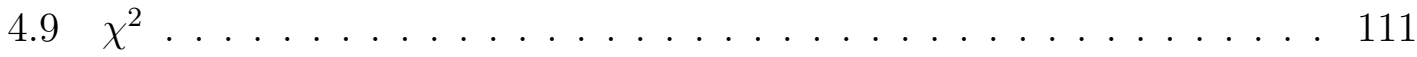


4.10 Reduced $\chi^{2} \ldots \ldots \ldots \ldots \ldots \ldots \ldots$

4.11 Akaike Information Criterion . . . . . . . . . . . . . . . . . . . 113

4.12 Bayesian Information Criterion . . . . . . . . . . . . . . . . . . . . . . . . . . . . . . . . . . . . . . .

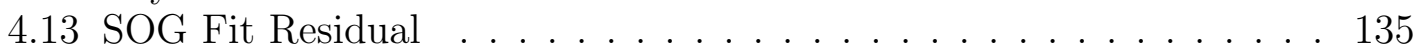

5.1 Asymmetry Measurement . . . . . . . . . . . . . . . . . . 171 


\section{Chapter 1}

\section{Elastic Electron Scattering}

Electron scattering is one of the most powerful tools available to physicists to study the nature of nuclear matter. When electrons are accelerated to high energies by means of a particle accelerator, like Jefferson Lab's CEBAF, and fired at a nuclear target the electrons scatter according to the nuclear structure of the target. This scattering is well described by quantum electrodynamics (QED). Thus, by measuring the scattered electrons (and occasionally other particles), called 'semi-inclusive electron scattering', the nuclear structure of the target is revealed.

\subsection{Kinematics of Elastic Electron Scattering}

The process of elastic electron scattering via the electromagnetic process is shown in Figure 1.1. An incident electron, with four-momentum $k=\left(E_{0}, \boldsymbol{k}\right)$, exchanges a virtual photon, $q=(\nu, \boldsymbol{q})$, with a target in the target's rest frame, $p=(M, 0)$. The virtual photon exchanges energy and momentum causing the electron to scatter with a scattering angle $\theta$ and four-momentum $k^{\prime}=\left(E^{\prime}, \boldsymbol{k}^{\prime}\right)$. The proton is also scattered with four-momentum $p^{\prime}$, but the proton is not measured in inclusive electron scattering. When the kinetic energy of this scattering process is conserved the process is called 'elastic'.

When the scattering process is elastic the entire process can be described by two 


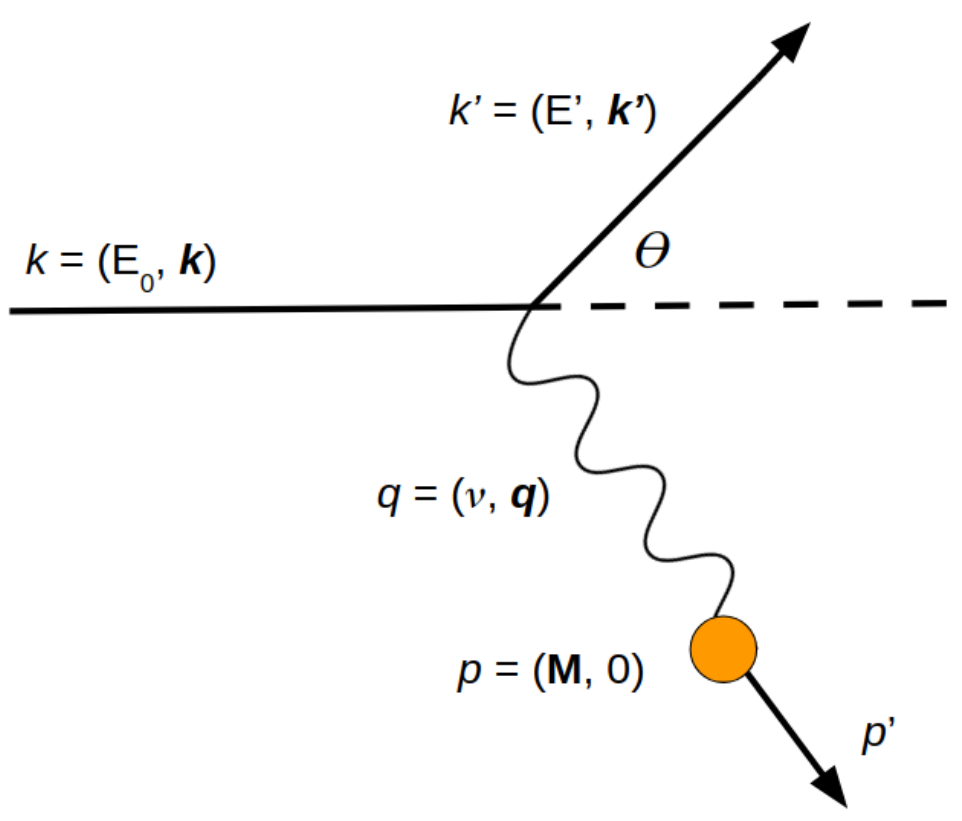

Figure 1.1: Elastic Electron Scattering. An incident electron interacts with a target by exchanging a virtual photon causing the electron to scatter.

variables. These variables are the scattering angle, $\theta$, and the initial energy, $E_{0}$. By using conservation of energy and momentum as well as applying the Einstein relation the scattered electron's final energy, $E^{\prime}$, is found to be given by Equation 1.1. The energy lost by the incident electron during scattering, $\nu$, is given by Equation 1.2 . The strength of the interaction, how much four-momentum is transferred to the target by the electron, is generally described as in Equation 1.3 . $Q^{2}$ is given in units of $(\mathrm{GeV} / \mathrm{c})^{2}$ which can be converted to $\mathrm{fm}^{-2}$ by multiplying the $(\mathrm{GeV} / \mathrm{c})^{2}$ result by a value of $\approx$ 25.7 .

$$
\begin{gathered}
E^{\prime}=\frac{E_{0}}{1+\frac{2 E_{0}}{M} \sin ^{2}\left(\frac{\theta}{2}\right)} \\
\nu=E_{0}-E^{\prime}
\end{gathered}
$$




$$
Q^{2}=-q^{2}=4 E_{0} E^{\prime} \sin ^{2}\left(\frac{\theta}{2}\right)
$$

Another useful quantity to define is Bjorken $x$, denoted $x_{B j}$, given by Equation 1.4. This variable is interpretable as the fraction of the nucleon's three-momentum carried by the quark struck by the electron in the Breit frame. For a single nucleon $0 \leq x_{B j} \leq 1$. However, for a nucleus $0 \leq x_{B j} \leq A$, where $A$ is the atomic mass number of the target. The elastic peak can then be found at $x_{B j} \approx A$. Taking ${ }^{3} \mathrm{He}$ as an example one would then expect to find the elastic peak at $x_{B j}=3$.

$$
x_{B j}=\frac{Q^{2}}{2 M\left(E_{0}-E^{\prime}\right)}
$$

\subsection{Differential Cross Section}

The differential cross section is proportional to the probability that an electron incident on a target will interact with that target. This can be thought of as the 'size' of the interaction. However, for a scattered electron to be measured it must be seen by a detector which measures within some acceptance $d \Omega$. Therefore, the differential cross section measures the probability that an electron will be scattered from a target into solid angle $d \Omega$.

Following the discussion of cross sections and form factors laid out in chapters five and six of [1], and largely adopting their notation, let us begin with the classical case of particles scattering from a fixed target nuclei. The Rutherford scattering equation can be obtained classically as Equation 1.5 or through non-relativistic quantum mechanics by assuming that the wave functions of the electron are plane waves (Born approximation).

$$
\left(\frac{d \sigma}{d \Omega}\right)_{\text {Rutherford }}=\frac{(z e Z e)^{2}}{\left(4 \pi \epsilon_{0}\right)^{2}\left(4 E_{0}\right)^{2} \sin ^{4}(\theta / 2)}
$$

Here $z(Z)$ is the atomic number of the incident particle (target), $e$ is the elementary 
charge, $\epsilon_{0}$ is the vacuum permittivity, and $E_{0}$ is the incident particle's initial energy. The Rutherford equation can also be written as Equation 1.6 following the quantum mechanical derivation, where $\alpha$ is the fine structure constant, $\hbar$ is the reduced Planck's constant, and $c$ is the speed of light. Notice that the cross section falls off like $\frac{1}{q^{4}}$ indicating that the interaction probability falls off rapidly with increased momentum transfer [1.

$$
\left(\frac{d \sigma}{d \Omega}\right)_{\text {Rutherford }}=\frac{4 Z^{2} \alpha^{2}(\hbar c)^{2} E^{\prime 2}}{|q c|^{4}}
$$

The Rutherford equation does not account for relativity, spin, or target recoil. To begin accounting for these quantities let us continue the derivation by adding relativity. To do this we add a second term to the cross section that is scaled by a constant and introduces angular dependence to Equation 1.6. This yields the preliminary version of the Mott equation while still neglecting recoil as seen in Equation 1.7.

$$
\left(\frac{d \sigma}{d \Omega}\right)_{\substack{\text { Mott } \\ \text { No Recoil }}}=\left(\frac{d \sigma}{d \Omega}\right)_{\text {Rutherford }}\left(1-\beta^{2} \sin ^{2}\left(\frac{\theta}{2}\right)\right)
$$

Here $\beta=\frac{v}{c}$ with $v$ being the velocity of the incident particle [1].

By taking the incident particle's velocity to its maximum value of $c$ we see $\beta$ goes to unity. Then Equation 1.7 simplifies via trigonometric identity to Equation 1.8 .

$$
\left(\frac{d \sigma}{d \Omega}\right)_{\substack{\text { Mott } \\ \text { No Recoil }}}=\left(\frac{d \sigma}{d \Omega}\right)_{\text {Ruth. }} \cos ^{2}\left(\frac{\theta}{2}\right)=\left(\frac{d \sigma}{d \Omega}\right)_{\text {Ruth. }}=\frac{4 Z^{2} \alpha^{2}(\hbar c)^{2} E^{\prime 2}}{|q c|^{4}} \cos ^{2}\left(\frac{\theta}{2}\right)
$$

Equation 1.8 now accounts for relativity, but also accounts for spin by suppressing scattering through $180^{\circ}$ for a spinless target which is forbidden by conservation of helicity 1]. 


\subsection{Nuclear Form Factors}

So far we have only considered scattering off of a pointlike target. Real nuclear targets are made up of atoms with differing geometry. To quantize the spatial extent of a target we introduce the concept of form factors. Form factors contain all of the transverse spatial information about the target. Multiplying the Mott cross section, excepting recoil, by the form factor we get the experimental cross section as in Equation 1.9. By measuring the experimental cross section of a target at various angles for a single energy and dividing out the Mott cross section the form factor, $F\left(q^{2}\right)$, of a target can be determined.

$$
\left(\frac{d \sigma}{d \Omega}\right)_{\exp }=\left(\frac{d \sigma}{d \Omega}\right)_{\text {No Recoil }}^{\text {Mott }}\left|F\left(q^{2}\right)\right|^{2}
$$

Continuing to assume that recoil is negligible as well as the validity of the Born approximation the target's form factors can be written as the Fourier transform of a charge distribution, $\rho(x)$, as in Equation 1.10. If the charge distribution is spherically symmetric then the form factor equals the right side of Equation 1.10 with the integral of $\rho(r)$ normalized to unity.

$$
F\left(q^{2}\right)=\int e^{\frac{i q \cdot x}{\hbar}} \rho(x) d^{3} x \stackrel{x \rightarrow r}{\longrightarrow} 4 \pi \int \rho(r) \frac{\sin (|q| r / \hbar)}{|q| r / \hbar} r^{2} d r
$$

This procedure can be inverted to find the charge distribution of a target from its form factor as in Equation 1.11 ].

$$
\rho(r)=\frac{1}{(2 \pi)^{3}} \int F\left(q^{2}\right) e^{\frac{-i q \cdot x}{\hbar}} d^{3} q
$$

Let us now examine a simple example of a charge distribution and its form factor. Assume that there is a charge distribution in the shape of a hard sphere, i.e. a solid ball of constant charge density that drops to zero beyond a certain radius. This is a reasonable first order model for the charge distribution of an atom. The top plot in Figure 1.2 shows a hard sphere of charge density. The bottom plot shows the form 
factor, Fourier transform, of the upper hard sphere plot. Now we can see that the form factor of a hard sphere distribution of charge yields an oscillatory and decreasing form factor [1].
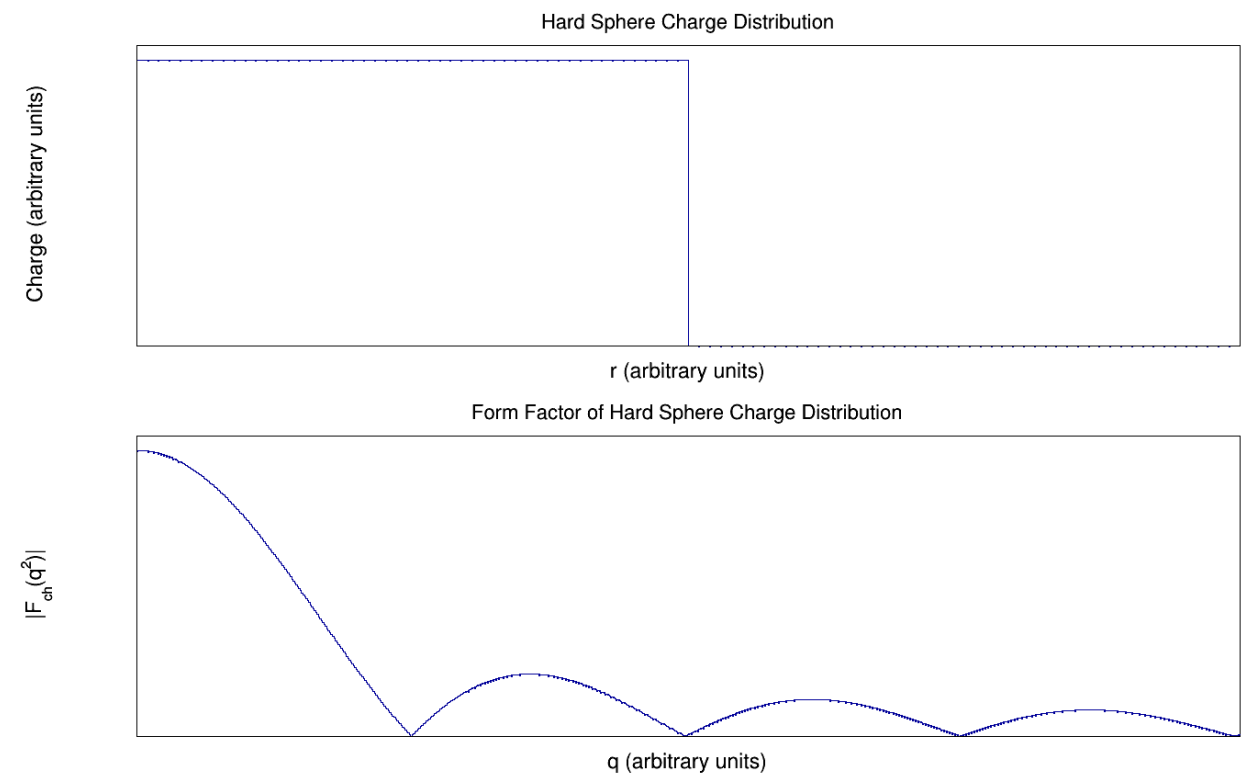

Figure 1.2: Hard Sphere Charge Distribution Form Factor. Taking the Fourier transform of a hard sphere charge distribution (top plot) yields an oscillatory form factor (bottom plot).

Next let us examine what a form factor for ${ }^{3} \mathrm{He}$, Figure 4.8 , and a form factor for ${ }^{3} \mathrm{H}$, Figure 4.20 , look like. They each have the oscillatory behavior predicted by the hard sphere of charge model indicating that their charge densities can be modelled similarly (in reality their charge densities decrease gradually with distance and not all at once). It is also interesting to study the form factors of individual nucleons like the proton. One might expect to find minima in the proton's form factors as well, however no such minima are observed. This is because the proton's form factors have an approximately dipole form, and the Fourier transform of a dipole is an exponential. This explains why we do not observe minima in the proton's form factors. However, it must be noted that the proton's form factors cannot be found by taking the Fourier transform of the electric or magnetic charge densities since for the Fourier transform to be valid the recoil of the system must be small. Since the proton is relatively light 
the recoil is not negligible and the Fourier transform is not valid.

As [1] points out the location of the diffractive minima can be used to learn about the size of the target. For a hard sphere of charge the charge radius, $R$, is roughly given by Equation 1.12, where $\frac{q}{\hbar}$ is the location of the first minima. One can also study the charge radius by examining the behavior of the charge density as it approaches zero. This can be seen by expanding the form factor in $q$ from the first integral in Equation 1.10. Euler's formula, $e^{i x}=\cos (x)+i \sin (x)$, begins the expansion of the exponent, but we are still left with a troublesome $i \sin (x)$ term. This $i \sin (x)$ term can be eliminated by assuming that the wavelength of the electron, $\frac{\hbar}{q}$, is much larger than the charge radius, $R$, as in Equation 1.13 .

$$
\begin{gathered}
R \approx \frac{4.5 \hbar}{q} \\
R \ll \frac{\hbar}{q} \Longrightarrow \frac{R q}{\hbar} \ll 1
\end{gathered}
$$

Now $i \sin \left(\frac{R q}{\hbar}\right) \rightarrow 0$ and we can drop the $i \sin (x)$ term leaving only $e^{i x} \approx \cos (x)$. The Taylor expansion of $\cos (x)$ is given by Equation 1.14 .

$$
\cos (x)=1-\frac{x^{2}}{2 !}+\frac{x^{4}}{4 !}-\frac{x^{6}}{6 !}+\ldots
$$

Keeping only the first two terms of the $\cos (x)$ expansion we can now rewrite the form factor equation as in Equation 1.15. Here we have inserted $q \cdot r=|q||r| \cos (\omega)$ where $\omega$ is the angle between $q$ and $r$.

$$
F\left(q^{2}\right)=\int_{0}^{\infty} \int_{-1}^{1} \int_{0}^{2 \pi} \rho(r)\left(1-\frac{1}{2} \frac{|q||r| \cos (\omega)}{\hbar}\right) r^{2} d \phi d \cos (\omega) d r
$$

Integrating over $\phi$ and $\cos (\omega)$ we obtain Equation 1.16 . 1 . 


$$
F\left(q^{2}\right)=4 \pi \int_{0}^{\infty} \rho(r) r^{2} d r-4 \pi \frac{q^{2}}{6 \hbar^{2}} \int_{0}^{\infty} \rho(r) r^{4} d r
$$

If we require that $\rho(r)$ be normalized such that $4 \pi \int_{0}^{\infty} \rho(r) r^{2} d r=1$ then we can define the mean square charge radius as in Equation 1.17 .

$$
\left\langle r^{2}\right\rangle=4 \pi \int_{0}^{\infty} r^{2} \rho(r) r^{2} d r
$$

Now Equation 1.16 can be rewritten as Equation 1.18.

$$
F\left(q^{2}\right)=1-\frac{q^{2}}{6 \hbar^{2}}\left\langle r^{2}\right\rangle
$$

Taking the derivative of Equation 1.18 with respect to $q^{2}$ we can extract $\left\langle r^{2}\right\rangle$ as in Equation 1.19. So by measuring the form factor of a target at very low $q^{2}$ one can calculate the mean square radius by finding the slope of the form factor at $q^{2}=0[1]$.

$$
\left\langle r^{2}\right\rangle=-\left.6 \hbar^{2} \frac{d F\left(q^{2}\right)}{d q^{2}}\right|_{q^{2}=0}
$$

At this point we are still claiming that the recoil of the struck particle is negligible. Let us examine the truth of this assumption by comparing the electron energies needed to study nuclear structure to the mass of a typical target, ${ }^{3} \mathrm{He}$. First let us determine approximately what energy an electron needs to be able to probe a target's nuclear structure. The Planck-Einstein relation can guide us with this estimate, and is given in Equation 1.20, where $h$ is Planck's constant and $\nu$ is the electron's frequency.

$$
E=h \nu
$$

Also remember the relationship between frequency and wavelength given in Equation 1.21

$$
\nu=\frac{c}{\lambda}
$$


Combining Equations 1.20 and 1.21 gives us Equation 1.22 which allows us to estimate the required electron energies to study nuclear structure.

$$
E=\frac{h c}{\lambda}
$$

Now plug in some reasonable values along with the constants. Nuclear targets are generally atoms made up of protons and neutrons so to glean any information about their structure one must use electrons with a wavelength approximately the size of, or smaller than that of, a proton. The proton's radius is about $0.84 \mathrm{fm}$. Plugging this into the wavelength value in Equation 1.22 along with $h \approx 4.136 \times 10^{-15} \mathrm{eVs}$ and $c \approx 3 \times 10^{8} \mathrm{~m} / \mathrm{s}$ yields an electron energy of about $1.477 \mathrm{GeV} .{ }^{3} \mathrm{He}$ has a mass of about $2.81 \mathrm{GeV}$. Clearly the electron energies required to study nuclear structure are no longer negligible when compared to target nuclei like ${ }^{3} \mathrm{He}$. This now requires us to account for target recoil in our cross section calculation from before.

Taking the recoil of the target in to account one finds that the recoil factor is given by Equation 1.23 from Equation 1.1 .

$$
\frac{E^{\prime}}{E_{0}}=\frac{1}{1+\frac{2 E_{0}}{M} \sin ^{2}\left(\frac{\theta}{2}\right)}
$$

Adding the factor of $\frac{E^{\prime}}{E_{0}}$ to the Mott cross section gives Equation 1.24 .

$$
\left(\frac{d \sigma}{d \Omega}\right)_{\mathrm{Mott}}=\frac{4 Z^{2} \alpha^{2}(\hbar c)^{2} E^{\prime 3}}{|q c|^{4} E_{0}} \cos ^{2}\left(\frac{\theta}{2}\right)
$$

The Mott cross section can be written in a slightly different form by setting some constants equal to one and rearranging some of the energies as in Equation 1.25. We now have an equation that represents the scattering of electrons off of a pointlike particle [1].

$$
\left(\frac{d \sigma}{d \Omega}\right)_{\mathrm{Mott}}=Z^{2} \frac{E^{\prime}}{E_{0}} \frac{\alpha^{2} \cos ^{2}\left(\frac{\theta}{2}\right)}{4 E_{0}^{2} \sin ^{4}\left(\frac{\theta}{2}\right)}
$$

At this point in the analysis we have accounted for charge, spin, relativity, and 
recoil. However, we have still neglected the fact that many targets have a magnetic moment, $\mu$, that will also interact with the electrons it scatters. Equation 1.26 shows the magnetic moment for a pointlike spin $1 / 2$ particle of mass $M$ where the $g=2$ factor comes from Dirac theory.

$$
\mu=g \frac{e \hbar}{4 M}
$$

To account for magnetic interactions we do as we did in Equation 1.7 and introduce a second term to the cross section. This term is scaled by a constant and given an angular dependence of $\sin ^{2}(\theta / 2)$ as in Equation 1.27. The angular dependence arises from a need to conserve angular momentum and helicity. We obtain $\tan ^{2}(\theta / 2)$ by pulling out a $1 / \cos ^{2}(\theta / 2)$.

$$
\left(\frac{d \sigma}{d \Omega}\right)_{\substack{\text { point } \\ \text { spin } 1 / 2}}=\left(\frac{d \sigma}{d \Omega}\right)_{\text {Mott }}\left(\left(1-2 \tau \tan ^{2}\left(\frac{\theta}{2}\right)\right)\right.
$$

$\tau$ is given by Equation 1.28 where $M$ is the target's mass and $Q^{2}$ is the kinematic variable measuring momentum transfer from Equation 1.3. From Equation 1.27 it becomes clear that at large angles and large momentum transfers the magnetic interaction becomes significant and cannot be ignored. The effect of the magnetic term causes the cross section to fall off less rapidly than it would if only the electric interaction were relevant [1].

$$
\tau=\frac{Q^{2}}{4 M}
$$

Finally let us consider that nuclear targets are not pointlike particles and thus the $g$-factor from Dirac is not precisely equal to two. Instead the $g$-factor is replaced by the proton and neutron magnetic moments given in Equation 1.29 quantified in terms of the nuclear magneton, $\mu_{N}$ given in Equation 1.30 , where $m_{p}$ is the proton mass.

$$
\mu_{p}=2.79 \mu_{N} \quad \mu_{n}=-1.91 \mu_{N}
$$




$$
\mu_{N}=\frac{e \hbar}{2 m_{p}}=3.1525 \times 10^{-8} e V T^{-1}
$$

We once again need to introduce form factors to describe the structure of the electric and magnetic components of the cross section. In this case we use the Sach's form factors $G_{E}\left(Q^{2}\right)$ and $G_{M}\left(Q^{2}\right)$ for the electric and magnetic components respectively. With these form factors we finally arrive at the Rosenbluth Equation 1.31 1.

$$
\left(\frac{d \sigma}{d \Omega}\right)=\left(\frac{d \sigma}{d \Omega}\right)_{M o t t}\left[\frac{G_{E}^{2}\left(Q^{2}\right)+\tau G_{M}^{2}\left(Q^{2}\right)}{1+\tau}+2 \tau G_{M}^{2}\left(Q^{2}\right) \tan ^{2}\left(\frac{\theta}{2}\right)\right]
$$

The physical meaning of these form factors can be further explored by studying their behavior as $Q^{2} \rightarrow 0 . G_{E}\left(Q^{2}\right)$ describes the electric structure of the target and therefore equals the electric charge of the target at $Q^{2}=0$ in units of elementary charge. $G_{M}\left(Q^{2}\right)$ describes the magnetic structure of the target and therefore equals the magnetic moment of the target at $Q^{2}=0$ in units of the nuclear magneton. So we find that at $Q^{2}=0$ the proton's form factors are given by Equation 1.32 and the neutron's form factors are given by Equation 1.33 .

$$
\begin{array}{cc}
G_{E}^{p}\left(Q^{2}=0\right)=1 & G_{M}^{p}\left(Q^{2}=0\right)=2.79 \\
G_{E}^{n}\left(Q^{2}=0\right)=0 & G_{M}^{n}\left(Q^{2}=0\right)=-1.91
\end{array}
$$

In the literature one finds several other commonly used form factors related to $G_{E}$ and $G_{M}$. Being able to translate between these form factors is often necessary to compare the results reported by different groups. The first set of these form factors are the Dirac form factor, $F_{1}$, and the Pauli form factor, $F_{2}$. These two form factors are given by Equations 1.34 and 1.35 in relation to $G_{E}$ and $G_{M}$ which are more commonly used because of their physical interpretation. 


$$
\begin{aligned}
& G_{E}\left(Q^{2}\right)=F_{1}\left(Q^{2}\right)-\mu \tau F_{2}\left(Q^{2}\right) \\
& G_{M}\left(Q^{2}\right)=F_{1}\left(Q^{2}\right)+\mu F_{2}\left(Q^{2}\right)
\end{aligned}
$$

One final set of form factors are $F_{c h}$ and $F_{m}$. Their relations to $G_{E}$ and $G_{M}$ are given in Equations 1.36 and 1.37 [2]. These are the form factors used in the sum of Gaussians analysis of the data presented in this thesis. To see what the ${ }^{3} \mathrm{He}$ and ${ }^{3} \mathrm{H} F_{c h}$ and $F_{m}$ form factors look like along with some world data see Figures 4.9 and 4.21 respectively.

$$
\begin{aligned}
& F_{c h}\left(Q^{2}\right)=G_{E}\left(Q^{2}\right) \\
& F_{m}\left(Q^{2}\right)=\frac{G_{M}\left(Q^{2}\right)}{\mu}
\end{aligned}
$$

The form factors $G_{E}$ and $G_{M}$ can be separated out from experimental data according to the procedure of Rosenbluth separation laid out in [2. First numerous cross section measurements at a fixed $Q^{2}$ and multiple angles must be taken. To see how to extract $G_{E}$ and $G_{M}$ it is helpful to rewrite the Rosenbluth equation in Equation 1.31 as Equation 1.38 with $\epsilon$ given in Equation 1.39. As an example of what a typical cross section looks like see Figure 4.13 which shows the ${ }^{3} \mathrm{He}$ cross section derived in this thesis.

$$
\begin{aligned}
\left(\frac{d \sigma}{d \Omega}\right)_{\exp } & =\left(\frac{d \sigma}{d \Omega}\right)_{M o t t} \frac{1}{1+\tau}\left[G_{E}^{2}\left(Q^{2}\right)+\frac{\tau}{\epsilon} G_{M}^{2}\left(Q^{2}\right)\right] \\
\epsilon & =\left(1+2(1+\tau) \tan ^{2}\left(\frac{\theta}{2}\right)\right)^{-1}
\end{aligned}
$$

Now we can define a value called the reduced cross section by dividing the experimental cross section by the Mott cross section and rearranging some kinematic factors as in Equation 1.40 . 


$$
\left(\frac{d \sigma}{d \Omega}\right)_{r}=\frac{\left(\frac{d \sigma}{d \Omega}\right)_{\text {exp }}}{\left(\frac{d \sigma}{d \Omega}\right)_{M o t t}} \epsilon(1+\tau)=\left[\epsilon G_{E}^{2}\left(Q^{2}\right)+\tau G_{M}^{2}\left(Q^{2}\right)\right]
$$

If one plots the reduced cross section against $\epsilon$ we see that we are plotting the equation of a line. Figure 1.3 shows an example of a Rosenbluth separation using data from $[3$. The first point used was taken at $3.304 \mathrm{GeV}$ and $27.24^{\circ}$ with a $\frac{d \sigma}{d \Omega}$ of $2.77 \pm 0.39 \times$ $10^{-13} \mathrm{~cm}^{2} / \mathrm{sr}$ and the second point was at taken at $0.9893 \mathrm{GeV}$ and $140.31^{\circ}$ with a $\frac{d \sigma}{d \Omega}$ of $3.27 \pm 0.13 \times 10^{-15} \mathrm{~cm}^{2} / \mathrm{sr}$. Both points have the same $Q^{2}$ value, $55.1 \mathrm{fm}^{-2}$, as required to perform a Rosenbluth separation.

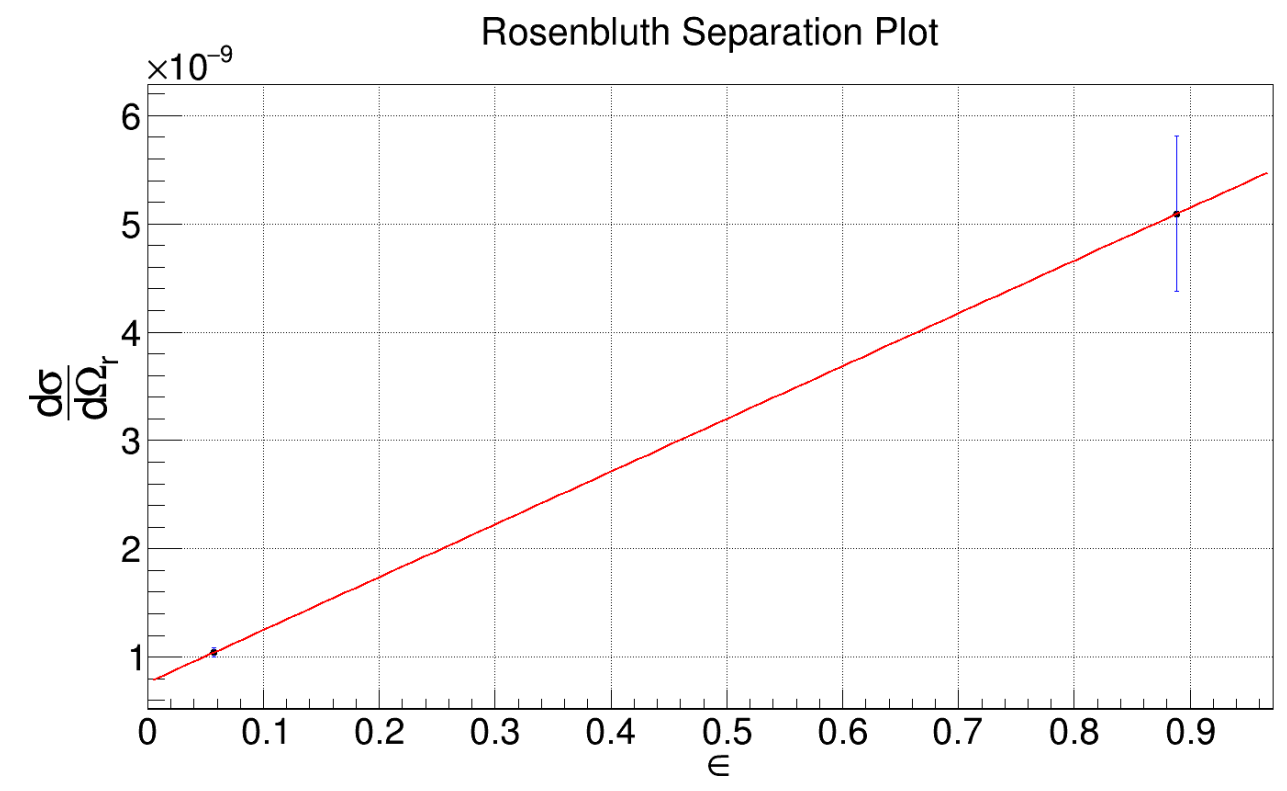

Figure 1.3: Rosenbluth Separtation. An example Rosenbluth separation using data at $Q^{2}=55.1 \mathrm{fm}^{-2}$ from 3 . From this separation we find $F_{c h}=6.98 \times 10^{-5}$ and $F_{m}=3.33 \times 10^{-5}$, where we have converted $G_{E}$ and $G_{M}$ to the $F_{c h}$ and $F_{m}$ form factors used in this analysis.

Immediately we can identify the slope of the line as $G_{E}^{2}\left(Q^{2}\right)$ and the $y$-intercept as $\tau G_{M}^{2}\left(Q^{2}\right)$ 1]. Taking the data from our example separation and converting $G_{E}$ and $G_{M}$ to $F_{c h}$ and $F_{m}$ we find that $F_{c h}=6.98 \times 10^{-5}$ and $F_{m}=3.33 \times 10^{-5}$, where we have neglected to treat the uncertainty for simplicity. These form factor values are in agreement with the calculations made in [3]. This method works well to extract the Sach's form factors if there are enough data points taken and they 
have sufficiently small error bars. Unfortunately, this method is very time consuming from an experimental standpoint. In Section 4.2 we will examine a different method of extracting form factors by fitting experimental cross section data using a sum of Gaussians technique.

\subsection{Motivation and Mirror Nuclei}

Why are we interested in finding the form factors of ${ }^{3} \mathrm{H}$ and ${ }^{3} \mathrm{He}$ ? Knowing these form factors allows us to calculate numerous useful quantities including the cross section at different energies, the charge radii, and the charge densities. The form factors also teach us about the three-body physics inside of the two nuclei, and thus they provide information on the total wave function. This information can then be compared with theoretical predictions, e.g. comparing the fitted form factors to theory predictions as in Sections 4.3.6 and 4.3.7, and then be used to improve the underlying theoretical models. If one wishes to study a 'free' neutron one often uses ${ }^{3} \mathrm{He}$ as a proxy system. These theoretical models are vital for correcting for three-body effects inside the nucleus so that a 'free' neutron can be studied, and all of these corrections are dependent on a firm understanding of the ${ }^{3} \mathrm{He}$ form factors.

This analysis has chosen to analyze both ${ }^{3} \mathrm{H}$ and ${ }^{3} \mathrm{He}$ as they are mirror nuclei. This means that each nuclei has the same number of nucleons, but the number of protons and neutrons in each nucleus is flipped (i.e. ${ }^{3} \mathrm{H}$ has one proton and two neutrons and ${ }^{3} \mathrm{He}$ has two protons and one neutron). The differences in the form factors of these mirror nuclei then teach us about the differences of the protons and the neutrons in the system by simply replacing one with the other. If the two nuclei are in the ground state then each has three nucleons in the $1 \mathrm{~S}_{1 / 2}$ shells. ${ }^{3} \mathrm{H}$ should have one proton in the $1 \mathrm{~S}_{1 / 2}$ shell and two neutrons in the $1 \mathrm{~S}_{1 / 2}$ shell for a full shell for the neutrons (so the angular momentum of ${ }^{3} \mathrm{H}$ is derived from the unpaired proton). ${ }^{3} \mathrm{He}$ should have two protons in the $1 \mathrm{~S}_{1 / 2}$ shell for a full shell and one neutron in the $1 \mathrm{~S}_{1 / 2}$ shell (so the angular momentum of ${ }^{3} \mathrm{He}$ is derived from the unpaired neutron). It is believed 
that the strong force treats both protons and neutrons identically. Therefore, changing between these nuclei does not change the ground state shell-model state but merely fills a different shell. Any differences in the form factors for these mirror nuclei are then due to the different Coulomb forces in the nuclei from the electric charges of the protons and neutrons [4].

Let us predict which nucleus, ${ }^{3} \mathrm{H}$ or ${ }^{3} \mathrm{He}$, has a larger charge radius and then study how that influences the form factors. To do this we will employ the liquid drop model of the nucleus. In this model the binding energy for a nucleus is given by Equation 1.41 [5]. The terms from left to right are the volume, surface, Coulomb, asymmetry, and pairing terms, and they are derived from how the nucleon's configurations and ratios contribute to the binding energy. In Equation $1.41 a_{1-4}$ are empirically derived constants, $A$ is the total number of nucleons, $Z$ is the number of protons, and $N$ is the number of neutrons.

$$
B(A, Z)=a_{1} A-a_{2} A^{2 / 3}-a_{3} \frac{Z(Z-1)}{A^{1 / 3}}-a_{4} \frac{(N-Z)^{2}}{A}+\delta
$$

Let us now apply this binding energy formula to our mirror nuclei. We see that the volume and surface terms are equal for ${ }^{3} \mathrm{H}$ and ${ }^{3} \mathrm{He}$ since both nuclei have $A=3$. The asymmetry terms are also equal due to squaring the numerator. The $\delta$ (pairing) term is zero for nuclei with odd numbers of nucleons. This leaves only the Coulomb term, $a_{3} \frac{Z(Z-1)}{A^{1 / 3}}$, to differentiate the binding energies as we anticipated since the strong force does not distinguish between protons and neutrons. For ${ }^{3} \mathrm{H}$ this term equals zero, and for ${ }^{3} \mathrm{He}$ this term equals $a_{3} \frac{2}{3^{1 / 3}}$. $a_{3}$ is experimentally determined to equal 0.645 $\mathrm{MeV}$ making the binding energy of ${ }^{3} \mathrm{He} 0.894 \mathrm{MeV}$ lower than that of ${ }^{3} \mathrm{H}[5]$. (The total binding energies of both nuclei are on the order of $8 \mathrm{MeV}$.) Ultimately, we find that the binding energies of these mirror nuclei are similar, but the nucleons in ${ }^{3} \mathrm{He}$ are less tightly bound.

Let us examine if this prediction agrees with our expectations. ${ }^{3} \mathrm{He}$ contains two protons and a neutron, whereas ${ }^{3} \mathrm{H}$ contains one proton and two neutrons. The nuclei 
of these atoms are held together by the strong force, while the Coulomb force pushes against this binding force. ${ }^{3} \mathrm{He}$ has more charge due to having two protons, and thus the Coulomb force for ${ }^{3} \mathrm{He}$ will be stronger than that of ${ }^{3} \mathrm{H}$. Therefore, we expect ${ }^{3} \mathrm{He}$ to have a larger charge radius than ${ }^{3} \mathrm{H}$ as there is a larger Coulomb force pushing the charge radius out against the strong force.

As 1] points out the location of the form factor's diffractive minima can be used to learn about the size of the charge radius (see Equation 1.12). Let us use this relation to compare the locations of the first minima in $F_{c h}$ for ${ }^{3} \mathrm{He}$ (Figure 4.8a) and ${ }^{3} \mathrm{H}$ (Figure 4.20a). ( $F_{c h}$ is discussed in more detail in Section 1.3, but it represents the Fourier transform of the electric charge density.) We see that the first minimum for ${ }^{3} \mathrm{He}$ is found at $Q^{2} \approx 11 \mathrm{fm}^{-2}$, and the first minimum for ${ }^{3} \mathrm{H}$ is found at $Q^{2} \approx 13$ $\mathrm{fm}^{-2}$. Equation 1.12 then predicts that ${ }^{3} \mathrm{He}$ has a slightly larger charge radius than ${ }^{3} \mathrm{H}$ exactly as we predicted from the liquid drop model. The comparisons between these two mirror nuclei are revisited in Section 4.3.8 using the new fits from this analysis. 


\section{Chapter 2}

\section{Experimental Setup}

\subsection{Overview}

The Thomas Jefferson National Accelerator Facility (Jefferson Lab or JLab) located in Newport News, Virginia uses the Continuous Electron Beam Accelerator Facility (CEBAF) to perform electron scattering experiments to study nuclear structure. Jefferson lab consists of four experimental halls designated Halls A, B, C, and the newly commissioned Hall D as shown in Figure 2.1. The facility is capable of creating electron beams of energies as high as $12 \mathrm{GeV}$ and supplying those electrons to the four halls simultaneously (Hall A is limited to $11 \mathrm{GeV}$ ). The $12 \mathrm{GeV}$ capability is a recently completed upgrade. At the time of the experiment discussed in this thesis, experiment E08-014, the facility was limited to $6 \mathrm{GeV}$ beam energy.

\subsection{Experiment E08-014}

Experiment E08-014 ran in Jefferson Lab's Hall A in 2011. The experiment used electron scattering to measure the inclusive cross sections, $\mathrm{N}\left(\mathrm{e}, \mathrm{e}^{\prime}\right)$, of various targets using both of Hall A's high resolution spectrometers (HRSs). E08-014 compared heavy targets to two and three-nucleon targets to study the short range correlations (SRC). To this end, inclusive cross sections for ${ }^{2} \mathrm{H},{ }^{3} \mathrm{He},{ }^{4} \mathrm{He},{ }^{12} \mathrm{C},{ }^{40} \mathrm{Ca}$, and ${ }^{48} \mathrm{Ca}$ were 


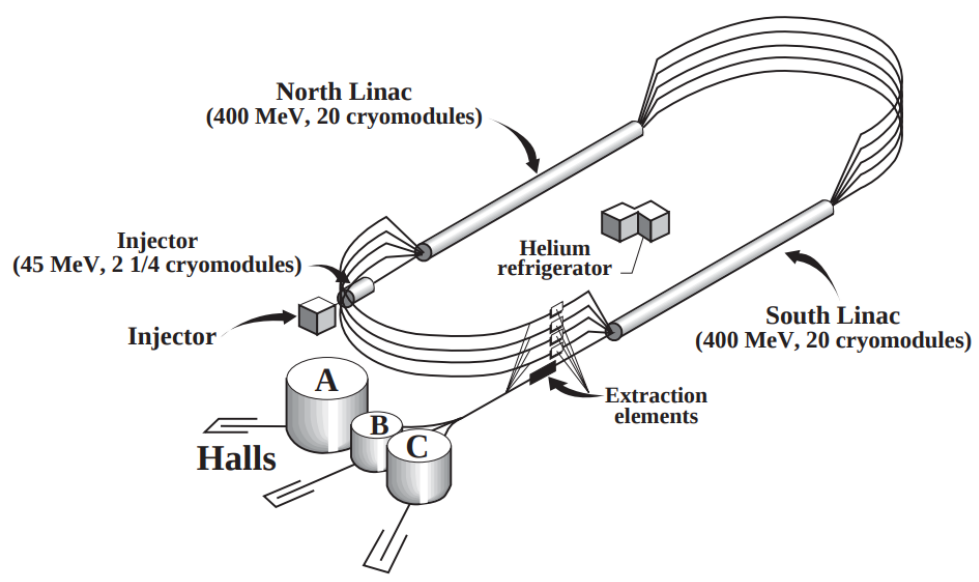

Figure 2.1: Thomas Jefferson National Accelerator Facility. CEBAF is the ring connecting to the experimental halls. This image is of the previous $6 \mathrm{GeV}$ configuration. Now Hall D is now located at the top right of the image. Image from 6.

measured in the region of $1.1 \mathrm{GeV}^{2}<Q^{2}<2.5 \mathrm{GeV}^{2}$. This experiment studied the region of $\left.1.3<x_{B j}<3[7] 8\right]$.

While experiment E08-014 focused on the QE region of electron scattering, one kinematic region, Kin 3.2 in Figure 2.2, also included elastically scattered electrons. The elastic events can be seen by plotting the scattering angle of the electron versus the scattered electron's energy, $E^{\prime}$, as shown in Figure 2.3. The resulting curve (red) gives the elastic scattering band for ${ }^{3} \mathrm{He}$. When this band is compared with the spectrometer's upper and lower acceptance in energy and angle, represented by the black lines, it becomes apparent that the elastic band passes through Kin 3.2. Thus we expect to find ${ }^{3} \mathrm{He}$ elastic data in Kin 3.2.

Now that we believe there should be elastically scattered electrons in our data let us see if we can detect them. To find these electrons we will search for the elastic peak in $x_{B j}$ that we discussed in Section 1.1. Figure 2.4 shows a plot of $x_{B j}$ for the summed production runs of Kin 3.2. The large initial distribution is the quasielastic peak of ${ }^{3}$ He. Looking at $x_{B j}=3$ it is clear that there is another smaller peak indicating the presence of elastically scattered electrons. The smaller peak contains around a maximum of 1000 electrons, which while enough for a cross section extraction, places 


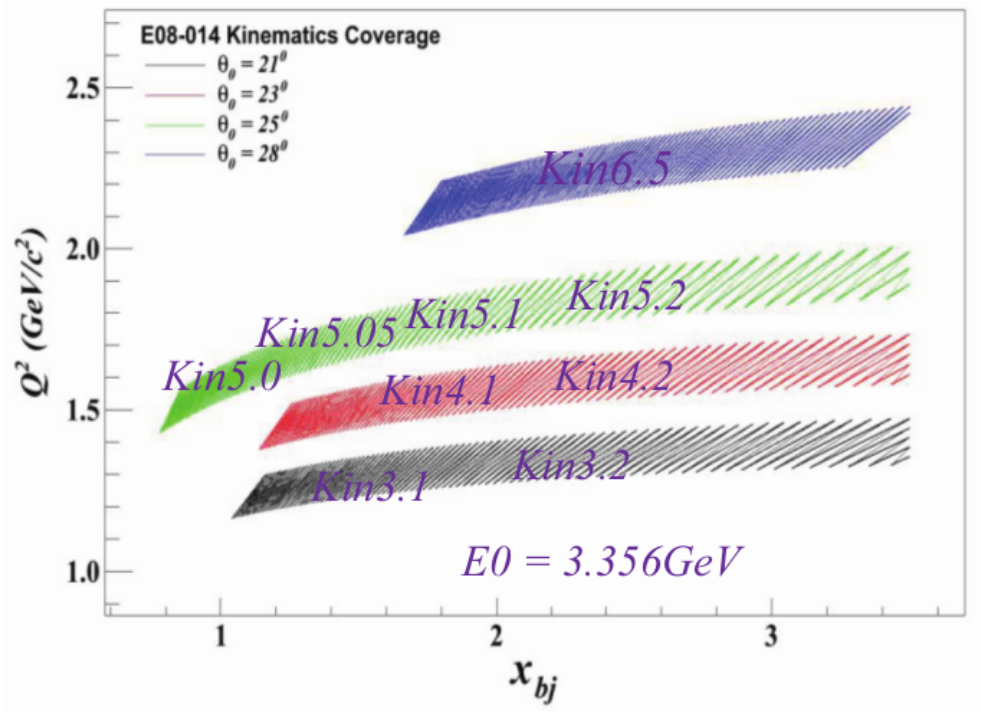

Figure 2.2: Kinematic Coverage of Experiment E08-014. Kinematic coverage of experiment E08-014. The elastic ${ }^{3} \mathrm{He}$ data used in this analysis is located in Kin 3.2. Image from [7].

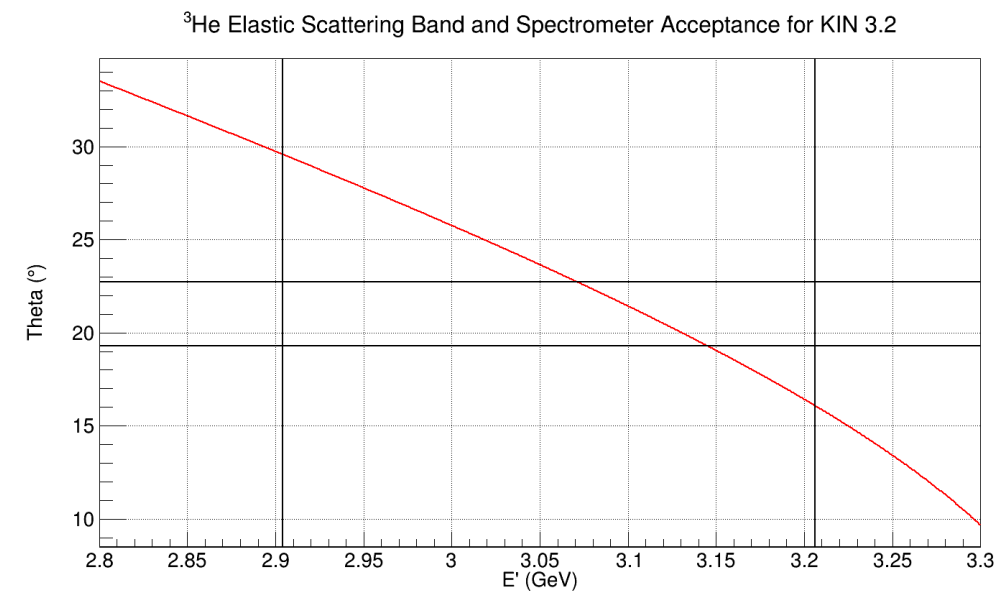

Figure 2.3: Elastic Band for ${ }^{3}$ He. The box made by the intersecting black lines represents the maximum and minimum spectrometer acceptances in energy and angle for Kin 3.2. The red line is the elastic scattering band. Clearly the red elastic band passes through the spectrometer's acceptance so we expect to find elastic events in Kin 3.2 .

a limit on the uncertainty of our measurement. This electron yield will also decrease as we begin to impose cuts on the data.

These elastic events were scattered from a gaseous ${ }^{3} \mathrm{He}$ target and used to extract 


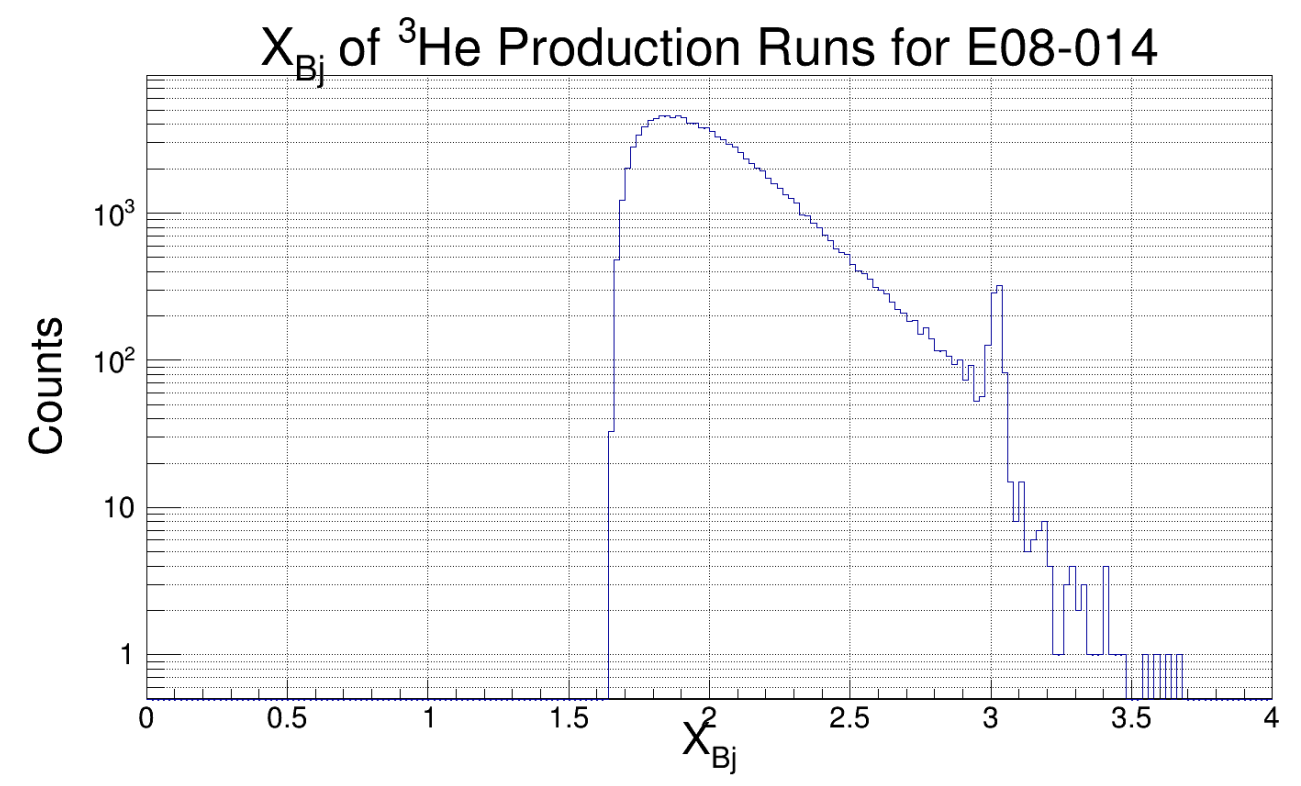

Figure 2.4: Elastic Peak in $x_{B j}$. The elastic peak is located at approximately $x_{B j}=3$ as expected. Notice that there is a large quasielastic background in this electron sample in addition to the elastic electrons in the elastic peak.

an elastic ${ }^{3} \mathrm{He}$ cross section. This new measurement is located in the little studied region of $Q^{2}=35 \mathrm{fm}^{-2}$ as seen in Figure 2.5 which shows the ${ }^{3} \mathrm{He}$ charge form factor, $F_{c h}$. This understudied region is interesting because it has the potential to constrain and improve previous fits of the ${ }^{3} \mathrm{He}$ form factors. In particular, high $Q^{2}$ data points like this help to pin down the magnetic form factor. Equation 1.31 makes clear that to measure the magnetic form factor's contribution to the cross section large $Q^{2}$ values and large back angles are required. Unfortunately, there are few measurements in the world data of high enough $Q^{2}$ to understand the magnetic form factor's behavior after its first minima.

\subsection{CEBAF}

Jefferson Lab's Continuous Electron Beam Accelerator Facility (CEBAF) uses superconducting radio frequency (SRF) cavities to accelerate electrons to energies up to $12 \mathrm{GeV}$ after a recent upgrade. However, this upgrade was completed after 


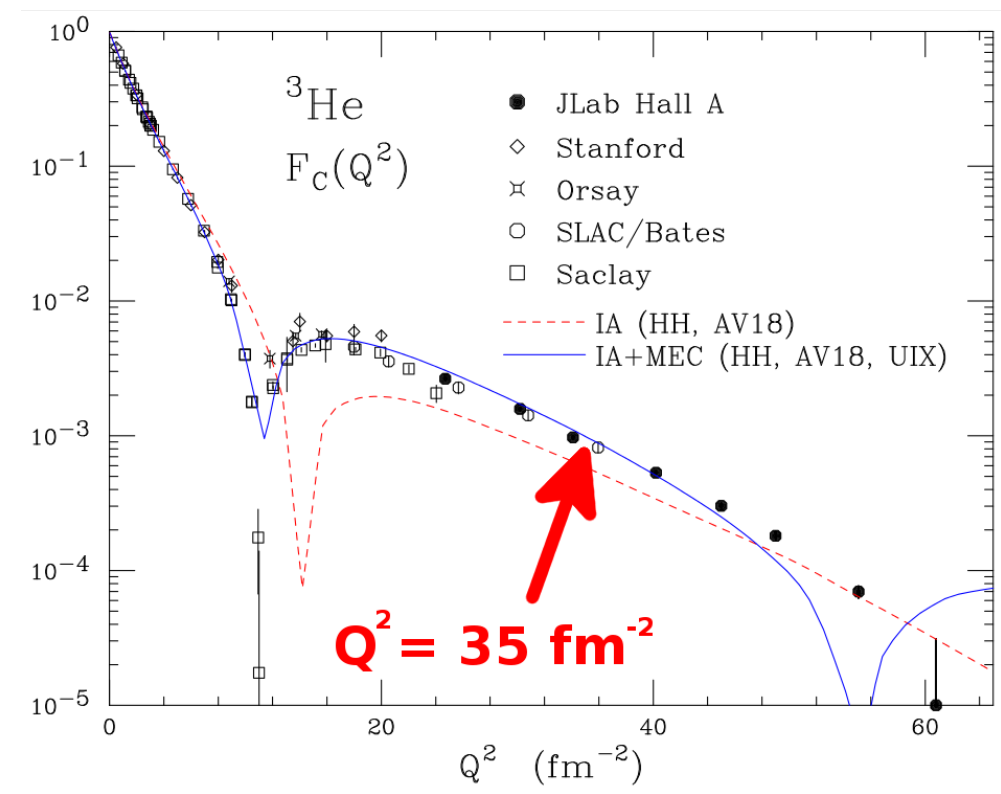

Figure 2.5: Location of New Elastic Measurement. This plot shows the $Q^{2}$ region of the ${ }^{3} \mathrm{He}$ electric form factor, $F_{c h}$, where the new elastic ${ }^{3} \mathrm{He}$ measurement is located. The $Y$-axis is $\left|F_{c h}\left(Q^{2}\right)\right|$. Image from 3 .

this experiment and as such this section will discuss the $6 \mathrm{GeV}$ era beam before the upgrade and Hall D was built. The accelerated electrons form polarizable continuous wave $(\mathrm{CW})$ beams that can be delivered to up to four scientific halls simultaneously for use in nuclear physics experiments. These beams have a maximum energy of 5.7 $\mathrm{GeV}$ and a maximum current of $200 \mu \mathrm{A}$. The accelerator can split this current among the three experimental halls [9].

CEBAF begins creating an electron beam using either a thermionic or polarized gun to inject electrons into the accelerator. The polarized gun produces electrons by illuminating a GaAs cathode crystal with a diode laser pulsed at $1497 \mathrm{MHz}$. (While CEBAF operates with a 'continuous wave' beam, if one looks at small enough time lengths the beam is still pulsed. This is due to needing to inject the electrons into the $\mathrm{RF}$ cavities at the proper time to have the standing waves in the RF cavities accelerate them.) These electrons then enter the first (North) of two linacs each of which contain 20 cryomodules that accelerate the electrons with a maximum gradient exceeding 7 $\mathrm{MeV} / \mathrm{m}$. At the end of the North linac the electrons are bent around a $180^{\circ}$ bend and 
enter the South linac passing through 20 more cryomodules. Upon reaching the end of the South linac the beam can be directed into any of the three halls by means of $\mathrm{RF}$ separators and septa. If higher energies are desired the beam can be recirculated through the linacs up to four additional times for a maximum of five passes through the accelerator resulting in the maximum energy of $5.7 \mathrm{GeV}[6]$.

\subsection{Hall A Beamline}

The distinguishing feature of Jefferson Lab's experimental Hall A are the two High Resolution Spectrometers (HRSs) and their associated detector packages. At a central momentum setting of $4 \mathrm{GeV}$ these two spectrometers provide a momentum resolution better than $\frac{\delta p}{p}=2 \times 10^{-4}$ as well as a horizontal angular resolution of more than 2 mrad. The spectrometer magnets bend the particles upward into the detector stack using a series of quadrupole and dipole magnets in a QQDQ arrangement 6]. A side view of Hall A is given in Figure 2.6 and a top view is given in 2.7. Each of the components listed in 2.7 will be discussed individually in the following sections.

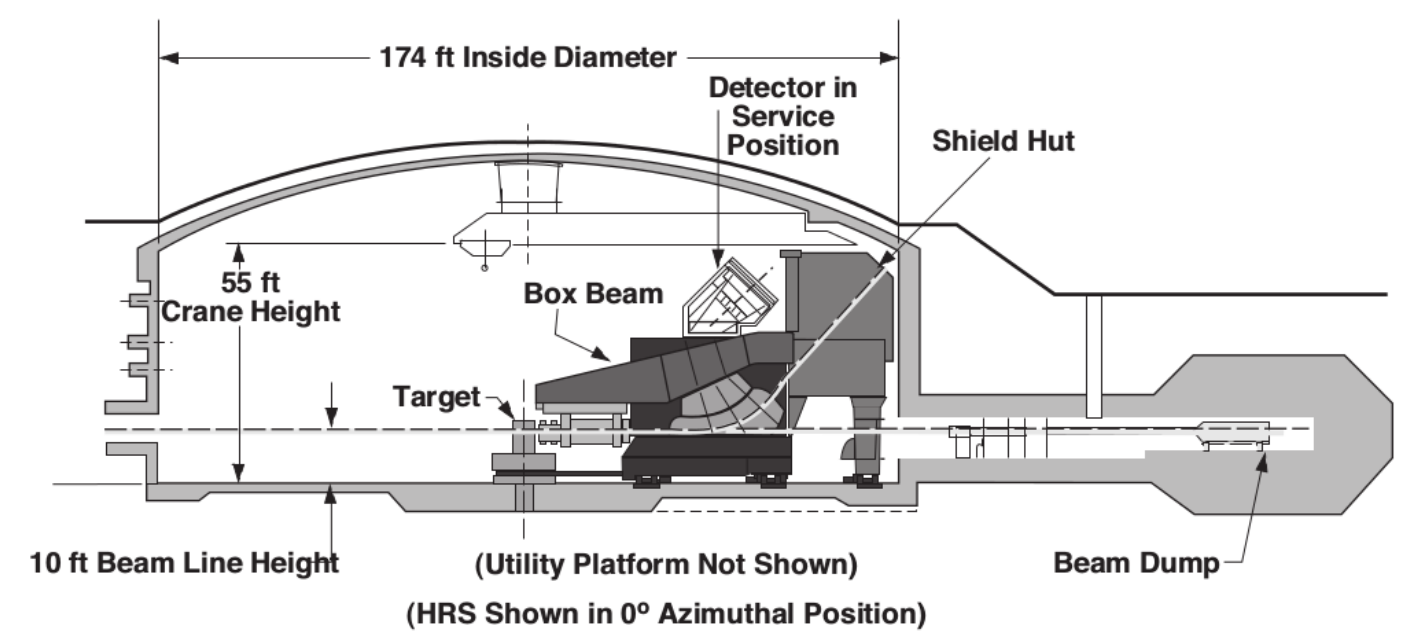

Figure 2.6: Hall A Side View. The electron beam enters the hall from the left side of the image. It then interacts with the target at the hall's center. The scattered particles then pass through the High Resolution Spectrometers which bend the particles upward $45^{\circ}$ where they enter the detector stacks. Image from [6]. 


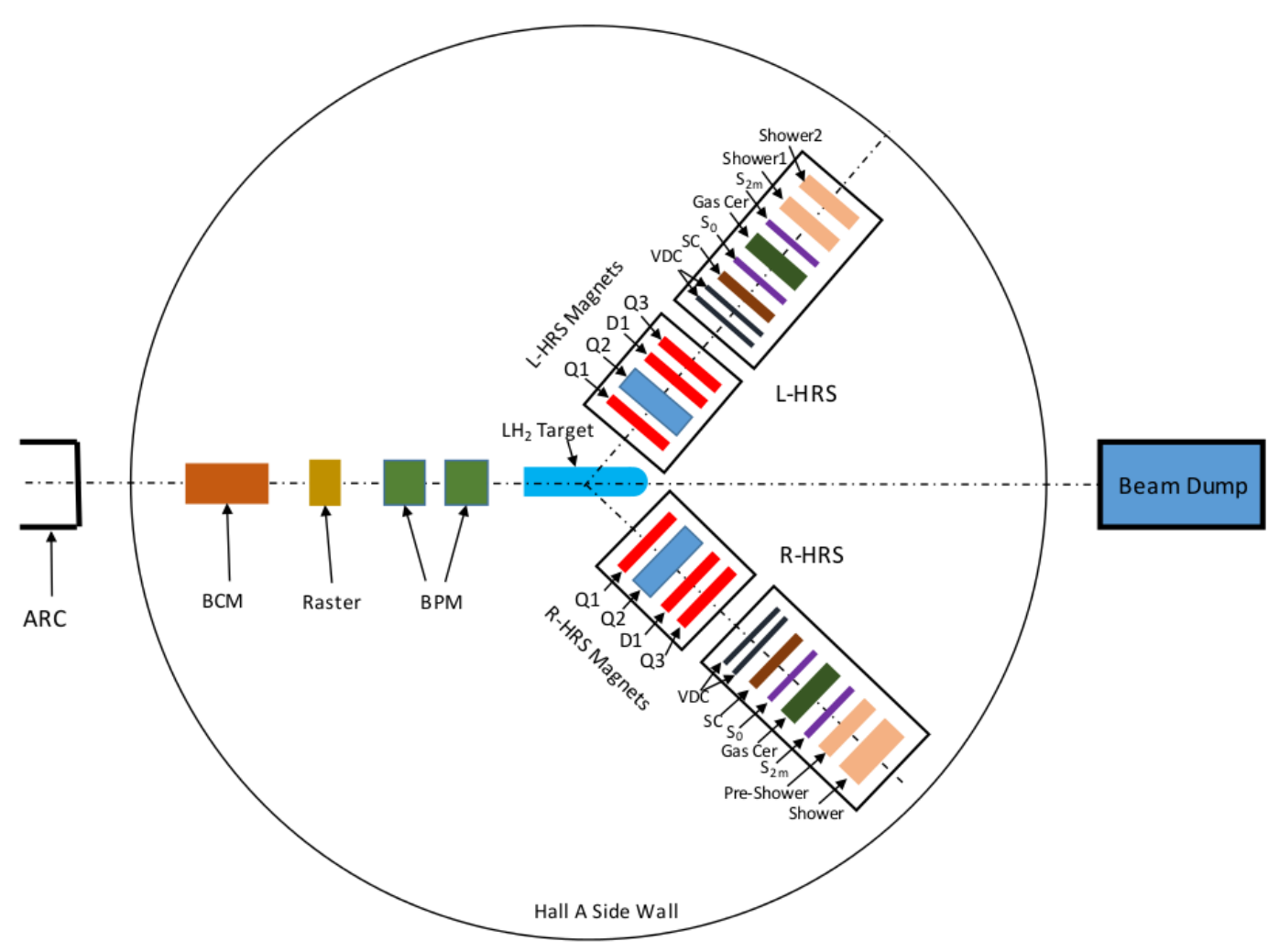

Figure 2.7: Hall A Top View. The electron beam enters the hall from the left side of the image. It then passes through the beam current monitors which measure the current. The beam then goes through a raster to avoid overheating one area of the target. Next the beam passes through the beam position monitors which measure its position. It then interacts with the target at the hall's center. The scattered particles then pass through the High Resolution Spectrometers which bend the particles upward $45^{\circ}$ where they enter the detector stacks. In the stacks the particles pass through the vertical drift chambers, used for trajectory reconstruction, followed by the straw chambers. They then pass through the first set of scintillator paddles, $\mathrm{S}_{0}$, followed by the gas Cherenkov, before passing through the second set of scintillator paddles, $\mathrm{S}_{2 m}$. A coincidence of $\mathrm{S}_{0}, \mathrm{~S}_{2 m}$, and the GC creates the main production trigger. The GC also performs particle identification. Finally the particles enter the electromagnetic shower calorimeters which help further aid particle identification. Image from 10.

\subsubsection{Beam Energy}

An accurate measure of the electron beam's energy is necessary to obtain accurate experimental results. The energy of the electron beam was measured using the Arc method laid out in [6. This method works by passing the electron beam through a series of dipole magnets in the arc section of the beam line and measuring its deflection 
as shown in Figure 2.8. The beam's momentum, $|\vec{p}|$, is then given by the field integral of the eight dipole magnets, $\int \overrightarrow{\mathbf{B}} \cdot \overrightarrow{\mathbf{d}}$, divided by the arc bend angle, $\phi_{\text {arc }}$, multiplied by a constant $C_{\text {arc }}=0.299792 \mathrm{GeV} \mathrm{rad} \mathrm{T}^{-1} \mathrm{~m}^{-1} / \mathrm{c}$ as given in Equation 2.1. To perform this calculation two measurements are required. The first measurement is of the magnetic field integral of the eight dipoles and is made based on a ninth reference dipole. The second measurement is of the bend angle of the arc which is measured by a set of wire scanners (Super Harps).

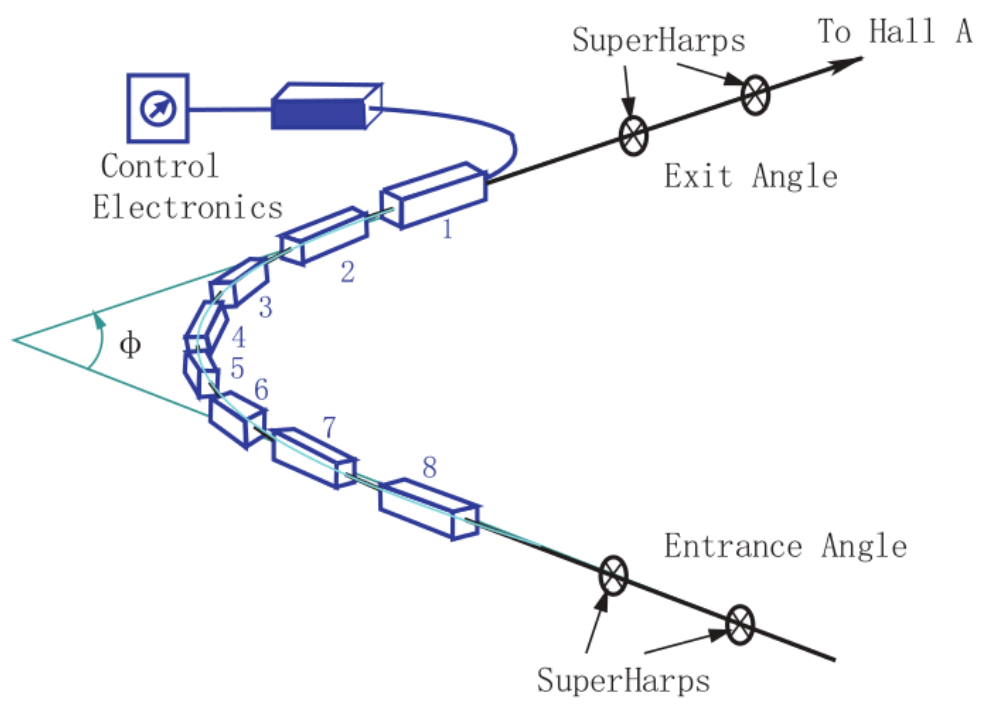

Figure 2.8: Arc Energy Measurement Diagram. The electron beam is bent through an angle $\phi_{\text {arc }}$ by a series of eight dipole magnets. Image from 10 .

$$
|\vec{p}|=C_{a r c} \frac{\int \overrightarrow{\mathbf{B}} \cdot \overrightarrow{\mathbf{d}} \mathbf{l}}{\phi_{a r c}}
$$

\subsubsection{Beam Position Monitors}

Along with the beam's energy its position must also be well known. To assess the beam's position two beam position monitors (BPMs) are located $7.524 \mathrm{~m}$ and 1.286 m upstream of the Hall A target [6]. Figure 2.9 shows the layout of the beamline components. Each of these BPMs is made up of four orthogonally oriented antennae placed perpendicular to the beam. For beam currents above $1 \mu \mathrm{A}$ these antennae 
produce a signal that is inversely proportional to the beam's distance from the antennae. The position of the beam is determined by the difference-over-sum technique laid out in [11] and [12].

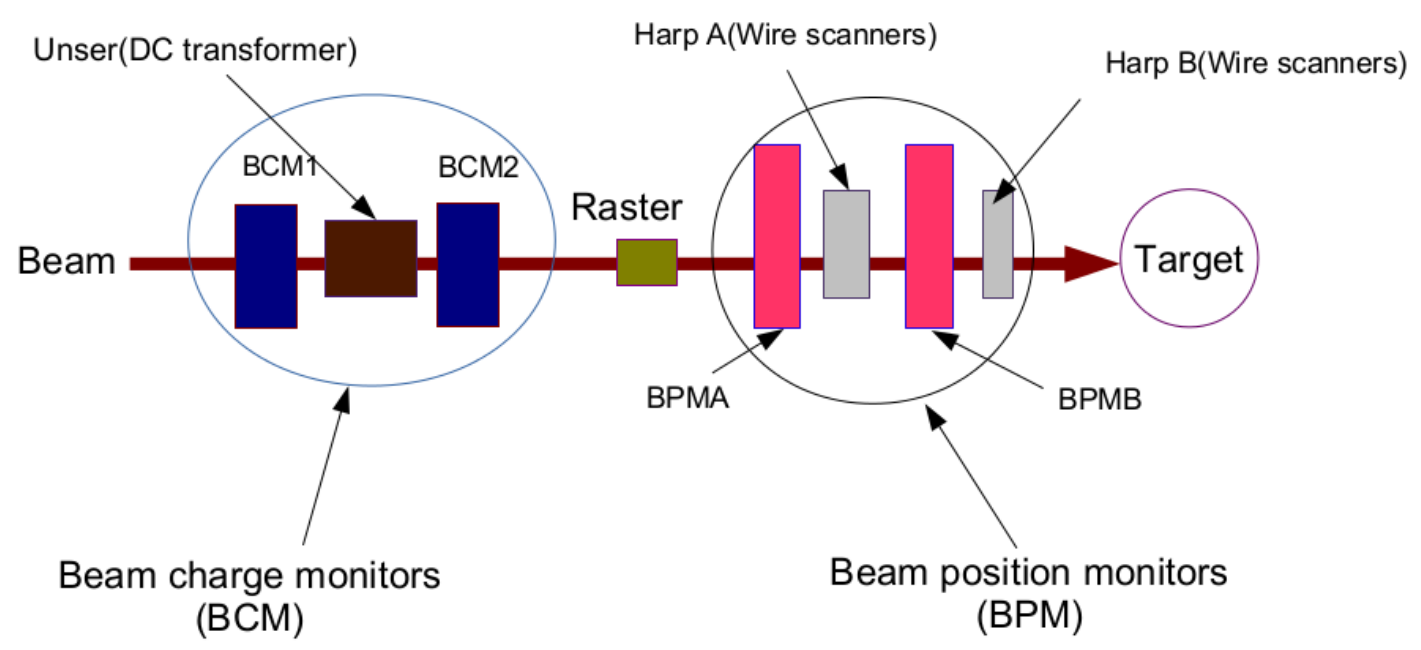

Figure 2.9: Layout of Hall A Beamline Components. Prior to striking the target the electron beam passes through the two beam current monitors, BCM1 and BCM2, and the Unser which measure the beam current. The beam then passes through the raster which uses steering magnets to spread the beam out over the surface of the target. Finally the beam passes through two beam position monitors, BPMA and BPMB, which measure the beam's position. Image from [10].

The BPMs are calibrated by wire scanners adjacent to each of them. These wire scanners are independently calibrated periodically with respect to the Hall A coordinates and are accurate to within $200 \mu \mathrm{m}$. The data from the BPMs is recorded in the EPICS database every second and in the data stream every three to four seconds. Each of the eight antennae signals is also recorded in the CODA data stream on an event by event basis [7.

\subsubsection{Raster}

The electron beam is generally quite narrow at $<0.3 \mathrm{~mm}[10]$. If this small beam falls on a single part of the target the target can overheat locally causing parts of the target to behave differently than those not struck by the beam. There is also the risk 
of burning a hole through the target. To avoid these problems the electron beam is rastered, meaning that the beam is spread out over a larger area of the target. The fast raster consists of two steering magnets and operates with a frequency of 17-24 $\mathrm{kHz}$. It is located $23 \mathrm{~m}$ upstream from the target [6] as seen in Figure 2.9. This system has the capability to spread the beam over several $\mathrm{mm}$ in all directions illuminating the target uniformly.

\subsubsection{Beam Current Monitors}

The beam current is measured by two beam current monitors (BCMs), which are RF cavity monitors, and an Unser located $25 \mathrm{~m}$ upstream of the target $[6$ as shown in Figure 2.9. The Unser measures beam current by, "a system combining a second harmonic magnetic modulator with an active current transformer in an operational feedback loop, to obtain wide band response down to de" [13]. The two BCM RF cavities are located on either side of the Unser and are tuned to the electron beam frequency of $1497 \mathrm{MHz}$ (see Section 2.3).

These RF cavity monitors then produce a signal that is proportional to the beam current which is recorded by a data acquisition system. Before being recorded the output signals of the two BCMs are each split into three. One of those three signals is amplified by a factor of three and another by a factor of ten. This results in six signals from the two BCMs designated $\mathrm{U}_{1}, \mathrm{U}_{3}, \mathrm{U}_{10}, \mathrm{D}_{1}, \mathrm{D}_{3}$, and $\mathrm{D}_{10}$. The procedure and results for the BCM calibration for experiment E08-014 can be found in 14.

\subsection{Target}

Hall A has a cryogenic distribution system (CDS) capable of cooling targets to temperatures of around $5 \mathrm{~K}$ to $15 \mathrm{~K}[6]$. The targets are kept in a vacuum scattering chamber where they are allowed to interact with the electron beam. Inside this chamber

is a ladder containing various experimental targets as shown in Figure 2.10. The target ladder is divided into three loops which are cryogenically cooled while having their 
pressure and temperature monitored continuously. During experiment E08-014 loops one and two contained one each of a $10 \mathrm{~cm}$ and $20 \mathrm{~cm}$ target cell while loop 3 contained two $20 \mathrm{~cm}$ target cells. The target ladder can be moved up and down to place different targets in the path of the electron beam from the counting house [7].

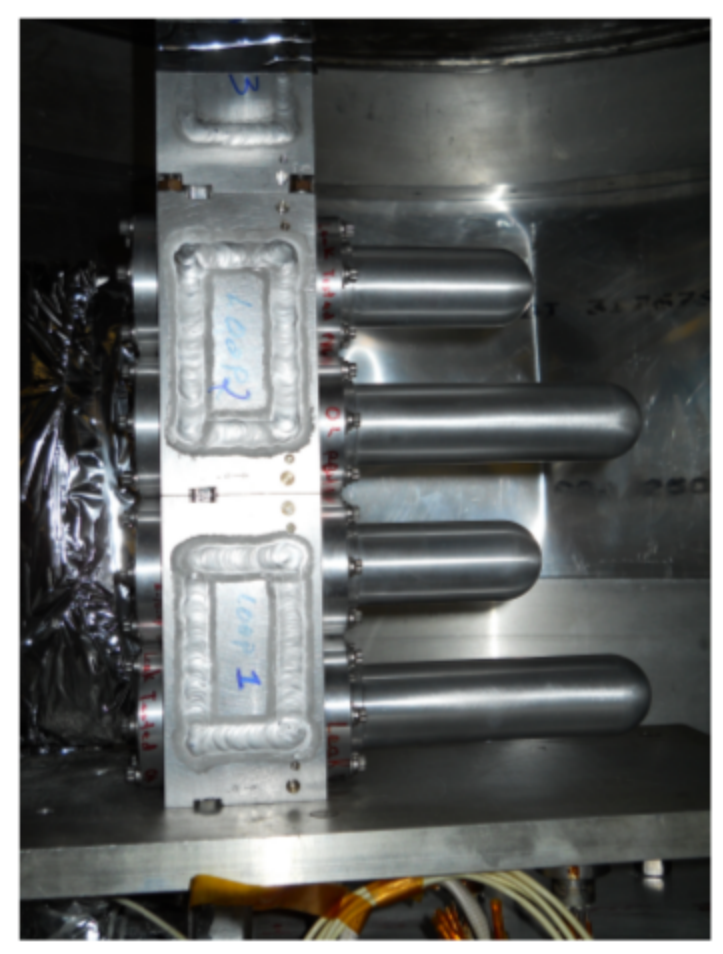

Figure 2.10: Target Ladder for Experiment E08-014. Coolant is flowed through the target ladder to maintain the targets' temperatures. The cylinders on the right of the image contain the target gasses. Only loops one and two are shown in this image. Image from [7].

Experiment E08-014 used a liquid deuterium target as well as a gaseous ${ }^{3} \mathrm{He}$ target, examined in this thesis, and a gaseous ${ }^{4} \mathrm{He}$ target. The ${ }^{3} \mathrm{He}$ target was used in the second run period of the experiment taking place from April $21^{\text {st }}$ to May $15^{\text {th }}$ of 2011. The ${ }^{3} \mathrm{He}$ target was located in loop 1 and was cooled to $17 \mathrm{~K}$ with a pressure of 211 psia [7]. The entire target layout over the two runs can be seen in Figure 2.11 and the monitoring and control system is shown in Figure 2.12 .

A $30 \mathrm{~cm}$ long carbon foil optics target was installed below loop 3. This target contains seven carbon foils spaced $5 \mathrm{~cm}$ apart ranging from $-15 \mathrm{~cm}$ to $+15 \mathrm{~cm}$. Electrons 


\begin{tabular}{lccccc}
\hline Target & $\rho\left(\mathrm{g} / \mathrm{cm}^{3}\right)$ & Length $(\mathrm{cm})$ & $\delta \rho\left(\mathrm{g} / \mathrm{cm}^{2}\right)$ & $\mathrm{I}(\mu \mathrm{A})$ & Comment \\
\hline $\mathrm{LD}_{2}$ & 0.1676 & 20.0 & $\mathrm{~N} / \mathrm{A}$ & 40 & Loop2 \\
$\mathrm{Al}$ can (Loop-2) & 2.7 & 0.0272 & 0.0001 & & Entrance \\
& 2.7 & 0.0361 & 0.0011 & & Exit \\
& 2.7 & 0.0328 & 0.0002 & Wall \\
${ }^{3} \mathrm{He}$ & 0.0296 & 20.0 & $\mathrm{~N} / \mathrm{A}$ & 120 & Loop1 \\
${ }^{4} \mathrm{He}$ & 0.0324 & 20.0 & $\mathrm{~N} / \mathrm{A}$ & 90 & Loop1 \\
$\mathrm{Al}-$ can (Loop-1) & 2.7 & 0.0272 & 0.0002 & & Entrance \\
& 2.7 & 0.0361 & 0.0006 & & Exit \\
& 2.7 & 0.0328 & 0.0005 & & Wall \\
${ }^{12} \mathrm{C}$ & 2.265 & 0.3937 & 0.0008 & 120 & \\
${ }^{40} \mathrm{C}$ & 1.55 & 0.5735 & 0.01 & 40 & Loop3 \\
${ }^{48} \mathrm{C}$ & 1.55 & 0.5284 & 0.01 & 40 & Loop3 \\
$\mathrm{Al}-$ can (Loop-3) & 2.7 & 0.0272 & 0.0001 & & Entrance \\
& 2.7 & 0.0361 & 0.001 & & Exit \\
& 2.7 & 0.0328 & 0.0002 & & Wall \\
Dummy-20cm & 2.7 & 0.1581 & 0.0005 & 40 & Upstream \\
& 2.7 & 0.1589 & 0.0005 & & Downstream \\
Dummy-10cm & 2.7 & 0.1019 & 0.0003 & 40 & Upstream \\
& 2.7 & 0.1000 & 0.0003 & & Downstream \\
\hline
\end{tabular}

Figure 2.11: Table of Target Information for Experiment E08-014. Image from [7.

scattering off of these foils can be used to calibrate the position of the target from data collected by the detector package as will be discussed in 2.10. Along with these targets a $10 \mathrm{~cm}$ and a $20 \mathrm{~cm}$ dummy target were also installed below the optics target. These dummy targets each contained two thick aluminium foils separated by $10 \mathrm{~cm}$ and 20 $\mathrm{cm}$ respectively. The dummy targets were used to study the end cap contributions of the target cells. There were also a $\mathrm{BeO}, \mathrm{a}^{12} \mathrm{C}$, and an empty target installed below 7 .

\subsection{High Resolution Spectrometers}

The high resolution spectrometers (HRSs) are the workhorses of Hall A. They are designated the left HRS (LHRS) and right HRS (RHRS) for the spectrometers on the left and right side of the beam direction respectively. While they were designed to be identical they each have unique features based on their construction as well as the general wear and tear of age on their components. They have a momentum resolution of $1 \times 10^{-4}$ from $0.8 \mathrm{GeV}$ to $4 \mathrm{GeV}$. They use a combination of superconducting quadrupole 


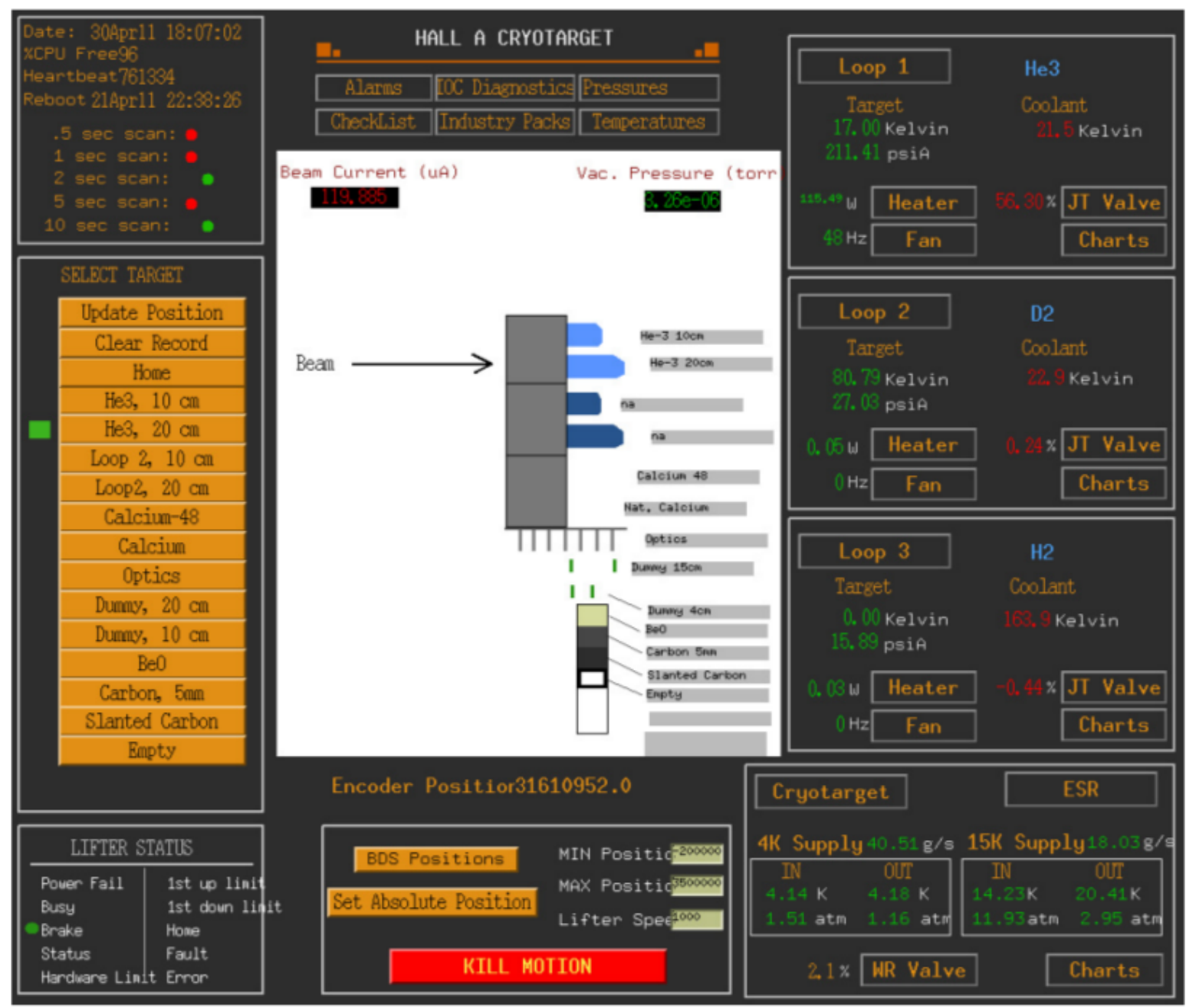

Figure 2.12: Cryogentic Monitoring and Control Screen. This control panel monitors and controls the temperatures of the individual targets. This panel is also used to move the target ladder up and down so that different targets can be placed in the electron beam. Image from $[7$.

and dipole magnets in a QQDQ combination to bend the scattered particles through a $45^{\circ}$ angle up into the detector stack as shown in Figure 2.13. The HRSs were designed to provide, "a large acceptance in both angle and momentum, good position and angular resolution in the scattering plane, an extended target acceptance, and a large angular range. [6]"

The superconducting quadrupoles are labelled Q1, Q2, and Q3 as shown in 2.13. The Q1 magnet provides focussing of the particles in the vertical plane, and the identical 


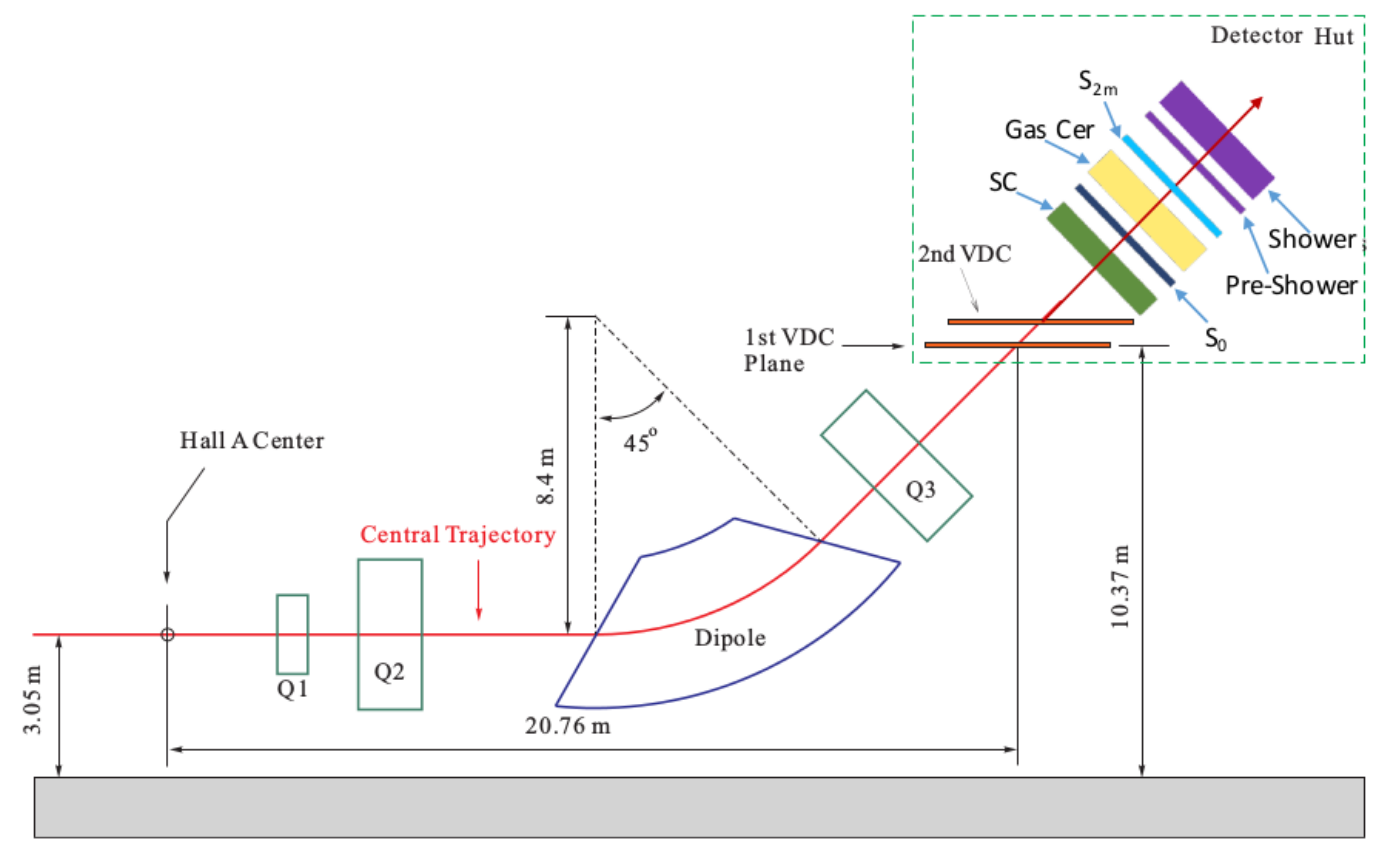

Figure 2.13: Side View of Single HRS. After interacting with the target the scattered particles pass through the High Resolution Spectrometers. These spectrometers have three quadrapole focusing magnets and a dipole magnet which bends the particles upward $45^{\circ}$ to the detector stacks. In the stacks the particles pass through the vertical drift chambers, used for trajectory reconstruction, followed by the straw chambers. They then pass through the first set of scintillator paddles, $\mathrm{S}_{0}$, followed by the gas Cherenkov, before passing through the second set of scintillator paddles, $\mathrm{S}_{2 m}$. A coincidence of $\mathrm{S}_{0}, \mathrm{~S}_{2 m}$, and the GC creates the main production trigger. The GC also performs particle identification. Finally the particles enter the electromagnetic shower calorimeters which help further aid particle identification. Image from [10].

Q2 and Q3 provide focussing in the transverse plane. The $6.6 \mathrm{~m}$ long superconducting dipole magnet bends the particles through a $45^{\circ}$ angle while providing additional focussing and the ability to use extended targets 6 . The primary characteristics of the HRSs are given in Figure 2.14. Unfortunately, during the run period the Q3 power supply of the RHRS was malfunctioning and could not reach the central momentum setting of $3.055 \mathrm{GeV}$ required for the experiment. As such this analysis considers only data from the LHRS [7]. 


\begin{tabular}{ll}
\hline Configuration & $\mathrm{QQD}_{n} \mathrm{Q}$ vertical bend \\
Bending angle & $45^{\circ}$ \\
Optical length & $23.4 \mathrm{~m}$ \\
Momentum range & $0.3-4.0 \mathrm{GeV} / c$ \\
Momentum acceptance & $-4.5 \%<\delta \mathrm{p} / \mathrm{p}<+4.5 \%$ \\
Momentum resolution & $1 \times 10^{-4}$ \\
Dispersion at the focus $(D)$ & $12.4 \mathrm{~m}$ \\
Radial linear magnification $(M)$ & -2.5 \\
$D / M$ & 5.0 \\
Angular range & $12.5-150^{\circ}$ \\
$\quad$ HRS-L & $12.5-130^{\circ}$ \\
$\quad$ HRS-R & \\
Angular acceptance & $\pm 30 \mathrm{mrad}$ \\
$\quad$ Horizontal & $\pm 60 \mathrm{mrad}$ \\
$\quad$ Vertical & \\
Angular resolution & $0.5 \mathrm{mrad}$ \\
$\quad$ Horizontal & $1.0 \mathrm{mrad}$ \\
$\quad$ Vertical & $6 \mathrm{msr}$ \\
Solid angle at $\delta \mathrm{p} / \mathrm{p}=0, y_{0}=0$ & $\pm 5 \mathrm{~cm}$ \\
Transverse length acceptance & $1 \mathrm{~mm}$ \\
Transverse position resolution & \\
\hline
\end{tabular}

Figure 2.14: Table of Important HRS Values. Image from 6.

\subsection{Detector Package}

The standard detector package for Hall A can be seen in Figures 2.7 and 2.13. These components include VDCs, scintillator planes, gas Cherenkovs, and electromagnetic calorimeters as well as the corresponding data acquisition systems. Each of these components will be described in the following sections.

\subsubsection{Vertical Drift Chambers}

After passing through the HRSs the scattered charged particles first pass through two vertical drift chambers (VDCs). These chambers each contain two planes of 368 wires each that are designated $\mathrm{U}_{1}$ and $\mathrm{V}_{1}$ for the bottom VDC and $\mathrm{U}_{2}$ and $\mathrm{V}_{2}$ for the top VDC. These pairs of planes are oriented at a $45^{\circ}$ angle to one another and are separated by $0.335 \mathrm{~m}$ as shown in Figure 2.15. The lower VDC lies in the spectrometer focal plane and the upper VDC allows angular reconstruction of particle trajectories [15].

The VDCs' interiors, Figure 2.16, were filled with a $62 \%-38 \%$ mixture of argon and 
ethane gasses flowed through at a rate of 10 liter/hour. Two gold-plated mylar planes sit above and below the sense wires and a large electric field is created between them on the order of $-4 \mathrm{kV}$. As charged particles pass through the gas the gas molecules become ionized and release electrons. The electric field then attracts these electrons to the sense wires which then pass the signal from those electrons through amplifier cards and on to the data acquisition system. The position of the particle can be resolved to around $100 \mu \mathrm{m}$ and the angle can be resolved to about $100 \mathrm{mrad}$ [7].
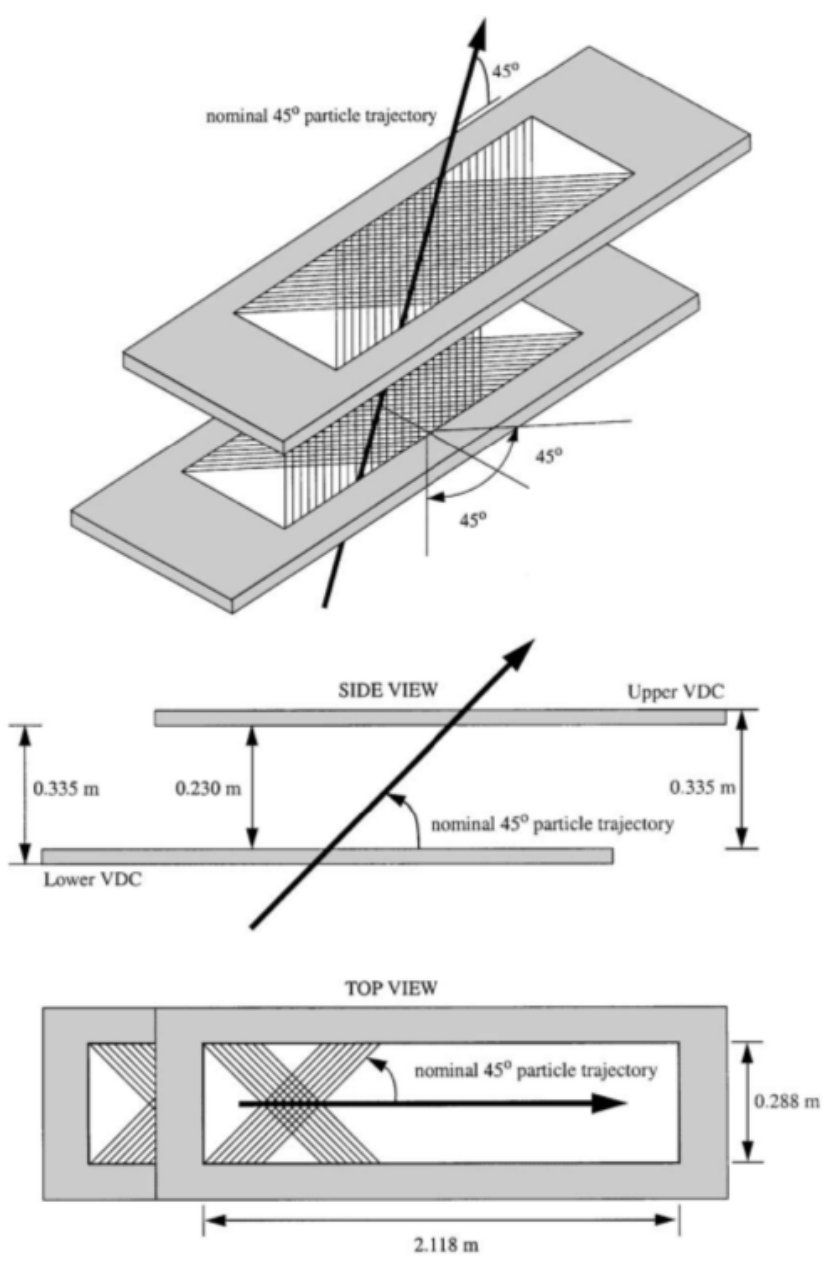

Figure 2.15: External VDC Diagram. Each of the two VDCs, called the upper VDC and lower VDC, contains two planes of 368 sense wires oriented at $45^{\circ}$ angles to each other. The interior of the VDCs is filled with a $62 \% / 32 \%$ argon/ethane gas mixture. A large electric field is created perpendicular to the wire planes which attracts electrons to the sense wires. Image from 15. 


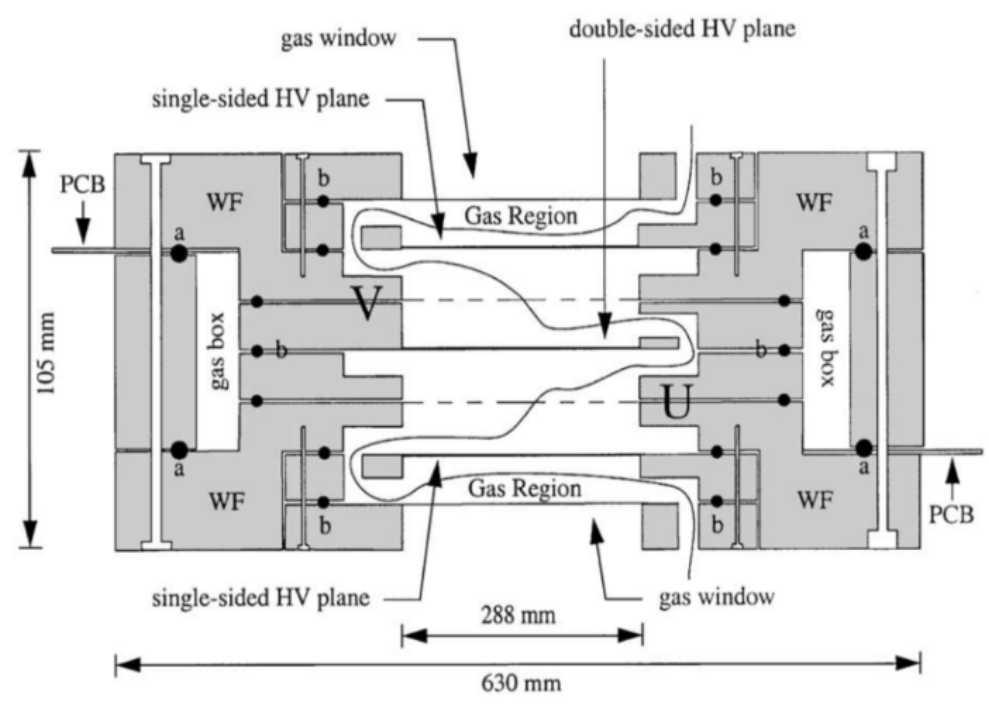

Figure 2.16: Internal VDC Side View Diagram. Two gold-plated mylar planes (three bold central lines) are situated above and below each sense wire plane (dashed lines). These mylar planes create a large a electric field perpendicular to the wire planes which attracts electrons to the sense wires. A $62 \% / 32 \%$ argon/ethane gas mixture is flowed through the VDCs from the gas boxes seen on either side of the figure. Image from [15].

When operating at a high enough voltage the sense wires are efficient to above $99 \%$ in the central region of the wire planes. Towards the edge of the wire planes the efficiency falls off. In general a charged particle will be detected by four to six wires. A wire is considered to be efficient if that wire fired at the same time as its two adjacent wires. Thus the efficiency of a wire is given by Equation 2.2, where $\kappa$ is the number of times a wire was considered to be efficient for an event and $\lambda$ is the number of times that the wire was inefficient for an event 15 . Figure 2.17 shows the wire efficiency spectrum for a good run on the left and a bad run on the right. The right spectrum indicates that the operating voltage may be too low or unsteady, and/or that some of the amplifier cards have become disconnected.

$$
\epsilon=\frac{\kappa}{\kappa+\lambda}
$$

Where and at what angle a charged particle passes through the VDCs determines 

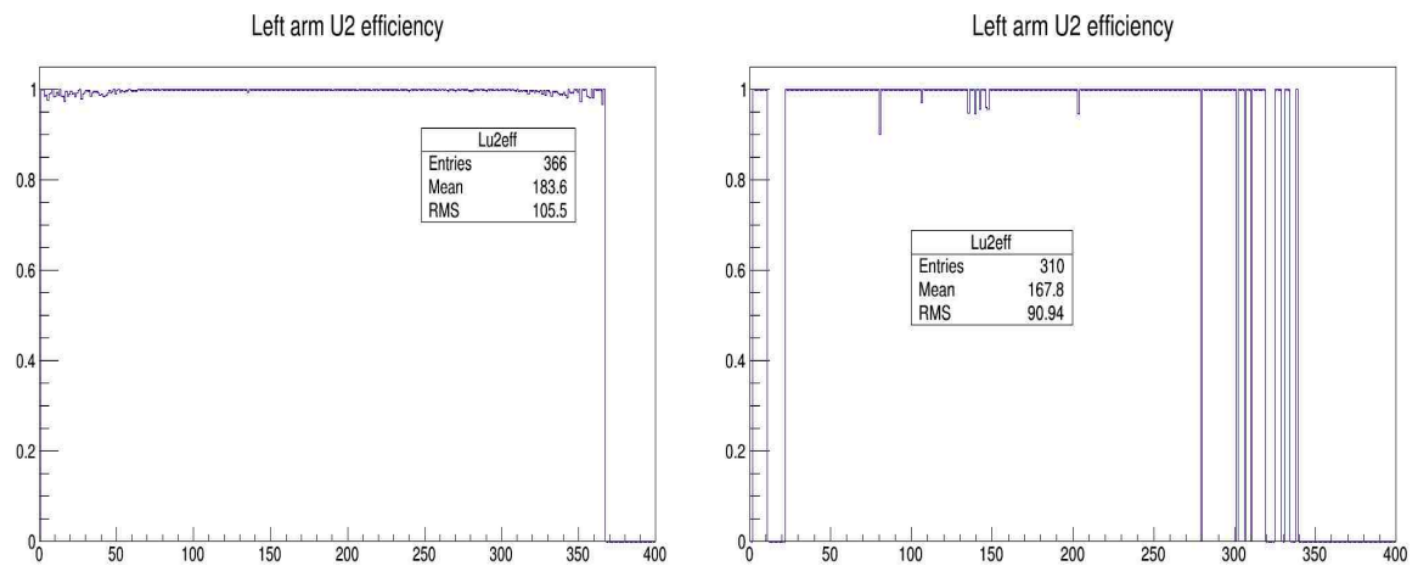

Figure 2.17: Example Wire Efficiency Spectra. The $X$-axis represents the wire number and the $Y$-axis represents the wire's efficiency. The left image indicates a good run, and the right image shows a bad run. In the right image the operating voltage may be too low or unsteady, and/or some of the amplifier cards have become disconnected.

the time it takes for the electrons produced by its passing to drift to the sense wires. Figure 2.18 shows a typical drift time spectrum for a single wire on the left. On the right it shows a single sense wire drift cell with the sense wire at the center of the circle. Timing is measured by a common stop TDC which triggers on the sense wire signals and is stopped by the main event trigger. Higher TDC channels indicate shorter drift times.

By examining each of the four trajectory regions A, B, C, and D on the right side of Figure 2.18 we can explain the structure of the drift time spectrum. Region A on the right side of the figure corresponds to TDC channels 1020-1080 on the left side of the figure. These drift times are created by electrons from particles with large trajectory angles that intersect less of the cell leading to a lower detection probability. Region B corresponds to channels 1080-1460. This region represents the bulk of the cell where the electric field lines are parallel giving the electrons a constant drift velocity and track density (i.e. the number of tracks that pass through a given region). Region C corresponds to channels 1460-1540. Here the field lines become quasiradial as we approach the sense wire. Electron drift velocity is roughly constant here, but the 
track density increases. Finally region D corresponds to channels 1540-1620. Now the trajectory is close to the sense wires so the electrons reach a maximal track density and a dramatic increase in drift velocity occurs because the electric field lines converge to the wire $[15$.
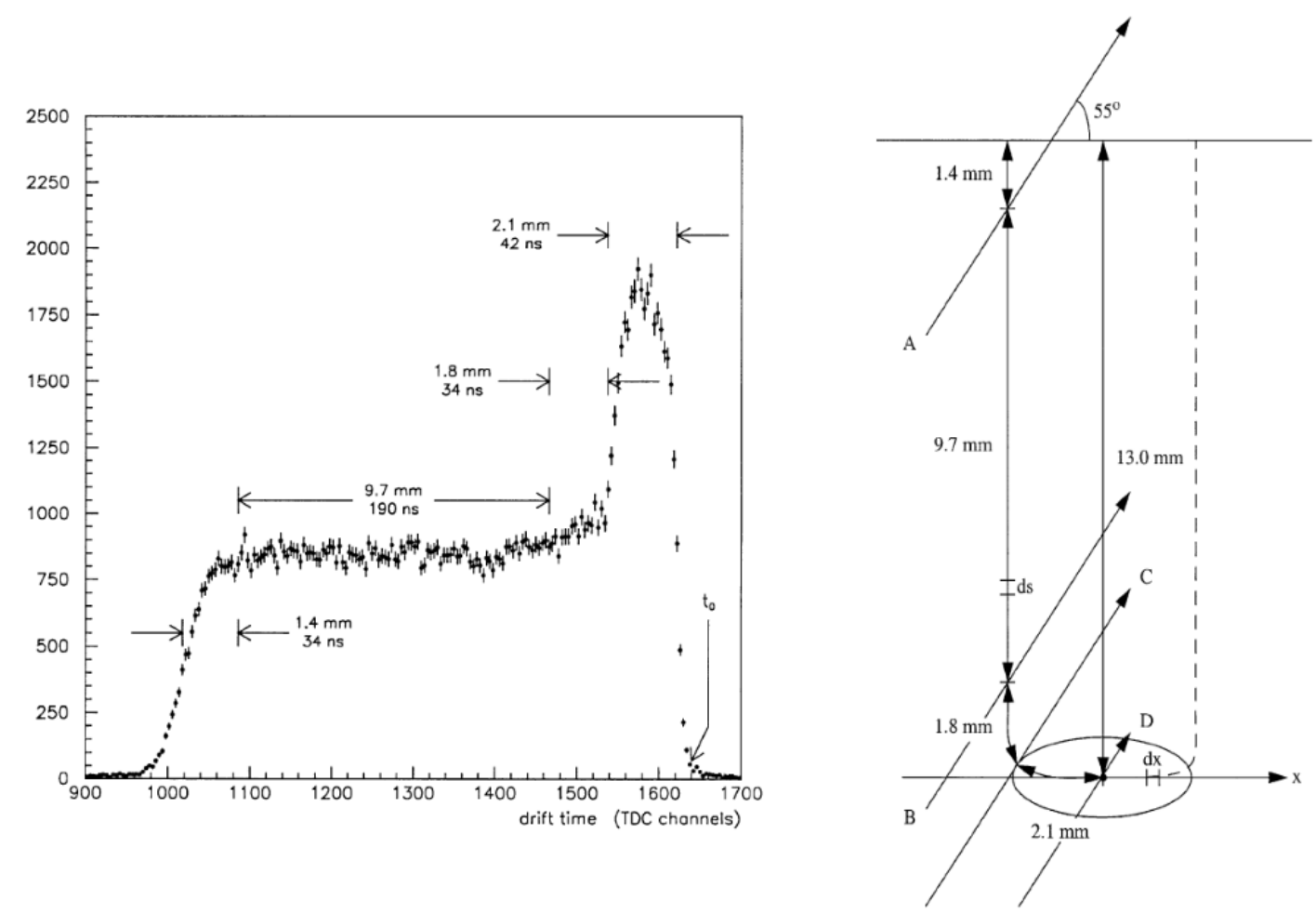

Figure 2.18: Wire Timing Spectrum. The left plot represents a single wire's timing spectrum. The $X$-axis is in units of TDC channel. The right image shows a single wire VDC drift cell and several trajectories a charged particle may take when passing through the cell. Image from 15.

The electron's drift time can be used to calculate the distance the particle was from the sense wire to begin pinning down its spatial location. Before this is done all of the drift spectra must be calibrated to one another. This is done by defining a reference time, $t_{0}$, which is defined as the channel where the maximal slope is obtained in the short drift time region around channel 1600. The drift distance can then be calculated by integrating over the number of events, $d N$, per time bin, $d t$, and scaled by a calibration constant, $k$, determined by the size of the drift cell as in Equation 2.3 
from $[15$.

$$
x\left(t^{\prime}\right)=\frac{1}{k} \int_{t_{0}}^{t^{\prime}} \frac{d N}{d t} d t
$$

The ultimate job of the VDCs is to reconstruct the charged particles' trajectories to provide $\theta, \phi$, and $\mathbf{k}^{\prime}$. A typical particle trajectory passes through all four wire planes in the two VDCs as shown in the left side of Figure 2.19. Because there are two wire planes per VDC the two locations where the particle was determined to cross each of the VDC's wire planes can be used to create a trajectory as seen in the right side of Figure 2.19. In this case the trajectory can be described by four variables where one has defined a $U-V$ plane starting from $(0,0)$ in the right side of the Figure [15].
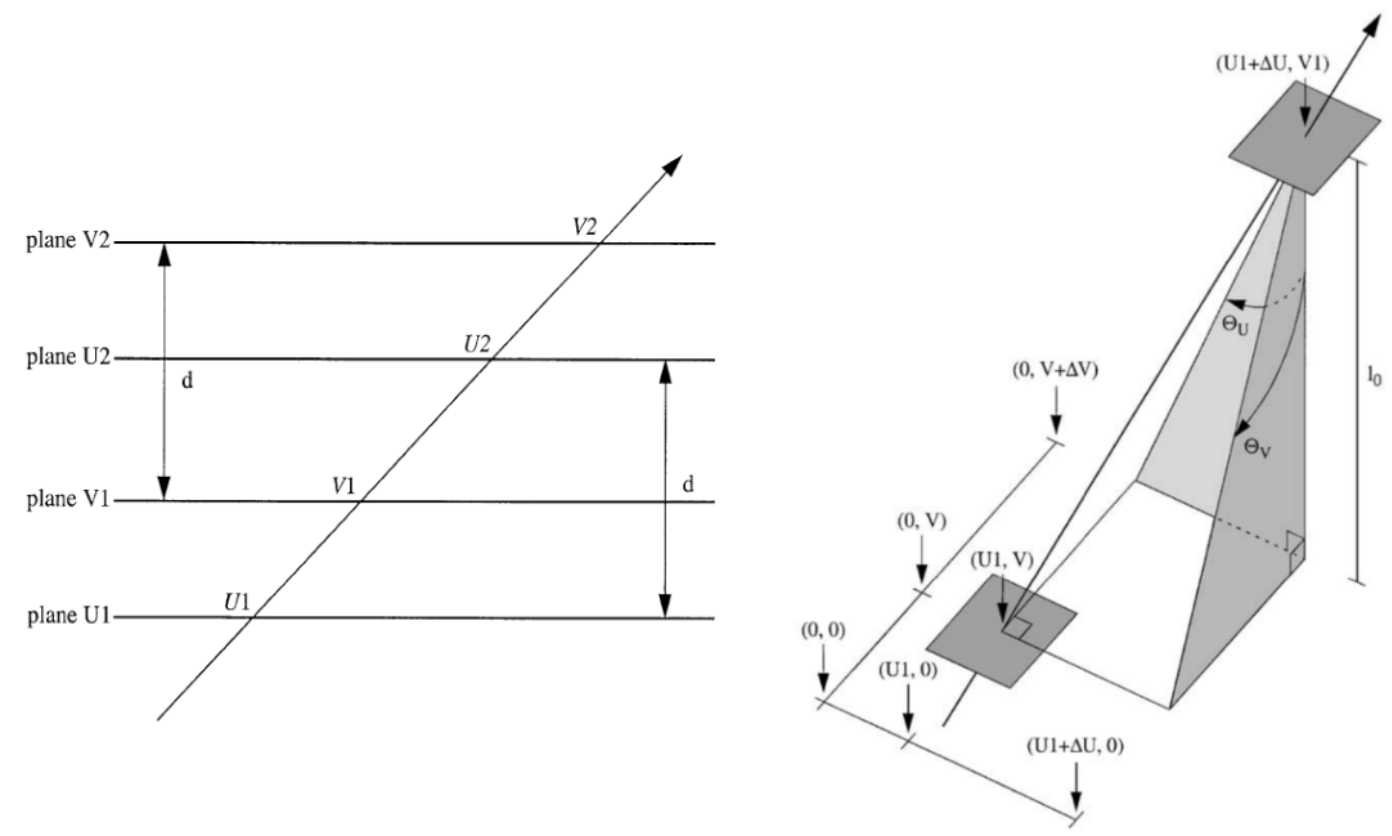

Figure 2.19: Trajectory Reconstruction. The left plot shows a charged particle passing through both VDCs, and the right plot shows the coordinates needed to reconstruct that particle's trajectory. Image from 15$]$.

The first two variables are the two $U-V$ coordinates of the crossing point of the particle through the first VDC wire plane called $(U, V)$. The second two variables are the two angles, $\theta_{U}$ and $\theta_{V}$, which are the angles in the $U$ and $V$ directions respectively 
between the two wire plane crossing points of the first VDC. Equations 2.4, 2.5, and 2.6 give these four variables with $\Delta U$ and $\Delta V$ being the differences between the crossing point locations in the $U$ and $V$ directions respectively. $d$ is the distance between the $U_{1}$ wire plane of the bottom VDC and the $U_{2}$ wire plane of the top VDC, and $l_{0}$ is the distance between the two wire planes in a single VDC[15] .

$$
\begin{gathered}
U=U_{1} \\
V=V_{1}-\Delta V=V_{1}-l_{0} \tan \left(\theta_{V}\right) \\
\tan \left(\theta_{U(V)}\right)=\frac{U_{2}-U_{1}}{d}
\end{gathered}
$$

\subsubsection{Scintillator Counters}

After the VDCs are two planes of scintillator paddles located two meters apart. Figure 2.20 shows a schematic of a single scintillator paddle. The first plane, $\mathrm{S}_{1}$, consists of six paddles of plastic scintillators which provide overlapping coverage. The second plane, $\mathrm{S}_{2 m}$, consists of 16 plastic scintillator paddles smaller than those in $\mathrm{S}_{1}$ [7. When a charged particle strikes one of these paddles it produces photons which travel along the length of the paddle to either end where the photons encounter a photomultiplier tube (PMT).

When the photons enter the PMT they create electrons via the photoelectric effect, and an electric field accelerates these electrons down the PMT creating a larger cascade of electrons as they pass through. This produces an analog electric signal. The PMT then passes this signal on to the data acquisition system. These scintillators are very efficient and have timing resolutions of around $0.30 \mathrm{~ns}[6][7]$. Due to these excellent timing responses most of the primary triggers in Hall $\mathrm{A}$ use a coincidence of $\mathrm{S}_{1}$ and $\mathrm{S}_{2 m}$ for the main production triggers of experiments. The triggers used in E08-014 are discussed in 2.9. 


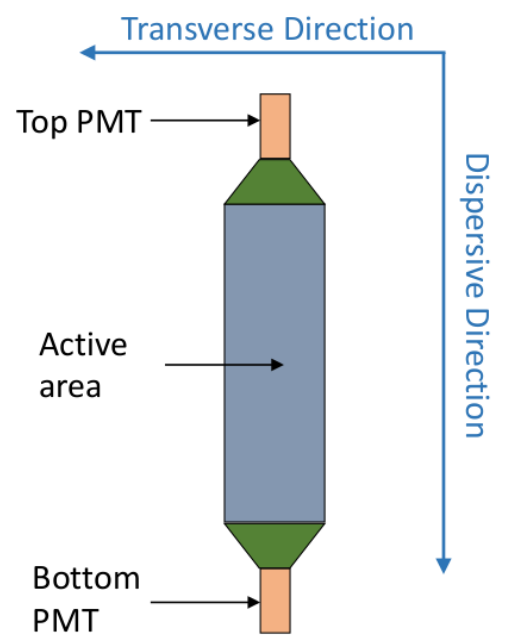

Figure 2.20: Single Plastic Scintillator Paddle. The $\mathrm{S}_{0}$ and $\mathrm{S}_{2 m}$ scintillator plane paddles are composed of an active plastic scintillator region for particle detection. The light released from the passage of particles then travels the length of the paddle to PMTs on either side. Image from [10].

\subsubsection{Gas Cherenkov}

Located in the detector stack between the $\mathrm{S}_{1}$ and $\mathrm{S}_{2 m}$ scintillator planes is a gas Cherenkov (GC) detector. The GC operates by detecting Cherenkov radiation created by particles passing through a gas at velocities greater than the speed of light in that gas medium. When light passes through a transparent medium its velocity is reduced by the medium's index of refraction, $n$, as seen in Equation 2.7. Because the speed of light is reduced, particles can travel faster than the speed of light in that medium. When a particle exceeds the speed of light in a medium (i.e. its velocity threshold), $v_{t h}$, it creates an electromagnetic shock wave in the same manner as a jet plane creating a sonic boom. The shock wave formed has the conical shape seen in Figure 2.21 where the angle $\theta$ is given in Equation 2.8 with $\beta=\frac{v}{c}$, where $v$ is the velocity and $c$ is the speed of light [16.

$$
v_{t h}=\frac{c}{n}
$$




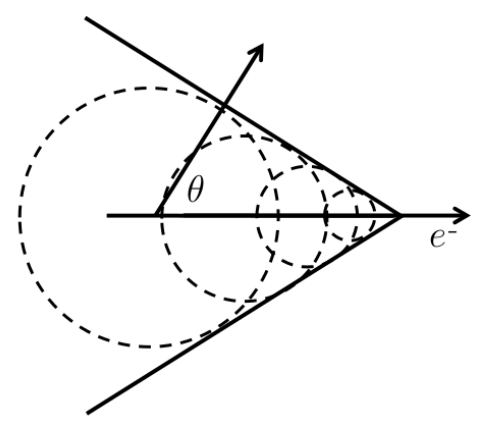

Figure 2.21: EM Shock Wave. The EM shock wave is produced by a particle exceeding the speed of light in the medium it is passing through. This shock wave then produces Cherenkov radiation. Image from [17].

$$
\cos (\theta)=\frac{1}{\beta n}
$$

GC detectors are especially useful for particle discrimination, in particular, for distinguishing between electron and pion events. GCs are able to discriminate between particles of different mass because of the velocity threshold required to create Cherenkov radiation shown in Equation 2.9. The Hall A GC is generally filled with $\mathrm{CO}_{2}$ which has an index of refraction of $n=1.00041$. Therefore, the momentum threshold for an electron is $p_{t h}=0.0178 \mathrm{GeV}$ and for a pion it is $p_{t h}=4.87 \mathrm{GeV}$. The HRSs accept momenta up to about $4.3 \mathrm{GeV}$. Thus electrons generally create a signal passing through the GC while the pions generally do not. It is still possible for pions to interact with the gas or the detector structures and create low energy $\delta$-electrons that can be seen by the GC, but this is a low probability process. When in normal operation the GC can generally detect electrons with an efficiency of over $99 \%$ ].

$$
p_{t h}=\frac{m c}{\sqrt{n^{2}-1}}
$$

The Hall A GCs each contain ten spherical mirrors which focus the Cherenkov light onto ten PMTs as seen in Figure 2.22. The LHRS GC has a detector path length of $80 \mathrm{~cm}$ and produces an average of 7 photoelectrons per event, and the RHRS GC has a detector path length of $130 \mathrm{~cm}$ and yields on average 12 photoelectrons per event. 
[6] [18]. Each PMT signal passes through a 10X amplifier before being split. One of these two signals is sent to an ADC and put in the data stream for offline analysis. The other signal is again split and one of these two new signals is converted to digital and sent to a TDC. The remaining PMT signal is summed with the PMT signals of the nine other PMTs. This summed signal is converted to a digital signal and then is used as part of the efficiency triggers. The combined GC signal was also added to the main production trigger during experiment E08-014 to prevent pions from firing the main trigger [7].

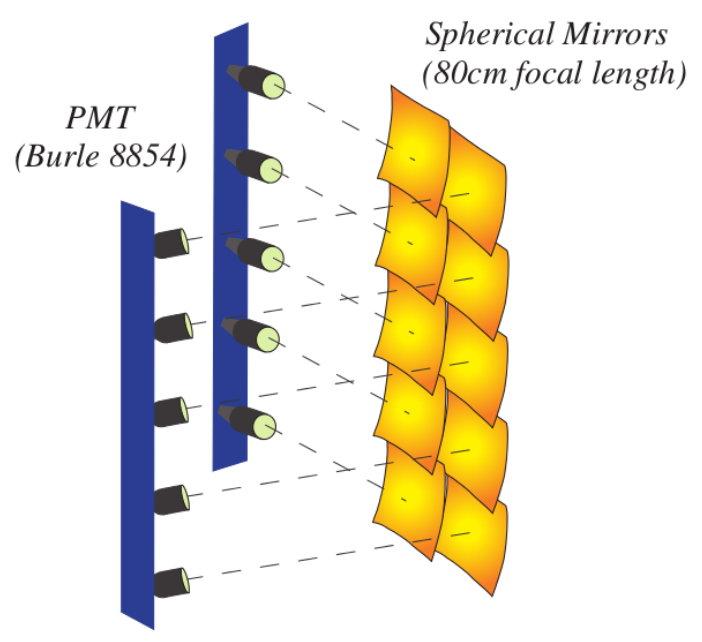

Figure 2.22: Hall A GC Interior. Ten spherical mirrors inside the gas Cherenkov reflect the Cherenkov light onto the ten PMTs. Image from [7].

The ten photomultiplier tubes in each GC must be calibrated such that they place the same charge detected in the same ADC channel. The charge detected is then proportional to the number of incident photons. This means each of the PMTs have the same response to a photon and thus can be compared to one another. This procedure has two parts, a hardware calibration and a software calibration. First the hardware is calibrated by gain matching the PMTs. This is done by first locating the single photoelectron (SPE) peak of each of the PMTs in the ADC data. Then by increasing or decreasing the voltage of the PMTs the SPE peak can be shifted up or down in ADC channels respectively. A target ADC channel is then chosen and the voltages 
of the individual PMTs are then adjusted until all of the SPE peaks are located in the same ADC channel. After the PMTs are gain matched they will each still have slightly different responses than the other tubes. The final software calibration is done by selecting the same target ADC channel for the SPE peaks. Each PMT's ADC spectrum is then given an constant offset to place its SPE peak in the desired channel. For the specific procedure and results from experiment E08-014's GC calibration see Section 4.2 .1 of $[7]$.

\subsubsection{Electromagnetic Calorimeters}

In the detector stack of each HRS behind the GC and the $\mathrm{S}_{2 m}$ scintillator plane sit a series of lead-glass blocks with PMTs attached making up an electromagnetic calorimeter. The calorimeters in the LHRS and RHRS have their lead-glass blocks arranged differently as shown in Figure 2.23. The LHRS blocks are set in two columns of 17 blocks each with the first layer called pion-rejector 1 (PRL1) and the second layer called pion-rejector 2 (PRL2). The RHRS blocks are arranged in two layers. The first layer is referred to as the 'preshower' and is made up of two columns of 24 PMTs each. The second layer, called the 'shower', is comprised of five columns of 16 blocks each [6] [7].

The calorimeters provide a second level of particle identification for the experiments. When charged particles pass through the lead-glass blocks they are slowed down by their interactions with the nuclei of the particles in those blocks (lead-glass is chosen for its heavy nuclei). The energy lost by this deceleration from nuclei is emitted as photons via Bremsstrahlung radiation. These released photons continue on and produce electron-positron pairs through pair production which again create more Bremsstrahlung radiation. This alternating process creates a 'shower' of photons, electrons, and positrons in the calorimeter. The PMTs then detect the Cherenkov light from these electrons and positrons in the lead-glass.

Heavier particles compared to electrons, like pions, require a much greater path length in the lead-glass blocks to release a shower of particles because pions mostly 
HRS-L
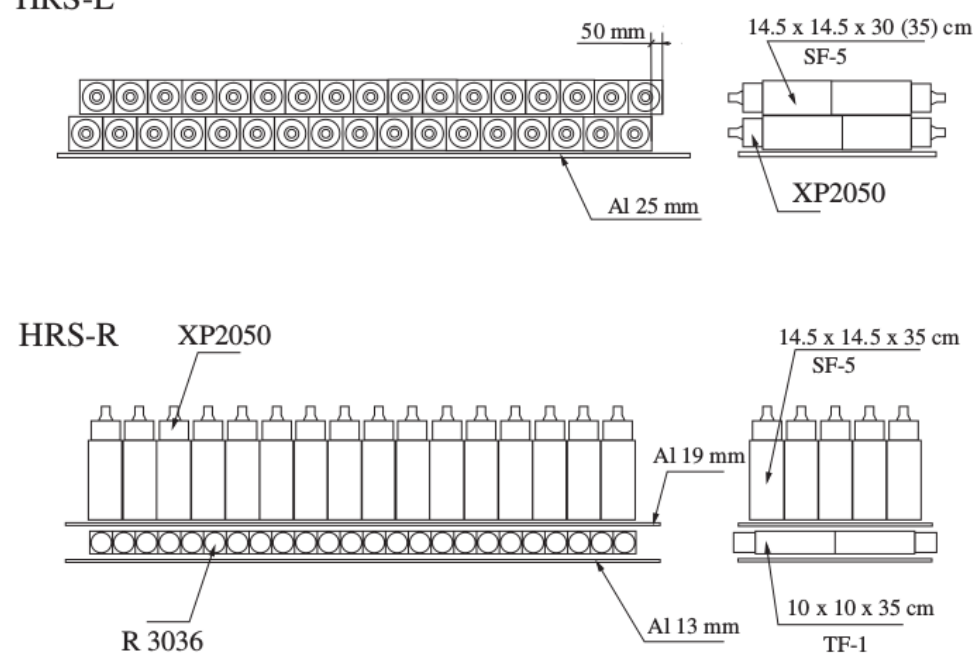

Figure 2.23: Hall A Electromagnetic Calorimeters. The LHRS blocks are set in two columns of 17 blocks each with the first layer called pion-rejector 1 (PRL1) and the second layer called pion-rejector 2 (PRL2). The RHRS blocks are arranged in two layers. The first layer is referred to as the 'preshower' and is made up of two columns of 24 PMTs each. The second layer, called the 'shower', is comprised of five columns of 16 blocks each [6] [7. Image from [6].

interact through ionization not Bremsstrahlung. This means that only electrons should create significant showers in the calorimeters. Although they still provide accurate particle identification the PRL1 and PRL2 in the LHRS do not totally absorb all of the electrons' energy, whereas the preshower and shower in the RHRS are total absorbers [7]. The PMTs attached to the lead-glass blocks must also be calibrated in the same manner as the GC discussed in 2.7.3. For a full accounting of the calorimeter calibration procedure for experiment E08-014 see section 4.2.2 of [7].

\subsection{Data Acquisition System}

A schematic of the data acquisition system (DAQ) is shown in Figure 2.24. To understand the Hall A DAQ let us follow the data signal chronologically through the various components. This journey begins with the analog signals produced by each of the detector components in the detector stack described in section 2.7. Some signals are digitized as they pass through the system. Detector signals enter the system 
through one of two ways. The first is by being sent into a majority logic unit (MLU) which then uses various combinations of signals to create triggers and coincidences between the detectors. For example, it may create a new trigger output that is a coincidence of the $\mathrm{S}_{1}, \mathrm{~S}_{2 m}$, and GC signals which was used as the main production trigger in experiment E08-014 [19].

\section{Schematic for Trigger System}

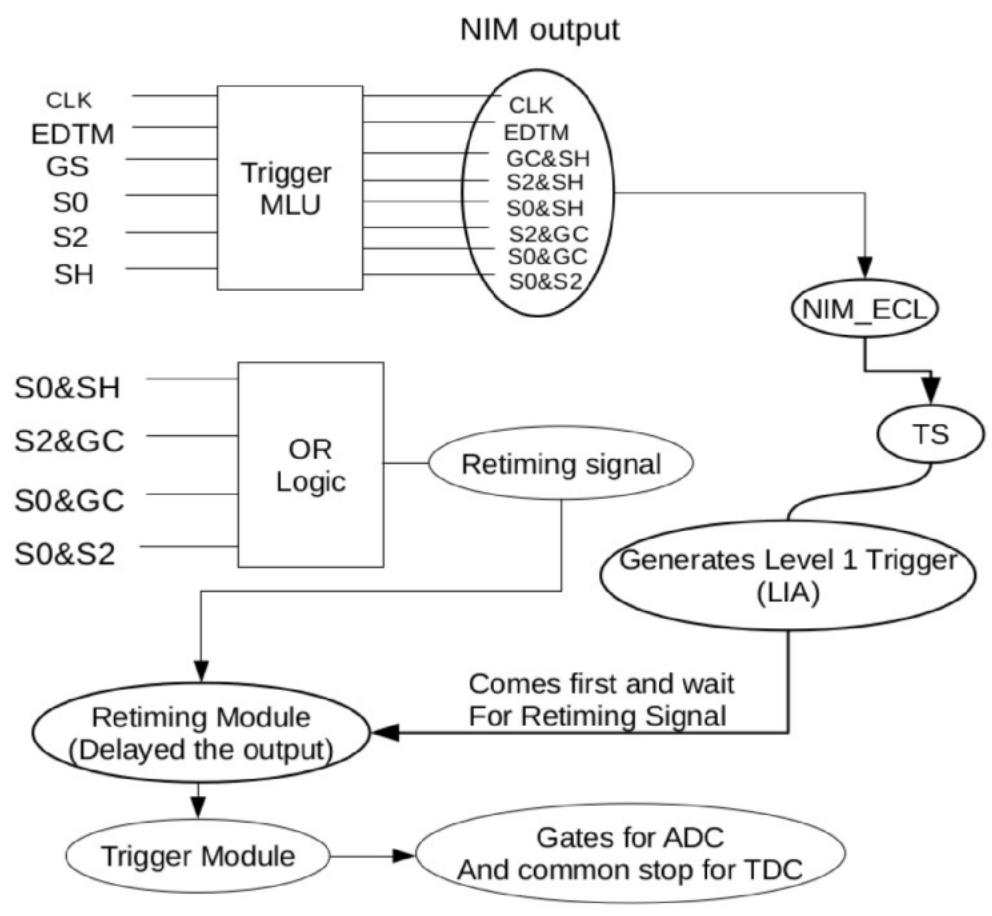

Figure 2.24: Schematic of Hall A DAQ and Trigger System. Image from 19.

These MLU output signals are then transported via NIM-ECL translator to a trigger supervisor (TS) module. The TS module is the central control point for the DAQ. This module decides whether or not to accept a trigger from the detectors. If a trigger is accepted the TS then creates a level one accept (L1A) signal. These L1A signals provide timing and gating information for the electronics such as ADCs and TDCs. The TS can also prescale the triggers if the rate is higher than desired. The L1A signals are then sent to the retiming module where they await the trigger signals from the detectors that enter the DAQ via the second method [19]. 
The second way into the DAQ is by taking the signals from the detectors directly into NIM cards. Here, using the NIM cards such as amplifiers and coincidence modules, the detector signals are combined to make triggers matching those produced by the MLU. These signals are called the retiming signals and are also sent to the retiming module where they meet up with the L1A signals. The L1A signals are then retimed to match the detector signals. Now the gates and stops determined by the detector signals are in coincidence with the L1A signal 19 .

This coincidence signal is sent to the transition module (TM) which acts as an interface between the TS and the electronics. The TM then copies the signals for the triggers, gates, and stops and sends them to the VME and FASTBUS crates containing the ADCs, TDCs, and scalers which are controlled by read out controllers (ROCs). These front end electronics then begin recording data based on the trigger information from the detectors and a unique event number for this trigger is created that contains all of the front end electronics information for that event [19].

The Hall A DAQ also contains two timing scalers (clocks), a fast (104 kHz) and a slow clock $(1024 \mathrm{~Hz})$, which are used to time the experimental run and are used to normalize experimental data. The timing from the clocks allows the missing data from computer dead-time to be understood. When high data rates are passing through the DAQ the computer processing time of that data becomes a limiting factor. While one event is being processed the next event that arrives before the first event is processed is missed by the system and not recorded. This problem can be alleviated in several ways.

The first method is to 'prescale' the data meaning that the system doesn't try to read all of the data. For example if the system is set to a prescale factor of five the system will only record one fifth of the data in the stream. This allows the electronics to keep up and the data can be normalized back by knowing the prescale factor. The proportion of time the DAQ was able to accept signals is known as the 'live time' given in Equation 2.10. Here $p s_{i}$ is the prescale value of trigger $i, T_{i}^{a c c}$ is the number of trigger $i$ accepted and recorded by the DAQ, and $T_{i}$ is the total number of trigger $i$ 
created by the detectors. The 'dead-time' is then equal to one minus the live time. [19].

$$
L T=\frac{p s_{i} \times T_{i}^{a c c}}{T_{i}}
$$

Another method of preventing data from being lost to electronic dead-time is by placing the data in a buffer before analyzing it. The buffer is essentially an electronic storage bin where the raw data is kept in computer memory until the system has processed all the events preceding the buffered event. As long as the buffer is large enough all the events can be processed eventually without loss. This allows the experiment to run as fast as the front end electronics are capable of running [19].

The dead-time can be monitored by several methods. One is by the electronic dead-time monitor (EDTM) signal in the DAQ. The EDTM sends constant pulser signals into the $\mathrm{S}_{1}, \mathrm{~S}_{2 m}$, and GC data streams. If the DAQ is free these signals will be accepted, but if the DAQ is busy they will be rejected. The number of accepted signals in the final data can be compared to the known EDTM pulser rate to calculate the dead-time. The TS module also sends out an electronic busy signal when it is processing data and unable to accept new triggers. This busy signal is essentially an internal constant pulser signal that is gated by the TS entering the busy state, and provides a second method of calculating the dead-time [19].

Now we have seen how the data is gathered from the detectors, but how is this system controlled and how does the data come to be in a datafile useful for analysis? The CEBAF Online Data Acquisition (CODA) program, seen schematically in Figure 2.25, allows the DAQ to be controlled remotely and builds the experiment's run datafiles. The main CODA GUI is used to begin and end each experimental run. The GUI communicates these commands to a readout controller (RC) server that ties all of CODA's components together. Once the RC server gets the command to begin a run it informs the electronics described above to begin the process of taking data 19 .

Once a trigger is accepted by the DAQ the crates containing ADCs and TDCs are 


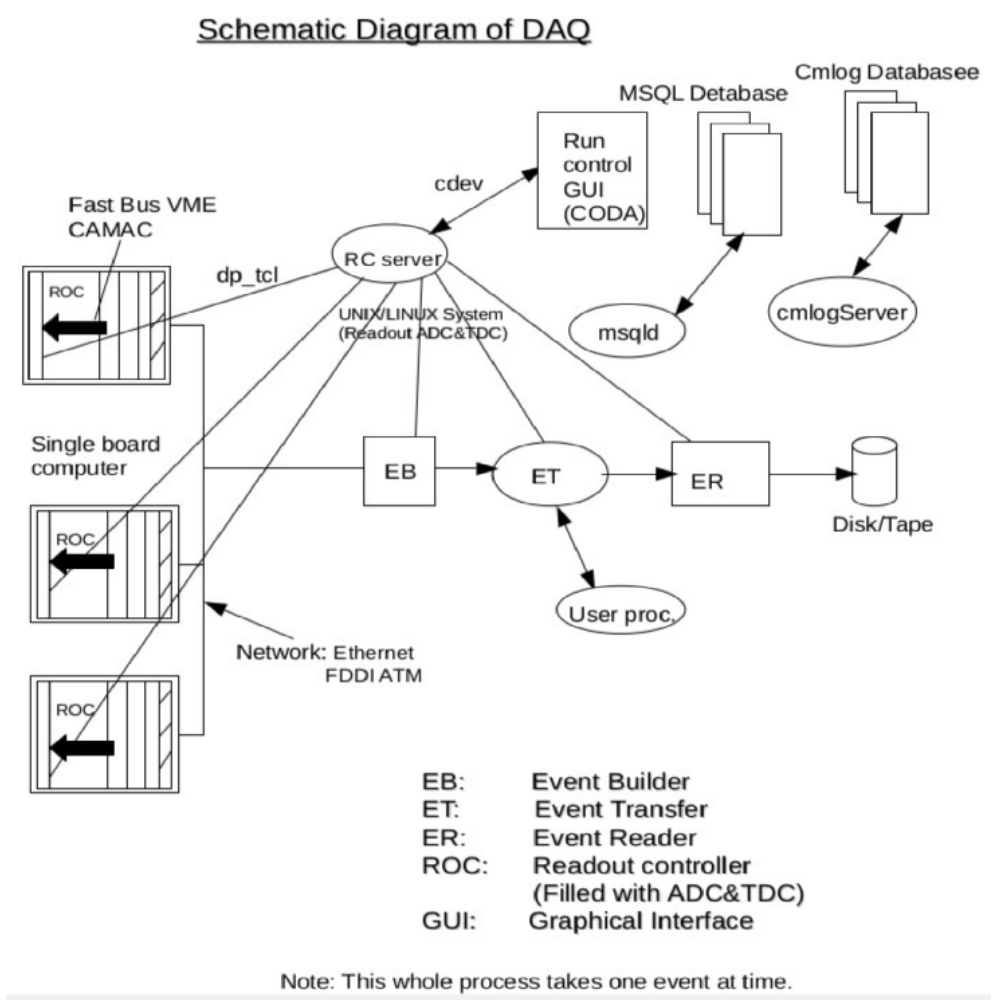

Figure 2.25: Schematic of CODA. Image from 19 .

read out by the ROCs. The ROCs pass this information on to CODA's event builder (EB). The EB then takes these disparate pieces of data from the various components and organizes them all into one file using the CODA formatting structure. The EB then sends this file in the form of a single event to the event transfer (ET) system. The ET gathers these events and then sends them on to the event recorder (ER) where they are finally written to permanent storage such as tape file. The resulting CODA files can then be decoded to create ROOT files which are then used in the offline analysis of the experimental runs. These data files contain scaler readouts every one to four seconds. They also log the Experimental Physics and Industrial Control System (EPICS) data, which contains information from the hall like target position, spectrometer angle, BCM readings, BPM readings, beam energy, and spectrometer magnet information, periodically every few seconds 7 [19]. 


\section{$2.9 \quad$ Triggers}

Experiment E08-014 used seven different triggers. Each of these triggers is made up of a combination of the signals from the $\mathrm{S}_{1}$ and/or $\mathrm{S}_{2 m}$ scintillator planes and the sum of the ten GC signals. The main production trigger is a coincidence of $\mathrm{S}_{1}, \mathrm{~S}_{2 m}$, and the GC sum signals called T1 (T3) for the RHRS (LHRS), and is denoted by $\left(\mathrm{S}_{1}\right.$ $\& \mathrm{~S}_{2 m}$ \& GC). Two efficiency triggers designed to measure the efficiency of the main production triggers T1 (T3) are T2 (T4) for the RHRS (LHRS). These are made up of a coincidence of the GC signal and only one of either $\mathrm{S}_{1}$ or $\mathrm{S}_{2 m}$. The triggers $\mathrm{T} 6$ (T7) for the RHRS (LHRS) are the coincidences of the $\mathrm{S}_{1}$ and $\mathrm{S}_{2 m}$ scintillator planes. Since these triggers do not involve the GC they recorded pion events as well and were thus useful for particle identification purposes. Finally trigger T5, the coincidence of T1 and T3, was disabled for this experiment 7 .

\subsection{High Resolution Spectrometer Optics}

Once the charged particles are bent through a spectrometer and into the detector stack they first pass through the two VDCs set in the HRS's focal plane as described in 2.7.1. The VDCs give the particle's location in the focal plane which can then be used to reconstruct where the electron interacted with the target, the 'reaction vertex', as well as its trajectory at the target. This reconstruction is done by applying an optics matrix determined by the characteristics of the HRS. This matrix needs to be calibrated for each experiment as there are always slight changes in the relative positions of the target, spectrometer, and detectors as well as changes in spectrometer magnet behavior. This section describes the coordinate systems used in Hall A and the standard procedure for optimizing the optics matrix.

\subsubsection{Hall A Coordinate Systems}

Hall A has five different coordinate systems which can all be related to one another.

- Hall Coordinate System (HCS): 
The HCS can be seen in Figure 2.26. The origin of the HCS begins at the center of Hall A which is located at the intersection of the electron beam and the target's vertical axis of symmetry, $\hat{x}$. The $\hat{z}$ direction is defined in the direction of the electron beam's travel. The $\hat{y}$ direction is defined to be vertically up [20].

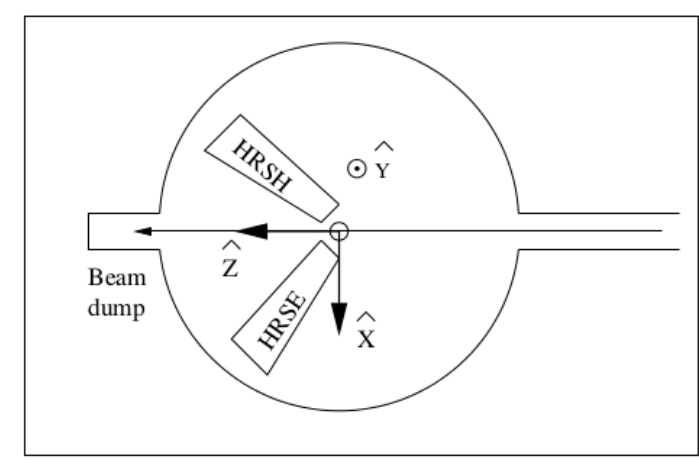

Hall Coordinate System (top view)

Figure 2.26: Hall Coordinate System. Image from 20.

\section{- Target Coordinate System (TCS):}

Each spectrometer has their own target coordinate system as seen in Figure 2.27. The $z$ axis is defined by drawing a line perpendicular from the sieve slit surface of the spectrometer and the midpoint of the central sieve slit hole. The $\hat{z}_{t g}$ direction is defined to be pointing away from the target. When optimally aligned the spectrometer is pointing at the hall center with the sieve slit being perfectly centered causing the $\hat{z}_{t g}$ vector to pass through the hall center 20].

With this optimal alignment the distance separating the hall center and the central midpoint of the sieve slit hole is defined as the spectrometer constant, $Z_{0}$. By definition the TCS origin is located a distance $Z_{0}$ from the sieve surface on the $\hat{z}_{t g}$ axis, and in the optimal case is the same as the hall center. The $\hat{x}_{t g}$ direction is defined to be parallel to the sieve plate surface pointing downwards. Finally the in-plane and out-of-plane angles, $\phi_{t g}$ and $\theta_{t g}$, are defined as $\frac{d y_{t g}}{Z_{0}}$ and $\frac{d x_{t g}}{Z_{0}}$ respectively 20 .

- Detector Coordinate System (DCS):

The origin of the detector coordinate system is located inside of the first VDC 


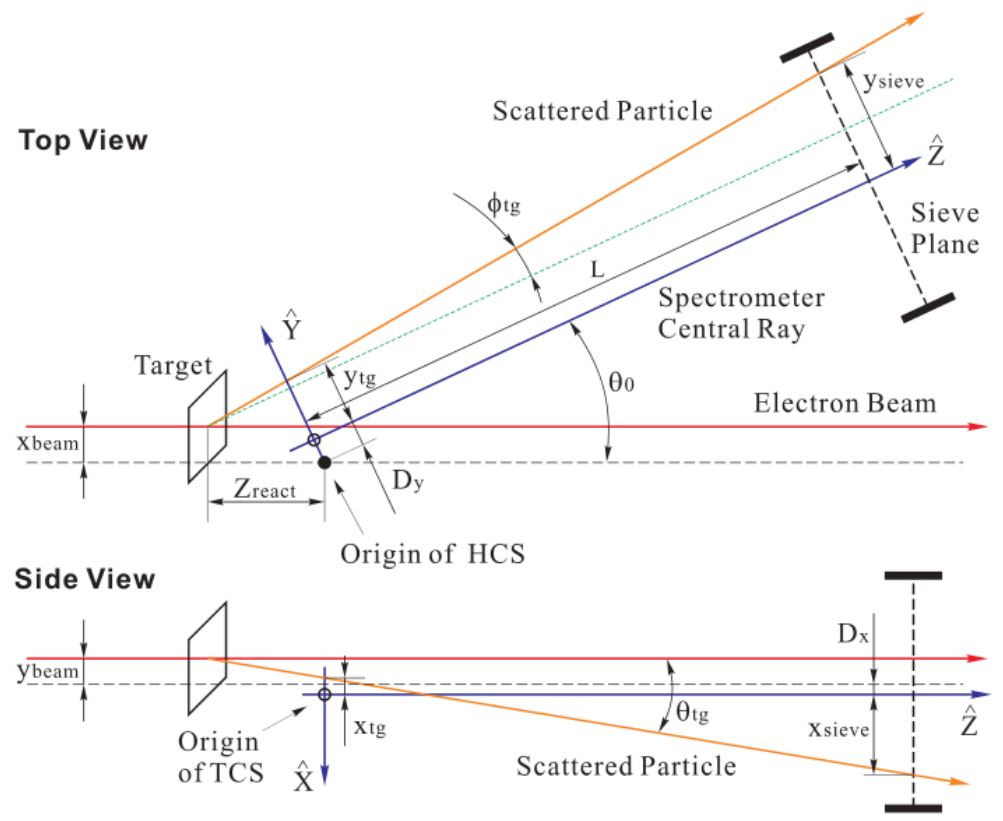

Target coordinate system (TCS). $\hat{z}$ goes from the target system perpendicularly to the center hole of the sieve slit plane attached to the Q1 entrance of each HRS. The intersection of $\hat{z}$ and the vertical axis of the target system defines the origin, hence there is a potential offset between the hall center and the origin of TCS. $\hat{x}$ is normal to $\hat{z}$ and points down, and $\hat{y}$ is to the left of $\hat{z}$.

Figure 2.27: Target Coordinate System. Image from 7 .

on either spectrometer as shown in 2.28 . This origin is defined by the intersection of wire 184 at the center of the U1 wire plane of the bottom VDC with the projection of wire 184 at the center of the V1 wire plane of the bottom VDC. $\hat{y}$ is defined in the direction parallel to the short axis of the VDC pointing to the left of the direction of the particles entering the VDC. $\hat{x}$ is defined along the longer VDC axis pointing away from the hall center. The $\hat{z}$ direction is defined as vertically up. For a more detailed description of the coordinates and how to calculate the detector vertex see [20].

\section{- Transport Coordinate System (TRCS):}

The transport coordinate system is defined by rotating the DCS $45^{\circ}$ about its $\hat{y}_{\text {det }}$ axis as shown in Figure 2.29 20].

\section{- Focal Plane Coordinate System (FCS):}

The FCS is another rotated coordinate system as shown in Figure 2.30. It is created by rotating the DCS about its $\hat{y}_{\text {det }}$ axis by and angle $\rho . \rho$ is defined as the 


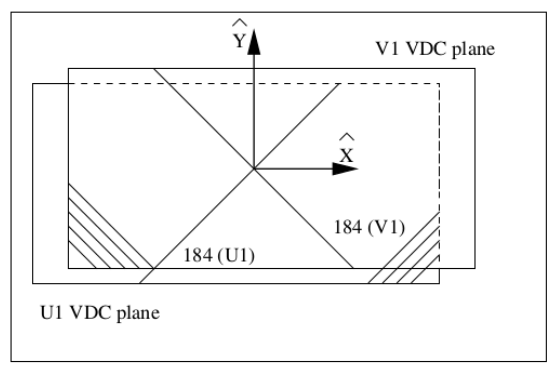

Detector Coordinate System (top view)

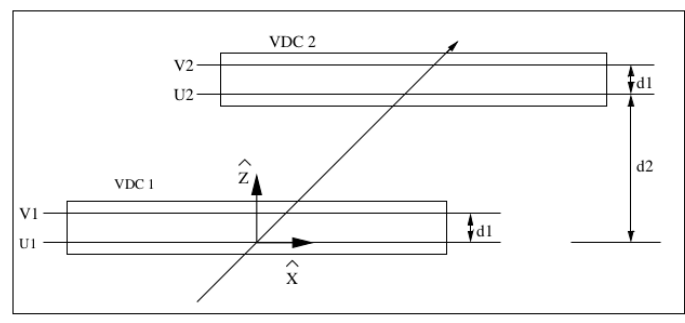

Detector Coordinate System (side view)

Figure 2.28: Detector Coordinate System. Image from [20].

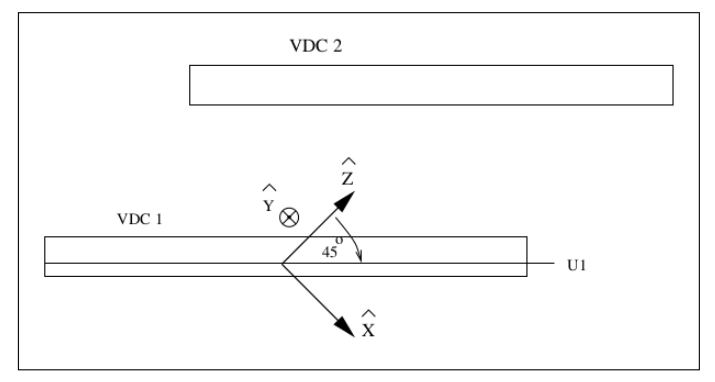

Transport Coordinate System (side view).

Figure 2.29: Transport Coordinate System. Image from 20].

angle between the $\hat{z}_{\text {det }}$ axis and the central ray passing through the target, i.e. $\phi_{t g}=$ $\theta_{t g}=0$, for the corresponding relative momentum given in Equation 2.11 [7] 20].

$$
\delta p=\frac{p-p_{0}}{p_{0}}
$$

\subsubsection{Spectrometer Optics Optimization Procedure}

Now that we understand the coordinate systems in Hall A we must calibrate the optics of the HRS so that we can reconstruct what happens at the target when the 


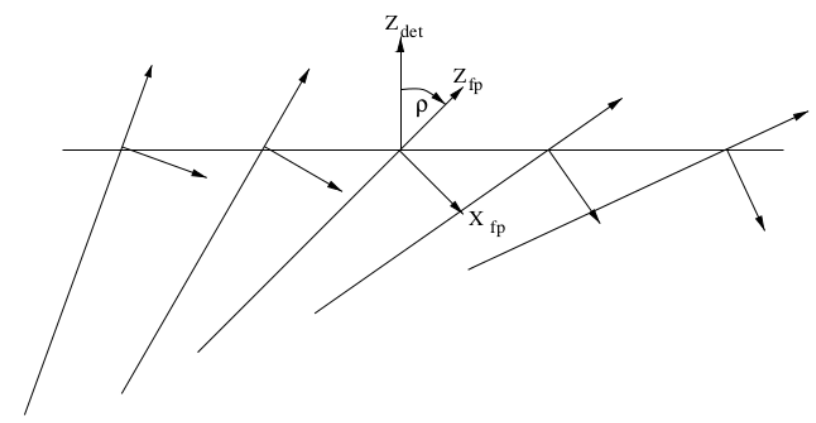

The focal plane (rotated) coordinate system as a function of the focal

Figure 2.30: Focal Plane Coordinate System. Image from 20.

electrons scatter. To do this we create an optics matrix that links the focal plane coordinates to the target coordinates. To first order this matrix can be written as Equation 2.12, A set of polynomial tensors in $x_{f p}$ can then describe the target variables in terms of the focal plane variables as shown in Equations 2.13, 2.14, 2.15, and 2.16, These tensors can all be written similar to the one written in Equation 2.17 [20].

$$
\begin{gathered}
{\left[\begin{array}{l}
\delta \\
\theta \\
y \\
\phi
\end{array}\right]_{t g}=\left[\begin{array}{cccc}
\langle\delta \mid x\rangle & \langle\delta \mid \theta\rangle & 0 & 0 \\
\langle\theta \mid x\rangle & \langle\theta \mid \theta\rangle & 0 & 0 \\
0 & 0 & \langle y \mid y\rangle & \langle y \mid \phi\rangle \\
0 & 0 & \langle\phi \mid y\rangle & \langle\phi \mid \phi\rangle
\end{array}\right]\left[\begin{array}{l}
x \\
\theta \\
y \\
\phi
\end{array}\right]_{f p}} \\
\delta=\sum_{j, k, l} D_{j, k, l} \theta_{f p}^{j} y_{f p}^{k} \phi_{f p}^{l} \\
\theta_{t g}=\sum_{j, k, l} T_{j, k, l} \theta_{f p}^{j} y_{f p}^{k} \phi_{f p}^{l} \\
y_{t g}=\sum_{j, k, l} Y_{j, k, l} \theta_{f p}^{j} y_{f p}^{k} \phi_{f p}^{l} \\
\phi_{t g}=\sum_{j, k, l} P_{j, k, l} \theta_{f p}^{j} y_{f p}^{k} \phi_{f p}^{l}
\end{gathered}
$$




$$
D_{j, k, l}=\sum_{i=1}^{m} C_{i}^{D_{j, k, l}} x_{f p}^{i}
$$

This matrix is calibrated by placing a sieve slit, shown in Figure 2.31, over the spectrometer entrance. The sieve has a series of holes with a well known pattern. This pattern then shows up in the focal plane data and by knowing the hole locations well the focal plane data can be correlated with the sieve holes. The variables in Equations 2.14, 2.15, and 2.16 described above turn out to be impractical to work with so an additional three variables are defined.

The first of these variables is $Z_{\text {react }}$ which describes the point of interaction between the beam and the target given in Equation 2.18. The second and third are $x_{\text {sieve }}$, Equation 2.19, and $y_{\text {sieve }}$, Equation 2.20, which describe the horizontal and vertical positions of the sieve plate respectively. In these equations $L$ and $D_{y}$ are defined as they were in the TCS above, $\theta_{0}$ is the spectrometer angle, and $x_{\text {beam }}$ is the horizontal beam position [20]. For the specific results of the optics calibration procedure for experiment E08-014 see section 4.3.2 of [7].

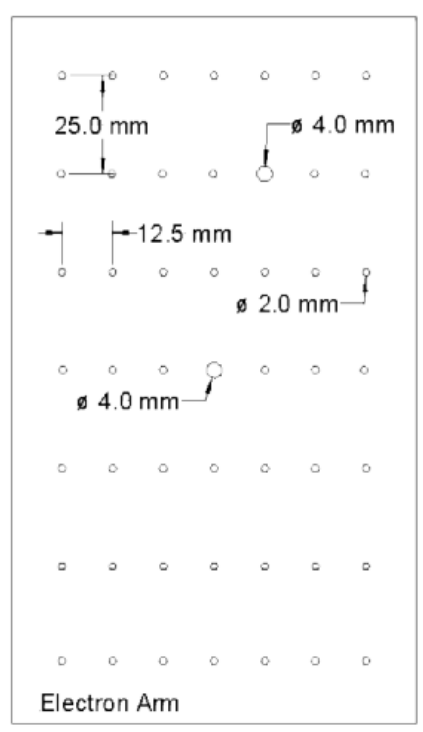

Figure 2.31: Optics Sieve Plate for E08-014. The two larger holes make it possible to determine the plate's orientation when performing the optics calibration. Image from 7 . 


$$
\begin{gathered}
Z_{\text {react }}=\frac{-\left(y_{t g}+D_{y}\right)+x_{\text {beam }}\left(\cos \left(\theta_{0}\right)-\phi_{t g} \sin \left(\theta_{0}\right)\right)}{\phi_{t g} \cos \left(\theta_{0}\right)+\sin \left(\theta_{0}\right)} \\
x_{\text {sieve }}=x_{t g}+L \theta_{t g} \\
y_{\text {sieve }}=y_{t g}+L \phi_{t g}
\end{gathered}
$$




\section{Chapter 3}

\section{Cross Section Extraction}

\section{$3.1 \quad$ Overview}

This chapter will explore the various analyses used to extract a ${ }^{3} \mathrm{He}$ elastic cross section from experiment E08-014's data. This discussion will include all measurements required to extract a differential cross section, various corrections for efficiency losses, the physics cuts applied to the data, and an estimate of the uncertainties. Also discussed will be the Monte Carlo software used to create an artificial elastic electron spectrum as well as the software used to calculate radiative corrections to this data.

\subsection{Experimental Cross Section}

The theoretical origins of a differential cross section were explored in Section 1.2 . However, this derivation is not particularly useful for extracting an actual experimental cross section. Extracting a cross section is essentially an exercise in electron counting. Let us now write the cross section as an experimentalist understands it as in Equation 3.1. Each of these variables will be examined in detail later in this chapter.

$$
\left(\frac{d \sigma}{d \Omega}\right)_{\text {exp }}=\frac{p s N_{e}}{N_{\text {in }} \rho L T \epsilon_{\text {det }}} \frac{1}{\Delta \Omega \Delta P \Delta Z}
$$

Here $p s$ represents the prescale value of the given trigger being examined. $N_{e}$ is 
the number of electrons detected by the experiment that survive physics cuts, like particle identification, and acceptance cuts. $N_{i n}$ is the number of electrons incident on the target, and can be calculated from the cumulative beam charge. $\rho$ is the target's density. $L T$ is the livetime correction which accounts for events missed due to electronic dead-time in the DAQ. $\epsilon_{\text {det }}$ represents the product of all of the detector efficiencies such as the GC, VDC single track, EM calorimeter, and trigger efficiencies. $\Delta \Omega$ represent the solid angle covered by the spectrometer after acceptance cuts. $\Delta P$ is the momentum acceptance seen by the spectrometer after acceptance cuts. Finally $\Delta Z$ represents the length of the target seen by the spectrometer.

\subsection{Beam Charge}

First we begin by finding the charge of the electron beam during any given experimental run. This process begins with the U and D BCMs measuring the beam current prior to the beam striking the target. These BCMs are calibrated according to Equation 3.2, and the constants can be found in Table 3.1. The BCM calibration for experiment E08-014 can be read about in more detail in [14 where these equations are found. The final constants in the table were updated after the analysis in [14], and were provided via private communication with Dien Nguyen.

$$
\left\langle I_{\text {beam }}\right\rangle=\frac{\frac{\text { scaler }}{\text { time }}-K_{\text {offset }}^{\prime}}{C_{V-\text { to-F }}}
$$

\begin{tabular}{|ccc|}
\hline BCM & $\boldsymbol{K}_{\text {off set }}^{\prime}$ & $\boldsymbol{C}_{\boldsymbol{V}-\text { to-F }}$ \\
\hline$U_{1}$ & 200 & 1035 \\
$D_{1}$ & 37 & 1263 \\
\hline
\end{tabular}

Table 3.1: BCM Calibration Constants for E08-014

The average current, $\left\langle I_{\text {beam }}\right\rangle$, is thus dependent upon the number of counts in the scaler measuring beam current in a certain period of time tracked by a clock scaler which is then modified by the two known calibration constants $K_{o f f s e t}^{\prime}$ and $C_{V-t o-F}$. Now that we have a current we can calculate the charge, $Q$, of the beam during a run 
using Equation 3.3. We can then divide the charge by the elementary charge of the electron, $e$, to find the total number of electrons incident on our target, $N_{i n}$, as in Equation 3.4

$$
\begin{gathered}
Q=\left\langle I_{\text {beam }}\right\rangle \times \text { time } \\
N_{\text {in }}=\frac{Q}{e}
\end{gathered}
$$

Figure 3.1 shows the current as measured by the $U_{1}$ and $D_{1}$ BCMs for experimental run 4074. During E08-014 the target beam current was $120 \mu \mathrm{A}$. When the beam 'trips' (abruptly turns off) and when the beam current is being brought up to the desired current it tends to be somewhat unstable. These events can be seen in the trailing edges and leading edges of the gaps in the BCM current measurement. These periods of instability are discarded by placing cuts on the current spectrum represented by the red lines in Figure 3.1. These lines are placed two scaler readouts, about 4 seconds per readout, before (after) the BCMs register $90 \%$ of the $120 \mu \mathrm{A}$ operating current for the falling (rising) edge. This analysis was performed for each run of Kin 3.2 and the

\begin{tabular}{|c|c|c|c|c|c|c|}
\hline Run & $\begin{array}{c}\text { Charge } \\
U_{1} \\
(\mu C)\end{array}$ & $\begin{array}{c}\text { Charge } \\
D_{1} \\
(\mu C)\end{array}$ & $\begin{array}{c}\text { Average } \\
\text { Charge } \\
(\mu C)\end{array}$ & $\begin{array}{c}\text { Electrons } \\
\quad U_{1}\end{array}$ & $\begin{array}{c}\text { Electrons } \\
D_{1}\end{array}$ & $\begin{array}{l}\text { Average } \\
\text { Electrons }\end{array}$ \\
\hline 3892 & 5568.68 & 6015.81 & 5792.25 & $3.47608 \times 10^{16}$ & $3.75519 \times 10^{16}$ & $3.61564 \times 10^{16}$ \\
\hline 3893 & 118261 & 118016 & 118138 & $7.38207 \times 10^{17}$ & $7.36678 \times 10^{17}$ & $7.37443 \times 10^{17}$ \\
\hline 3894 & 136502 & 138131 & 137316 & $8.5207 \times 10^{17}$ & $8.62243 \times 10^{17}$ & $8.57157 \times 10^{17}$ \\
\hline 4073 & 7656.35 & 7654.74 & 7655.54 & $4.77924 \times 10^{16}$ & $4.77824 \times 10^{16}$ & $4.77874 \times 10^{16}$ \\
\hline 4074 & 251551 & 251227 & 251389 & $1.57023 \times 10^{18}$ & $1.56821 \times 10^{18}$ & $1.56922 \times 10^{18}$ \\
\hline 4075 & 280417 & 280017 & 280217 & $1.75042 \times 10^{18}$ & $1.74792 \times 10^{18}$ & $1.74917 \times 10^{18}$ \\
\hline Totals & 799956 & 801062 & 800509 & $4.99348 \times 10^{18}$ & $5.00039 \times 10^{18}$ & $4.99693 \times 10^{18}$ \\
\hline
\end{tabular}
results are shown in Table 3.2 .

Table 3.2: Charge Accumulated 

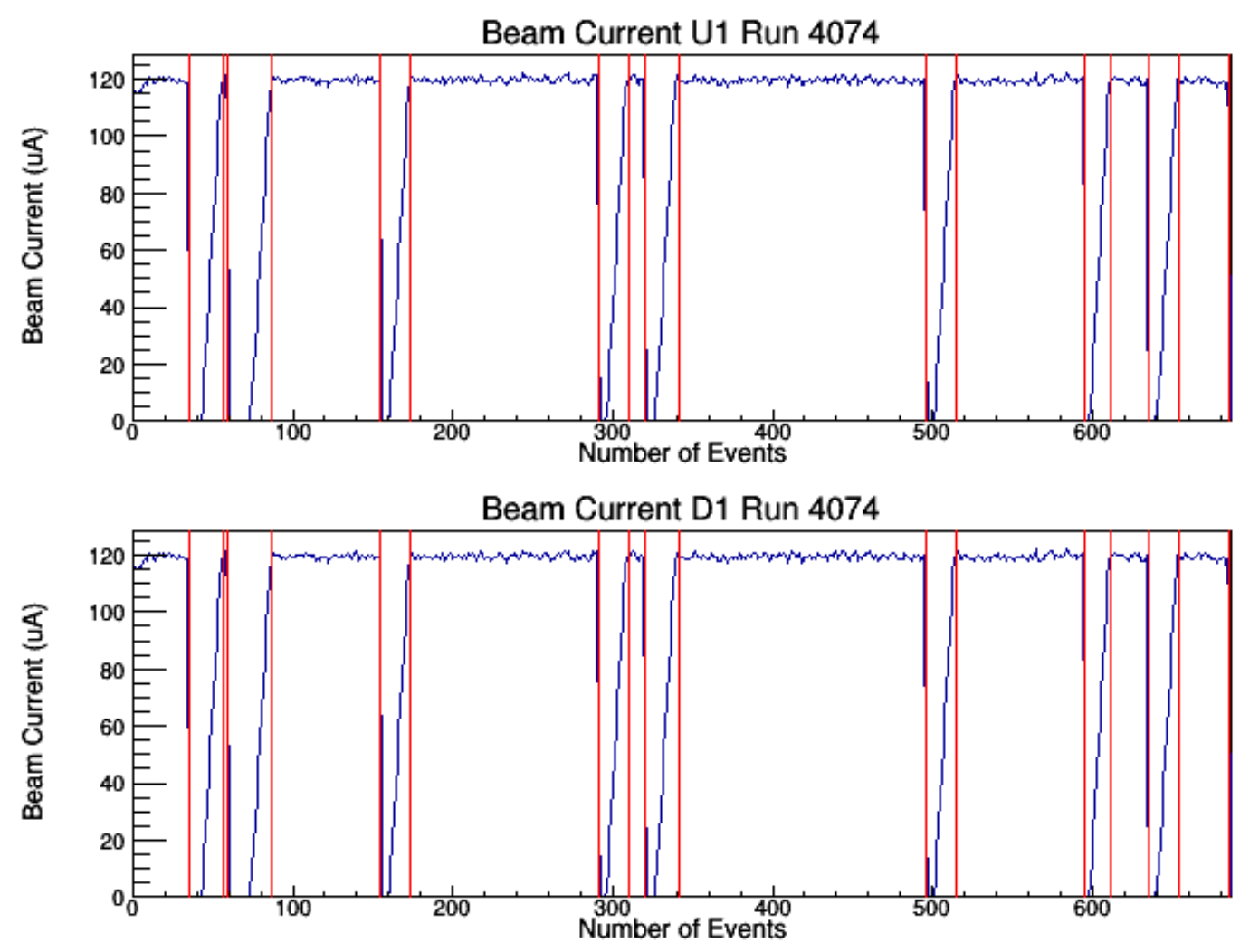

Figure 3.1: BCM Readouts for Run 4074. These plots show the $U_{1}$ and $D_{1}$ BCM measurements for run 4074. The cuts are applied two scaler readouts before (after) the BCMs register $90 \%$ of the $120 \mu \mathrm{A}$ operating current for the falling (rising) edge.

\subsection{Corrections and Efficiencies}

\subsubsection{Live-time Correction}

Now we have measured how many total electrons are incident on our target. However, we have not yet accounted for the electronic dead-time discussed in Section 2.8 and Equation 2.10. While the electronics discard some valid trigger events because the system is busy processing the previous event scalers still record every trigger created regardless of if it is recorded by the main DAQ. This means that the live-time of the system can be calculated by taking the ratio of the total triggers recorded by the DAQ electronics to the total hardware triggers recorded by the scalers.

Table 3.3 contains the live-time for each run as well as the weighted average of 
live-times for the cumulative runs. The discrepancy between the live-times for the first and last three runs is due to the differing event rates on the right arm during each of these sets of runs. When the right arm has a higher data rate the live-time for both arms decreases. The average live-time of $95.27 \%$ is then applied as a correction of $\frac{1}{0.9527}$ to the total number of elastic electrons detected.

\begin{tabular}{|cccc|}
\hline Run & $\begin{array}{c}\text { Hardware } \\
\boldsymbol{T}_{\mathbf{3}}\end{array}$ & $\begin{array}{c}\text { Electronic } \\
\boldsymbol{T}_{\mathbf{3}}\end{array}$ & Live-time \\
\hline 3892 & 49802 & 42982 & 0.86306 \\
3893 & 485367 & 427476 & 0.88073 \\
3894 & - & 311724 & $0.87912^{*}$ \\
4073 & 106003 & 103741 & 0.97866 \\
4074 & 1124275 & 1102321 & 0.98047 \\
4075 & 1152972 & 1129955 & 0.98004 \\
\hline Weighted & & & $\mathbf{0 . 9 5 2 7}$ \\
Average & & & \\
\hline
\end{tabular}

Table 3.3: Live-time per Run. Note that run 3894 had no End of Run readout so the live-time is a weighted average of the two runs, 3892 and 3893, taken the same day.

\subsubsection{Trigger Efficiency}

The main trigger, $\mathrm{T}_{3}$, for E08-014 was a coincidence of $\mathrm{S}_{1}, \mathrm{~S}_{2 m}$, and the GC detectors. However, this trigger is not perfectly efficient. To measure $\mathrm{T}_{3}$ 's efficiency we use trigger $\mathrm{T}_{4}$, which is the coincidence of one of either $\mathrm{S}_{1}$ or $\mathrm{S}_{2 m}$ and the GC, and Equation 3.5. Here $P S_{3,4}$ are the prescale values of $\mathrm{T}_{3}$ or $\mathrm{T}_{4}$, and $n_{T_{3,4}}$ are the number of triggers of either $\mathrm{T}_{3}$ or $\mathrm{T}_{4}$. Figure 3.2 shows the $\mathrm{T}_{3}$ efficiencies for each of the runs, and Table 3.4 lists these efficiencies along with the weighted average of the runs.

$$
T_{3_{e f f}}=\frac{P S_{T_{3}} \times n_{T_{3}}}{P S_{T_{3}} \times n_{T_{3}}+P S_{T_{4}} \times n_{T_{4}}}
$$

\subsubsection{VDC Single Track Efficiency}

In general the VDCs are very efficient, but it is possible for particles to make several tracks in the VDCs. This can cause issues with track reconstruction. For the 


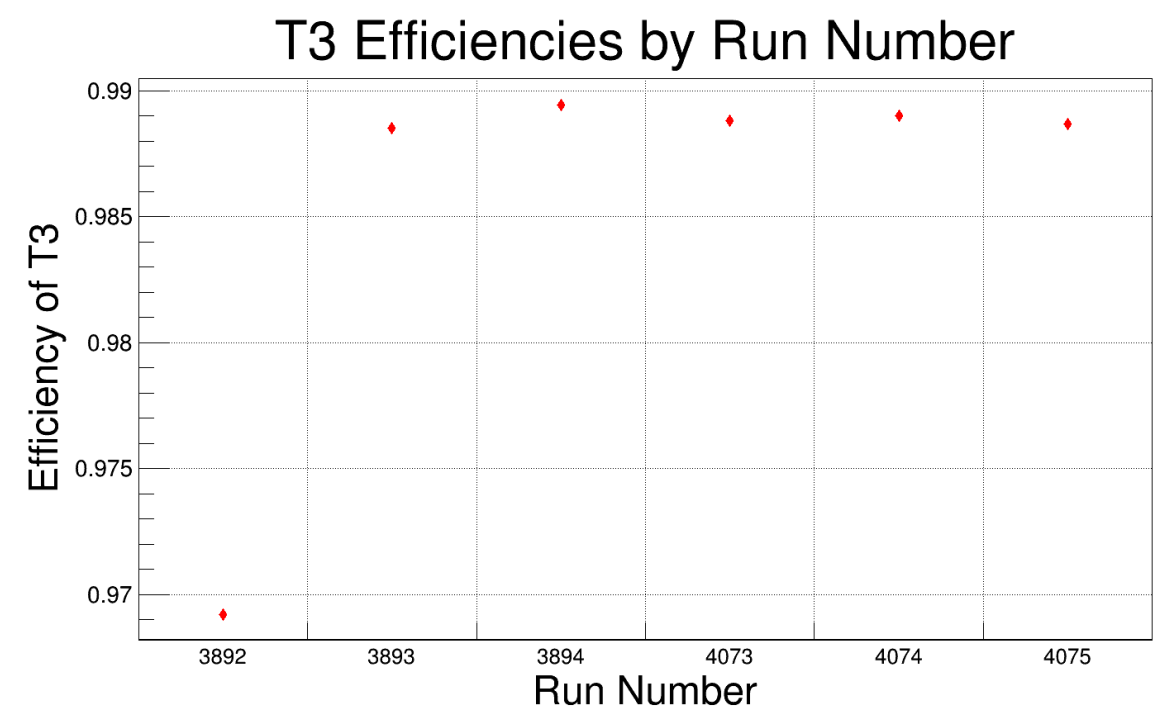

Figure 3.2: T3 Efficiency by Run.

\begin{tabular}{|cc|}
\hline Run & $\boldsymbol{T}_{\mathbf{3}}$ Efficiency \\
\hline 3892 & 0.9692 \\
3893 & 0.9885 \\
3894 & 0.9894 \\
4073 & 0.9888 \\
4074 & 0.9890 \\
4075 & 0.9887 \\
\hline Weighted & $\mathbf{0 . 9 8 8 6}$ \\
Average & \\
\hline
\end{tabular}

Table 3.4: $\boldsymbol{T}_{\mathbf{3}}$ Efficiency

analysis of E08-014 only events making a single track in the VDCs that also passed PID cuts were analyzed. This single track efficiency can be calculated with equation 3.6. Here the variable $N_{\text {track }=1}$ is the number of events making only a single track, and the variable $N_{0 \leq t r a c k \leq 4}$ is the number of events producing between one and four tracks. For the LHRS during experiment E08-014 $\epsilon_{V D C}$ was found to be $99.175 \%$, and Table 3.5 gives the breakdown for both detectors by number of tracks seen. This introduces a small correction factor of $\frac{1}{0.99175}$ to the electron yield. For more detailed information on this calibration see [7] where these values were calculated.

$$
\epsilon_{V D C}=\frac{N_{t r a c k=1}}{N_{0 \leq t r a c k \leq 4}}
$$




\begin{tabular}{|cccccc|}
\hline $\boldsymbol{N}_{\text {track }}$ & $\mathbf{0}$ & $\mathbf{1}$ & $\mathbf{2}$ & $\mathbf{3}$ & $\mathbf{4}$ \\
\hline HRSL & $0.030 \%$ & $99.175 \%$ & $0.743 \%$ & $0.045 \%$ & $0.005 \%$ \\
HRSR & $0.048 \%$ & $99.360 \%$ & $0.545 \%$ & $0.039 \%$ & $0.007 \%$ \\
\hline
\end{tabular}

Table 3.5: VDC Track Efficiency for HRSs

\subsubsection{Particle Identification}

When a trigger is seen it is important to be able to identify what particle caused the trigger. For this analysis we are only interested in electrons, but pions occasionally cause triggers as well. Figure 3.3 shows a plot of the two pion rejector calorimeters used for PID in E08-014. The data in this figure is not the data used in this analysis, and the figure is only intended to show where we expect to see certain particles in this thesis' analysis.

As discussed in Section 2.7.4 electrons will leave large signals in the PRs, and pions much smaller signals. Using this property of the PRs we can show the area almost certain to be electrons shaded in blue, and the area we expect to be pions is shaded in red. Note that the blue (red) region is not all of the electrons (pions), but triggers in the blue (red) region are almost certainly electrons (pions). (Note that the shaded regions are not cuts placed on the final data. They are only used to check the pion contamination of our sample.) The other low energy events not identified as pions are generally $\delta$, or knock-on, electrons. These events are created when particles strike the metals of the detector frames and windows and knock out an electron. On rare occasions these electrons have sufficient energy to create a shower in the PRs. We can see for the run shown in Figure 3.3 that there is a non-zero pion contamination in the sample.

Alternatively, we can look at the same data in the GC to identify the particles as shown in Figure 3.4 (again this data is an example and was not used in this analysis). We discussed how the gas in the GC is chosen for electrons to emit Cherenkov radiation in Section 2.7.3. Due to this gas choice pions rarely create Cherenkov radiation in the GC. We can see this difference in Figure 3.4 by identifying the bulk of the signal, blue, 


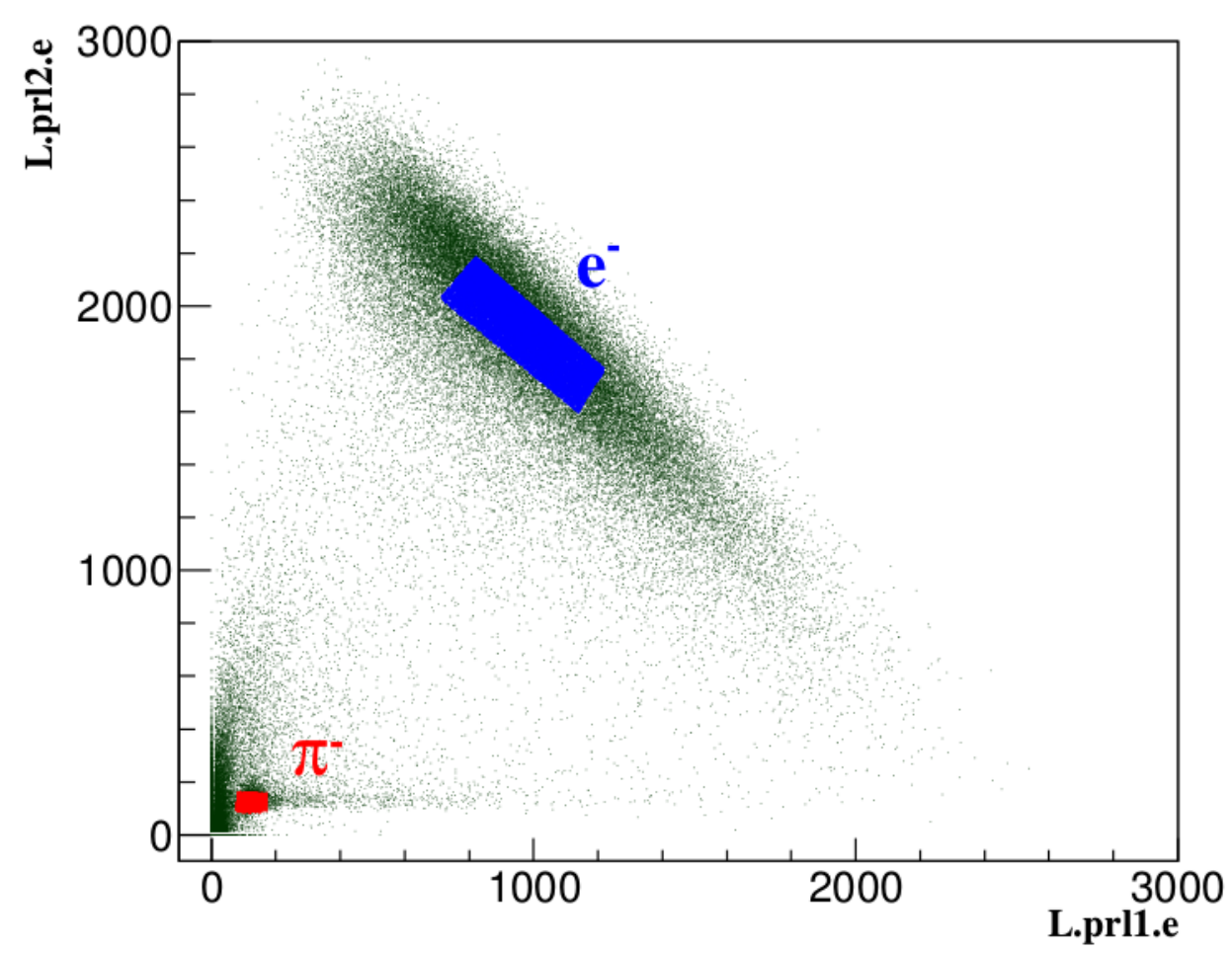

(a) Pion Rejectors

Figure 3.3: PID with the Pion Rejectors. This plot does not show the data used in this analysis. It is intended only to show where we expect to see certain particles in this thesis' analysis. The $X$-axis represents pion-rejector 1 , and the $Y$-axis represents pion-rejector 2. Both axes are in units of ADC channel. Pions are found in the lower energy ADC channels. The red box indicates an area in which the particles are almost certainly pions, but the red box is not a cut applied to the data. The electrons are found in the higher energy ADC channels. The blue box indicates an area in which the particles are almost certainly electrons, but the blue box is not a cut applied to the data. The boxed regions were only used to check the pion contamination. Image from 7 .

as electrons, and the lowest signals, red, as pions. Again, the blue (red) region is not all of the electrons (pions), but triggers in the blue (red) region are almost certainly electrons (pions) (again these are not cuts on the final data and are only used to check the pion contamination). In this data we see the non-zero pion contamination again. Fortunately, as with the PR plots, the pions and electrons are easy to distinguish from one another. 


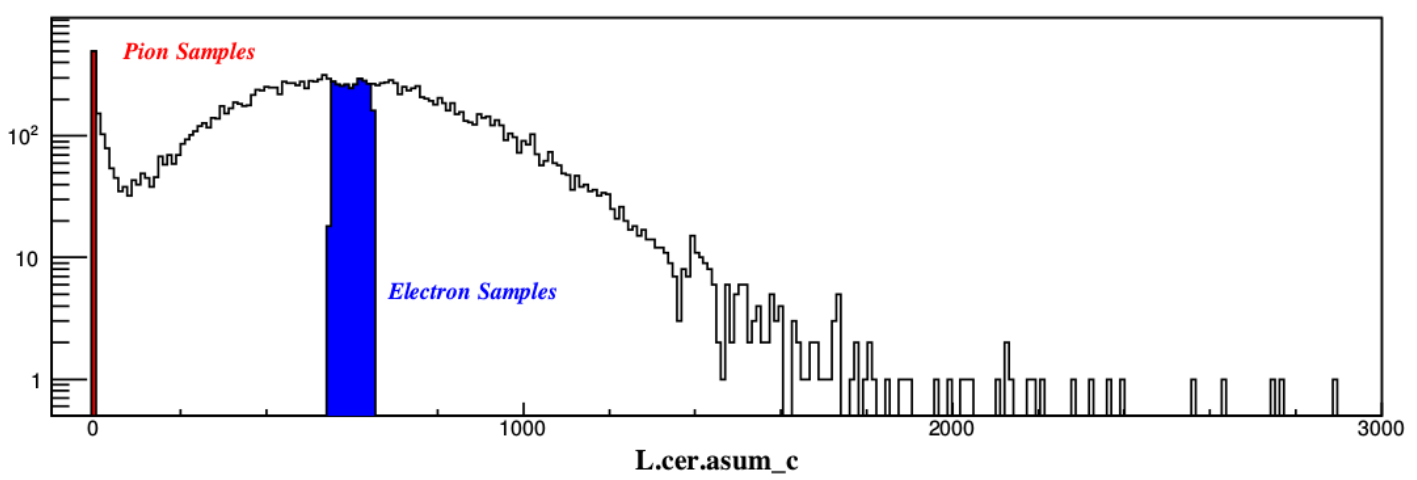

Figure 3.4: PID with the Gas Cherenkov. This plot does not show the data used in this analysis. It is intended only to show where we expect to see certain particles in this thesis' analysis. The $X$-axis is in units of ADC channel, and the $Y$-axis is the number of events detected. Pions are found in the lower energy ADC channels. The red area shows where almost all of the particles should be pions, but the red area is not a cut applied to the data. Electrons are found in the bulk of the distribution between ADC channels 100 and 1400. The blue area shows where almost all of the particles should be electrons, but the blue area is not a cut applied to the data. The shaded areas were only used to check the pion contamination. Image from 7 .

Now let us examine the combined six experimental runs used in this analysis using the PRs and GC. Figure 3.5 shows the PRs for our data with reasonable physics cuts applied to the data. We see the same 'cloud' of electrons in the higher PR ADC channels indicating that electron energy is being well measured by the PRs. The red box in this cloud shows a similar region to before where we are almost certain to have 'good' electrons. However, when we search for the pion cloud in the lower energy channels, represented by the small red box in the lower left, we see very few pions at all. This is a result of Kin 3.2 producing few pions that can be measured by the detectors so our pion contamination is extremely low. The small number of pions can be eliminated by placing a diagonal cut on the data represented by the green line in Figure 3.5. Note that the only cut actually applied to the data was the diagonal green line. The red boxes were only used to check the pion contamination and were not cuts applied to the final data.

We can also examine the GC, Figure 3.6, as we did previously. Once again we have applied reasonable physics cuts to the data. We see a large bulk signal at its strongest 


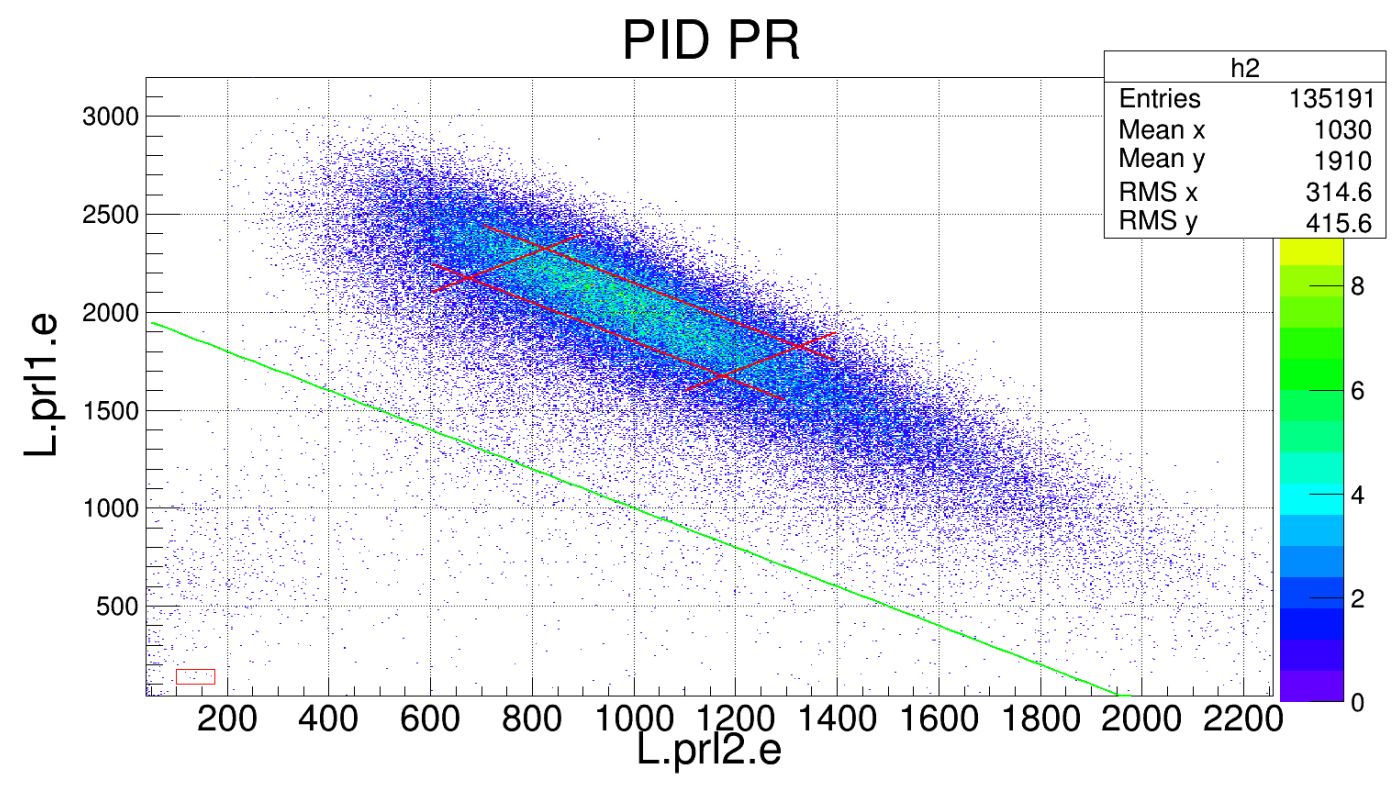

Figure 3.5: PID with the Pion Rejectors. This plot shows the six runs used in this analysis. The $X$-axis represents pion-rejector 2 , and the $Y$-axis represents pion-rejector 1. Both axes are in units of ADC channel. The red box in the low energy ADC channels shows a region in which pions would be located if they were in the data. Few events are located in this pion region indicating few pions are contaminating the sample. The red box in the cloud of events in higher ADC channels indicates the region in which events should definitely be electrons. These boxes were only used to check the pion contamination but are not cuts applied to the data. The green line is a cut applied to the final data. This cut removes junk events, $\delta$-electrons, and any of the few pions in the sample below the green line.

between channels 300 and 500, shown between two red lines, as expected. This shows that we are detecting electrons well with the GC. Looking in the lower ADC channels for pions we again see that there seem to be very few in our data. In fact, below ADC channel 80 we see only 15 events showing that there are very few pions in our sample. As a result when making the final cuts to the data only the diagonal cut on the PRs was used to eliminate possible pions, and more likely, junk electrons which are most probably $\delta$-electrons. 


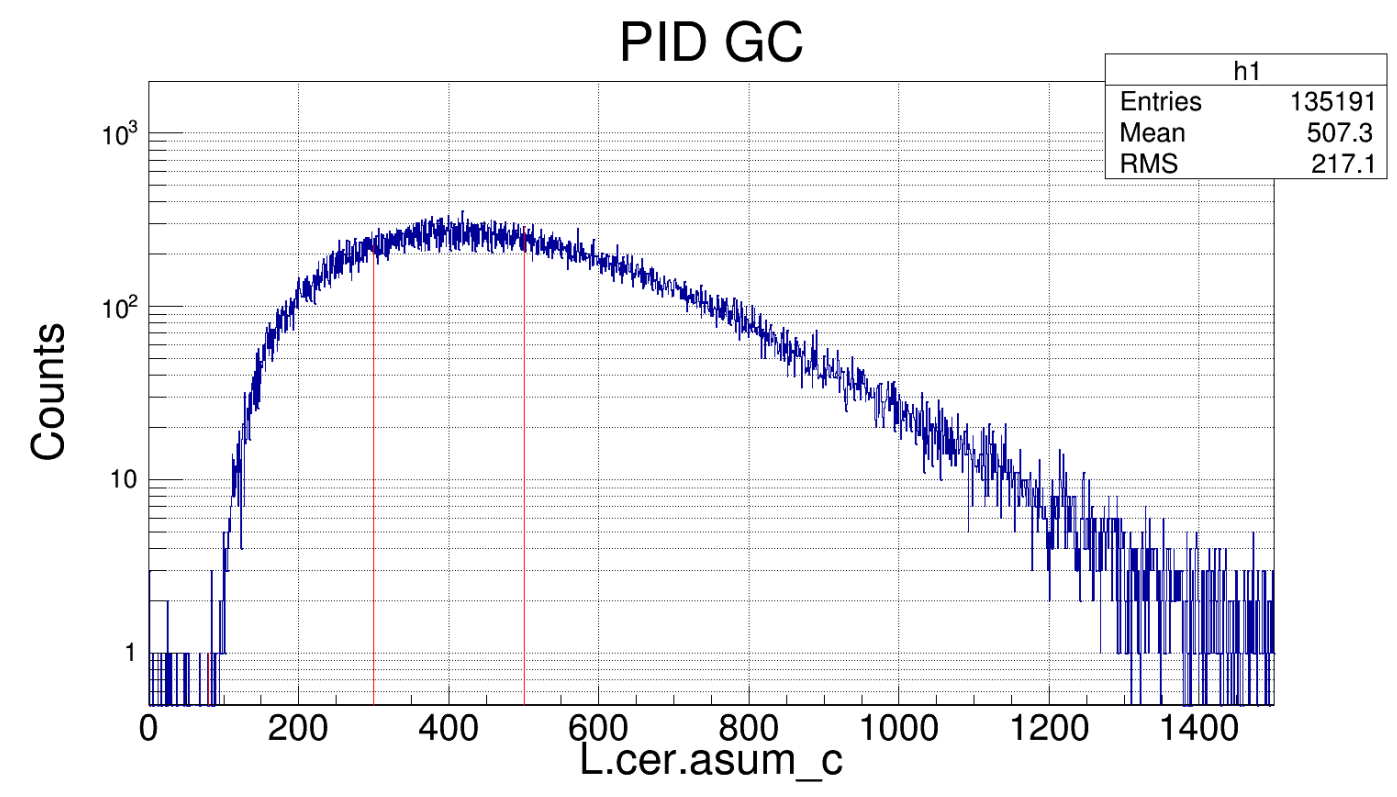

Figure 3.6: PID with the Gas Cherenkov. This plot shows the GC ADC for the six runs used in this analysis. The $X$-axis is in units of ADC channel, and the $Y$-axis is the number of events detected. Pions would be found in the lower energy ADC channels below the red line at channel 80 , but that region contains almost no events so there are very few pions in the sample. The area between the red lines at channels 300 and 500 shows where almost all of the particles should be electrons. The two areas where we expect all events to be pions or electrons were only used to check the pion contamination, but they are not applied as cuts to the data.

\subsubsection{Gas Cherenkov Efficiency}

Now that we have demonstrated that very few pions are contaminating our electron sample we must still account for inefficiencies in the GC. During E08-014 one of the ten PMTs in the LHRS GC was slightly inefficient, and this PMT happens to measure electrons with the kinematics of those elastically scattered from ${ }^{3} \mathrm{He}$ at this analysis' kinematics. To study the GC efficiency we can use $\mathrm{T}_{3}$, the main trigger, which is a coincidence of $\mathrm{S}_{1}, \mathrm{~S}_{2 m}$, and the GC detectors as well as $\mathrm{T}_{7}$, which is a coincidence of $\mathrm{S}_{1}$ and $\mathrm{S}_{2 m}$. The number of events firing both $\mathrm{T}_{3}$ and $\mathrm{T}_{7}, \mathrm{~T}_{3 \& 7}$, divided by $\mathrm{T}_{7}$ will yield the GC efficiency for these E08-014 runs as shown in Equation 3.7 21.

$$
\epsilon_{G C}=\frac{T_{3 \& 7}}{T_{7}}
$$


Before we find the GC efficiency let us first place some reasonable kinematic and acceptance cuts on the combined data of our six experimental runs (see Section 3.6 for details on the cut values). Figure 3.7 shows a plot of $T_{7}$ on the left and $T_{3 \& 7}$ on the right with physics cuts. These cuts are fairly strict and include a cut on $x_{B j}$ to isolate the elastic peak so there are few events. The events form the characteristic elastic band shape across the GC PMTs. Taking the ratio we find that $\epsilon_{G C}=196 / 203=0.966$. This is still fairly efficient accounting for the slightly inefficient PMT. Knowing the GC inefficiency allows us to scale the electron yield by 203/196 to correct for this inefficiency.
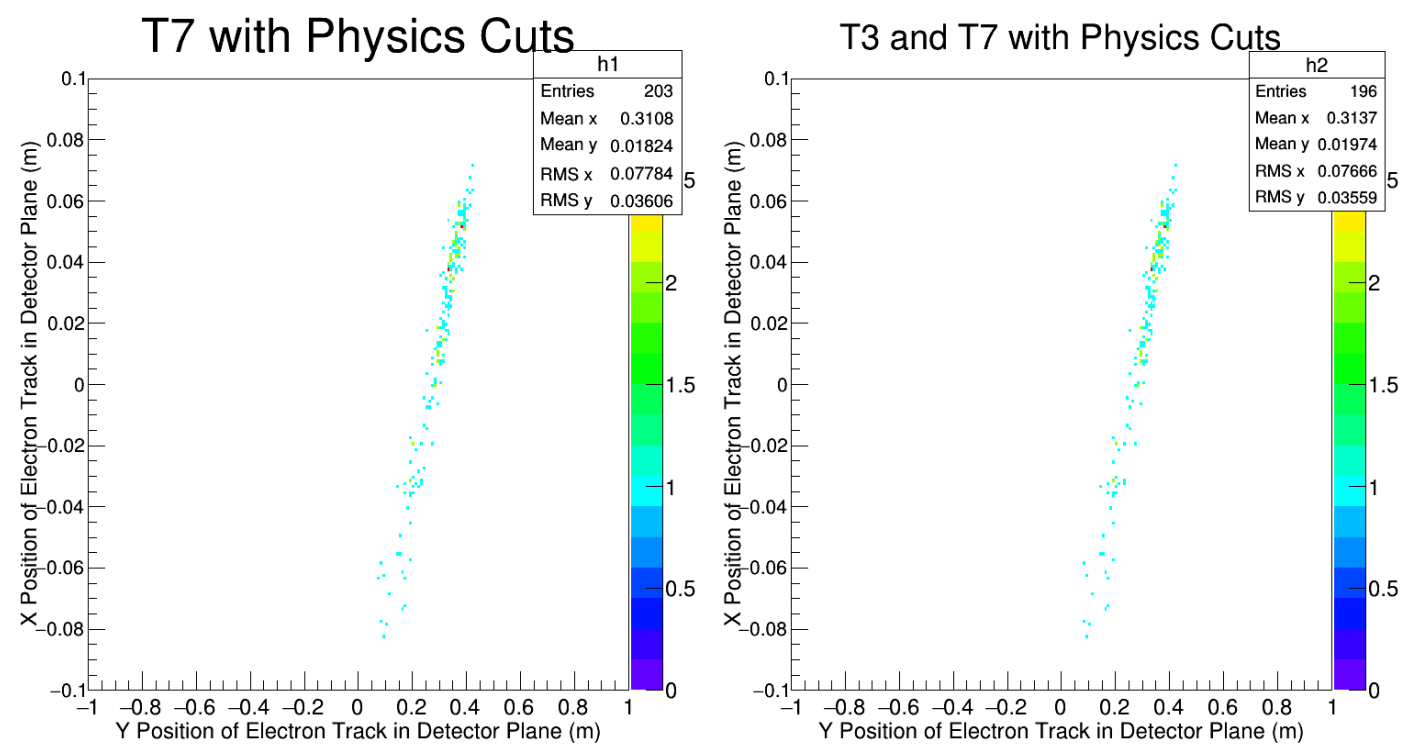

Figure 3.7: GC Efficiency. The left plot is $T_{7}$ with physics cuts and the right is $T_{3 \& 7}$ with physics cuts.

\subsection{Target Density}

As the electron beam passes through the gaseous ${ }^{3} \mathrm{He}$ target it causes the target to heat considerably. In the ideal case the target would heat uniformly. However, studies of the target boiling effect during E08-014 indicate that the strength of the target boiling effect was correlated to the location along the target's long axis, $Z_{\text {react }}$. Note 
that the term 'boiling' is not used to indicate the physical process of liquid boiling to gas as the ${ }^{3} \mathrm{He}$ target was already gaseous. Instead, boiling is meant to indicate a change in gas density in the target due to temperature differences. Figure 3.8 shows events detected along $Z_{\text {react }}$ for various beam currents. The two peaks at either end of the plot are the aluminum endcaps of the target. It is clear from this plot that the density of the gas across the cell is not constant otherwise the event rate would be approximately constant. The bump in density from $-8 \mathrm{~cm}$ to $-3 \mathrm{~cm}$ is due to this part of the cell being more effectively cooled by the cryosystem leading to a higher gas density. For a detailed discussion of the target boiling studies see [7] Section 5.4.1 and Appendix D. Ignoring the large bump, the small downward slope of the density observed is due to the fact that the scattering angle seen by the spectrometer changes slightly along the length of the cell leading to a change in $Q^{2}$.

The target boiling effects were the result of using a significantly higher beam current than the ${ }^{3} \mathrm{He}$ cells were designed to cool [22]. (This higher current did provide the silver lining of helping to create enough elastic electrons for this analysis to be possible.) To better understand the density of the target when the beam was on Silviu Covrig created a computer simulation of the target under the experimental conditions. The computational fluid dynamics (CFD) simulation studied the behavior of ${ }^{4} \mathrm{He}$ based on its density, specific heat, thermal conductivity, and viscosity. The simulation also factored in the conditions of the cell such as pressure and temperature which were measured during the experiment [23]. The mechanics of the CFD simulation are best described in Covrig's own words,

"This method of calculation is called finite volume element, which means that the volume of the target cell is broken into smaller volumes, a process controlled by the size of the mesh. Fluid dynamics equations, transport equations or equations for any scalar/vector of interest are solved on these elements of volume or computational cells and predictions are made for the fields of temperature, velocity, density etc. in the whole volume of the 


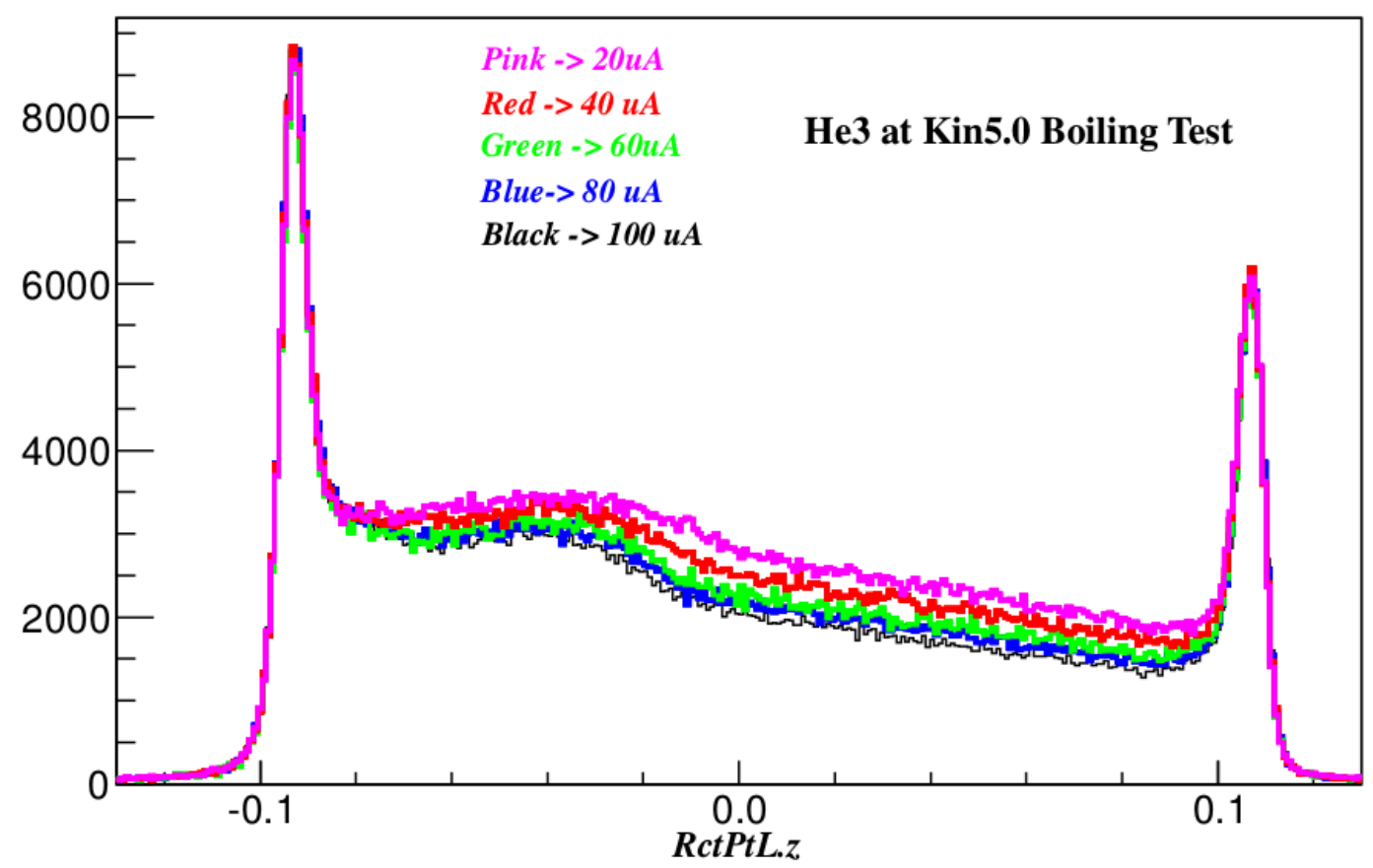

Figure 3.8: ${ }^{3}$ He Boiling Effect. The $X$-axis here represents $Z_{\text {react }}$ (direction along the cell's long axis) and is in units of meters. The $Y$-axis shows the number of events. The two peaks at either end of the distribution come from the endcaps of the target and are cut from the final sample. The different colored lines represent different beam currents. If the target density was constant throughout the cell the lines between the endcaps would be flat. However, there is clearly a bump around $Z_{\text {react }}=-0.02$ $\mathrm{m}$. Above this value the number of events decreases due to the density of the ${ }^{3} \mathrm{He}$ gas decreasing. This decreased density occurs because the cell was unevenly cooled and gas further from the coolant heated decreasing the gas density. Ignoring the large bump, the small downward slope of the density observed is due to the fact that the scattering angle seen by the spectrometer changes slightly along the length of the cell leading to a change in $Q^{2}$. Image from 7].

geometry. At any given $z$-location along the beam line the raster area is, say, $9 \mathrm{~mm}$ by $2 \mathrm{~mm}$ or $3 \mathrm{~mm}$ by $3 \mathrm{~mm}$. If the mesh size was, say, 0.25 $\mathrm{mm}$, then you could expect about $12 \times 12$ or 144 volumetric cells at that $z$-location. For each of these cells the program predicts the velocity of the fluid, its density, temperature etc. at the center of the cell. If you then make a $2 \mathrm{D}$ plot of density vs. beam line $z$-location, the program plots a vertical dotted line that represents the spread of density among the volumetric cells at that $z$-location. The spread in value is given by 
what happens at that $z$-locations over the area of the raster. The fluid may not have constant velocity in the raster area at that $z$-location, so heating from the beam will decrease more or less its density in volumetric cells that move slower or faster respectively [23]."

This target density study was performed on ${ }^{4} \mathrm{He}$ so the results of the study need to be related to ${ }^{3} \mathrm{He}$. This can be done by using the isotopic nature of ${ }^{3} \mathrm{He}$ and ${ }^{4} \mathrm{He}$ as well as the ideal gas law. We can begin by using the relation between the fractional change in density between ${ }^{3} \mathrm{He}$ and ${ }^{4} \mathrm{He}$ as given in Equation 3.8, where $d \rho_{\left({ }^{3} \mathrm{He}\right)}\left(d \rho_{\left({ }^{4} \mathrm{He}\right)}\right)$ is the fractional change in density for ${ }^{3} \mathrm{He}\left({ }^{4} \mathrm{He}\right), A_{3}\left(A_{4}\right)$ is the number of nucleons for ${ }^{3} \mathrm{He}\left({ }^{4} \mathrm{He}\right), P_{3}\left(P_{4}\right)$ is the pressure for ${ }^{3} \mathrm{He}\left({ }^{4} \mathrm{He}\right)$, and $I_{3}\left(I_{4}\right)$ is the beam current for ${ }^{3} \mathrm{He}\left({ }^{4} \mathrm{He}\right)$ 23]. $d \rho$ is defined as $\frac{\rho-\rho_{0}}{\rho_{0}}$, where $\rho$ is the density with the beam on and $\rho_{0}$ is the density with the beam off [23].

$$
d \rho_{\left({ }^{3} \mathrm{He}\right)}=\frac{A_{4}}{A_{3}} \frac{P_{4}}{P_{3}} \frac{I_{3}}{I_{4}} d \rho_{\left({ }^{4} \mathrm{He}\right)}
$$

Plugging in the corresponding values from the experimental setup and the simulation we can rewrite the product in Equation 3.8 as Equation 3.9 .

$$
R_{1}=\frac{A_{4}}{A_{3}} \frac{P_{4}}{P_{3}} \frac{I_{3}}{I_{4}}=\frac{4}{3}
$$

We can then use the ideal gas law to relate the densities of ${ }^{3} \mathrm{He}$ and ${ }^{4} \mathrm{He}$ without beam as in Equation 3.10 .

$$
\rho_{0\left({ }^{3} \mathrm{He}\right)}=\frac{A_{3}}{A_{4}} \frac{P_{3}}{P_{4}} \frac{T_{4}}{T_{3}} \rho_{0\left({ }^{4} \mathrm{He}\right)}
$$

Once again we can rewrite the product in Equation 3.10 using the experimental and simulation conditions as Equation 3.11.

$$
R_{2}=\frac{A_{3}}{A_{4}} \frac{P_{3}}{P_{4}} \frac{T_{4}}{T_{3}}=0.745
$$

Finally we can solve Equation 3.8 for $\rho_{\left({ }^{3} \mathrm{He}\right)}$ by plugging in Equation 3.10, This 
gives us the equation describing the ${ }^{3} \mathrm{He}$ density with the beam on as shown in Equation 3.12 .

$$
\rho_{\left({ }^{3} \mathrm{He}\right)}=R_{1} R_{2} \rho_{\left({ }^{4} \mathrm{He}\right)}+\rho_{0\left({ }^{3} \mathrm{He}\right)}\left(1-R_{2}\right)
$$

We can then get the ${ }^{3} \mathrm{He}$ density along the $Z$-axis of the target from the ${ }^{4} \mathrm{He}$ CFD simulation results. By integrating over the $Z$-axis we find the average absolute density of the target. This density was found to be $0.013 \mathrm{~g} / \mathrm{cm}^{3} \pm 0.0004 \mathrm{~g} / \mathrm{cm}^{3}$. For a more in depth discussion of this density extraction see [23].

\subsection{Cuts}

Not all of the electrons we detect necessarily come from regions we are interested in studying. For example, any electrons that originated outside of the target or that have a momentum greatly different than the momentum setting of the spectrometer are not wanted in our electron sample. To ensure we are studying the electrons scattering from the target we impose a series of physics cuts for the spectrometer's acceptance as well as some of the kinematics. In Section 3.4 .4 we already discussed a cut to remove the very small number of pions as well as the $\delta$-electrons from our sample. In this section will discuss the other major cuts we have imposed on the data which are summarized in Table 3.6 .

\begin{tabular}{|r|ll|}
\hline Cut Type & Minimum & Maximum \\
\hline$Y$ Target & $-0.03 \mathrm{~m}$ & $0.028 \mathrm{~m}$ \\
$\theta$ & $-0.049 \mathrm{rad}$ & $0.042 \mathrm{rad}$ \\
$\phi$ & $-0.03 \mathrm{rad}$ & $0.03 \mathrm{rad}$ \\
$d P=\frac{P-P_{0}}{P_{0}}$ & -0.02 & 0.03 \\
\hline
\end{tabular}

Table 3.6: Summary of Acceptance Cuts

Each of these cuts was made using several techniques. Let us take the $Y_{\text {target }}$ $\left(Y_{\text {target }}=\sin \left(\theta_{H R S}\right) Z_{\text {react }}\right)$ cut as our example. The first technique is a visual assessment of the range of data that we wish to accept. Figure 3.9 shows the $Y_{\text {target }}$ 
plot of the experimental data, and the red region shows the range of data we chose to cut on. Notice the two peaks at either end of the data. These peaks are created by the aluminum endcaps, and as such are not of interest in this analysis. To remove these peaks from the sample two cuts were placed on $Y_{\text {target }}$.

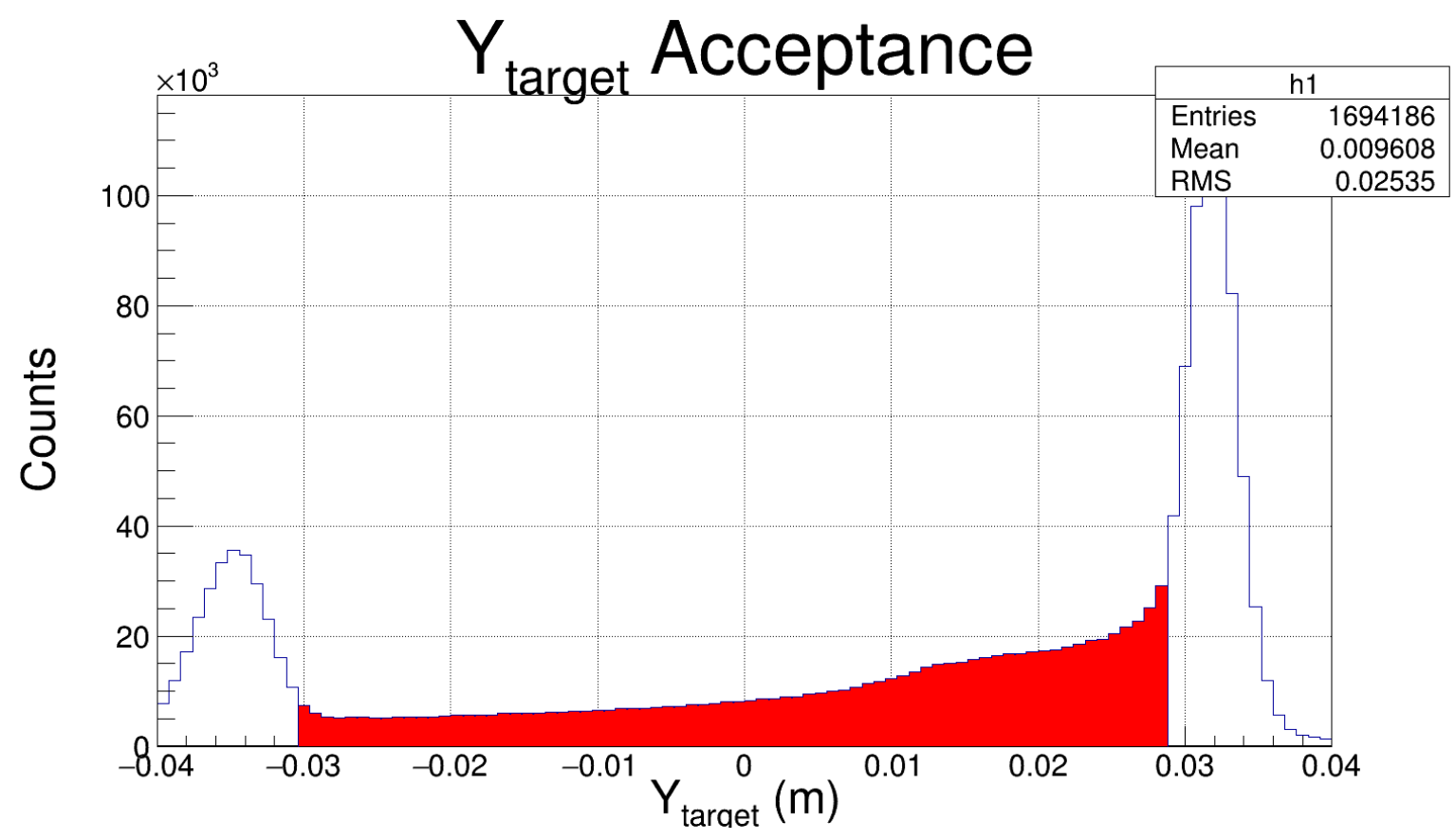

Figure 3.9: $\boldsymbol{Y}_{\text {target }}$ Acceptance. The red acceptance region runs from $-0.03 \mathrm{~m}$ to $0.028 \mathrm{~m}$. The peaks at either end of the distribution are from the target's endcaps and were cut from the sample. The bump starting around $0.01 \mathrm{~m}$ is due to the increased target density due to the boiling effects discussed in Section 3.5 .

The second technique involves intentionally using cuts that are too wide, and then continually making the cuts tighter until the cross section result no longer changes as the cuts are made tighter. When the cuts are too wide regions without the ${ }^{3} \mathrm{He}$ gas and/or outside of our kinematic region of interest are accepted giving a cross section value that is dependent on the placements of the cuts. Once the cuts are strict enough to capture only the electrons elastically scattered from ${ }^{3} \mathrm{He}$ the cross section becomes stable. Cuts are made such that they accept the maximum number of electrons in the region where the cross section is stable independent of the cuts. Using both techniques the acceptance region for $Y_{\text {target }}$ was chosen as the region between $-0.03 \mathrm{~m}$ to $0.028 \mathrm{~m}$. 
Now let us look at the remaining cuts for $\theta, \phi$, and $d P$ (the momentum fraction $d P$ is often also referred to as $\delta$ ). Figure 3.10 shows a plot of $\theta$ (the out-of-plane angle) for experiment E08-014. This cut was primarily made visually and runs from -0.042 to 0.049 radians. Figure 3.11 shows a plot of $\phi$ (the in-plane angle) for experiment E08-014. This cut was made both visually and with the second method of reducing the cut width until the cross section value becomes stable. The second method is more important for $\phi$ than for $\theta$ since the cross section is more sensitive to $\phi$. The cut for $\phi$ runs from -0.03 to 0.03 radians. Finally, Figure 3.12 shows a plot of $d P$ for experiment E08-014. This cut was made mostly using the second method of checking for cross section stability. The $d P$ cut runs from -0.02 to 0.03 , where $d P=\frac{P-P_{0}}{P_{0}}, P$ is the electron momentum, and $P_{0}$ is the spectrometer's momentum setting.

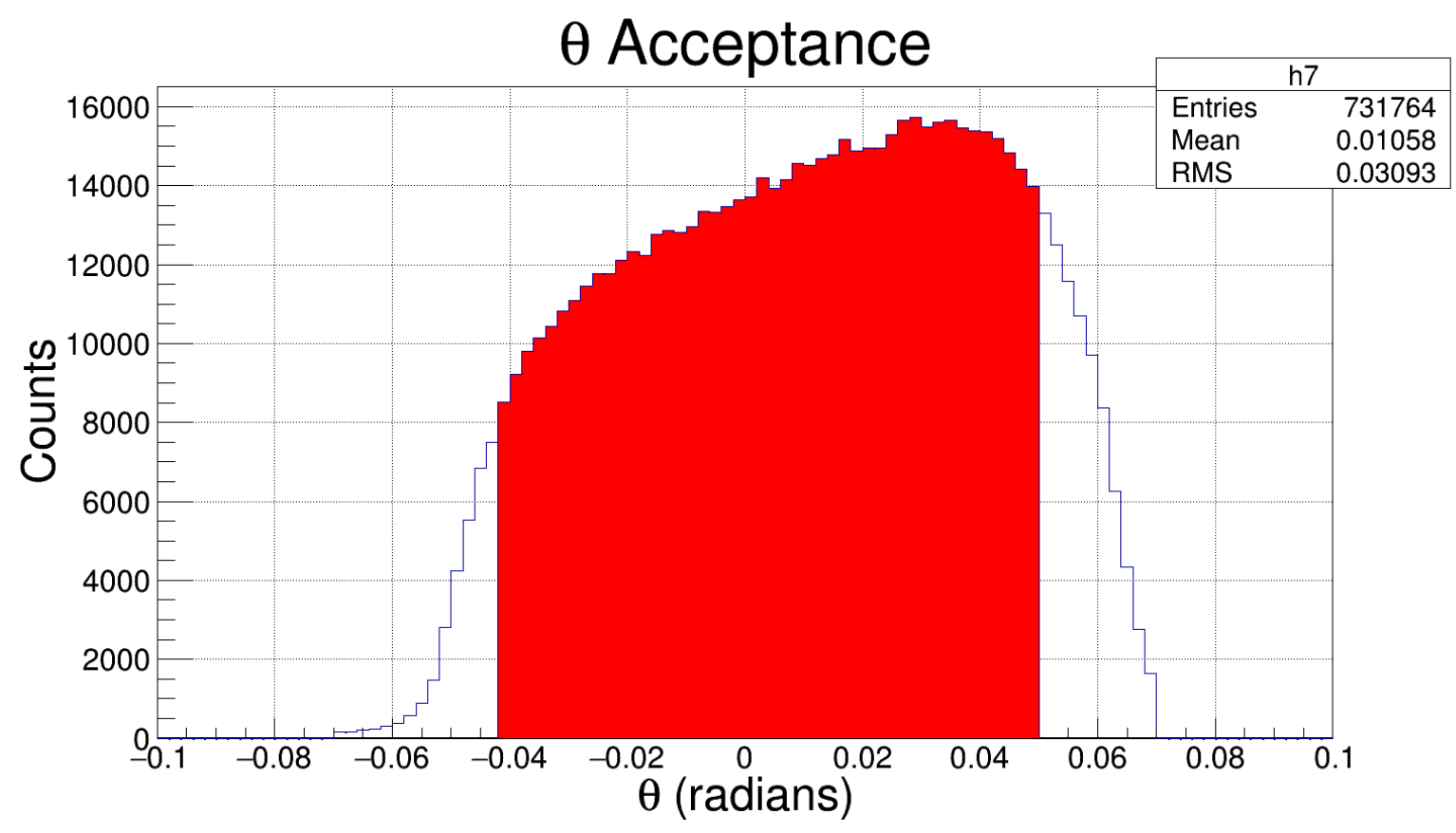

Figure 3.10: $\boldsymbol{\theta}$ Acceptance. $\theta$ is the out-of-plane angle. The red acceptance region runs from -0.042 radians to 0.049 radians. 


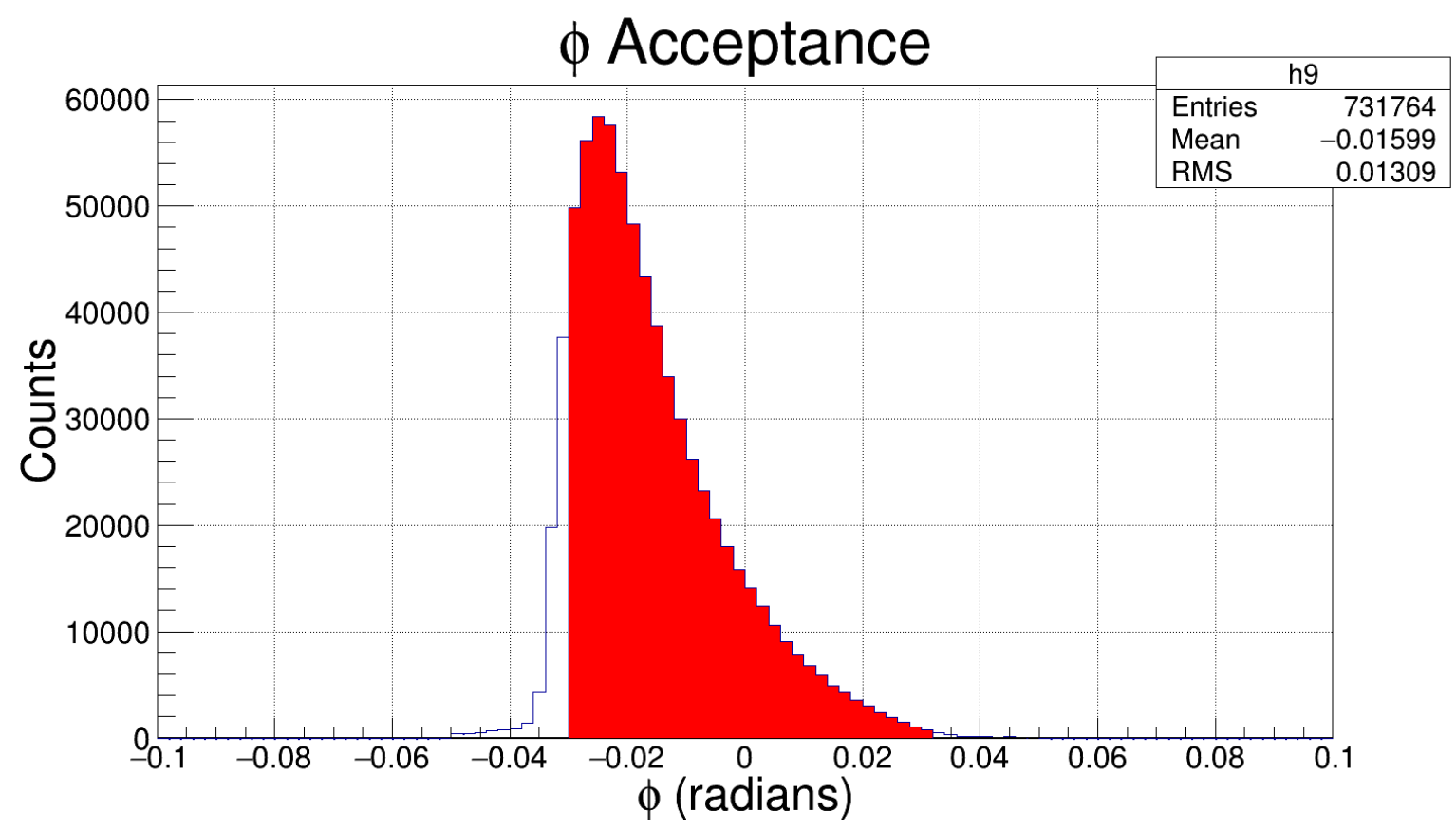

Figure 3.11: $\phi$ Acceptance. $\phi$ is the in-plane angle. The red acceptance region runs from -0.03 radians to 0.03 radians.

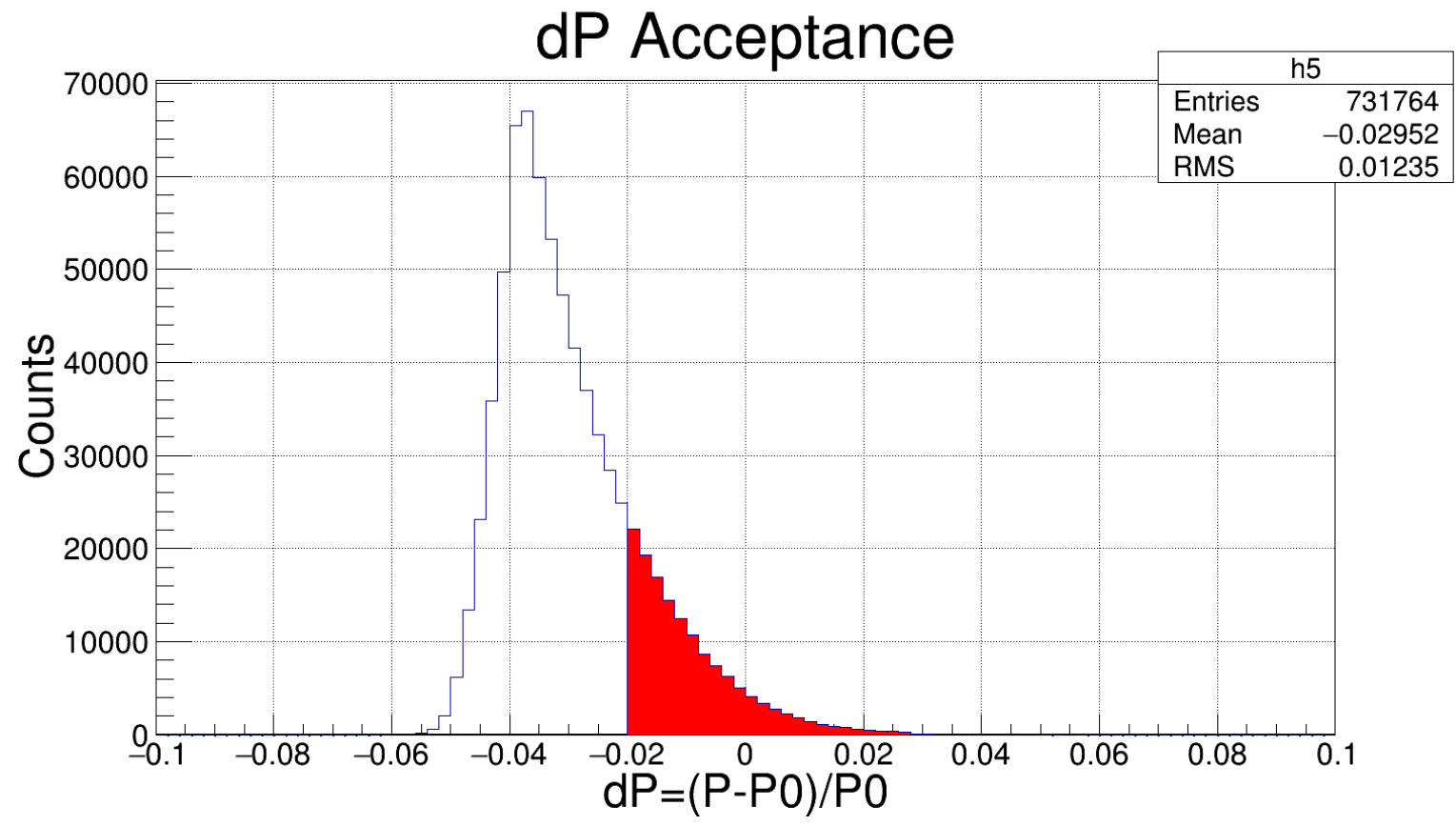

Figure 3.12: $\boldsymbol{d P}$ Acceptance. $d P$ is the momentum fraction. The red acceptance region runs from -0.02 to 0.03 . 


\section{7 $\quad{ }^{3}$ He Elastic Cross Section Monte Carlo}

To extract the final ${ }^{3} \mathrm{He}$ elastic cross section value for this analysis a physics simulation Monte Carlo program called SIMC was used. This program is primarily used by JLab's Halls A and C to simulate electron scattering experiments. SIMC contains the geometry of the Hall A spectrometers including their various apertures and the materials that comprise them. SIMC uses an event generator to create electrons which then scatter from a given target. These events are transported through the spectrometer based on an optics matrix which allows for the transformation of detector coordinates to target coordinates. SIMC then records the final states of the scattered electrons as if they were viewed by a detector.

SIMC requires numerous inputs before generating the initial events. The program must be given ranges in momentum, in-plane angle $(\phi)$, out-of-plane angle $(\theta)$, beam energy, and spectrometer angle. These acceptances are chosen to match the conditions of the real world Hall A experimental setup. Note that the initial SIMC solid angle variable acceptances $(\phi$ and $\theta$ ) were made $15 \%$ larger than the final cuts used in the analysis. This was done so that SIMC can apply a smearing function to these variables which more accurately reflects real world behavior. SIMC then randomly and uniformly generates particles in the detector in the provided acceptance ranges. Next SIMC calculates the energy of each electron produced, weights each event by a cross section model, and finally applies radiative corrections (see Section 3.8) to each event.

SIMC then transforms these events back through the spectrometer to their reaction vertex in the target. Along the way it applies energy losses due to multiple scattering as well as ionization and Bremsstrahlung. The spectrometer magnets are modelled using the COSY INFINITY program [24]. This program contains the various transformation matrices required to transport particles between the various coordinate systems and through the spectrometer. While being transported through the spectrometer SIMC checks to be sure that all recorded electrons do not strike the walls of the spectrometer or any non-sensitive elements of the detectors in the stack. It also checks that the 
electrons pass through all apertures in the spectrometer. SIMC also applies a smearing function to the electrons' VDC positions to match the real world VDCs [10].

Let us now compare the results of SIMC (red lines) to the real experimental data (blue lines). For all of these plots reasonable physics and acceptance cuts have been applied. The SIMC data was scaled arbitrarily to match the counts of the experimental data. We are only interested in comparing if SIMC matches the shape of the experimental data in the acceptance region of each variable. Figure 3.13 shows the SIMC result for $\phi$ (in-plane angle) alongside the experimental data. The agreement between SIMC and data seems to be fairly good, although the SIMC data falls off a little more quickly on the positive side. Figure 3.14 shows the SIMC result for $\theta$ (out-of-plane angle) alongside the experimental data. Once again the agreement looks decent, but there seems to be some drop off in the SIMC data in the negative $\theta$ direction. Figure 3.15 shows the SIMC result for $d P$ alongside the experimental data. Notice here that the SIMC data was scaled to match the experimental acceptance region of -0.02 to 0.03 . The agreement between SIMC and experiment outside of this region is poor, but in the region of our $d P$ acceptance cuts the shapes are in good agreement. The agreement of the shapes of the variables $\phi, \theta$, and $d P$ show that SIMC is reproducing experimental data well.

\subsection{Radiative Corrections}

In Chapter 1 the lowest order (Born term) Feynman diagram for elastic electron scattering was discussed. While this is a good first approximation, other diagrams contribute significantly to the cross section and must be accounted for as well. These diagrams can be categorized as external or internal radiative corrections. External corrections are characterized by interactions with other particles that are not the primary scattering source for the electron. These corrections come in the form of Bremsstrahlung radiation and ionization. Bremsstrahlung radiation is the radiation released as photons when electrons are slowed down by the Coulomb fields of nuclei in 


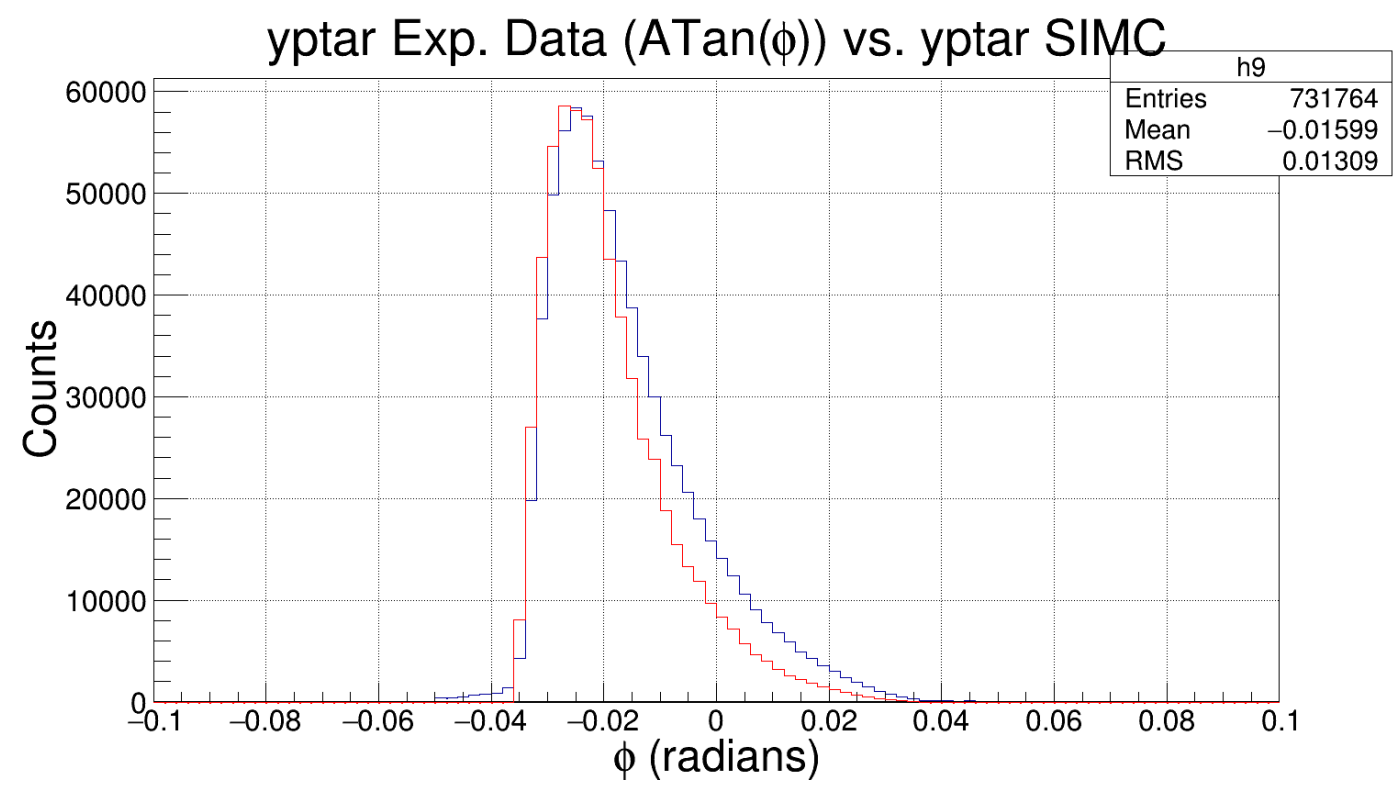

Figure 3.13: SIMC $\phi$ and Experimental Data $\phi . \phi$ is the in-plane angle. The red histogram is the SIMC data, and the blue histogram is the experimental data.

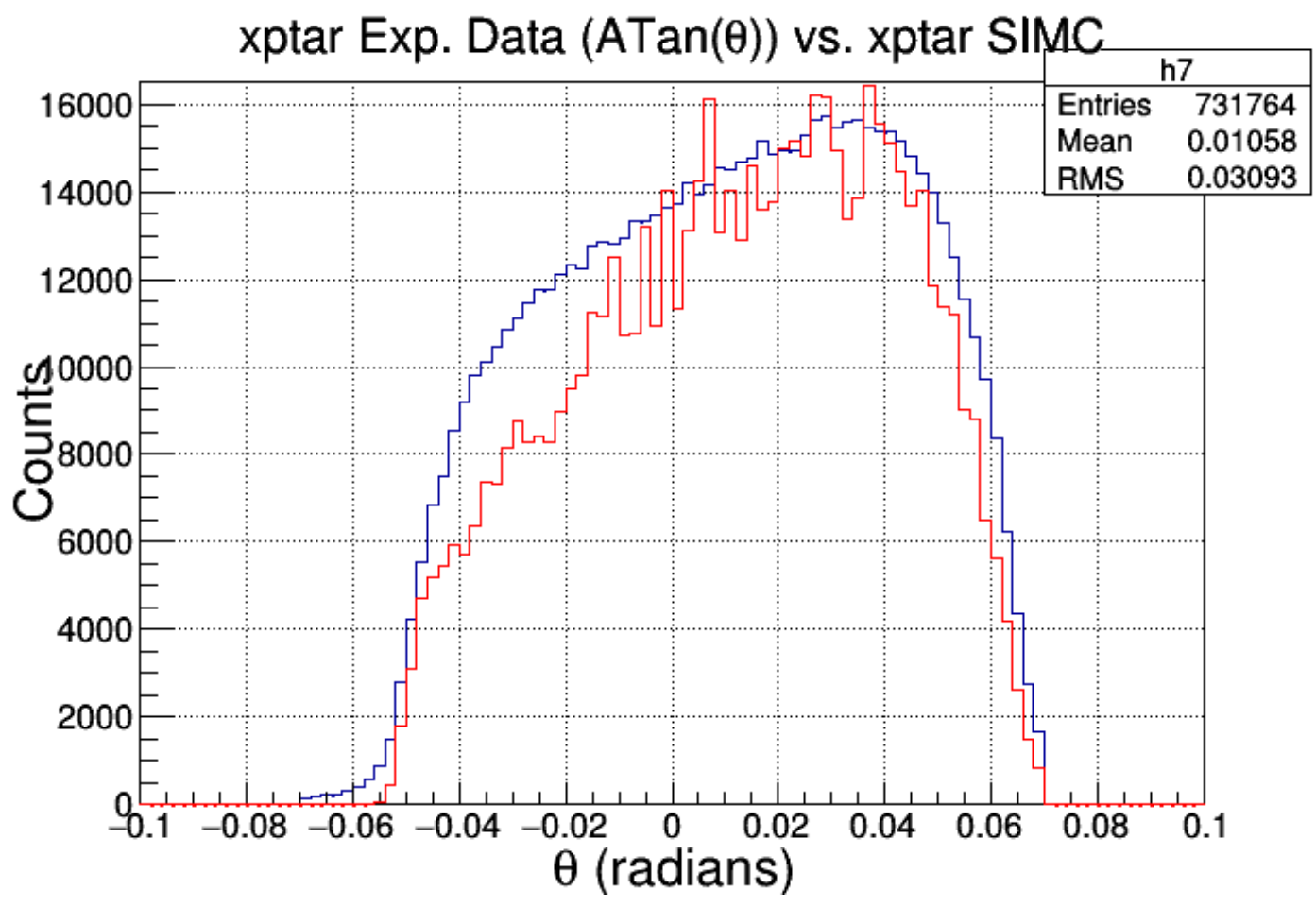

Figure 3.14: SIMC $\boldsymbol{\theta}$ and Experimental Data $\boldsymbol{\theta}$. $\theta$ is the out-of-plane angle. The red histogram is the SIMC data, and the blue histogram is the experimental data. 


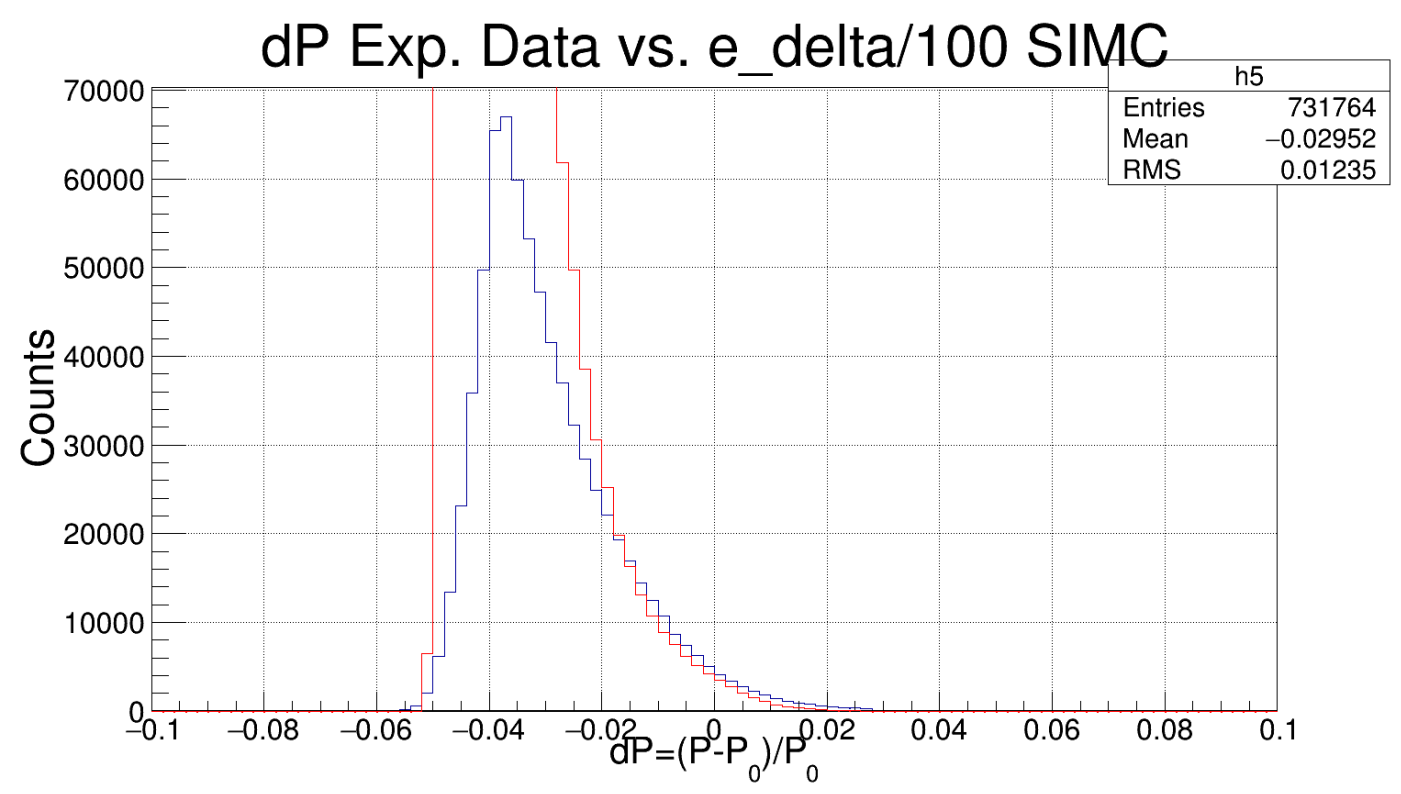

Figure 3.15: SIMC $\boldsymbol{d P}$ and Experimental Data $\boldsymbol{d P} . d P$ is the momentum fraction. The red histogram is the SIMC data, and the blue histogram is the experimental data.

the material the electron is passing through.

Internal corrections are characterized by the electron interacting with the primary scattering source via the exchange of real or virtual photons. Figure 3.16 shows the additional first order diagrams which must be accounted for along with the Born term diagram. These diagrams can again be separated into two categories, elastic and inelastic corrections. The elastic corrections exchange only virtual photons shown in Figure 3.16 b), c), and d). The inelastic corrections emit real photons as seen in e) [10].

The radiative corrections for E08-014 were calculated by the program XEMC. For a detailed discussion of the radiative corrections applied by XEMC see [25] and 26]. This program is derived from a previous program called RadCor which is detailed in 27] and 28. XEMC uses a built in cross section model to calculate the Born cross section, without radiative corrections, and the radiative cross section. XEMC also uses the peaking approximation discussed in 26.

These radiative corrections now allow us to compare our Monte Carlo elastic data 


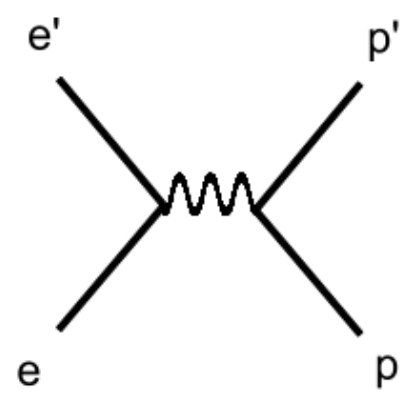

a) Born term

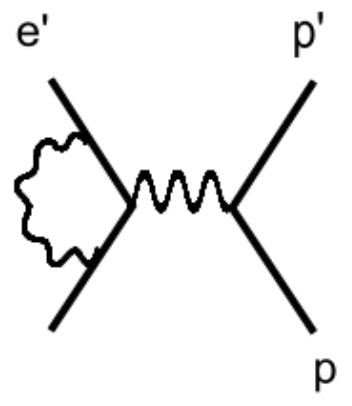

b) vertex

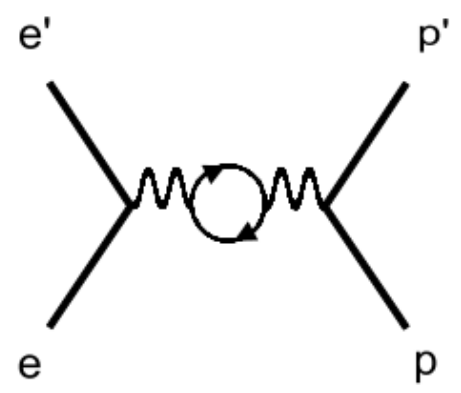

c) vacuum
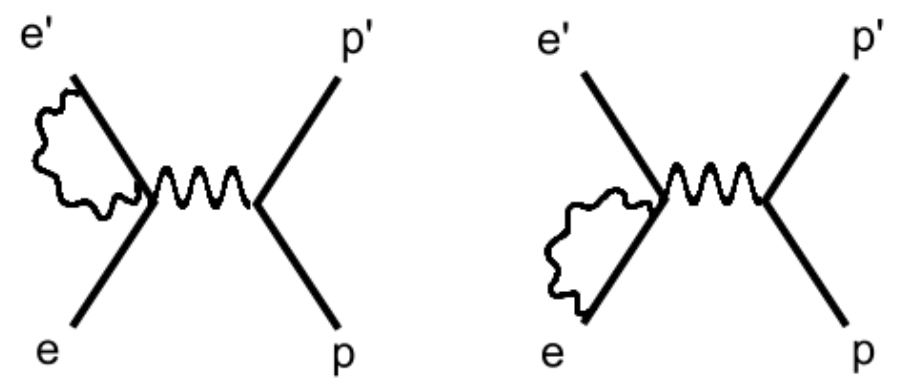

d) self energy
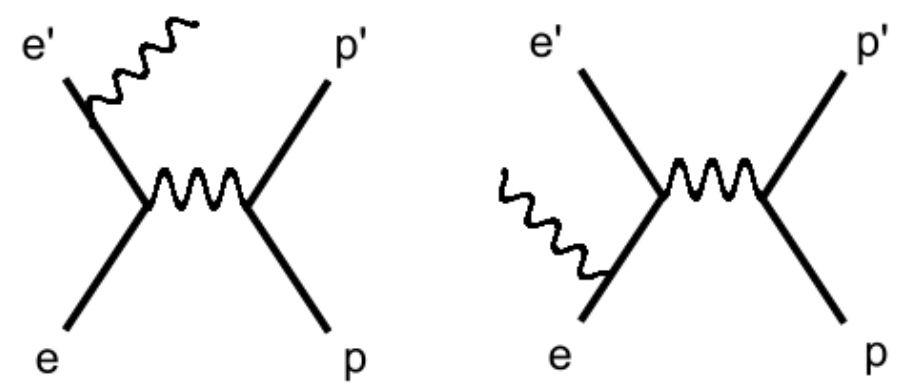

e) Bremsstrahlung

Figure 3.16: Born and Lowest Order Radiative Correction Diagrams. Image from 10 .

to our experimental data by means of Equation 3.13 . Where $\sigma_{R a d}^{E x p}$ is the experimental cross section, $\sigma_{\text {Born }}^{E x p}$ is the experimental Born cross section, $\sigma_{R a d}^{M o d e l}$ is the model radiative cross section, and $\sigma_{\text {Born }}^{M o d e l}$ is the model Born cross section 7 . Figure 3.19 shows the ${ }^{3} \mathrm{He}$ elastically scattered electron spectrum generated by SIMC in $x_{B j}$. The tail below the elastic peak is due to the radiative corrections, and demonstrates that the corrections 
are working as intended.

$$
\sigma_{\text {Born }}^{\text {Exp }}=\sigma_{\text {Rad }}^{\text {Exp }} \frac{\sigma_{\text {Born }}^{\text {Model }}}{\sigma_{\text {Rad }}^{\text {Model }}}
$$

\subsection{Aluminum Background Subtraction}

Many of the events detected by the experiment are derived from electrons scattering off of the aluminum walls of the target cell. These are not events we want in our cross section measurement so steps are taken to subtract this Al background out of the experimental data. This is done by means of a 'dummy' $\mathrm{Al}$ cell which is an empty $\mathrm{Al}$ target cell. This dummy cell is placed in the beam and the scattered electrons are measured as usual. Since the cell is empty all of the electrons measured scattered off of the $\mathrm{Al}$ in the cell showing what the $\mathrm{Al}$ background looks like without the ${ }^{3} \mathrm{He}$ target. After correcting for the differing radiative effects, Al thicknesses, and charge seen by the ${ }^{3} \mathrm{He}$ target and $\mathrm{Al}$ dummy cells the electrons scattered from $\mathrm{Al}$ can be subtracted from the production data. This yields an Al background subtracted result which contains only the electrons that scattered from ${ }^{3} \mathrm{He}$.

To subtract the $\mathrm{Al}$ from the dummy cell the total charge experienced by the dummy cell and the ${ }^{3} \mathrm{He}$ target cell must be the same. This means that the Al background must be scaled to account for the different amount of beam charge that the dummy data collected as compared to the production data. For this analysis the dummy cell result must be multiplied by 21.2708 to match the charge of the experimental runs. The dummy cell also had different Al wall thicknesses as compared to the target cell. To compensate for the larger dummy $\mathrm{Al}$ wall thickness the dummy data was scaled by another 0.1979 times.

When subtracting the $\mathrm{Al}$ background of the dummy cell from the ${ }^{3} \mathrm{He}$ cell one must take into account the different radiative corrections due to the differing $\mathrm{Al}$ thicknesses.

The Al dummy cell gives a radiative correction ratio of $\sigma_{\text {Born }}^{\text {Model }} / \sigma_{\text {Rad }}^{\text {Model }}=1.823$ and the ${ }^{3} \mathrm{He}$ target cell gives a ratio of $\sigma_{\text {Born }}^{\text {Model }} / \sigma_{\text {Rad }}^{\text {Model }}=1.467$. To account for the different 
radiative corrections the $\mathrm{Al}$ dummy background is scaled by the ratio of the dummy radiative corrections, 1.823 , to the target cell corrections, 1.467 . The resultant scaled Al background is shown in red in Figure 3.17. This red histogram is then subtracted from the blue histogram that represents the ${ }^{3} \mathrm{He}$ production data that is still contaminated by aluminum.

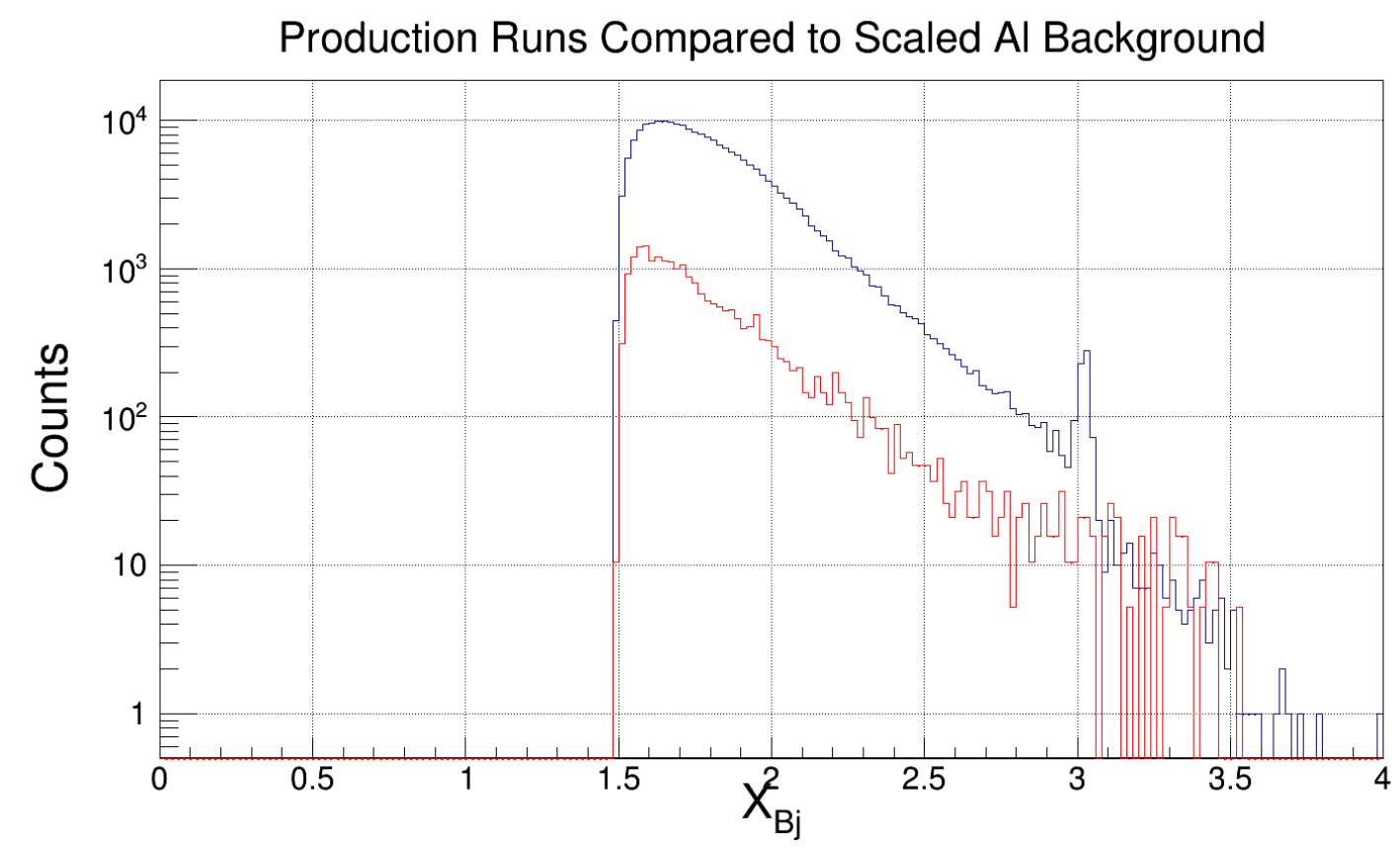

Figure 3.17: Scaled Aluminum Background. The red histogram shows the scaled $\mathrm{Al}$ background, and the blue histogram shows the ${ }^{3} \mathrm{He}$ production data before the $\mathrm{Al}$ background is subtracted.

Since the production data was taken on ${ }^{3} \mathrm{He}$ we do not anticipate seeing events at $x_{B j}>3$ since that is the elastic peak. However, there are clearly events in this region above the elastic peak. These events must be coming from something other than the ${ }^{3} \mathrm{He}$ gas. Notice that above the ${ }^{3} \mathrm{He}$ elastic peak the scaled Al background closely matches the production background. This is strong evidence that these events are coming from scattering off of the $\mathrm{Al}$ of the cell. Once the $\mathrm{Al}$ background is subtracted out most of the events above the ${ }^{3} \mathrm{He}$ elastic peak disappear leaving only the desired electrons that were scattered from the ${ }^{3} \mathrm{He}$ target. This can be seen in Figure 3.18 . 


\subsection{Electron Yields to Cross Sections}

At this point we have made numerous cuts and corrections to our data with the goal of getting a clean electron sample scattered from the ${ }^{3} \mathrm{He}$ target. Now we must use this data to obtain a cross section. We showed in Figure 2.4 in Section 2.2 that experiment E08-014 definitely captured elastically scattered electrons. We can determine how many electrons we detected after we apply all of the physics cuts and corrections to the $x_{B j}$ plot of the production runs.

To find the number of elastically scattered electrons we need to be able to count how many electrons are in the elastic peak. To accomplish this we need a method to fit the data such that the area of the elastic peak can be measured. The $x_{B j}$ plot can be broken down in to two areas, the quasielastic region and the elastic peak. We want to use one function to describe each region, and then combine these two functions to fit the relevant region of $x_{B j}$ with this combined fit.

Two functions immediately spring to mind as good candidates for the fit. Recall that the $x_{B j}$ plot is logarithmic, and notice that the quasielastic region sloping down to the elastic peak appears roughly linear on this log plot. This indicates that an exponential function will likely yield reasonable fit results for the quasielastic region. As we are only interested in elastic electrons we only need to fit the quasielastic region near the elastic peak $\left(x_{B j}>2.5\right)$. To fit the quasielastic region near the elastic peak a two parameter exponential function as in Equation 3.14 was chosen with $P_{0}$ and $P_{1}$ being free parameters.

$$
F_{\text {exp }}=e^{\left(P_{0}+P_{1} x\right)}
$$

Next we want to fit the elastic peak around $x_{B j}=3$. While a Poisson distribution may technically better describe the distribution of the electrons in the elastic peak a simple Gaussian describes the peak equally well. As such, Equation 3.15 was chosen to fit the elastic peak with free parameters $P_{0}, P_{1}$, and $P_{2}$. Finally by summing Equations 3.14 and 3.15 we have a combined fit that can be used to fit the region of $x_{B j}$ around 
the elastic peak. This combined fit is given in Equation 3.16 with free parameters $P_{0}$ through $P_{4}$.

$$
\begin{gathered}
F_{\text {Gaus }}=P_{0} e^{\left(-\frac{1}{2}\left(\frac{x-P_{1}}{P_{2}}\right)^{2}\right)} \\
F_{\text {combined }}=e^{\left(P_{0}+P_{1} x\right)}+P_{2} e^{\left(-\frac{1}{2}\left(\frac{x-P_{3}}{P_{4}}\right)^{2}\right)}
\end{gathered}
$$

Applying the combined fit to the $x_{B j}$ plot after applying physics cuts, corrections, and the aluminum background subtraction we get the result seen in Figure 3.18 , where the solid blue line is the combined fit. The combined fit seems to be doing a good job of capturing the quasielastic region before the elastic peak while also locating the elastic peak well. The fit is allowed to extend slightly beyond the peak for the purpose of creating some analysis histograms, but this extended region does not influence the number of electrons found in the elastic peak. We can also see that as we discussed in Section 3.9 once the Al background is subtracted out there are almost no events above the ${ }^{3} \mathrm{He}$ elastic peak.

The number of electrons under the Gaussian part of the combined fit is then the number of elastic electrons detected by the experiment prior to some corrections. After physics and acceptance cuts, but before live-time, GC efficiency, Trigger efficiency, and VDC efficiency corrections, experiment E08-014 detected 565 electrons elastically scattered from ${ }^{3} \mathrm{He}$. Table 3.7 shows a summary of the correction values applied to the number of electrons detected. Multiplying the uncorrected yield by all of these corrections factors we find the experimental yield of electrons elastically scattered from ${ }^{3} \mathrm{He}$ is 627 electrons.

Now we need to determine how this electron yield from the production data corresponds to a cross section value. To do this we will use the Monte Carlo simulation program SIMC discussed in 3.7. SIMC has a built in model of the ${ }^{3} \mathrm{He}$ cross section that has the correct shape of the form factors and cross section derived from older fits 


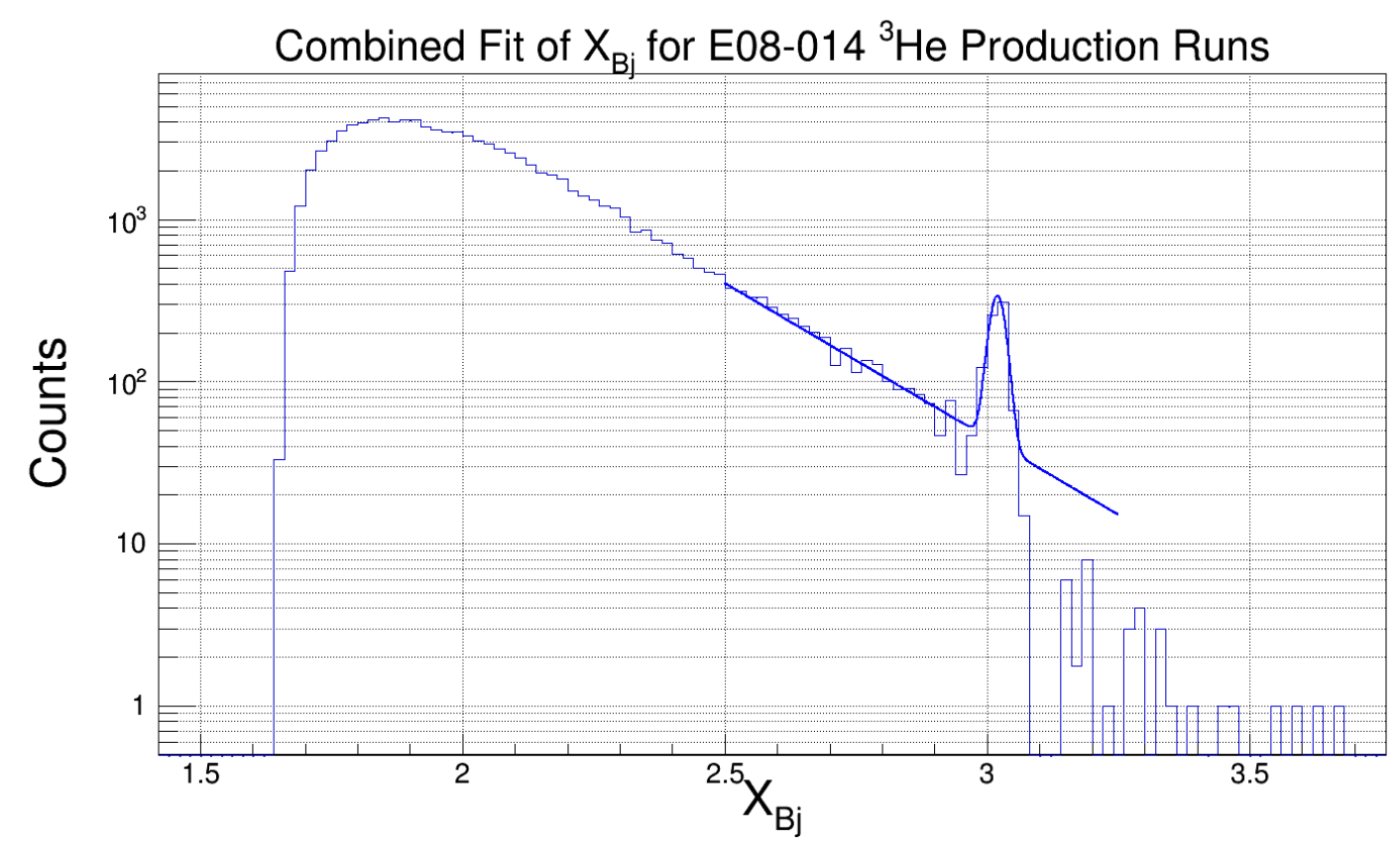

Figure 3.18: Combined Fit of $\boldsymbol{x}_{\boldsymbol{B} \boldsymbol{j}}$ for E08-014. The histogram is the plot of all of the E08-014 ${ }^{3} \mathrm{He}$ production runs containing elastic data after applying physics cuts, corrections, and the aluminum background subtraction. The solid blue line is the combined fit of an exponential, for the quasielastic region, and a Gaussian, for the elastic region.

\begin{tabular}{|r|ll|}
\hline Correction Type & Efficiency (\%) & Correction Factor \\
\hline Live-time & 95.27 & 1.050 \\
GC Efficiency & 96.55 & 1.036 \\
Trigger Efficiency & 98.858 & 1.0155 \\
VDC Efficiency & 99.175 & 1.0083 \\
\hline
\end{tabular}

Table 3.7: Summary of Correction Factors

of the ${ }^{3} \mathrm{He}$ world data [29]. We use SIMC to simulate electrons elastically scattered off of ${ }^{3} \mathrm{He}$ with the same energy, angle, charge, and acceptance cuts as our experiment. This purely elastic data is shown in Figure 3.19. The tail below the elastic peak at $x_{B j}=3$ is due to correcting for radiative effects. The tail falls of around $x_{B j}=1.6$ due to the acceptance limits of SIMC.

The goal at this point is to use the same combined fit we used for the production data to fit the SIMC data for a direct comparison. Clearly, having the elastic events from SIMC is not yet enough to match our experimental data since the SIMC data 


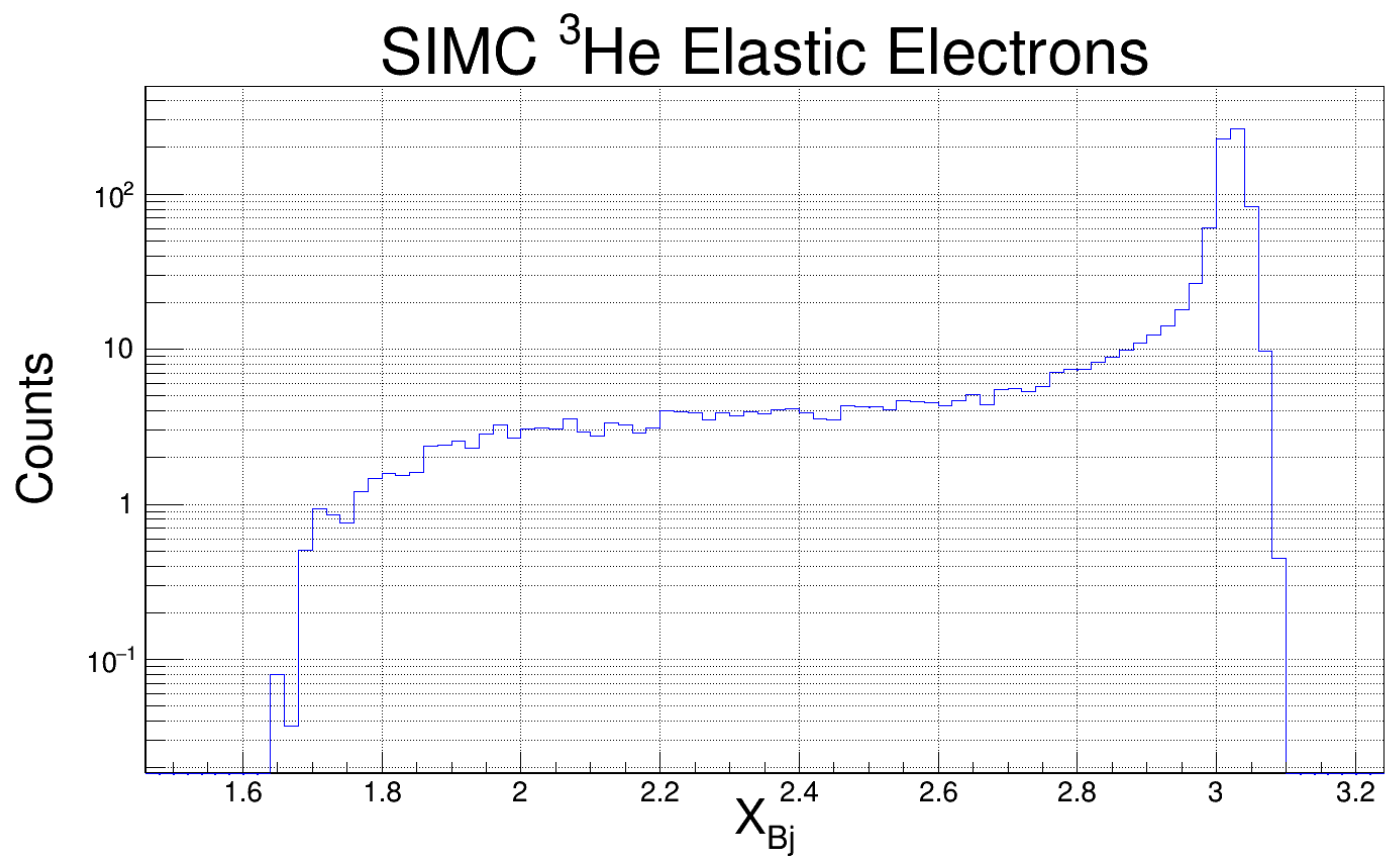

Figure 3.19: SIMC Elastically Scattered Electrons. These electrons were generated with the same kinematics, acceptances, and charge as experiment E08-014. The elastic peak is seen at $x_{B j}=3$ with a tail due to correcting for radiative effects below $x_{B j}=3$. The radiative tail falls off below $x_{B j}=1.6$ due to the acceptance limits of SIMC.

has no quasielastic events which make up the bulk of our dataset. To make the SIMC data comparable to the production data we need to add in the equivalent quasielastic events. This was done by taking the same type of exponential fit from Equation 3.14 and fitting the quasielastic region below the elastic peak of the production data. This exponential fit was done in the region where SIMC predicts there to be fewer than ten elastic electrons so as to only fit quasielastic data.

A histogram was then binned to this fit of the quasielastic data and can be seen in Figure 3.20 as the black histogram. Note that the fit is allowed to extend slightly beyond the elastic peak for the purpose of obtaining a good total fit, but this extended region does not influence the number of electrons found in the elastic peak. The new histogram representing only quasielastic data was then summed with the SIMC elastics only histogram in the region before and up to the ${ }^{3} \mathrm{He}$ elastic peak. This new combined 
SIMC and quasielastic histogram is then of the same shape as the production data in the region of interest allowing it to be fit with Equation 3.16. The areas under the Guassian portions of the combined fits are then directly proportional to the cross section values for SIMC and the production data since the elastic electron yields are proportional to the cross sections.

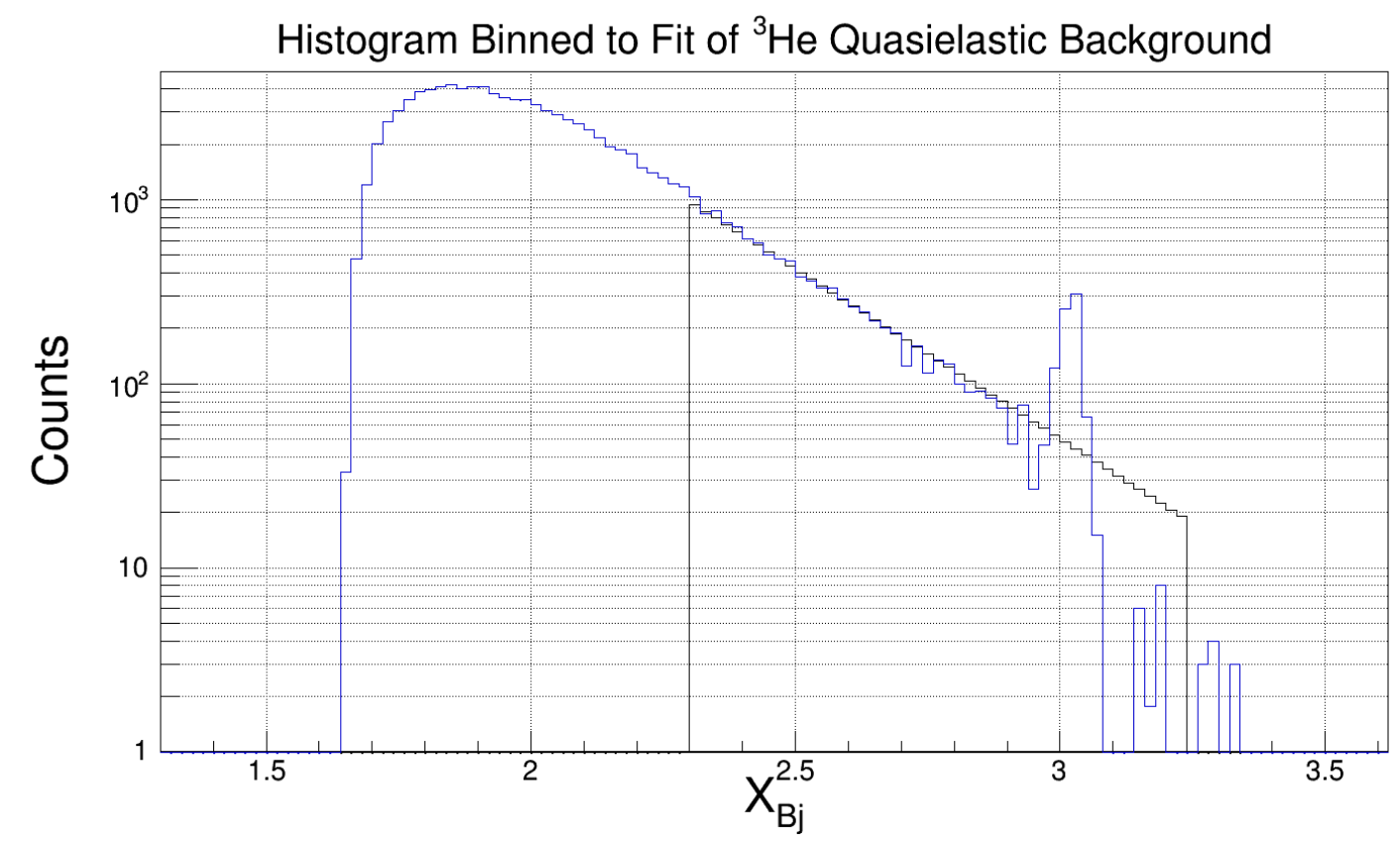

Figure 3.20: Histogram Binned to Fit of Quasielastic Background. The black histogram is binned to the fit of the quasielastic background of $x_{B j}$ without the elastic events. The blue histogram shows the production data including elastics with physics cuts for comparison.

While the shape of the form factors and cross section built into SIMC is correct the magnitude at $Q^{2} \approx 34.2 \mathrm{fm}^{-2}$ is likely off. This is why the SIMC elastic electron yield doesn't perfectly match the experimental data electron yield. So to find the cross section value of the production data we scale the SIMC elastic data by a constant magnitude up or down until the area of the Gaussian portion of the combined SIMC fit, the elastic electron yield, matches the area of the Gaussian portion of the combined fit of the production data. When the two Gaussian areas of the combined fits match the electron yields of SIMC and the experimental data then match meaning the cross 
sections are equivalent. This matching of the SIMC yield to the production yield leaves us with the scale factor, $C_{S I M C}$, we applied to the SIMC data to match the production data.

Since the Gaussian areas of the combined fits and the cross sections are directly proportional, and we have matched the Gaussian areas of SIMC and production data, we can multiply the cross section value in SIMC by $C_{S I M C}$ to find the cross section value of the production data. Figure 3.21 shows the $x_{B j}$ plot for the production data and its combined exponential and Gaussian fit in blue as well as the SIMC elastics histogram summed with the fitted quasielastic background histogram with its combined fit in red.

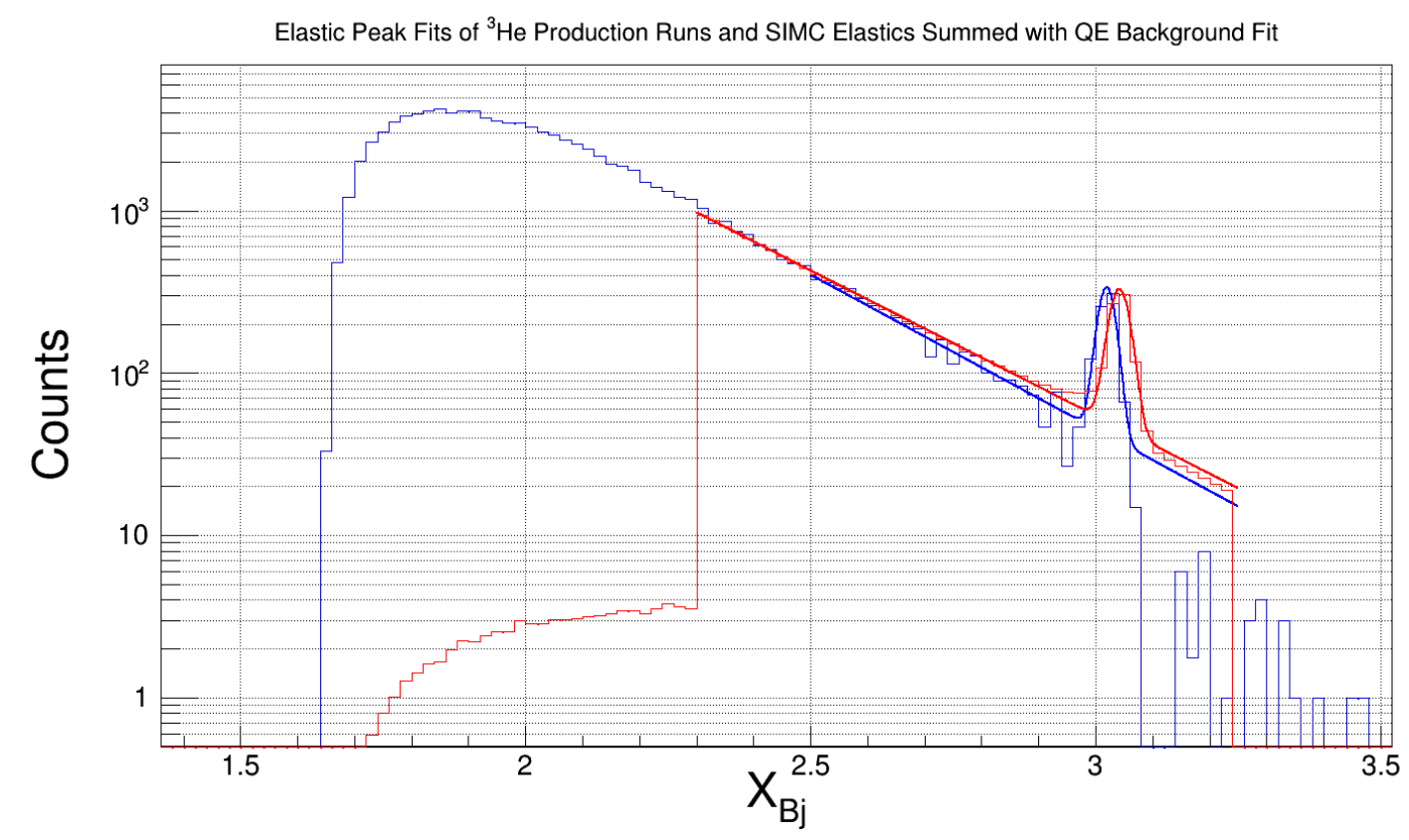

Figure 3.21: Elastic Peak Fits of ${ }^{3}$ He Production Runs and SIMC Elastics Summed with QE Background Fit. The blue histogram shows the production data for E08-014 with physics cuts, and its elastic peak is fit by the blue line. The red histogram is the sum of the SIMC elastics histogram from Figure 3.19 and the histogram binned to the fit of the quasielastic background from Figure 3.20, and it's elastic peak is fit by the red line.

Table 3.8 lists the parameters of the total fits to the production data and yield matched SIMC data. The SIMC elastic peak is slightly wider than the production 
peak indicating that the smearing function in SIMC may be tuned too high. The slight offset between the two elastic peaks is likely due to a small offset in $\mathrm{Z}_{\text {target }}$, however this should not influence the area under the elastic peak and thus not change the resultant cross section.

For this analysis the scale factor needed to match the yields, $C_{S I M C}$, was found to be 1.01984. This means that the model cross section needed to be increased by $1.984 \%$ to match the experimental data. When this adjustment is made the model built into SIMC will then yield the cross section for elastic scattering off of our ${ }^{3} \mathrm{He}$ production data. This cross section is found to be $1.335 \times 10^{-10} \mathrm{fm}^{2} / \mathrm{sr}$ or $1.335 \times 10^{-6} \mu \mathrm{b} / \mathrm{sr}$.

\begin{tabular}{|c|ccccc|}
\hline Combined Fit Par: & $\mathbf{P}_{0}$ & $\mathbf{P}_{1}$ & $\mathbf{P}_{2}$ & $\mathbf{P}_{3}$ & $\mathbf{P}_{4}$ \\
\hline Production Data: & 17.0 & -4.38 & 297 & 3.02 & -0.0152 \\
SIMC Elastics Plus & 16.4 & -4.12 & 282 & 3.04 & -0.0177 \\
QE Background Fit & & & & & \\
\hline
\end{tabular}

Table 3.8: Combined Exponential and Gaussian Fit Parameters

To test if the scale factor $\left(C_{S I M C}\right)$ is reasonable we can remake the SIMC elastic histogram with the SIMC cross section model multiplied by $C_{S I M C}$. Once a new set of elastic events are generated we can rerun the yield matching code with the new Monte Carlo results. If our scale factor applied to the cross section is correct we would expect the yields of the production data and the new SIMC elastics summed with the QE background to exactly match without any scaling needed (i.e. $C_{S I M C}=1$ ). This test was performed and the electron yields were found to match with $C_{S I M C}=1$ as desired.

\subsection{Bin Centering Corrections}

Now that we have found the magnitude of our ${ }^{3} \mathrm{He}$ elastic cross section we must ask, "Where should we place this data point in $Q^{2}$ ?" The immediately intuitive answer to this question is that we should place this point at the center of our bin in $Q^{2}$. However, this is not the correct place for our data point because the shape of the cross section 
across the range of our $Q^{2}$ bin is not linear.

To find the correct location for our data point in $Q^{2}$ we will follow the procedure laid out in [30]. To see the issue with placing our point at the center of our bin let us look at a plot of our cross section at $3.356 \mathrm{GeV}$ seen in Figure 3.22. Note that the specific form factors chosen for this cross section are chosen as a representative fit for the group of 'good' sum of Gaussian fits as discussed later in Section 4.3.3. A 'good' fit here was determined by having a low $\chi^{2}$ value and more importantly the physical characteristics we would expect from a form factor. For a plot of the new cross section fit, the previous cross section fit from [29], and the ${ }^{3} \mathrm{He}$ cross section measured in this analysis see Section 4.3 .3 and Figure 4.13

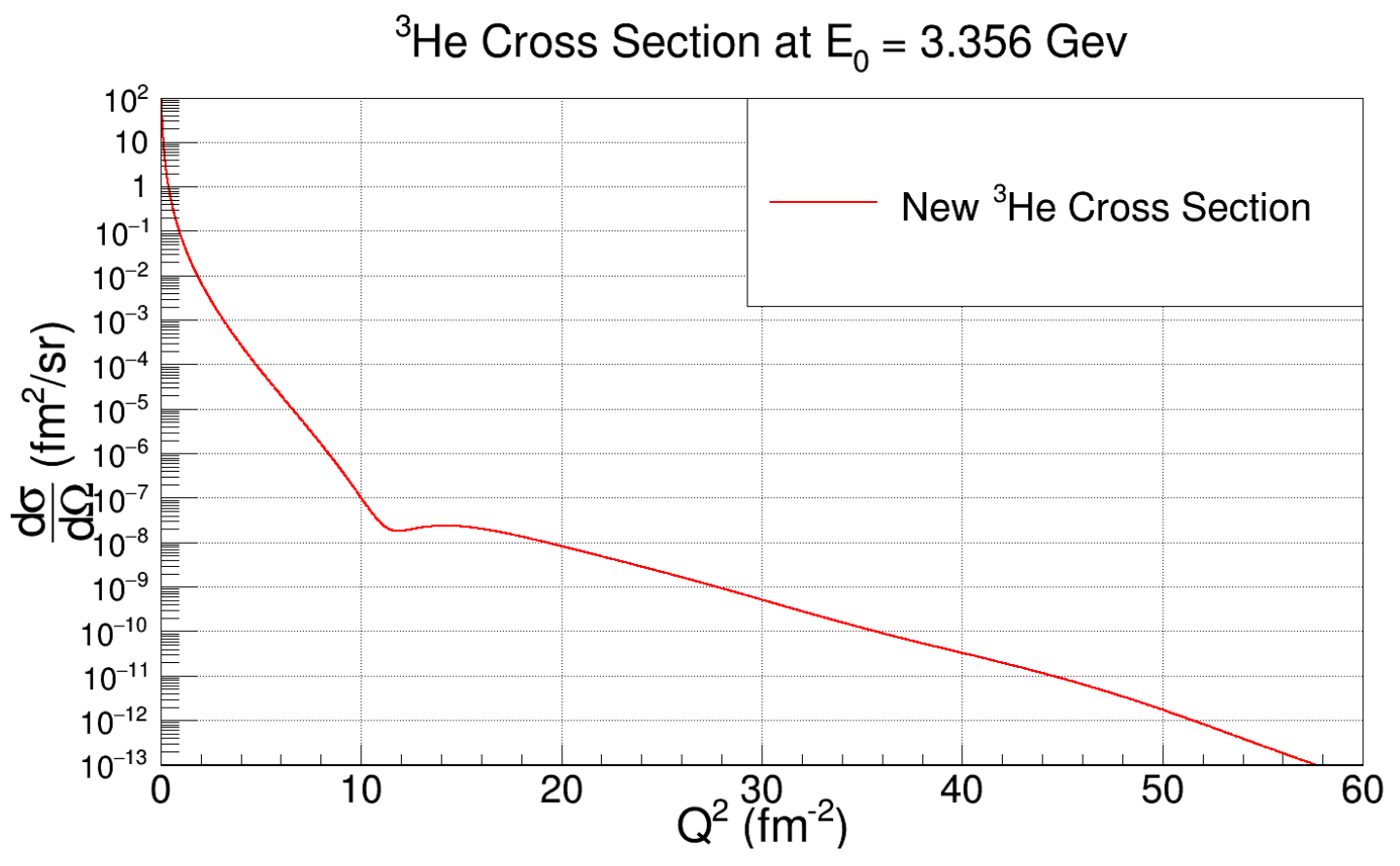

Figure 3.22: ${ }^{3} \mathbf{H e}$ Elastic Cross Section at $\boldsymbol{E}_{\mathbf{0}}=\mathbf{3 . 3 5 6} \mathrm{GeV}$ This cross section was produced using a representative fit from the sum of Gaussians fits discussed in Section 4.3 .3 . Notice that the shape of the log plot looks linear in the region of $Q^{2} \approx$ $35 \mathrm{fm}^{-2}$.

We know our point should be located somewhere in the neighborhood of $Q^{2} \approx 35$ $\mathrm{fm}^{-2}$ simply from the kinematics of the experiment. Looking around $Q^{2} \approx 35 \mathrm{fm}^{-2}$ in Figure 3.22 the plot appears linear. However, the plot is in a log scale indicating that 
the actual shape is an exponential. To see the true shape of the cross section let us first define our bin in $Q^{2}$, and then zoom in on our region of interest while removing the log on the $Y$-axis.

To find our bin size in $Q^{2}$ we can use our cuts on the in-plane angle $\phi$. Since we know the beam energy we can calculate $Q^{2}$ using Equations 1.3 and 1.1 knowing that $\phi$ is the analyzer variable for the arctangent of the deviation from the set spectrometer scattering angle. Table 3.9 shows the size of our analysis bin in various units. Figure 3.23 shows the cross section in the region of the analysis bin with the log plot removed to show the true shape.

\begin{tabular}{|c|cc|}
\hline Variable & Bin Minimum & Bin Maximum \\
\hline $\begin{array}{c}\text { Analyzer } \phi \text { (Radians) } \\
\text { Deviation from }\end{array}$ & -0.03 & 0.03 \\
Spectrometer Angle & -0.29991 & 0.29991 \\
(Radians) & & \\
Angle (Radians) & 0.3372 & 0.3972 \\
Angle (Degrees) & 19.32 & 22.75 \\
$Q^{2}\left(\mathrm{GeV}^{2}\right)$ & 1.188 & 1.604 \\
$Q^{2}\left(\mathrm{fm}^{-2}\right)$ & 30.55 & 41.24 \\
\hline
\end{tabular}

Table 3.9: Bin Width at $3.356 \mathrm{GeV}$ and Spectrometer Setting of $21.04^{\circ}$

Figure 3.23 makes it clear that the cross section across our bin is not linear, and thus taking the average $Q^{2}$ of the bin to set our cross section's $Q^{2}$ value is incorrect. Instead we must account for the fact that the shape of the cross section biases our data towards lower $Q^{2}$. This can be done by taking a weighted average of the $Q^{2}$ values in our bin where the weights are the cross section values at each $Q^{2}$. Performing this calculation we find that the weighted average $Q^{2}$ for our analysis bin is $34.19 \mathrm{fm}^{-2}$. This is a significant deviation from the bin center of $35.90 \mathrm{fm}^{-2}$. We now see that as expected the shape of the cross section requires us to place our data point at a lower $Q^{2}$ than the bin center. 


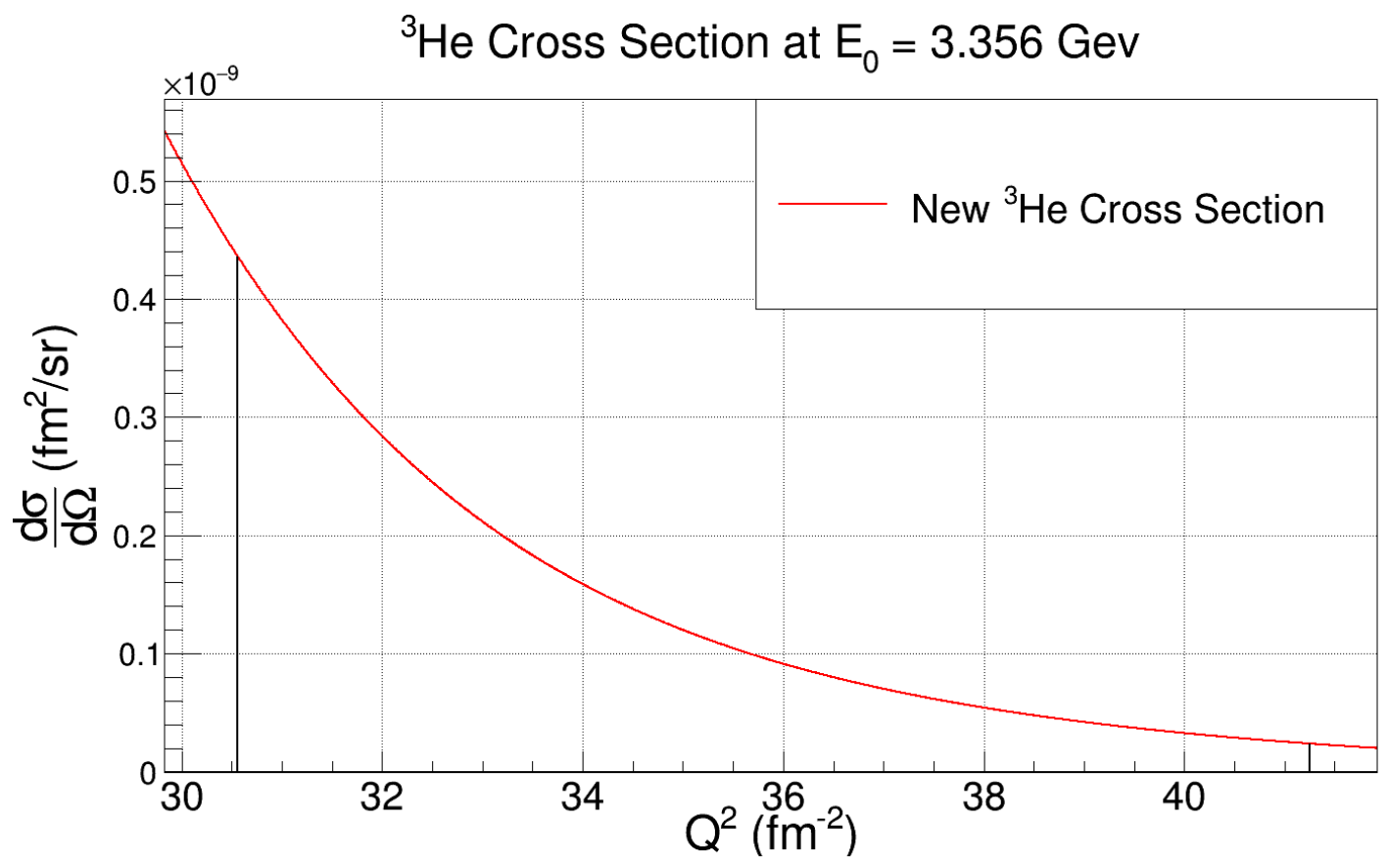

Figure 3.23: ${ }^{3}$ He Elastic Cross Section $Q^{\mathbf{2}}$ Bin at $3.356 \mathbf{G e V}$ This plot shows the same cross section as Figure 3.22 , except zoomed in on the analysis bin region with the $\log$ on the $Y$-axis removed. The two vertical black lines represent the minimum and maximum $Q^{2}$ of the analysis bin.

\subsection{Uncertainty}

Thus far, we have found the magnitude of the ${ }^{3} \mathrm{He}$ elastic cross section and determined where to place this data point in $Q^{2}$. Our next duty is to quantify the various sources of uncertainty that apply to our cross section. The uncertainty can be broken in to statistical uncertainty and systematic uncertainty. Statistical uncertainty describes the innate statistical fluctuations of measurements of an observable due to the inherent limitations of the tool making the measurements and the number of measurements made. Statistical uncertainty can be decreased by increasing the number of measurements made.

Systematic uncertainty describes uncertainty introduced from measuring instruments or incorrectly calibrated simulation tools or models. Systematic uncertainty can also come from unpredictable experimental conditions like environmental noise. Systematic errors are reproducible and generally in the same direction so taking more 
measurements will not significantly reduce them. This section will go into detail on how each source of uncertainty was quantified. Table 3.10 contains a summary of the experimental uncertainties on the ${ }^{3} \mathrm{He}$ elastic cross section that are described in detail below.

\begin{tabular}{|l|r|}
\hline Uncertainty Source & $\begin{array}{r}\text { Cross Section } \\
\text { Uncertainty }\end{array}$ \\
\hline Statistical Sources & $4.21 \%$ \\
\hline Electron Yield & $1.1 \%$ \\
Al Background Subtraction & $\mathbf{4 . 3 6} \%$ \\
Total Statistical & \\
\hline Systematic Sources & $3.08 \%$ \\
\hline Target Density & $2.25 \%$ \\
Optics Calibration & $1.32 \%$ \\
GC Efficiency & $1.1 \%$ \\
Beam/Target Offsets & $1 \%$ \\
Radiative Corrections & $1 \%$ \\
Beam Charge & $1 \%$ \\
VDC Single-Track Efficiency & $1 \%$ \\
Trigger Efficiency & $0.72 \%$ \\
Beam Energy & $0.5 \%$ \\
SIMC Model Comparison to Reality & $0.055 \%$ \\
PR Cut & $0.045 \%$ \\
Ytarget Position & $0.0115 \%$ \\
Live-time & $\mathbf{4 . 7 2} \%$ \\
Total Systematic & $\mathbf{6 . 4 2} \%$ \\
\hline \multicolumn{2}{|c|}{ Total Uncertainty } \\
Statistical \& Systematic
\end{tabular}

Table 3.10: Table of Uncertainties

First let us examine our statistical uncertainties. Our primary source of statistical uncertainty is derived from our yield of elastic electrons. As discussed in Section 3.10 experiment E08-014 had a yield of 565 electrons elastically scattered off of ${ }^{3} \mathrm{He}$. The uncertainty on this value is described by Equation 3.17, where $N_{e}$ is the number of elastically scattered electrons. The uncertainty in the number of electrons is directly proportional to the cross section. Thus, the statistical uncertainty on the cross section due to the electron yield is $4.21 \%$. 


$$
\delta_{e}=\frac{1}{\sqrt{N_{e}}}
$$

The other source of statistical error comes from the aluminum background subtraction discussed in Section 3.9. By subtracting out the Al contamination in the region of the elastic peak we have necessarily introduced another opportunity for uncertainty to creep into our measurement. In the region of the elastic peak, defined as $2.95<x_{B j}<3.10$, there were 1093 events prior to the Al background subtraction. In this same region there were $34 \mathrm{Al}$ events. We can once again use Equation 3.17 to estimate the uncertainty on the $\mathrm{Al}$ events. We find that these 34 events have an uncertainty of $17 \%$ or \pm 6 electrons.

This is a high uncertainty on a small number so to have a conservative estimate the uncertainty was doubled from \pm 6 electrons to \pm 12 electrons. When the $\mathrm{Al}$ background is subtracted from the ${ }^{3} \mathrm{He}$ production data we have either 1071 (1093 - 34 + 12) electrons remaining or 1047 (1093 - 34 - 12) electrons remaining. The uncertainty is then \pm 12 electrons from the mean number of electrons remaining after the $\mathrm{Al}$ subtraction. This gives $1059 \pm 12$ electrons yielding an uncertainty of $1.1 \%$. Note that this uncertainty is categorized as statistical in nature. It could be reasonably argued to be systematic, but as taking more production data would lower this uncertainty it has been categorized as statistical.

Next we will account for the systematic uncertainties on our cross section result. The largest source of systematic uncertainty comes from the density of the gaseous ${ }^{3} \mathrm{He}$ in the target cell. This issue was discussed in Section 3.5. The density study performed in 23] lists the uncertainty on the ${ }^{3} \mathrm{He}$ density as $3.08 \%$ which will apply directly to the cross section as the cell density is directly proportional to the cross section.

The next largest source of systematic uncertainty comes from the optics calibration of the HRSs. This calibration was discussed in Section 2.10.2. The precision of this calibration is limited by the physical characteristics of the optics sieve plate used for 
the calibration shown in Figure 2.31. The optics calibration works by locating where electron events fall within the holes of the sieve to create a pattern which can be found in the detector, and then be connected back to the target coordinate system.

These electron events fall randomly anywhere inside a given sieve hole. By using the outermost holes of the sieve with data inside of them one can define an area inside which all the optics data is found. The precision of the measurement of the size of this internal area is limited by the size and spacing of the sieve holes. This gives an uncertainty on the area containing data within the sieve. This area corresponds to the uncertainty in the solid angle from the optics calibration. The solid angle includes the uncertainties of both the in-plane angle $(\phi)$ and out-of-plane angle $(\theta)$ as well as the momentum $d p$ since that is directly related to $\phi$ for elastic scattering.

To estimate the inherent uncertainty of the optics calibration a Monte Carlo simulation was built. This simulation creates a sieve of $N$ by $M$ holes. Events are then randomly placed in each hole. The electron events placed in holes on the outer edge of the sieve are then connected by a straight line to their adjacent outer edge hole's electron event. Once all of the outer edge holes are connected they create a polygon whose internal area can then be calculated. This process is shown for a $5 \mathrm{X} 5$ sieve in Figure 3.24, where there are five of these randomly determined areas shown.

This process is repeated one million times resulting in one million different possible areas based on the hole sizes, spacings, and where the events were randomly placed. A histogram of all of these possible areas can then be created. This histogram forms an excellent Guassian shape which can then be fit with a Gaussian function. From this Guassian function a standard deviation (one $\sigma$ ) can be found to estimate the uncertainty of the area. The plot of one million randomly generated areas using the geometry of Figure 3.24 is shown in Figure 3.25. By fitting a Gaussian to the histogram of areas the standard deviation is found to be $30.2 \mathrm{~mm}^{2}$ on an area of $5000 \mathrm{~mm}^{2}$. For this analysis a two $\sigma(95 \%)$ confidence interval was chosen giving an uncertainty of $60.4 \mathrm{~mm}^{2}$ on $5000 \mathrm{~mm}^{2}$ or $1.21 \%$ in this example.

This is almost sufficient to estimate the optics calibration uncertainty. However, 


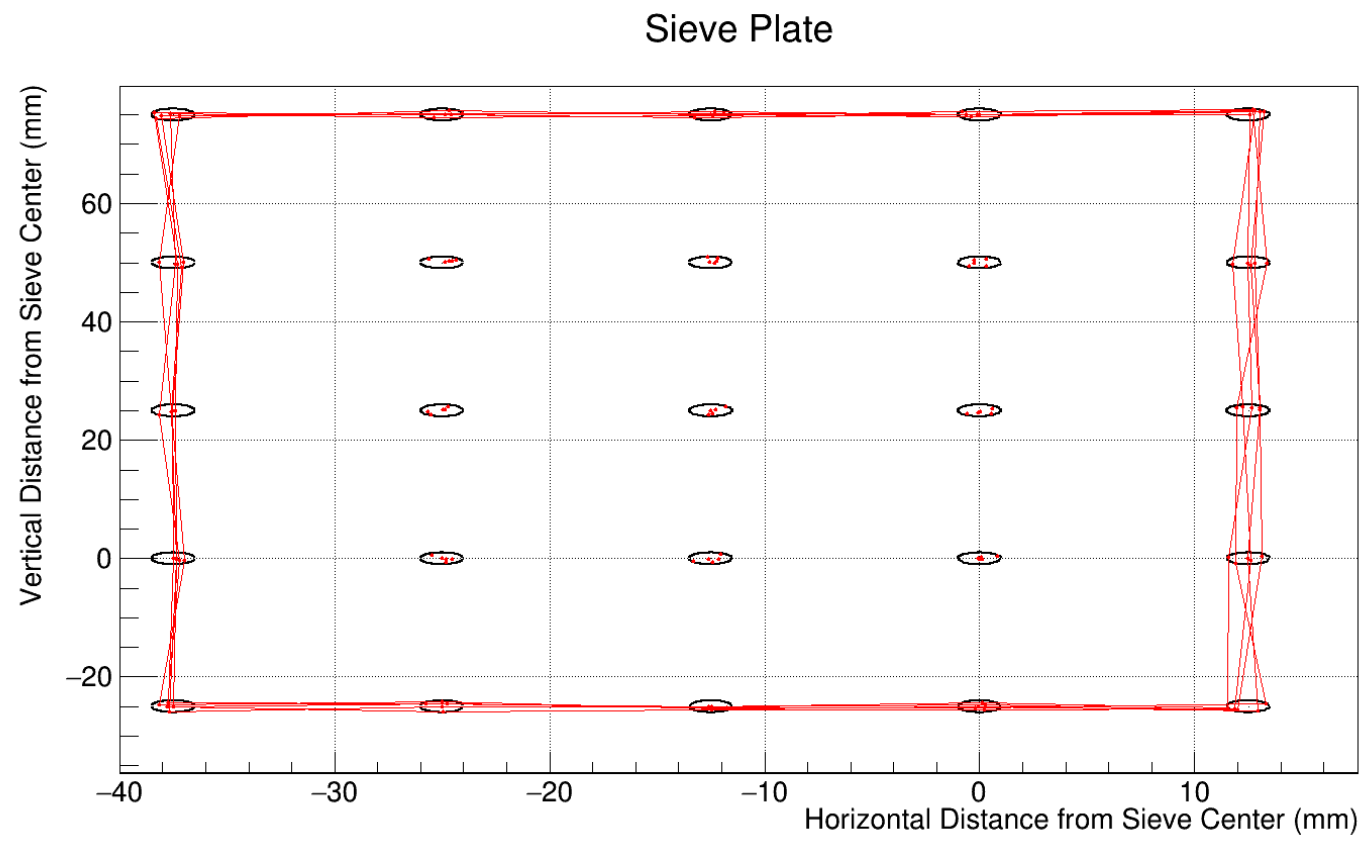

Figure 3.24: Optics Sieve Monte Carlo This figure represents a 5 X5 hole region of the optics sieve. The red dots are randomly placed events and the five connecting red lines enclose the outer area of the sieve defining the region of the optics data.

we can do better than estimating the whole acceptance using one sieve size. This is because as one steps through the target cell in $Y_{\text {target }}$ the solid angle acceptance, and thus the sieve size, changes as shown in Figure 3.26. So to accurately estimate the sieve uncertainty an optics run with similar kinematic settings to this analysis' kinematics was studied [31].

This optics run contained 11 carbon foils evenly space in $Y_{\text {target }}$. By finding how many holes each foil had significant data in the Monte Carlo could be configured to match the solid angle acceptance for each of the 11 slices of $Y_{\text {target }}$. The Monte Carlo was then run one million times for each of these solid angles, and the results of the two $\sigma$ area uncertainties were added in quadrature to find the total solid angle uncertainty. This overall two $\sigma$ uncertainty was found to contribute $2.25 \%$ uncertainty to the final cross section.

The next largest systematic uncertainty comes from the GC efficiency given by Equation 3.7 discussed in Section 3.4.5. The uncertainty on this efficiency is directly 


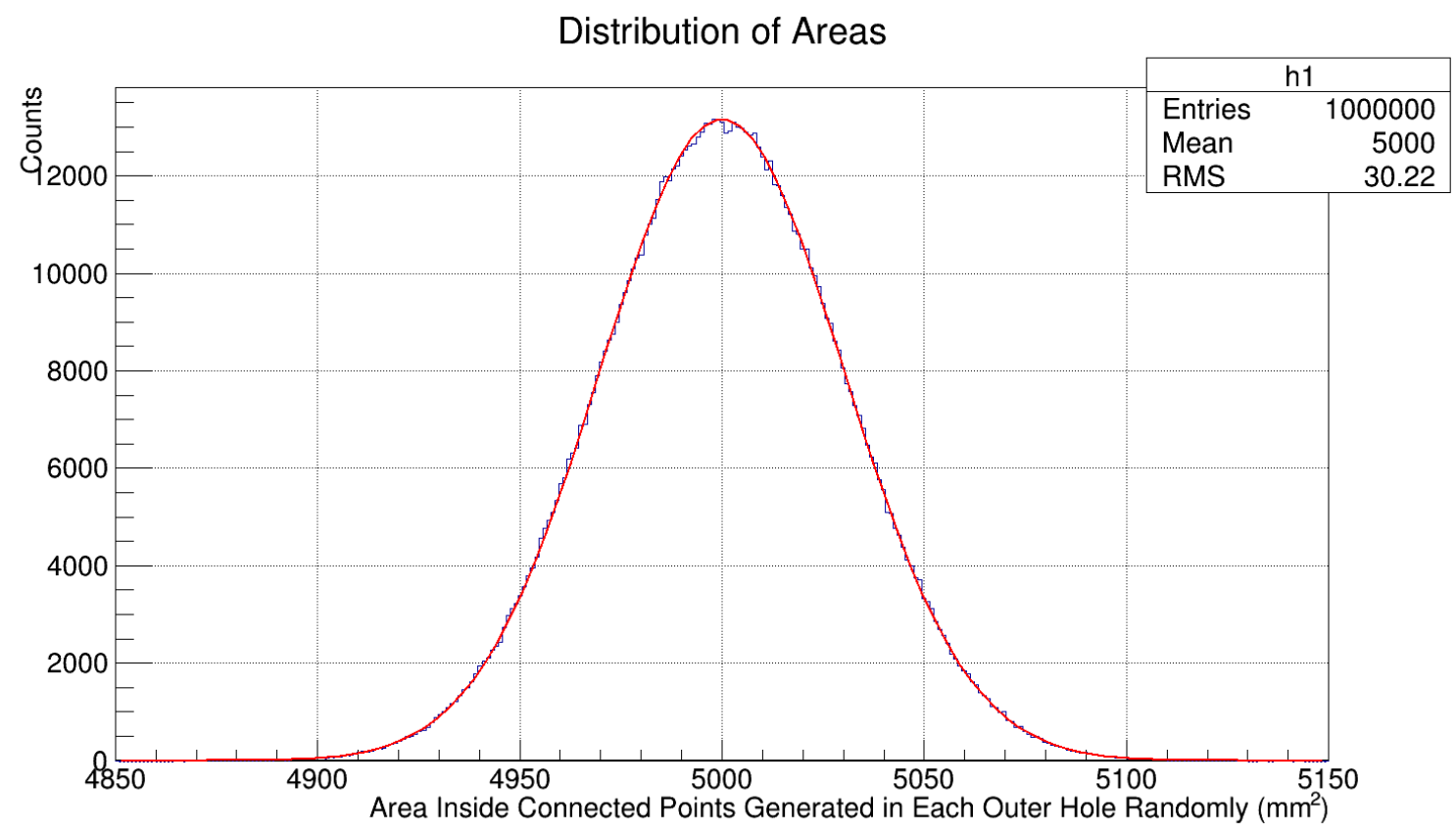

Figure 3.25: Optics Sieve Monte Carlo Areas Histogram This figure shows the results of one million areas created by placing points randomly in the outer sieve holes and connecting them to their nearest neighbor. It is fitted with a Guassian in red to find the standard deviation.

proportional to the uncertainty in the cross section and is given in Equation 3.18 from 21. Here $\epsilon_{G C}$ is the efficiency of the $\mathrm{GC}, \mathrm{T}_{3}$ is the number of events measured by the main trigger, $\mathrm{T}_{7}$ is the number of events measured by the coincidence trigger, and $\mathrm{T}_{3 \& 7}$ is the number of times both triggers fired. Inserting the relevant values yields an uncertainty on the cross section of $1.32 \%$.

$$
\delta_{G C}=\epsilon_{G C} \frac{\sqrt{T_{7}-\left(T_{3 \& 7}\right)}}{T_{7}}
$$

There is also a systematic uncertainty introduced by the beam and target offsets with respect to the set spectrometer angle. To estimate this uncertainty the Mott cross section was calculated at E08-014's energy and two angles. One angle was 1 mrad above the set spectrometer angle, and the other value was set $1 \mathrm{mrad}$ below the set spectrometer angle. The difference in these two Mott cross sections then closely represent the uncertainty a reasonably sized offset would introduce to the absolute 

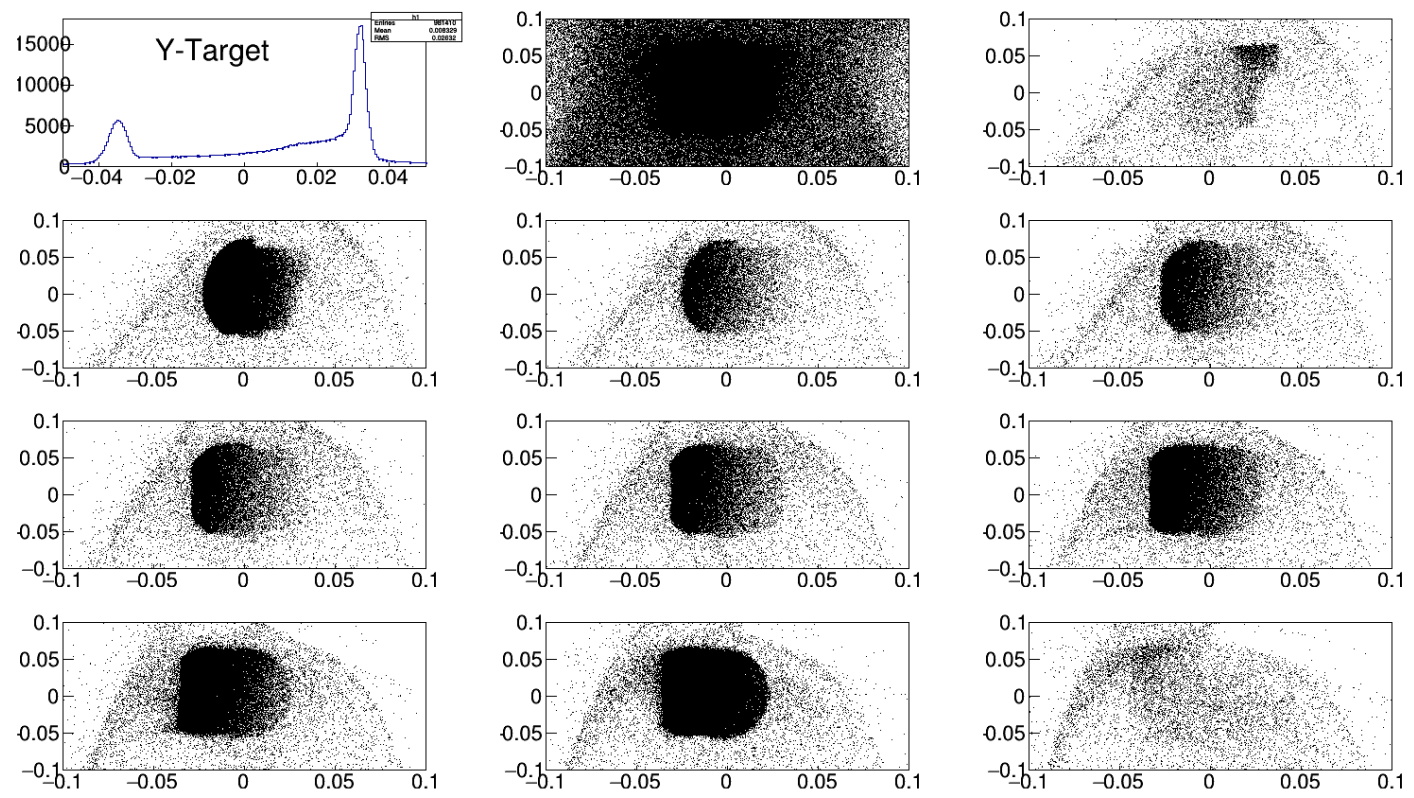

Figure 3.26: Solid Angle Acceptance of ${ }^{3} \mathbf{H e}$ Cell in $\boldsymbol{Y}_{\text {target }}$. The top left plot shows the electron events distributed in $Y_{\text {target }}$. The second from the top left shows the solid angle acceptance ( $\theta$ vs. $\phi)$ for all of $Y_{\text {target }}$. The remaining plots show the solid angle acceptance at different slices of $Y_{\text {target }}$ every centimeter in $Y_{\text {target }}$ from $Y_{\text {target }}=-5 \mathrm{~cm}$ to $Y_{\text {target }}=5 \mathrm{~cm}$. The important thing to notice is that the solid angle acceptance is not constant along $Y_{\text {target }}$.

cross section. This beam and target offset uncertainty is found to be $1.1 \%$.

The systematic uncertainty on the cross section due to radiative corrections in SIMC has been shown to be on the order of $1 \%$ [10]. The uncertainty on the cross section due to the beam charge measurement from the BCMs has also been shown to be $1 \%$. Both the VDC single track efficiency uncertainty and the trigger efficiency uncertainty are directly proportional to the cross section, and have both been estimated to be $1 \%[7]$.

The next largest systematic uncertainty comes from the uncertainty in the beam energy. The beam energy measurement is currently known to have an uncertainty of $0.04 \%$ [32]. However, this is not the uncertainty contributed to the cross section from the beam energy. To find the uncertainty on the cross section SIMC was run two more times with the same cuts and experimental conditions as previously except the beam energy was set $0.04 \%$ higher once and $0.04 \%$ lower once. The resulting cross 
sections from SIMC then given an upper and lower bound on the cross section due to fluctuations in beam energy. The uncertainty on the cross section from the uncertainty in the beam energy was then found to be $0.72 \%$.

The cross section model in SIMC also introduces some uncertainty to our cross section. This uncertainty has been previously studied in detail, and was found to contribute approximately $0.5 \%$ uncertainty to the cross section 33 . Note that binning the cross section in $x_{B j}$ gives less of a change in cross section over the bin than if the data had been binned in $E^{\prime}$. This helps to reduce the uncertainty on the cross section from the SIMC model.

The remaining systematic uncertainties are fairly small. One of these is the degree of uncertainty introduced by the pion rejector cut applied to the data. The 'good electron' region of the GC spectrum where the events are almost certainly electrons is defined as ADC channels 300-500. In this region there were 51439 events. Applying the PR cut removes 777 of these events from the sample. Using equation 3.17 the uncertainty on these 777 events is found to be \pm 28 events. The maximum events remaining after the PR cut is then $50690(51439-777+28)$, and the minimum number is 50634 (51439 - $777-28)$. The average number of events remaining after the PR cut is then $50662 \pm 28$ which gives an uncertainty to the final cross section of only $0.055 \%$.

Some uncertainty is introduced from the $Y_{\text {target }}$ positioning. One can never do better than finding the location of a single optics foil when calibrating $Y_{\text {target }}$. These foils can be found with approximately $0.2 \mathrm{~mm}$ accuracy. For our $20 \mathrm{~cm}$ target this gives an uncertainty of $0.1 \%$ on the $Y_{\text {target }}$ position. As we did with the beam energy uncertainty we ran SIMC twice more with the minimum and maximum possible $Y_{\text {target }}$ offsets and compared the uncertainty this introduces to the cross section. The uncertainties are asymmetric in this case with the positive $Y_{\text {target }}$ uncertainty being $0.045 \%$ and the negative being $0.017 \%$. Both values are quite small and barely influence the cross section at all. We will conservatively say that this can introduce up to $0.045 \%$ uncertainty to the final cross section.

The final source of measurable uncertainty comes from the live-time calculation 
discussed in Section 3.4.1. The uncertainty on the live-time can be calculated as in Equation 3.19, where $\epsilon_{L T}$ is the live-time for the experiment, $T_{3_{\text {Scaler }}}$ is the scaler number of main triggers $\left(T_{3}\right)$, and $T_{3_{F i l e}}$ is the electronics output number of main triggers detected. This value applies directly to the cross section. Inserting the relevant values the uncertainty on the cross section due to the live-time uncertainty is found to be $0.0115 \%$.

$$
\delta_{L T}=\epsilon_{L T} \frac{\sqrt{T_{3_{\text {Scaler }}-T_{3_{\text {File }}}}}}{T_{3_{\text {Scaler }}}}
$$

\subsection{Comparisons with Other Measurements}

Now that we have a value for our cross section with reasonable uncertainties we want to see how it compares to other measurements and fits. For these comparisons we will use the elastic ${ }^{3} \mathrm{He}$ cross section measurements from $[3$ as well as the final sum of Gaussians fit from [29]. In [3] there are several elastic cross section measurements of ${ }^{3} \mathrm{He}$ in similar kinematic regions to those of experiment E08-014. Let us examine the data point measured at the closest kinematic to experiment E08-014. This point was taken at $E_{0}=3.304 \mathrm{GeV}$ and a scattering angle of $20.83^{\circ}$ giving an elastic cross section of $1.57 \times 10^{-6} \mu \mathrm{b} / \mathrm{sr} \pm 0.10 \times 10^{-6} \mu \mathrm{b} / \mathrm{sr}$ at $Q^{2}=34.1 \mathrm{fm}^{-2}$.

The point found in this analysis is taken at $E_{0}=3.356 \mathrm{GeV}$ and a scattering angle of $20.51^{\circ}$ giving a cross section of $1.335 \times 10^{-6} \mu \mathrm{b} / \mathrm{sr} \pm 0.086 \times 10^{-6} \mu \mathrm{b} / \mathrm{sr}$ at $Q^{2}=$ $34.19 \mathrm{fm}^{-2}$. The error bars for these points almost overlap in spite of their slightly different kinematics. These two values can be said to be in reasonable agreement, especially considering that the $Q^{2}$ value for this experiment is larger. This implies that our extracted cross section should be smaller than the point from [3] which agrees with the data.

Next let us take the SOG form factor fits from [29] and calculate the cross section from these fits at our kinematics. The Amroun fit at $E_{0}=3.356 \mathrm{GeV}$ and a scattering angle of $20.51^{\circ}$ gives a cross section of $1.887 \times 10^{-6} \mu \mathrm{b} / \mathrm{sr}$. This is significantly different 
from the cross section derived in this thesis. However, the datasets from [29] had very little data in this high $Q^{2}$ region. Significantly more data in this high $Q^{2}$ region was added by [3] and [34] which were used in this analysis. This explains why the Amroun cross section prediction diverges from the cross section found in this analysis. Overall, the cross section found in this thesis seems to be in reasonable agreement with other measurements, and indicates that older fits may shift noticeably when the new higher $Q^{2}$ data is introduced. 


\section{Chapter 4}

\section{Global Fits}

This chapter will discuss the world data for ${ }^{3} \mathrm{H}$ and ${ }^{3} \mathrm{He}$ elastic cross sections. This data will then be fit using a sum of Gaussians (SOG) technique. These new global fits will incorporate modern datasets added to the world data since the last global fits were performed. This will include new high $Q^{2}$ data from JLab for ${ }^{3} \mathrm{He}$ as well as the ${ }^{3} \mathrm{He}$ cross section extracted in this thesis. The SOG fitting technique allows the electric and magnetic form factors to be easily extracted as well as for charge radii to be calculated. These new results will then be compared to past fits as well as some theory predictions.

\subsection{World Data}

The world data for ${ }^{3} \mathrm{H}$ and ${ }^{3} \mathrm{He}$ elastic cross sections spans 50 years and many different laboratories. Due to the expansiveness of the dataset there are many inconsistent methodologies employed in the different analyses collected. Efforts were taken to make these comparisons as consistent as reasonably possible, however it was often impossible with the existing literature to be certain which techniques were used. Methodological differences in modifications like radiative corrections and Dirac Wave Born Approximation (DWBA) techniques would be extremely time consuming to force all datasets into complete agreement. As such some of the datasets fit together are not 
completely apples-to-apples comparisons. Fortunately, the methodological differences result in very minor changes to the final cross sections, and thus do not significantly impact the efficacy of the new global fits.

Table 4.1 lists the literature comprising the current world data of ${ }^{3} \mathrm{H}$ elastic cross sections and Table 4.2 contains the ${ }^{3} \mathrm{He}$ world data compiled for this analysis. The table is organized chronologically from oldest dataset to most recent. The table lists the title of each publication, the first author listed on each publication, the journal the publication appeared in, and the location of the measurement with the year it was published. The table also contains physics data on each experiment. This physics data includes the rough $Q^{2}$ range covered by the experiment, whether the paper lists cross sections explicitly, whether the paper lists form factors explicitly, if the paper applied a phase shift correction to account for the plane wave approximation, and finally a brief note on the radiative corrections each paper used. Whenever a table entry wasn't listed or was unclear in the literature a '?' was used. 
Table 4.1: Accumulated World Data for ${ }^{3} \mathbf{H}$ Elastic Scattering

\begin{tabular}{|c|c|c|c|c|c|c|c|c|}
\hline Title & Authors & Journal & $\begin{array}{c}\text { Date/ } \\
\text { Location }\end{array}$ & $\begin{array}{c}Q^{2} \text { Range } \\
\left(\mathbf{f m}^{-2}\right)\end{array}$ & $\begin{array}{c}\text { Cross } \\
\text { Sections }\end{array}$ & $\begin{array}{c}\text { Form } \\
\text { Factors }\end{array}$ & $\begin{array}{c}\text { Phase } \\
\text { Shift }\end{array}$ & $\begin{array}{c}\text { Radiative } \\
\text { Corrections }\end{array}$ \\
\hline $\begin{array}{l}\text { Elastic Electron Scattering } \\
\text { from Tritium and Helium-3 }\end{array}$ & Collard & $\begin{array}{l}\text { Phys. Rev. } \\
\text { Vol. 138, No. 1B } 35]\end{array}$ & $\begin{array}{l}1965^{*} \\
\text { SLAC }\end{array}$ & $1-8$ & Yes & Yes & $?$ & Tsai \\
\hline $\begin{array}{l}\text { Triton Form Factor } \\
\text { from } 0.29-1.00 \mathrm{fm}^{-2}\end{array}$ & Beck & $\begin{array}{c}\text { Phys. Rev. C } \\
\text { Vol. 25, No. 3, 1152-1155 36] }\end{array}$ & $\begin{array}{c}1982 \\
\text { Saskatchewan }\end{array}$ & $0.29-1$ & Yes & $\begin{array}{c}\text { Yes } \\
\left(G_{E}\right)\end{array}$ & $?$ & $\begin{array}{l}\text { Meister } \\
\text { Yennie }\end{array}$ \\
\hline $\begin{array}{c}\text { Tritium Form Factors } \\
\text { at Low q }\end{array}$ & Beck & $\begin{array}{c}\text { Phys. Rev. C } \\
\text { Vol. 30, No. 5, 1403-1408 37] }\end{array}$ & $\begin{array}{c}1984^{*} \\
\text { NBS MIT }\end{array}$ & $0.05-3$ & Yes & Yes & $\begin{array}{c}\text { Yes } \\
\left(q_{e f f}\right)\end{array}$ & Mo/Tsai \\
\hline $\begin{array}{l}\text { Tritium Electromagnetic } \\
\text { Form Factors }\end{array}$ & Juster & $\begin{array}{l}\text { Phys. Rev. Letters } \\
\text { Vol. 55, No. 21, 2261-2264 38] }\end{array}$ & $\begin{array}{l}1985 \\
\text { Saclay }\end{array}$ & $0.3-31$ & $\begin{array}{l}\text { In Amroun } \\
1994\end{array}$ & $\begin{array}{c}\text { Yes } \\
(\mathrm{SOG})\end{array}$ & $?$ & Auffret \\
\hline $\begin{array}{l}\text { Isoscalar and Isovector Form } \\
\text { Factors of }{ }^{3} \mathrm{H} \text { and }{ }^{3} \mathrm{He} \text { for } \mathrm{Q} \\
\text { below } 2.9 \mathrm{fm}^{-1} \text { from Electron- } \\
\text { Scattering Measurements }\end{array}$ & Beck & $\begin{array}{l}\text { Phys. Rev. Letters } \\
\text { Vol. 59, No. 14, 1537-1540 } 39]\end{array}$ & $\begin{array}{l}1987 \\
\text { Bates }\end{array}$ & $0.03-9$ & No & $\begin{array}{l}\text { Yes } \\
\text { (Iso) }\end{array}$ & Yes & Mo/Tsai \\
\hline $\begin{array}{l}{ }^{3} \mathrm{H} \text { and }{ }^{3} \mathrm{He} \\
\text { Electromagnetic } \\
\text { Form Factors }\end{array}$ & Amroun & $\begin{array}{l}\text { Nuc. Phys. } \\
\text { A579 596-626 29] }\end{array}$ & $\begin{array}{l}1994^{*} \\
\text { Saclay }\end{array}$ & $1-47$ & Yes & Yes & Yes & $\begin{array}{c}\text { Mo/Tsai, Schwinger } \\
\text { and bremsstrahlung + } \\
\text { Landau Straggling }\end{array}$ \\
\hline
\end{tabular}


Table 4.2: Accumulated World Data for ${ }^{3}$ He Elastic Scattering

\begin{tabular}{|c|c|c|c|c|c|c|c|c|}
\hline Title & Authors & Journal & $\begin{array}{c}\text { Date/ } \\
\text { Location }\end{array}$ & $\begin{array}{c}Q^{2} \text { Range } \\
\left(\mathrm{fm}^{-2}\right)\end{array}$ & $\begin{array}{c}\text { Cross } \\
\text { Sections }\end{array}$ & $\begin{array}{l}\text { Form } \\
\text { Factors }\end{array}$ & $\begin{array}{c}\text { Phase } \\
\text { Shift }\end{array}$ & $\begin{array}{c}\text { Radiative } \\
\text { Corrections }\end{array}$ \\
\hline $\begin{array}{l}\text { Elastic Electron Scattering } \\
\text { from Tritium and Helium-3 }\end{array}$ & Collard & $\begin{array}{l}\text { Phys. Rev. } \\
\text { Vol. 138, No. 1B } 35]\end{array}$ & $\begin{array}{l}1965^{*} \\
\text { SLAC }\end{array}$ & $1-8$ & Yes & Yes & $?$ & Tsai \\
\hline $\begin{array}{c}\text { Elastic Electron Scattering } \\
\text { from }{ }^{3} \mathrm{He} \text { at High } \\
\text { Momentum Transfer }\end{array}$ & Bernheim & $\begin{array}{l}\text { Lettere Al Nuovo Cimento } \\
\text { Vol. 5, No. 5, 431-434 }\end{array}$ & $\begin{array}{l}1972 \\
\text { Orsay }\end{array}$ & $9-16$ & No & Yes & $?$ & "Usual" \\
\hline $\begin{array}{l}\text { Electromagnetic Structure } \\
\text { of the Helium Isotopes }\end{array}$ & McCarthy & $\begin{array}{c}\text { Phys. Rev. C } \\
\text { Vol. 15, No. 4, 1396-1414 41] }\end{array}$ & $\begin{array}{c}1977 \\
\text { Stanford HEPL }\end{array}$ & $0.3-20$ & No & Yes & Yes & Mo/Tsai \\
\hline $\begin{array}{c}\text { Low-Momentum-Transfer } \\
\text { Elastic Electron } \\
\text { Scattering from }{ }^{3} \mathrm{He}\end{array}$ & Szalata & $\begin{array}{c}\text { Phys. Rev. C } \\
\text { Vol. 15, No. 4, 1200-1203 } 42\end{array}$ & $\begin{array}{c}1977^{*} \\
\text { National Bureau } \\
\text { of Standards }\end{array}$ & $0.03-0.33$ & $\begin{array}{c}\text { Yes } \\
{ }^{3} \mathrm{He} /{ }^{12} \mathrm{C} \\
\text { Exp. }\end{array}$ & $\begin{array}{c}\text { Yes } \\
\left(F_{c h}^{2}\right)\end{array}$ & Yes & $\begin{array}{l}\text { "In the } \\
\text { Standard } \\
\text { Fashion" }\end{array}$ \\
\hline $\begin{array}{c}\text { Elastic Scattering } \\
\text { from }{ }^{3} \mathrm{He} \text { and }{ }^{4} \mathrm{He} \text { at } \\
\text { High Momentum Transfer }\end{array}$ & Arnold & $\begin{array}{l}\text { Phys. Rev. Letters } \\
\text { Vol. 40, No. } 22 \text { 43] }\end{array}$ & $\begin{array}{l}1978^{*} \\
\text { SLAC }\end{array}$ & $18-103$ & No & $\begin{array}{c}\text { Yes } \\
\left(A^{1 / 2}\right)\end{array}$ & $?$ & $?$ \\
\hline $\begin{array}{l}\text { Magnetic Form } \\
\text { Factor of }{ }^{3} \mathrm{He}\end{array}$ & Cavedon & $\begin{array}{l}\text { Phys. Rev. Letters } \\
\text { Vol. 49, No. 14, 986-989 44] }\end{array}$ & $\begin{array}{c}1982 \\
\text { Saclay }\end{array}$ & $7-32$ & $\begin{array}{c}\text { In Amroun } \\
1994\end{array}$ & $\begin{array}{c}\text { Yes } \\
\left(F_{M}^{2}\right)\end{array}$ & $\begin{array}{c}\text { Yes } \\
(\text { HADES) }\end{array}$ & Yes \\
\hline
\end{tabular}




\section{${ }^{3}$ He Magnetic}

Form Factor

Elastic Electron Scattering from ${ }^{3} \mathrm{He}$ and ${ }^{4} \mathrm{He}$

Isoscalar and Isovector Form Factors of ${ }^{3} \mathrm{H}$ and ${ }^{3} \mathrm{He}$ for $\mathrm{Q}$ below $2.9 \mathrm{fm}^{-1}$ from ElectronScattering Measurements

Isospin Separation of ThreeNucleon Form Factors

$$
{ }^{3} \mathrm{H} \text { and }{ }^{3} \mathrm{He}
$$

Electromagnetic Form Factors

Measurement of the Elastic Magnetic Form Factor of $3 \mathrm{He}$ at High Momentum Transfer JLab Measurements of the ${ }^{3}$ He Form Factors at Large

\section{Phys. Rev. C}

Dunn

Otterman

Beck

Phys. Rev. Letters

Vol. 59, No. 14, 1537-1540 39]

1987

Bates

Phys. Rev. Letters

Amroun

Vol. 69, No. 2, 253-256

Amroun

Nakagawa

Phys. Rev. Letters

Vol. 86, No. 24, 5446-5449 34]

Phys. Rev. Letters

Camsonne

$1992^{*}$

Saclay

$\begin{array}{cc}\text { Nuc. Phys. } & 1994^{*} \\ \text { A579 596-626 29] } & \text { Saclay }\end{array}$

$2001^{*}$

Bates

$2016^{*}$

JLab
0.08-11

Yes

Yes

Yes

Yes

Yes

$0.2-3.7$

$$
\text { No }
$$

(HADES)

Yes

0.03-9

$$
\text { No }
$$

$$
\text { (Iso) }
$$

Yes

$$
\text { Mo/Tsai }
$$

$2.6-37$

In Amroun

1994

No

Yes

"Standard"

Mo/Tsai, Schwinger

2-48 Yes $\quad$ Yes $\quad$ Yes

and bremsstrahlung +

Landau Straggling

Vol. 119, No. 162501, 1-6 3]
6-43

Yes

Yes

$\left(\left|F_{M}\right|^{2}\right)$

Yes

Mo/Tsai
Yes

Yes

$\left(q_{e f f}\right)$ 
The datasets used in the SOG global fits in this thesis are marked with a ${ }^{*}$ after the listed dates. The datasets that are new as of the previous fits in Amroun [29] are Camsonne [3], Nakagawa [34], and the cross section measurement from this analysis. Not all of the listed datasets could be used in this analysis for various reasons. The most common reason a dataset was not used was simply that the publication did not list its cross section data points explicitly in the publication so they could not be added to the fit. Another common reason was publications listing only the extracted form factors and not cross sections. This is not an issue when the publication also lists the beam energy and scattering angle for each data point (or $Q^{2}$ and one of either the energy or angle) as the cross section can be computed using these values. However, numerous publications list only the form factors without energies or angles making it impossible to calculate a cross section for each data point to be used in the global fit. Some publications like Arnold 1978 [43] used different ways to parametrize form factors, and whenever possible these methods were converted to cross sections.

\subsection{Sum of Gaussians Parametrization}

The sum of Gaussians (SOG) parametrization is a powerful method for fitting nuclear cross section data developed by Ingo Sick in the early 1970s [48]. It attempts to fit elastically scattered electron cross sections by representing the electric and magnetic charge densities as the sum of numerous Gaussians (see Figure 4.1). The Fourier transforms of these densities yield the electric form factor (sometimes referred to as the charge form factor, $\left.F_{c h}\right)$ and the magnetic form factor $\left(F_{m}\right)$ respectively. The technique attempts to remain model independent while taking several physical requirements for the form factors and nuclear wave functions in to account. However, a model dependence of sorts does enter the fits in the form of the radii at which the various Gaussians are situated. SOG fits make the extraction of the charge and magnetic form factors easy to extract, and along with them the charge density and charge radii of the target. 
Sick outlines the rules for removing a global model dependence when fitting cross section data as follows,

1. "Accept some clearly specified limitation to generality (accept some model dependence), since data with infinite $q_{\max }$ are not available (wavelengths smaller than $\lambda=\frac{2 \pi}{q_{\max }}$ are not determined by experiment).

2. Choose a restriction to generality which can be justified by physical arguments.

3. Write the density in a manner which decouples densities at different radii as much as possible." 48

One of the first physical restrictions that can be applied by the SOG parametrization is on the nuclear charge densities. No structures in the nuclear charge densities are allowed to be smaller than the RMS radii of the proton [48]. As this thesis is often using [29] as a point of comparison this work employs the same minimum size allowed for structure used by Amroun et al. 29 of $0.8 \mathrm{fm}$, or slightly less than the proton's radius.

Gaussians are used to build the structure of the fits since they fall off quickly enough so as to not strongly interfere with other Gaussians not nearby them, satisfying rule 2 above, while also being quite flexible when summed to mimic a distribution. Gaussians also work well with the rules and limitations imposed earlier. However, one should note that the Gaussians have no physical meaning themselves. One can write the nuclear charge density as shown in Equation 4.1. where the charge density is represented as a sum of numerous Gaussians set at different radii $R_{i}$ (an example is given later in this section and can be seen in Figure 4.1). The cross sections are then fitted using the $A_{i}$ as the free parameters. These Gaussians have their full width at half maximum restricted by the parameter $\gamma$ as required by the physical restrictions imposed above. The smallest width structure allowed is given by $\Gamma$ where $\Gamma=2 \gamma \sqrt{\ln (2)} 48$. 


$$
\rho(r) \propto \sum_{i=1}^{N} A_{i} e^{-\left(r-R_{i}\right)^{2} / \gamma^{2}}
$$

As previously mentioned, the $R_{i}$, representing the radii at which different Gaussians are located, form their own sort of model dependence. Since we are unable to study what happens above $q_{\max }$ the $R_{i}$ are analogous to a model of how the charge density behaves above $q_{\max }$. This issue can be resolved by choosing many different $R_{i}$ values randomly and fitting the $A_{i}$ to the data for each set of $R_{i}$ chosen randomly. The choice of $R_{i}$ may be random, but it does have numerous conditions applied. More will be said on the selection of the $R_{i}$ later in Section 4.3.1.

Once a large number of fits of the data using different sets of $R_{i}$ have been generated the 'good' fits must be distinguished from the 'bad'. This is done in several ways which will be discussed in more detail in Section 4.3.2, and include finding lower $\chi^{2}$ fits as well as making sure the fits' form factors appear physical. Once the 'good' fits are identified an uncertainty band can be built up by plotting each of the fits on top of one another. After a sufficient number of different 'good' $R_{i}$ sets have been fitted to the data the whole of the available model space has been explored.

The charge density is expected to have a derivative of zero at a $r=0$ [48]. This is not accounted for in Equation 4.1. To resolve this issue a tail can be added to each Gaussian that represents the Gaussian's behavior at $r<0$. This modified definition of the charge density is given in Equation 4.248 .

$$
\rho(r)=\frac{Z e}{2 \pi^{3 / 2} \gamma^{3}} \sum_{i=1}^{N} \frac{Q_{i}}{1+\frac{2 R_{i}^{2}}{\gamma^{2}}}\left(e^{-\left(r-R_{i}\right)^{2} / \gamma^{2}}+e^{-\left(r+R_{i}\right)^{2} / \gamma^{2}}\right)
$$

Equation 4.2 is normalized by Equation 4.3 . The $Q_{i}$ are now the parameters fitted to the data. The $Q_{i}$ are required to be positive as they represent the fraction of electric or magnetic charge carried by each Gaussian. $\sum Q_{i}=1$ is also required of the $Q_{i}$ terms as all of the charge fractions must sum to the total charge (one). Note that $\sum Q_{i}$ also equals the $Y$-intercept of the corresponding charge or magnetic form factor. $Z$ is the atomic number of the target and $e$ is the elementary charge [48]. 


$$
4 \pi \int_{0}^{\infty} \rho(r) r^{2} d r=Z e
$$

Figure 4.1 shows the charge density for ${ }^{3} \mathrm{He}$ along with the individual Gaussians from the sum of Gaussians in Equation 4.2. Summing each of these 12 Gaussians results in the charge density shown in the figure. The ${ }^{3} \mathrm{He}$ charge density shown in Figure 4.1 is derived from the ${ }^{3} \mathrm{He}$ representative form factor fits discussed in Section 4.3.3. Examining the first few Gaussians one finds that Gaussian 1 contains $35.7 \%$ of the charge density, Gaussian 2 contains $33.3 \%$ of the charge density, Gaussian 3 contains $1.9 \%$ of the charge density, and Gaussian 4 contains $15.1 \%$ of the charge density. In general the Gaussians centered at smaller radii hold a larger portion of the total charge density than those centered at larger radii with a few exceptions, like Gaussian 3.

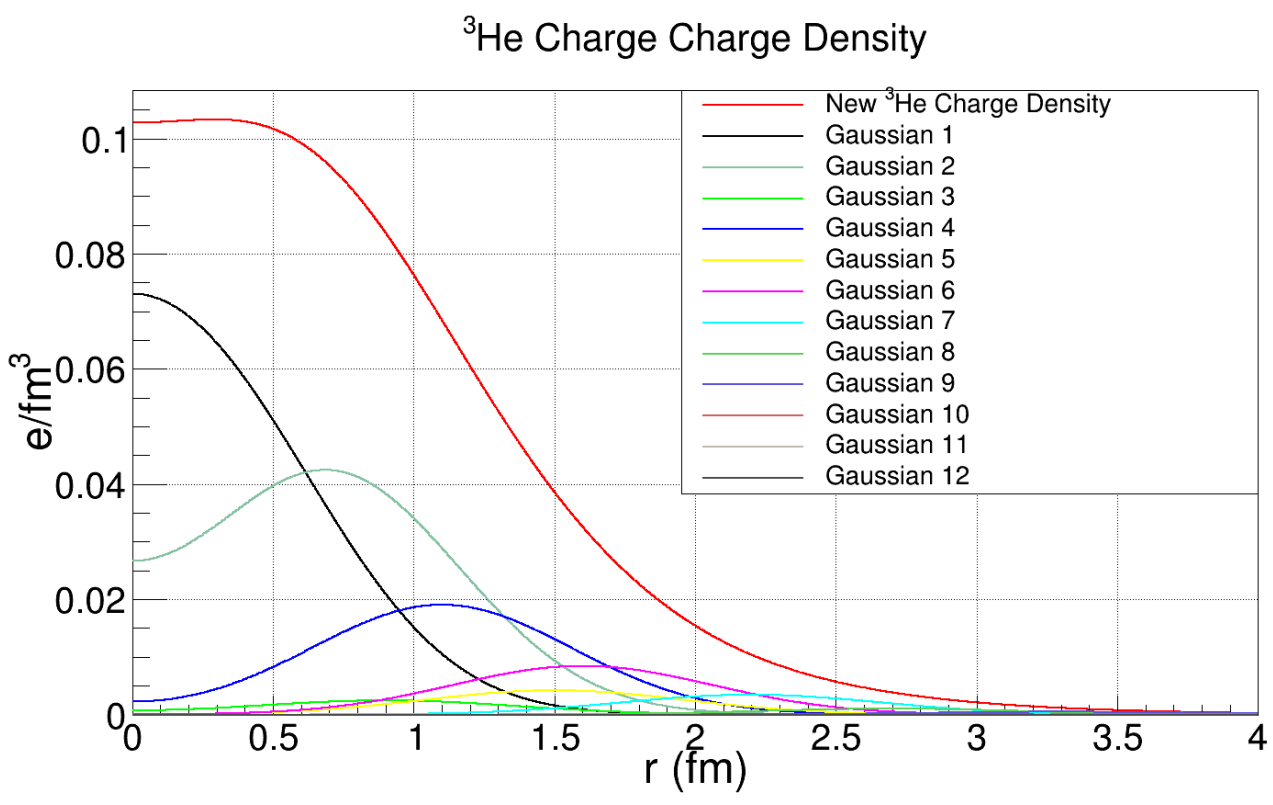

Figure 4.1: Individual Gaussians of the ${ }^{3} \mathbf{H e}$ Charge Density. The charge density for ${ }^{3} \mathrm{He}$ is plotted using the representative form factor fits from Section 4.3.3. Each individual Gaussian from Equation 4.2 is shown. Summing these individual Gaussians yields the ${ }^{3} \mathrm{He}$ charge density.

When using the plane wave Born approximation (PWBA) the electric and magnetic form factors can be parametrized as in Equation 4.4 48]. 


$$
F_{(c h, m)}(q)=\exp \left(-\frac{1}{4} q^{2} \gamma^{2}\right) \sum_{i=1}^{n} \frac{Q_{i(c h, m)}}{1+2 R_{i}^{2} / \gamma^{2}}\left(\cos \left(q R_{i}\right)+\frac{2 R_{i}^{2}}{\gamma^{2}} \frac{\sin \left(q R_{i}\right)}{q R_{i}}\right)
$$

At this point we will follow the procedure laid out in [29] and note that there is a typo in the reference. In 29] Equation (1) the $-1 / 2$ in the exponent should be a $-1 / 4$. Again the $Q_{i}$ are fitted to the data and represent the fraction of the electric or magnetic charge carried by each Gaussian. The $R_{i}$ are the radii at which the Gaussians are placed. $q$ is the four-momentum transferred via the virtual photon as discussed in Section 1.1. Lastly $\gamma$ is defined as $\gamma \sqrt{\frac{3}{2}}=0.8 \mathrm{fm}$ [29].

The cross section can be represented in PWBA with the SOG parametrization as shown in Equation 4.5 .

$$
\frac{d \sigma}{d \Omega}=\left(\frac{d \sigma}{d \Omega}\right)_{M o t t} \frac{1}{\eta}\left[\frac{Q^{2}}{\boldsymbol{q}^{2}} F_{c h}^{2}(q)+\frac{\mu^{2} Q^{2}}{2 M^{2}}\left(\frac{1}{2} \frac{Q^{2}}{\boldsymbol{q}^{2}}+\tan ^{2}\left(\frac{\theta}{2}\right)\right) F_{m}^{2}(q)\right]
$$

Here $\eta=1+Q^{2} / 4 M^{2}, Q^{2}$ is the squared four-momentum transfer from $1.3, \boldsymbol{q}^{2}$ is the three-momentum squared, $\mu$ is the magnetic moment of the target $\left(\mu_{3} \mathrm{He}=-\right.$ $2.1275 \times(3.0 / 2.0)$ and $\left.\mu_{3} H=2.9788 \times(3.0 / 1.0)\right)$, and $M$ is the mass of the target $\left(\mathrm{M}_{3} \mathrm{He}\right.$ $=3.0160293 \mathrm{amu}$ and $\mathrm{M}_{3}{ }_{H}=3.0160492 \mathrm{amu}$ ) [29].

The Mott cross section, $\left(\frac{d \sigma}{d \Omega}\right)_{M o t t}$, is given in Equation 1.25. Recall that $Z^{2}$ accounts for the charge of the target with $Z$ being the target's atomic number, $\frac{E^{\prime}}{E_{0}}$ is the recoil factor with $E_{0}$ being the scattered electron's initial energy and $E^{\prime}$ is the energy after scattering, $\alpha$ is the fine structure constant, and $\theta$ is the scattering angle. It is extremely important to be mindful of the units one is using when working with these equations. Be cautious of interchanging degrees and radians for the scattering angle, $\mathrm{fm}^{-2}$ and $\mathrm{GeV}^{2}$ for the squared four-momentum values, $\mathrm{fm}^{-1}$ and $\mathrm{GeV}$ for the energies, and amus and $\mathrm{GeV}$ for the mass units. Equation 4.6 shows the equivalent amount of $\mathrm{GeV}^{2}$ to $\mathrm{fm}^{-2}$ in nuclear units. 


$$
1 \mathrm{GeV}^{2} \approx 25.7 \mathrm{fm}^{-2}
$$

The assumption that the wave functions of the electrons are plane waves is not entirely correct. The nucleus' charge distorts these wave functions due to the Coulomb interaction, and thus shifts the $Q^{2}$ value to $Q_{\text {eff }}^{2}$ given in Equation 4.7. This leads to $Q_{e f f}^{2}$ taking the place of $Q^{2}$ in the above equations in this section (i.e. $Q^{2}$ is taken from the literature and then $Q_{e f f}^{2}$ is then calculated and used in the fits) 3 .

$$
Q_{e f f}^{2}=Q^{2}\left(1+\frac{1.5 Z \alpha}{E_{0} \times 1.12 \times A^{\frac{1}{3}}}\right)^{2}
$$

Here $A$ is the mass number and the other variables are defined above. The threemomentum, $\boldsymbol{q}^{2}$, is then given by Equation 4.8 where $\nu=E_{0}-E^{\prime}$ as in 1.2 .

$$
\boldsymbol{q}^{2}=\nu^{2}-Q_{e f f}^{2}
$$

\subsection{New SOG Fits}

The world data for ${ }^{3} \mathrm{H}$ and ${ }^{3} \mathrm{He}$ described in Section 4.1 will be fitted with the sum of Gaussians parametrization described in Section 4.2 in this section. This section will explain the choices made for each of the SOG fits such as the number of Gaussians used to fit the world data. It will also describe the choices made involving the $Q_{i}$ fit parameters. The placement and spacing of the $R_{i}$ radii at which the Gaussians are placed will also be discussed. A method used to try to optimize the fits by adjusting the $R_{i}$ spacing while attempting to minimize $\chi^{2}$ will be described.

\subsubsection{Gaussian Radii Placement}

As discussed in Section 4.2, the $R_{i}$ are the radii at which the SOG Gaussians are placed. This means that they represent a sort of model dependence. To explore all of the model space many different random $R_{i}$ combinations must be used to fit the world 
data. However, selecting the $R_{i}$ totally at random is extremely inefficient since we are only interested in $R_{i}$ combinations that yield reasonable fits and physical form factors.

To explore the $R_{i}$ combinations we want to apply a few rules to their selection. The first of which is that there is some radii, $R_{\max }$, beyond which the charge density has fallen almost to zero. Therefore, there is no reason to position any $R_{i}$ beyond $R_{\max }$. For ${ }^{3} \mathrm{H}$ and ${ }^{3} \mathrm{He} R_{\max }$ is $\approx 5 \mathrm{fm}$, although it is allowed to diverge from this radii as the fits are optimized. For the majority of fits, after the optimization procedure discussed below, $R_{\max }$ is found to be in the range of $4 \mathrm{fm}<R_{\max }<6 \mathrm{fm}$ centered around $5 \mathrm{fm}$.

Once a reasonable upper limit on the radii is established the spacing separating the $R_{i}$ from one another must be determined. It has been found that the spacing of the $R_{i}$ for $R_{i}<R_{\max } / 2$ should be approximately half as far apart as the $R_{i}$ spacing for the radii positioned at $R_{i}>R_{\max } / 2$ [48]. This is done so that the charge density region with more charge, i.e. closer to the nucleus, is described by more Gaussians. This allows the structure to be better captured by the SOG fits. Further away from the nucleus, where there is less charge, fewer Gaussians are needed to accurately describe the structure of the charge density.

Once the $R_{i}$ values are selected they are fitted using the SOG parametrization. Since we are interested in the fits that best describe the data it is logical to search for the lowest $\chi^{2}$ fits. We also want fits that produce form factors that have the physical properties we expect. This makes it is important to inspect the form factors visually for physicality. We define $\chi^{2}$ as in Equation 4.9 , where $N$ is the number of data points being fit, $\sigma_{\text {exp }}$ is the experimentally measured cross section at a particular $Q^{2}, \sigma_{f i t}$ is the cross section given by the global fit at the same $Q^{2}$ as the experimental cross section, and $\Delta$ is the total uncertainty attached to the experimental cross section at the given $Q^{2}$. (Note that all $\chi^{2}$ minimization fits in this analysis were performed using Minuit which can be found in 49.) A lower $\chi^{2}$ value naively indicates a better fit, but numerous flaws can occur when using only $\chi^{2}[50$. Numerous other tests for the 'goodness' of the fit were also applied to avoid this problem and are discussed in Section 4.3.2. 


$$
\chi^{2}=\sum_{n=1}^{N} \frac{\left(\sigma_{\text {exp }}-\sigma_{f i t}\right)^{2}}{\Delta^{2}}
$$

Initial $R_{i}$ spacings tend to be fairly unfavorable and produce large $\chi^{2}$ values and strangely shaped form factors. To minimize the $\chi^{2}$ as much as possible the $R_{i}$ values need to be allowed to shift. This is accomplished by first fitting the data with an initial set of $R_{i}$ values. After the initial fit is done each of the $R_{i}$ values is then optimized. If $R_{0}$ was initially $0.2 \mathrm{fm}$ the fit would then be redone with $R_{0}=0.1 \mathrm{fm}$ and then $R_{0}$ $=0.3 \mathrm{fm}$. The $R_{i}$ are each shifted up and down $0.1 \mathrm{fm}$ until $\chi^{2}$ gets larger. The $R_{i}$ that yielded the smallest $\chi^{2}$ is then kept as the 'optimal' $R_{i}$. Once this procedure is completed for each $R_{i}$ in ascending order the lowest, or at least close to the lowest, $\chi^{2}$ value for $R_{i}$ similar to the initial set of $R_{i}$ has been found.

As an example, let us examine the initial $R_{i}$ spacings for ${ }^{3} \mathrm{H}$ using eight Gaussians (see Figure 4.1 to see what the individual Gaussians look like). While the order of the Gaussians is irrelevant it is easier to code the $R_{i}$ in ascending radii length. Next we choose $R_{0}-R_{7}$ to meet the rules defined above. We want the sum of the spacings between the individual $R_{i}$ to be approximately $5 \mathrm{fm}$, and the $R_{i}$ spacing between consecutive Gaussians should be smaller at smaller radii. For ${ }^{3} \mathrm{H}$ with eight Gaussians the initial $R_{i}$ spacing is produced within given ranges randomly and then optimized as previously described. The ranges for the $R_{i}$ spacings are divided in steps of 0.1 $\mathrm{fm}$. The first Gaussian is placed near $\mathrm{R}=0 \mathrm{fm}$ and was chosen to be $R_{0}=0.2-0.3$ fm. This means that $R_{0}$ was randomly selected to initially be $0.2 \mathrm{fm}$ or $0.3 \mathrm{fm}$. Note that an $R_{0}$ of $0 \mathrm{fm}$ leads to poles in the parametrization. To avoid this issue a small number is used in place of 0 if $R_{0}=0 \mathrm{fm}$ is found to be the optimal radius.

After the first Gaussian is placed at $R_{0}$ Gaussians $R_{1-7}$ are placed by semi-randomly choosing their distance from the radii prior to them. The spacing for $R_{1-4}=0.5-0.6$ fm and for $R_{5-7}=0.8-0.9 \mathrm{fm}$ chosen randomly in the same manner as $R_{0}$. Notice that the radii further from the nucleus are placed approximately twice as far apart as the inner radii in accordance with the rules previously described. We can take the average 
spacing of each consecutive radius and sum them to find $R_{\max }$. Doing this we find $0.25 \mathrm{fm}+4 \times 0.55 \mathrm{fm}+3 \times 0.85 \mathrm{fm}=5 \mathrm{fm}$ which is the target $R_{\max }$. This process is then repeated for hundreds of semi-randomly generated $R_{i}$ sets which span the model space for ${ }^{3} \mathrm{H}$ and ${ }^{3} \mathrm{He}$.

\subsubsection{Number of Gaussians}

To utilize the SOG parametrization it is necessary to select the number of Gaussians, $N_{\text {Gaus }}$, to use for each fit. This process involves balancing several competing interests. If too few Gaussians are used the structure of the form factors may not be described in enough detail, but if too many Gaussians are used the data may be overfit. Overfitting would lead to the statistical noise in the data being mistaken for signal. The goal is then to fit the data as well as possible with no more parameters than required. A commonly used tool for selecting the best model is to calculate the $\chi^{2}$ value for a fit, however $\chi^{2}$ alone is insufficient and can often be deceptive and lead to issues such as overfitting [50].

To avoid this issue numerous other tests and metrics were applied when selecting $N_{\text {Gaus }}$ for ${ }^{3} \mathrm{H}$ and ${ }^{3} \mathrm{He}$. Among these are the $\chi^{2}$ value, the reduced $\chi^{2}$ value, Bayesian information criterion, Akaike information criterion, the sums of the fractions of the electric and magnetic charges held by the Gaussians, the percentage of fits that were deemed 'good', and finally a visual inspection of the form factors for known physical characteristics [50]. By combining these different tests it is possible to determine the number of Gaussians that provide an optimal fit. Note that it is not uncommon for two consecutive numbers of Gaussians to yield reasonably similar fits.

Reduced $\chi^{2}$, or $\chi_{r}^{2}$, is similar to $\chi^{2}$ from Equation 4.9 except that it takes the number of data points and the number of parameters used in the fit in to account. The equation for reduced $\chi^{2}$ used in this analysis is given in Equation 4.10, where $\chi^{2}$ is from 4.9. $N$ is the number of data points in the fit, and $N_{v a r}$ is the number of free parameters, or variables, used in the fit. Note that while $\chi^{2}$ must always decrease with the number of parameters added $\chi_{r}^{2}$ can increase if too many parameters have been 
added. This makes finding the fits with the lowest $\chi_{r}^{2}$ an elementary, but still useful, test that the proper number of parameters are being use to describe the data.

$$
\chi_{r}^{2}=\frac{\chi^{2}}{N-N_{v a r}-1}
$$

The next two tests applied to determine the number of Gaussians to use in the SOG fits are Akaike information criterion (AIC) defined in Equation 4.11 [51] and Bayesian information criterion (BIC) defined in Equation 4.12 [52] [50]. AIC and BIC are both a more advanced type of statistical test useful for selecting the proper model to use. The primary difference between the two is that BIC applies a larger penalty based on the number of model parameters used to fit the data. The way to select the correct model is to find the lowest $\mathrm{AIC}$ and $\mathrm{BIC}$ values, while remembering that these tests may choose slightly different models than the other tests and each other. To determine how much more evidence there is for one model versus another we can look at the difference between their BIC values, $\triangle \mathrm{BIC}$. A $\Delta \mathrm{BIC}$ of $0<\Delta \mathrm{BIC}<2$ indicates no real difference between models, $2<\Delta \mathrm{BIC}<6$ indicates that there is positive evidence for the lower valued model, $6<\Delta \mathrm{BIC}<10$ indicates strong evidence for the lower valued model, and $\triangle \mathrm{BIC}>10$ indicates very strong evidence for the lower valued model 53 .

$$
\begin{gathered}
A I C=N \ln \left(\frac{\chi^{2}}{N}\right)+2 N_{v a r} \\
B I C=N \ln \left(\frac{\chi^{2}}{N}\right)+\ln (N) N_{v a r}
\end{gathered}
$$

When selecting the number of Gaussians to use the sum of the electric and magnetic charges is also examined. The sum of the $Q_{i}$ charges should sum to a charge of unity, however the fits do not enforce this requirement. Instead the sum of the $Q_{i}$ are allowed to fluctuate with the best fit values of the individual $Q_{i}$. This then makes the sums another sort of test of the goodness of each fit. A 'better' fit, or one that complies 
more with our predetermined knowledge of the form factors, will have $Q_{i}$ sums closer to unity. Values further from unity can indicate a worse fit, but they also help to indicate where more data is needed.

Finally a visual inspection of the form factors is applied. It is known that the form factors should have sharp minima as discussed in Section 1.3. Often the fits will have only a dip in the form factor where a sharp minimum should exist and can thus be discarded. An example of this is seen in Figure 4.2a. These nonphysical 'dip only' fits tend to have higher $\chi^{2}$ values so cutting on $\chi^{2}$ can generally eliminate them. More specifically, this is done by plotting the charge form factor, $F_{c h}$, and lowering the $\chi^{2}$ cut until all of the 'dip only' minima fits are removed leaving only the sharp minima expected. These remaining fits are deemed to be the 'good' fits. This process is done with the charge form factor as we have better data there. This procedure generally improves the corresponding magnetic form factors as well, but the lack of high $Q^{2}$ data for $F_{m}$ leads to more nonphysical or odd fits of $F_{m}$.

When fitting with any number of Gaussians many of the resulting fits do not meet the definition of a 'good' fit described above. The ratio of the 'good' fits to total fits attempted is representative of the likelihood of fits of $N_{\text {Gaus }}$ to converge to physical looking fits. Assume $N_{\text {Gaus }}=9$ gives a 'good' fit $40 \%$ of the time, and $N_{\text {Gaus }}=8$ gives a 'good' fit $5 \%$ of the time and has a slightly lower average BIC than $N_{\text {Gaus }}$ $=9$. This analysis takes the low convergence rate as evidence against the slightly lower BIC results and may favor the marginally higher BIC results due to their better convergence rate assuming $\triangle \mathrm{BIC}$ between the two average $\mathrm{BIC}$ values is small.

Previous analyses have also done a good job locating the first diffractive minima of the form factors and can be used to check the reasonableness of this analysis' fits. For example [29] locates the first minima of both ${ }^{3} \mathrm{H}$ and ${ }^{3} \mathrm{He}$, and [3] locates the minima for ${ }^{3} \mathrm{He}$. If this analysis' results diverge significantly in the previously well understood regions that is taken to be a strike against the model selected. Note that some movement in the ${ }^{3} \mathrm{He}$ magnetic form factor is not unexpected since new high $Q^{2}$ data is being incorporated into this analysis. 
Now that we have established the tools with which to select a model, let us determine how many Gaussians to use when fitting ${ }^{3} \mathrm{H}$ and ${ }^{3} \mathrm{He} .{ }^{3} \mathrm{He}$ will be examined first due to there being more, and often higher quality, data for ${ }^{3} \mathrm{He}$ than ${ }^{3} \mathrm{H}$. The method used to determine the number of Gaussians (i.e. the model) to use to fit the data was to run 100 fits of the ${ }^{3} \mathrm{He}$ world data for each reasonable value of $N_{\text {Gaus }}$. Then the various tests and metrics laid out above were computed for that value of $N_{\text {Gaus. }}$. These results were then compared and the 'optimal' number of Gaussians was determined.

Table 4.3 shows the results of this model selection analysis for ${ }^{3} \mathrm{He}$. All of the values in the table are averages of the surviving 'good' fits and the best values are bolded as is the final selection for $N_{\text {Gaus }} \cdot \chi_{\max }^{2}$ is the maximum $\chi^{2}$ cut that removed all of the nonphysical dip minima in the charge form factor, and 'Good Fits' is the number of the 100 fits that survived this cut. Note that not all of these factors are weighted equally. The highest preference is given to BIC and AIC followed by $\chi_{r}^{2}$ and visually inspecting the form factors. The other factors add more detailed information and are used more as tiebreakers and to raise red flags if something major is wrong.

\begin{tabular}{|ccccccccc|}
\hline $\boldsymbol{N}_{\text {Gaus }}$ & Avg. $\chi^{\mathbf{2}}$ & $\chi_{\boldsymbol{r}}^{\mathbf{2}}$ & BIC & AIC & $\sum \boldsymbol{Q}_{\boldsymbol{i}_{c h}}$ & $\sum \boldsymbol{Q}_{\boldsymbol{i}_{\boldsymbol{m}}}$ & $\boldsymbol{\chi}_{\text {max }}^{\mathbf{2}}$ & $\begin{array}{c}\text { 'Good' } \\
\text { Fits }\end{array}$ \\
\hline 8 & 584.9 & 2.417 & 255.4 & 223.2 & $\mathbf{1 . 0 0 8}$ & 1.111 & 765 & 11 \\
9 & 470.4 & 1.960 & 204.6 & 172.4 & 1.009 & $\mathbf{1 . 0 2 1}$ & 521 & 58 \\
10 & 469.2 & 1.971 & 209.5 & 173.8 & $\mathbf{1 . 0 0 8}$ & 1.082 & 519 & 66 \\
11 & 445.1 & 1.886 & $\mathbf{2 0 1 . 4}$ & 162.2 & $\mathbf{1 . 0 0 8}$ & 1.040 & 503 & 67 \\
$\mathbf{1 2}$ & $\mathbf{4 3 6 . 3}$ & $\mathbf{1 . 8 6 4}$ & 201.7 & $\mathbf{1 5 9 . 0}$ & $\mathbf{1 . 0 0 8}$ & 1.026 & 501 & $\mathbf{7 5}$ \\
13 & 439.1 & 1.893 & 208.9 & 162.7 & 1.009 & 1.040 & 500 & 56 \\
\hline
\end{tabular}

Table 4.3: Determination of $\boldsymbol{N}_{\text {Gaus }}$ for ${ }^{3} \mathbf{H e}$. Statistical values used to determine the optimal $N_{\text {Gaus }}$ to use for the SOG fits from an initial sample of 100 fits for each value $N_{\text {Gaus }}$. $N_{\text {Gaus }}$ is the number of Gaussians used in the SOG fits, Avg. $\chi^{2}$ is the average $\chi^{2}$ value for the fits, $\chi_{r}^{2}$ is the average reduced $\chi^{2}$ value for the fits, BIC is the average Bayesian information criterion value of the fits, AIC is the average Akaike information criterion value of the fits, $\sum Q_{i_{c h}}$ is the average sum of the $Q_{i_{c h}}$ fit parameters, $\sum Q_{i_{m}}$ is the average sum of the $Q_{i_{m}}$ fit parameters, $\chi_{\text {max }}^{2}$ is the maximum $\chi^{2}$ value for a fit to be deemed 'good', and 'Good Fits' is the number of fits out of 100 that converged to 'goodness'. The optimal value for each variable is bolded. 
Examining Table 4.3 it is clear that no model had the best value in every category so some further analysis is required to select $N_{\text {Gaus }}$ for ${ }^{3} \mathrm{He} . N_{\text {Gaus }}=12$ has the best value in both $\chi_{r}^{2}$ and $\mathrm{AIC}$ which are both important metrics. Examining the BIC it is seen that $N_{\text {Gaus }}=12$ and $N_{\text {Gaus }}=11$ have nearly identical BIC values. In fact $\Delta \mathrm{BIC}<0.4$ which indicates a negligible preference between the models. All of the $\sum Q_{i_{c h}}$ values are fairly close, offering little insight. The $\sum Q_{i_{m}}$ value for $N_{\text {Gaus }}=$ 12 is on the better end of the spectrum as well. $N_{\text {Gaus }}=12$ also had the most fits converge to be designated 'good' fits with charge form factors that appear physical. $N_{\text {Gaus }}=12$ further had the lowest average $\chi^{2}$ value, but this metric can be misleading as $\chi^{2}$ must always decrease as the number of parameters increases. However, because of the different $R_{i}$ configurations and averaging the $\chi^{2}$ results for the fits the average $\chi^{2}$ shown in the table does not always have to decrease with increasing $N_{\text {Gaus }}$. Upon reviewing these metrics, it is fairly clear the $N_{\text {Gaus }}=12$ is the best model to use for fitting the ${ }^{3} \mathrm{He}$ data.

Table 4.4 mirrors Table 4.3 and shows the results of this model selection analysis for ${ }^{3} \mathrm{H}$. It is immediately obvious that the $\chi^{2}$ values are larger for ${ }^{3} \mathrm{H}$ than they were for ${ }^{3} \mathrm{He}$. This is because the world data for ${ }^{3} \mathrm{H}$ is less complete than that of ${ }^{3} \mathrm{He}$, especially at higher $Q^{2}$, and the quality of the data is not as good as that of ${ }^{3} \mathrm{He}$. The $\sum Q_{i}$ values are also further from unity with the magnetic charges being especially far off. Once again, this is a product of the dearth of high $Q^{2}$ and back angle data in the world data. If more of this data could be obtained for ${ }^{3} \mathrm{H}$ the reduced $\chi^{2}$ of the fits would likely decrease. The poor $\sum Q_{i_{m}}$ agreement with the expectation of unity also demonstrates the analysis value of not forcing the $Q_{i}$ to sum to unity which could hide the need for more high $Q^{2}$ and back angle data.

There are two entries for $N_{\text {Gaus }}=8$ labelled ' 8 close' and ' 8 wide'. These refer to the initial spacing of the $R_{i}$ values. For the close entry $R_{0}=0.2-0.3, R_{1-4}=0.3-0.4$, and $R_{5-7}=0.5-0.6$, and for the wide entry $R_{0}=0.2-0.3, R_{1-4}=0.5-0.6$, and $R_{5-7}=$ 0.8-0.9 as explained in Section 4.3.1. This meant for the close $R_{i}$ the average starting $R_{\max }=3.3 \mathrm{fm}$ and for the wide spacing $R_{\max }=5 \mathrm{fm}$ which is what we expect from 
previous analyses [29]. This test was done to see if the final fit results depended strongly on the starting $R_{i}$ spacing, or if the $R_{i}$ optimization produced consistent results with less reasonable initial $R_{i}$ values.

Fortunately, the results for the closer and wider $R_{i}$ spacings come out very similar indicating that the initial choice of $R_{i}$ does not significantly change the final result. This test had also previously been done for ${ }^{3} \mathrm{He}$ with $N_{\text {Gaus }}=10$ with similar results to ${ }^{3} \mathrm{H}$ with $N_{\text {Gaus }}=8$. The major difference between the initial spacings was that the closer, less reasonable, $R_{i}$ took longer for the $R_{i}$ optimization code to process. This was because the code had to check more values for each $R_{i}$ before finding similar optimal values to the larger initial $R_{i}$ spacings. The close $R_{i}$ also had fewer fits converge to be designated 'good' fits indicating that more of the initial $R_{i}$ were unfavorable models than the wider spacings. We can conclude that if the initial $R_{i}$ distributions are off the final fit results should generally still be reliable, but they may take longer to process and have fewer fits converge to be deemed 'good'.

\begin{tabular}{|ccccccccc|}
\hline $\boldsymbol{N}_{\text {Gaus }}$ & Avg. $\chi^{\mathbf{2}}$ & $\boldsymbol{\chi}_{\boldsymbol{r}}^{\mathbf{2}}$ & BIC & AIC & $\sum \boldsymbol{Q}_{\boldsymbol{i}_{\text {ch }}}$ & $\sum \boldsymbol{Q}_{\boldsymbol{i}_{\boldsymbol{m}}}$ & $\boldsymbol{\chi}_{\boldsymbol{m a x}}^{\mathbf{2}}$ & $\begin{array}{c}\text { 'Good' } \\
\text { Fits }\end{array}$ \\
\hline 7 & 611.7 & 2.793 & $\mathbf{2 6 3 . 0}$ & 238.9 & $\mathbf{1 . 0 8 4}$ & 1.327 & 611.7 & 1 \\
8 close & 601.8 & $\mathbf{2 . 7 7 3}$ & 264.7 & 237.1 & 1.090 & 1.329 & 603 & 32 \\
$\mathbf{8}$ wide & 601.8 & 2.799 & 264.7 & $\mathbf{2 3 7 . 0}$ & 1.090 & 1.333 & 603 & 39 \\
9 & 601.8 & 2.826 & 270.1 & 239.0 & 1.088 & 1.320 & 604 & $\mathbf{9 5}$ \\
10 & 601.9 & 2.844 & 275.6 & 241.0 & 1.092 & $\mathbf{1 . 2 9 6}$ & 603 & 78 \\
11 & $\mathbf{6 0 0 . 8}$ & $\mathbf{2 . 7 7 3}$ & 280.6 & 242.6 & 1.087 & 1.341 & 602 & 88 \\
\hline
\end{tabular}

Table 4.4: Determination of $\boldsymbol{N}_{\text {Gaus }}$ for ${ }^{3} \mathbf{H}$. Statistical values used to determine the optimal $N_{\text {Gaus }}$ to use for the SOG fits from an initial sample of 100 fits for each value $N_{\text {Gaus }} . N_{\text {Gaus }}$ is the number of Gaussians used in the SOG fits, Avg. $\chi^{2}$ is the average $\chi^{2}$ value for the fits, $\chi_{r}^{2}$ is the average reduced $\chi^{2}$ value for the fits, BIC is the average Bayesian information criterion value of the fits, AIC is the average Akaike information criterion value of the fits, $\sum Q_{i_{c h}}$ is the average sum of the $Q_{i_{c h}}$ fit parameters, $\sum Q_{i_{m}}$ is the average sum of the $Q_{i_{m}}$ fit parameters, $\chi_{\max }^{2}$ is the maximum $\chi^{2}$ value for a fit to be deemed 'good', and 'Good Fits' is the number of fits out of 100 that converged to 'goodness'. The optimal value for each variable is bolded.

Again, the agreement between the metrics is not unanimous, and in fact it is even less clear than for ${ }^{3} \mathrm{He}$. Let us begin by examining the lowest BIC value for $N_{\text {Gaus }}$ 
$=7$. The other metrics also look decent until one notices that only one fit met the standards for a 'good' fit. This failure for the vast majority of fits to look physical indicates that $N_{\text {Gaus }}=7$ is probably not a good choice for the best model. Examining the lowest AIC value for the wider $R_{i}$ spacings and $N_{\text {Gaus }}=8$ the other metrics look acceptable with a reasonable number of fits converging to 'goodness'. $\triangle \mathrm{BIC}$ for the wider $R_{i}$ spacings and $N_{\text {Gaus }}=8$ compared to $N_{\text {Gaus }}=7$ is only 1.6 indicating that there is little reason to prefer one model over the other. The higher Gaussian fits look reasonable as well, but the AIC, and especially BIC, grow significantly as $N_{\text {Gaus }}$ increases ruling out these fits. The closer spacing for $N_{\text {Gaus }}=8$ was eliminated for the reasons discussed above like taking longer to process and having fewer fits converge but yielding similar results to the wider spaced $R_{i}$ models. Accounting for all of this we select $N_{\text {Gaus }}=8$ with the wider initial $R_{i}$ spacings.

\subsection{3 $\quad{ }^{3}$ He Fits}

Now that we have selected $N_{\text {Gaus }}=12$ for ${ }^{3} \mathrm{He}$ we can run several hundred fits with pseudorandom starting $R_{i}$ values along with the $R_{i}$ optimization procedure. The initial spacing of the $R_{i}$ values for these fits was $R_{0}=0.1-0.2, R_{1-6}=0.3-0.4$, and $R_{7-11}=0.5-0.6$ as explained in Section 4.3.1. A total of 1352 individual fits using the pseudorandom $R_{i}$ values were generated. Table 4.5 shows the results of these 1352 fits without any $\chi_{\max }^{2}$ cut and with a $\chi_{\max }^{2}=500$ in the same fashion as Tables 4.3 and 4.4. This $\chi^{2}$ cut was determined by decreasing the value of the cut until all of the $\mathrm{F}_{c h}$ form factors had nicely defined sharp first minima and the unphysical form factors were eliminated as discussed in Section 4.3.2, 852 fits survive the $\chi^{2}$ cut of 500, and the remaining fits have charge form factors with the desired sharp minima.

Table 4.5 shows that the $\chi_{\max }^{2}$ cut improves all of the metrics as expected. Of the 1352 fits $852,63 \%$, survive the $\chi_{\max }^{2}$ cut showing that the fits are not struggling to converge to 'goodness'. There are 259 data points for ${ }^{3} \mathrm{He}$ resulting in a $\chi^{2}$ of 436.6. This works out to a $\chi^{2}$ of 1.686 per data point. This value indicates a reasonably 


\begin{tabular}{|ccccccccc|}
\hline $\boldsymbol{N}_{\text {Gaus }}$ & Avg. $\chi^{\mathbf{2}}$ & $\chi_{\boldsymbol{r}}^{\mathbf{2}}$ & BIC & AIC & $\sum \boldsymbol{Q}_{\boldsymbol{i}_{\text {ch }}}$ & $\sum \boldsymbol{Q}_{\boldsymbol{i}_{\boldsymbol{m}}}$ & $\boldsymbol{\chi}_{\text {max }}^{\mathbf{2}}$ & $\begin{array}{c}\text { Below } \\
\text { Cut }\end{array}$ \\
\hline 12 & 523.7 & 2.238 & 249.1 & 184.8 & 1.010 & 1.046 & No Cut & 1352 \\
12 & 436.6 & 1.866 & 201.9 & 159.2 & 1.008 & 1.022 & 500 & 852 \\
\hline
\end{tabular}

Table 4.5: Metrics for Final ${ }^{3}$ He Fits. $N_{\text {Gaus }}$ is the number of Gaussians used in the SOG fits, Avg. $\chi^{2}$ is the average $\chi^{2}$ value for the fits, $\chi_{r}^{2}$ is the average reduced $\chi^{2}$ value for the fits, BIC is the average Bayesian information criterion value of the fits, AIC is the average Akaike information criterion value of the fits, $\sum Q_{i_{c h}}$ is the average sum of the $Q_{i_{c h}}$ fit parameters, $\sum Q_{i_{m}}$ is the average sum of the $Q_{i_{m}}$ fit parameters, $\chi_{\max }^{2}$ is the maximum $\chi^{2}$ value for a fit to be deemed 'good', and 'Below Cut' is the number of fits that survived the $\chi_{\max }^{2}$ cut.

good fit, but could be decreased if the individual datasets were each given a floating normalization. (A floating normalization would scale the results of each individual dataset by a constant so as to account for systematic differences between the individual datasets. For example, if one dataset's average results were consistently higher than the average of the other datasets' results by $10 \%$, then the first dataset would be scaled down by a constant so that its average would agree with the average of the other datasets. This procedure requires that the average of the majority be 'correct', i.e. that it approaches the true underlying function being measured, otherwise the data is being moved towards the wrong central value. It would be a simple matter to determine each dataset's normalization if the true function were known, but the purpose of these measurements is to find the true function.) This analysis chose not to apply floating normalizations to the individual datasets so as to better represent the current state of the world data and its uncertainties and disagreements.

The $\sum Q_{i_{c h}}=1.008$ when we expect it to equal unity from physical considerations of the form factors discussed in Section 1.3 . This means the fits on average see $0.8 \%$ more electric charge than expected. This occurs because we did not force the form factors to approach unity at the origin. Again, we hope to represent the world data as is and use the $\sum Q_{i}$ to indicate the quality and completeness of the available data. If we forced the form factors to unity at the origin their slope would be artificially decreased in magnitude near zero. Still, $\sum Q_{i_{c h}}=1.008$ is close to one and indicates that the world data describes the charge form factor for ${ }^{3} \mathrm{He}$ well. $\sum Q_{i_{m}}=1.022$ 
shows an excess of $2.2 \%$ in the magnetic charge which is worse than the electric charge due again to the lack of high $Q^{2}$ data. Even so, the $\sum Q_{i_{m}}$ for ${ }^{3} \mathrm{He}$ from the fits seems to correspond decently well with our prior expectations.

Now let's examine the fits from Table 4.5 visually. Figure 4.2 shows the resulting charge form factors of each of the 1352 fits of the ${ }^{3} \mathrm{He}$ world data. Figure $4.2 \mathrm{a}$ shows the 1352 fits without any $\chi_{\max }^{2}$ cut, and Figure $4.2 \mathrm{~b}$ shows the 852 fits surviving a $\chi_{\max }^{2}=500$ cut. Plotted along with this analysis' fits in red is a blue line representing the average fit result from 29 in the range that analysis considered its fits to be valid.

Examining the 1352 fits with no $\chi_{\max }^{2}$ cut in Figure $4.2 \mathrm{a}$ the first feature to notice is that many of the fits are behaving in an unphysical manner. We observe numerous 'dip only' minima where the first sharp minima is expected. Numerous fits also have first or second minima located in the already well understood region of $20-30 \mathrm{fm}^{-2}$ where there are not expected to be minima. We want to eliminate these kinds of fits as we know them to be nonphysical from theory and prior measurements.

To eliminate these fits we can impose a cut, $\chi_{\max }^{2}$, which removes fits with higher $\chi^{2}$ as described in Section 4.3.2. Removing higher $\chi^{2}$ fits will generally remove the worse fits first, hopefully eliminating the least physical looking results. A cut of $\chi_{\max }^{2}$ $=500$ on these 1352 fits results in 852 fits surviving and is shown in the plot in Figure $4.2 \mathrm{~b}$. By lowing $\chi_{\max }^{2}$ until the last of the 'dip only' minima were removed we have eliminated the nonphysical fits leaving only the desired 'good' fits. Now it is clear that our results for the ${ }^{3} \mathrm{He}$ charge form factor are in excellent agreement with [29]. No changes were expected in the charge form factor due to the addition of more high $Q^{2}$ data as the charge form factor is dominated by the low $Q^{2}$ data which was already robust. If the new high $Q^{2}$ data is going to change our understanding of a form factor it is far more likely to be the magnetic form factor.

Figure 4.3 shows the resulting magnetic form factors of each of the 1352 fits of the ${ }^{3} \mathrm{He}$ world data. Figure $4.3 \mathrm{a}$ shows the 1352 fits without any $\chi_{\max }^{2}$ cut plotted together, and Figure $4.3 \mathrm{~b}$ shows the 852 fits surviving the $\chi_{\max }^{2}=500$ cut. Beginning with the fits without a $\chi_{\max }^{2}$ cut from Figure $4.3 \mathrm{a}$ there are many unphysical fits again. 


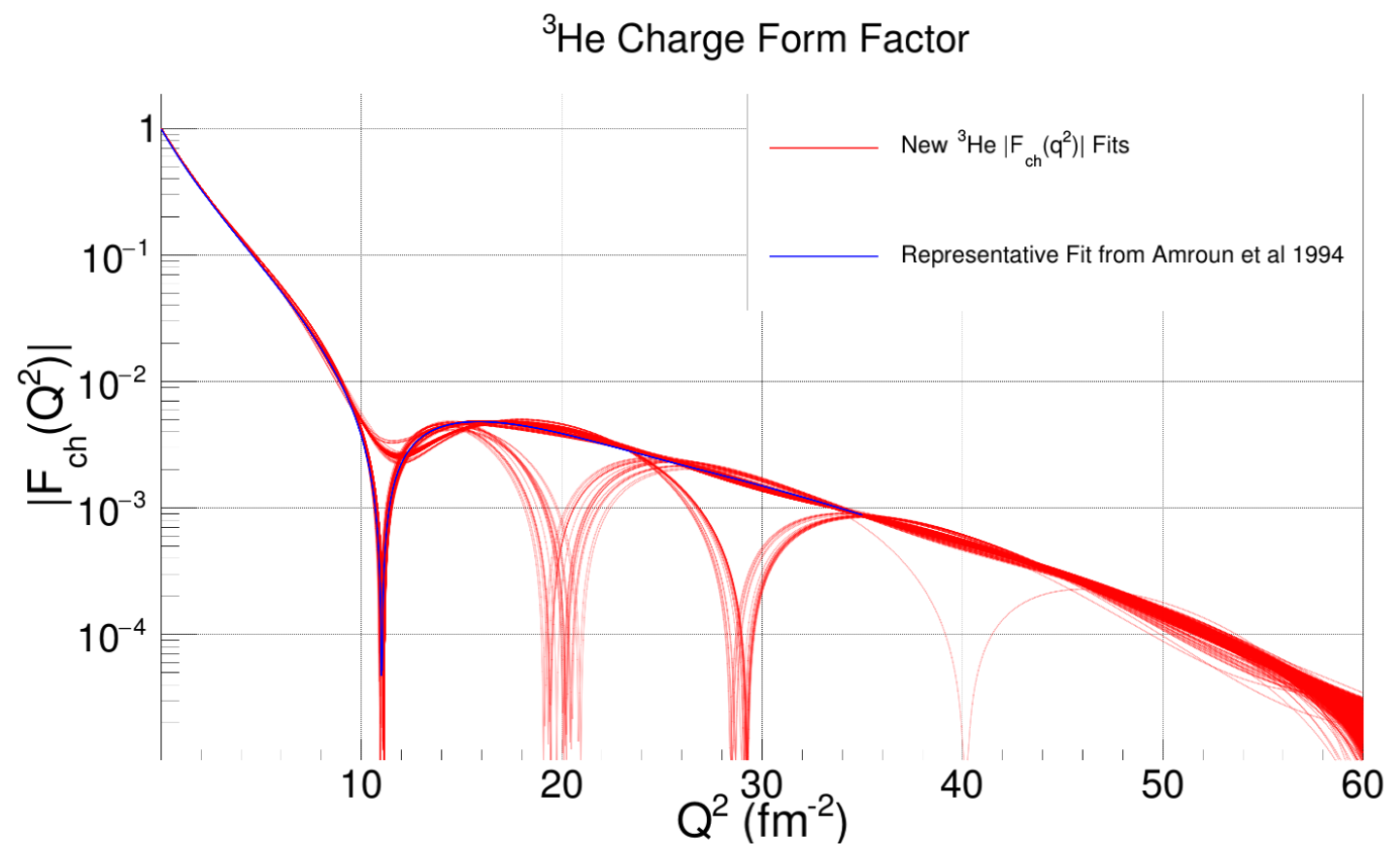

(a) Charge Form Factors from $1352{ }^{3} \mathrm{He}$ Fits with no $\chi_{\max }^{2}$ cut.

${ }^{3} \mathrm{He}$ Charge Form Factor

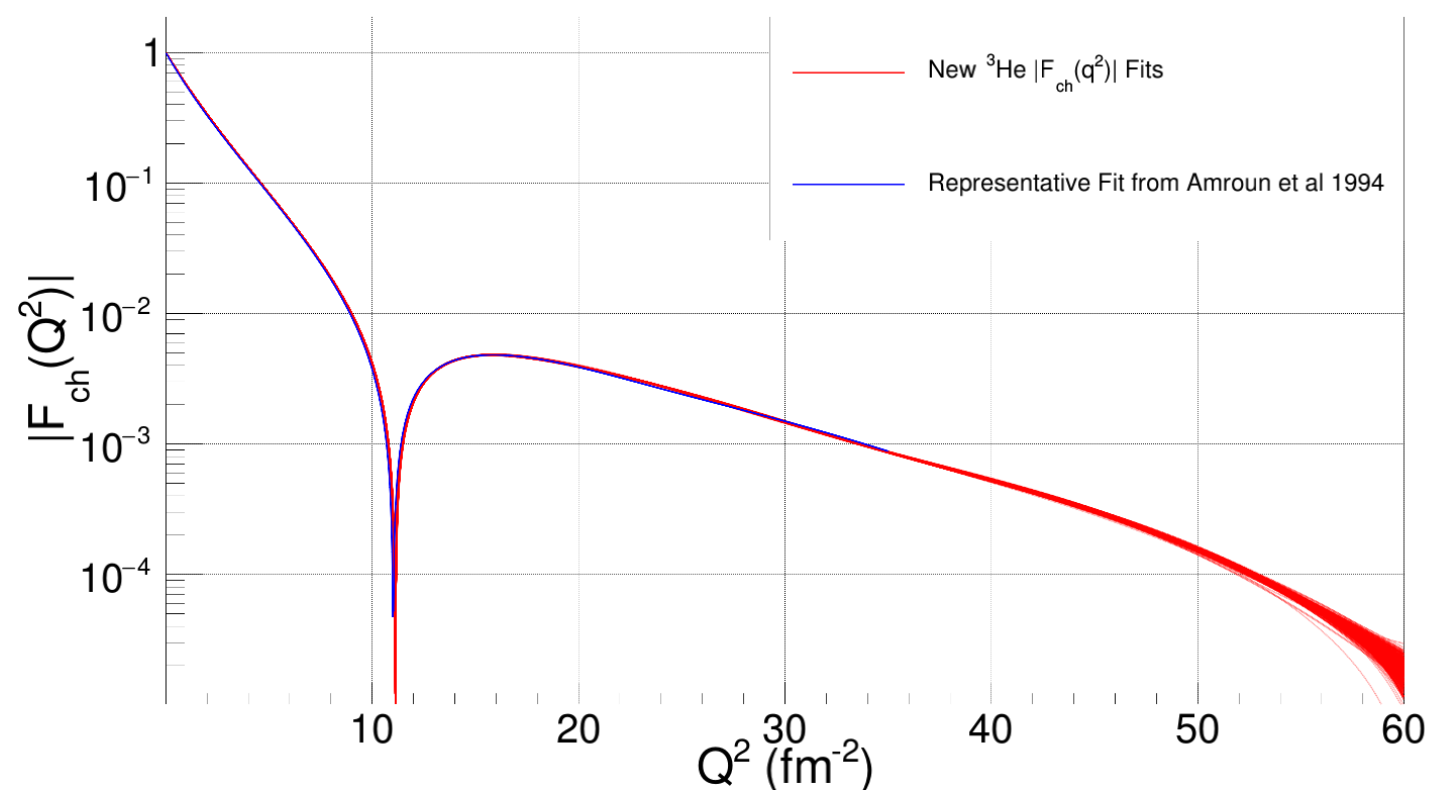

(b) Charge Form Factors from $852{ }^{3} \mathrm{He}$ Fits surviving a $\chi_{\max }^{2}=500$ cut.

Figure 4.2: ${ }^{3}$ He Charge Form Factors. These charge form factors (red lines) were derived from 1352 fits of the ${ }^{3} \mathrm{He}$ world data using pseudorandom initial $R_{i}$ values and the $R_{i}$ optimization procedure. The blue line is the fit from [29]. 
Most of these are identified by the location of their first minima being unreasonable, or by the minima not being sharp. It is expected that the magnetic form factor fits will be less clean than the charge form factor fits due to the relative lack of high $Q^{2}$ data. This is also why the $\chi_{\max }^{2}$ cut was applied to the charge form factor instead of the magnetic. These fits are again greatly cleaned up once the $\chi_{\max }^{2}$ cut is applied.

Figure $4.3 \mathrm{~b}$ shows the resulting magnetic form factors of each of the 852 fits of the ${ }^{3} \mathrm{He}$ world data that survive the $\chi_{\max }^{2}=500$ cut plotted together. Immediately it is obvious that the cut has removed most of the nonphysical and bizarre looking results. However, unlike the charge form factor we are no longer in excellent agreement with the fit from [29]. This is to be expected because the new data points from [3] and the point from this analysis add many new high $Q^{2}$ data points to the world data. The magnetic form factor is mostly influenced by these high $Q^{2}$ points unlike the charge form factor which is more influenced by low $Q^{2}$ points. So it is unsurprising that the charge form factor remains the same while the magnetic form factor evolved with the addition of new data. Indeed, the new fits with the new datasets added indicate that the first diffractive minimum for the ${ }^{3} \mathrm{He}$ magnetic form factor is a few femtometers higher in $Q^{2}$ than previously predicted. The consensus of new fits places this minimum at $19-20 \mathrm{fm}^{-2}$ as opposed to the previous fit from [29] which placed the minimum at $18 \mathrm{fm}^{-2}$.

Now that we have functions describing the form factors we can calculate the charge densities as discussed in Section 4.2 . Figure 4.4 shows the charge densities for ${ }^{3} \mathrm{He}$ prior to the $\chi_{\max }^{2}$ cut's application in $4.4 \mathrm{a}$ as well as the charge density for ${ }^{3} \mathrm{He}$ with a $\chi_{\max }^{2}$ cut $=500 \mathrm{in} 4.4 \mathrm{~b}$. Without the cut the charge density shapes are quite variable at small radii and vary widely in magnitude. However, once the nonphysical fits are removed by the $\chi_{\max }^{2}$ cut the only charge densities remaining are extremely consistent in shape and magnitude. Below a radius of about $0.5 \mathrm{fm}$ the charge density comes to a relatively stable plateau. We also note that the charge density tapers off by a radius of $5 \mathrm{fm}$ justifying our guess of $R_{\max }$ in Section 4.3.1.

Using Equation 1.19 we can also calculate the RMS charge radii for each of the 


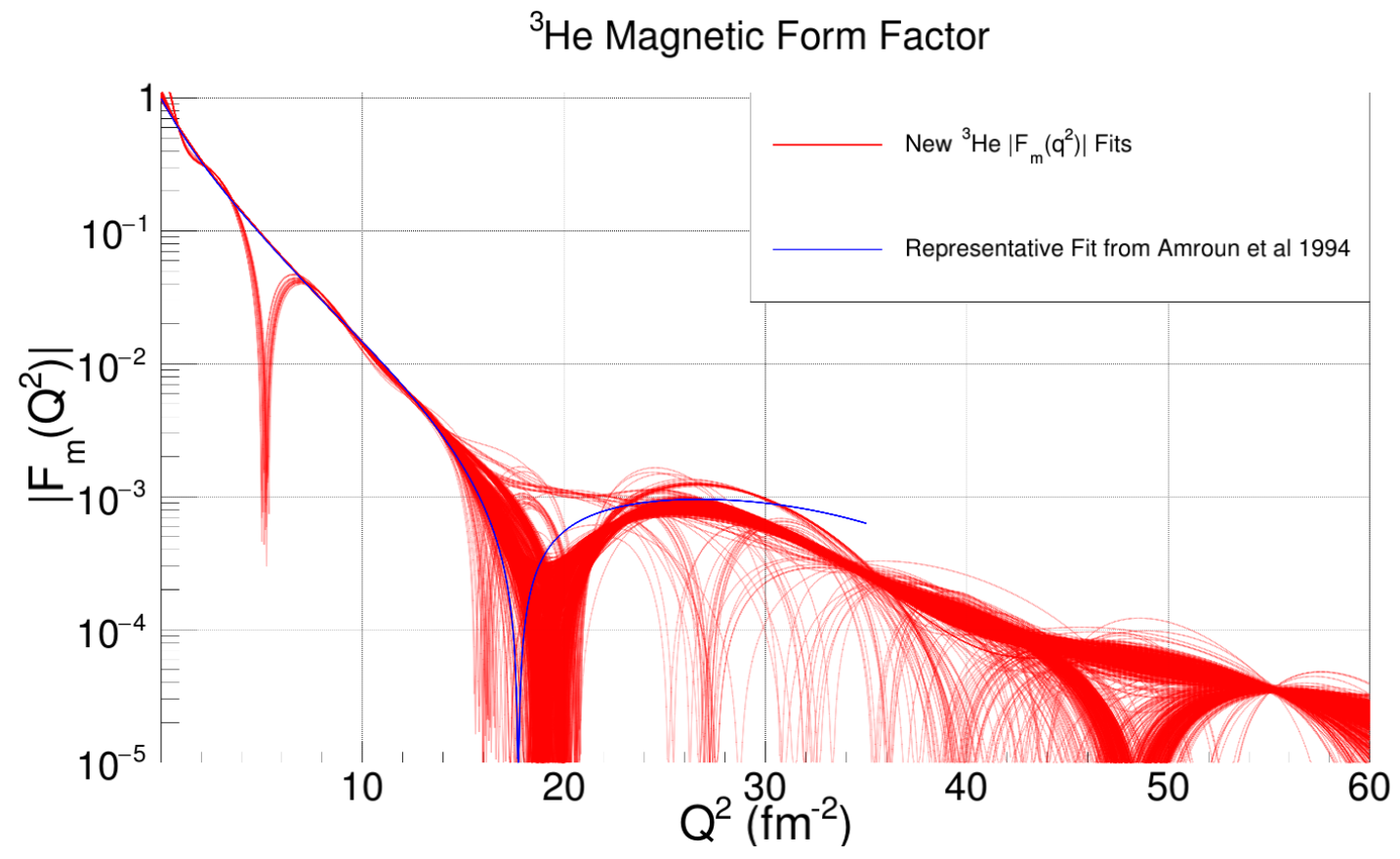

(a) Magnetic Form Factors from $1352{ }^{3} \mathrm{He}$ Fits with no $\chi_{\max }^{2}$ cut. ${ }^{3} \mathrm{He}$ Magnetic Form Factor

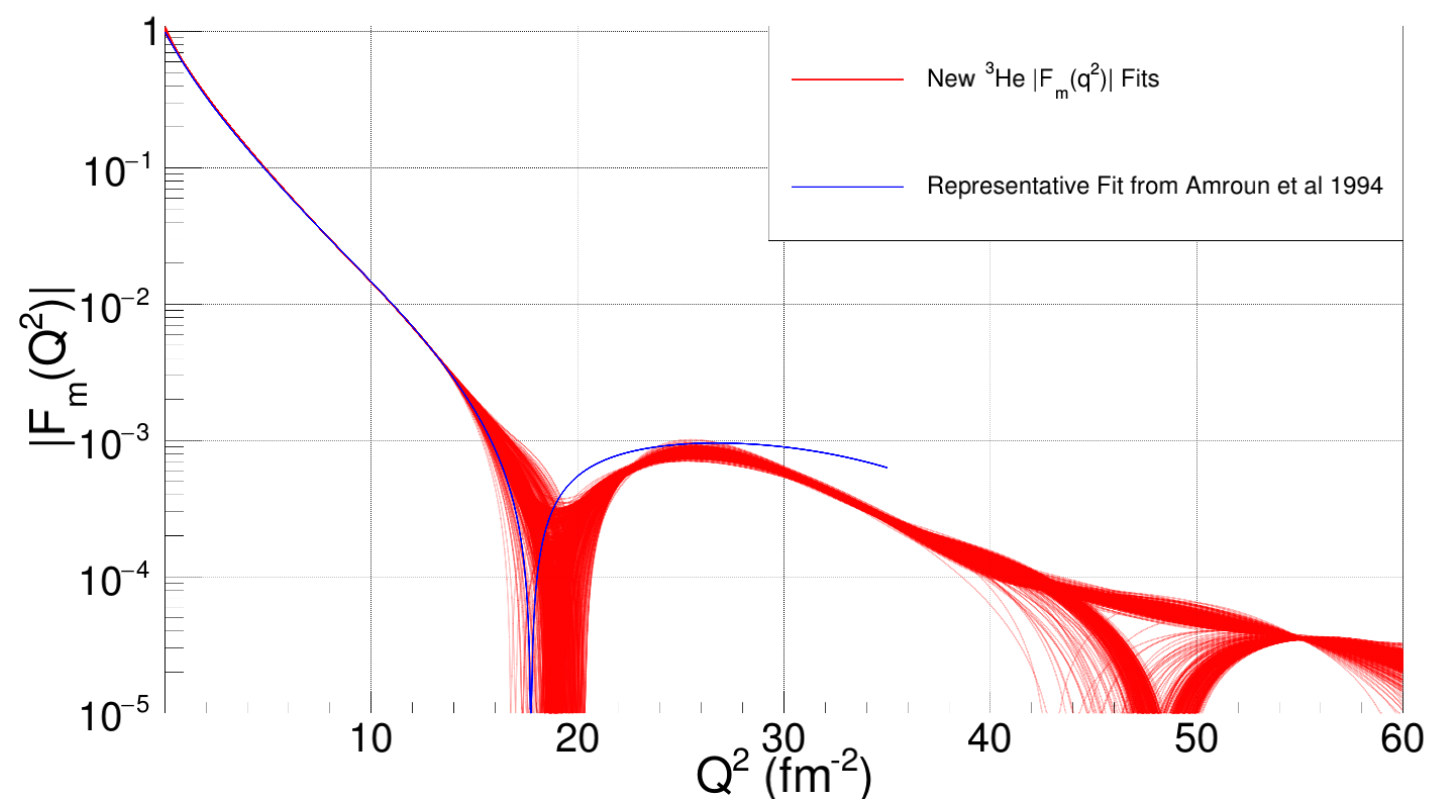

(b) Magnetic Form Factors from $852{ }^{3}$ He Fits surviving a $\chi_{\max }^{2}=500$ cut.

Figure 4.3: ${ }^{3}$ He Magnetic Form Factors. These Magnetic form factors (red lines) were derived from 1352 fits of the ${ }^{3} \mathrm{He}$ world data using pseudorandom initial $R_{i}$ values and the $R_{i}$ optimization procedure. The blue line is the fit from [29]. 


\section{${ }^{3} \mathrm{He}$ Charge Density}

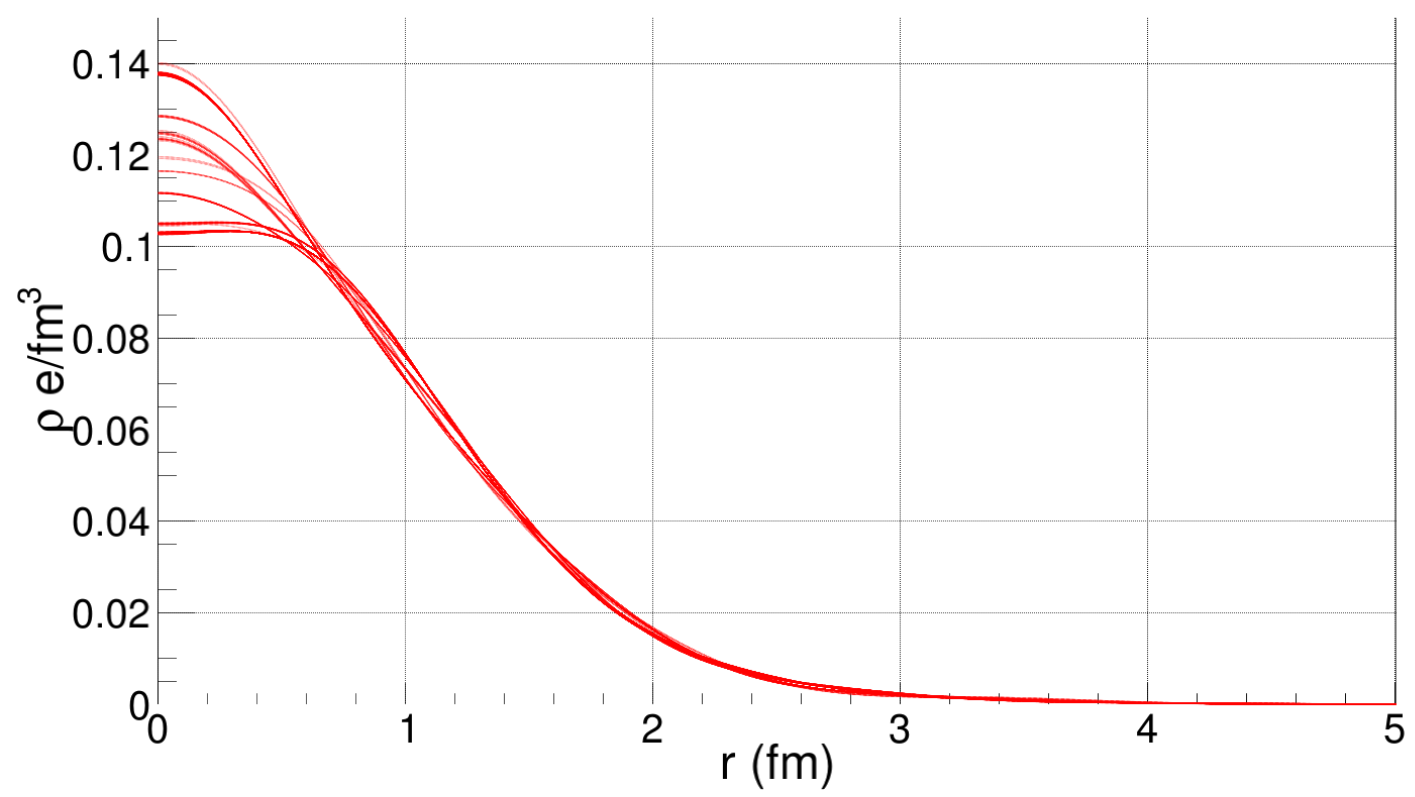

(a) Charge densities of $1352{ }^{3} \mathrm{He}$ fits with no $\chi_{\max }^{2}$ cut.

${ }^{3} \mathrm{He}$ Charge Density

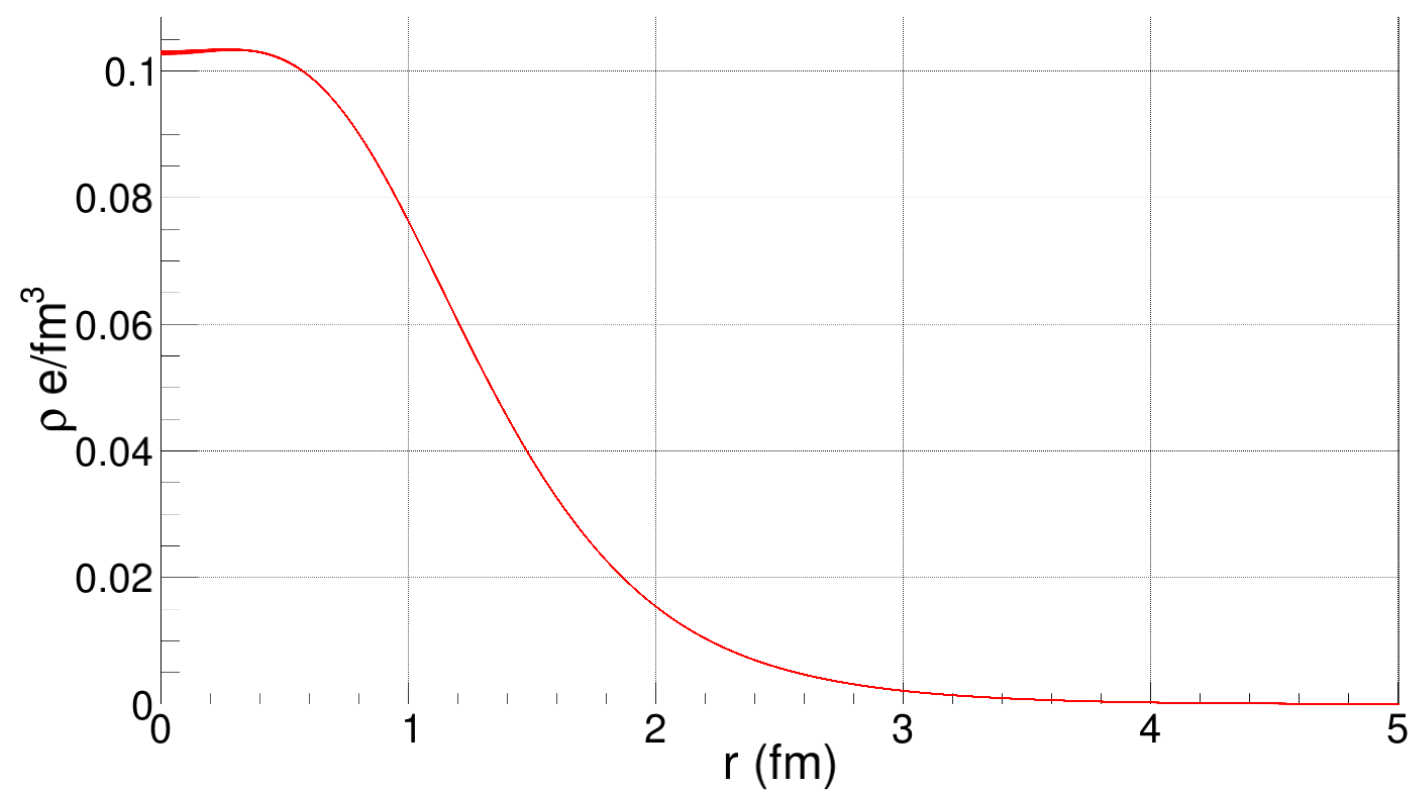

(b) Charge densities of $852{ }^{3} \mathrm{He}$ fits surviving a $\chi_{\max }^{2}=500$ cut.

Figure 4.4: ${ }^{3} \mathbf{H e}$ Charge Densities. The units of the $Y$-axis are the elementary electron charge per cubic fermi.

individual fits. Figure 4.5 shows a plot of the 1352 charge radii resulting from the fits of ${ }^{3} \mathrm{He}$ world data. Figure $4.5 \mathrm{a}$ shows the 1352 fits without a $\chi_{\max }^{2}$ cut, and $4.5 \mathrm{~b}$ 
shows the 852 fits surviving the $\chi_{\max }^{2}=500$ cut. Notice that after the cut is applied the second peak at higher radii disappears indicating that it was nonphysical form factors that were yielding these larger radii. The average of all the RMS charge radii surviving the fit was $1.90 \mathrm{fm}$. A Gaussian fit to these radii finds a mean of $1.90 \mathrm{fm}$ with a standard deviation of $0.00144 \mathrm{fm}$. We see that the radii are well grouped and form a reasonable semblance of a Gaussian distribution. Note that the $R_{i}$ used for each fit were selected in discrete increments of $0.1 \mathrm{fm}$, but if the $R_{i}$ were chosen continuously a better Gaussian for the charge radii distribution might be obtained.

It must be noted that the standard deviation of the charge radii shown here does not represent the full uncertainty on the charge radius. This is because the $\sum Q_{i}$ were not forced to equal one as discussed in Section 4.3.2. By allowing the $\sum Q_{i}$ to float freely this analysis has diverged from the method used by most other charge radii measurements derived from form factors. There is an additional uncertainty on the charge radius in this analysis derived from the uncertainty in the $\sum Q_{i}$. It would have been ideal to quantify this uncertainty by fixing the $\sum Q_{i}$ to different values and studying how this influenced the resultant charge radii. Unfortunately, the code initially written to perform these fits uses Minuit which is not able to fix relations between different free parameters in this manner 49.

It is hoped that a future study of this uncertainty could be made using a more flexible fitting code, but until that analysis is preformed one must keep in mind the additional uncertainty on the charge radii which is not given in this thesis. This additional uncertainty is smaller for ${ }^{3} \mathrm{He}$ than for ${ }^{3} \mathrm{H}$ since the $\sum Q_{i}$ were closer to unity for ${ }^{3} \mathrm{He}$ than ${ }^{3} \mathrm{H}$. This $\sum Q_{i}$ uncertainty is also larger for the magnetic form factors than the charge form factors and is the reason magnetic radii are not calculated in this analysis. For example, the $\sum Q_{i_{c h}}$ for ${ }^{3} \mathrm{He}$ is about 1.008 which quite close to unity so the additional uncertainty on the charge radius for ${ }^{3} \mathrm{He}$ should be relatively small. It would also be informative to refit individual datasets that quote a charge radii that forced the $\sum Q_{i}$ to equal one without this requirement to attempt to quantify any additional uncertainty introduced to their charge radii due to forcing this relation 


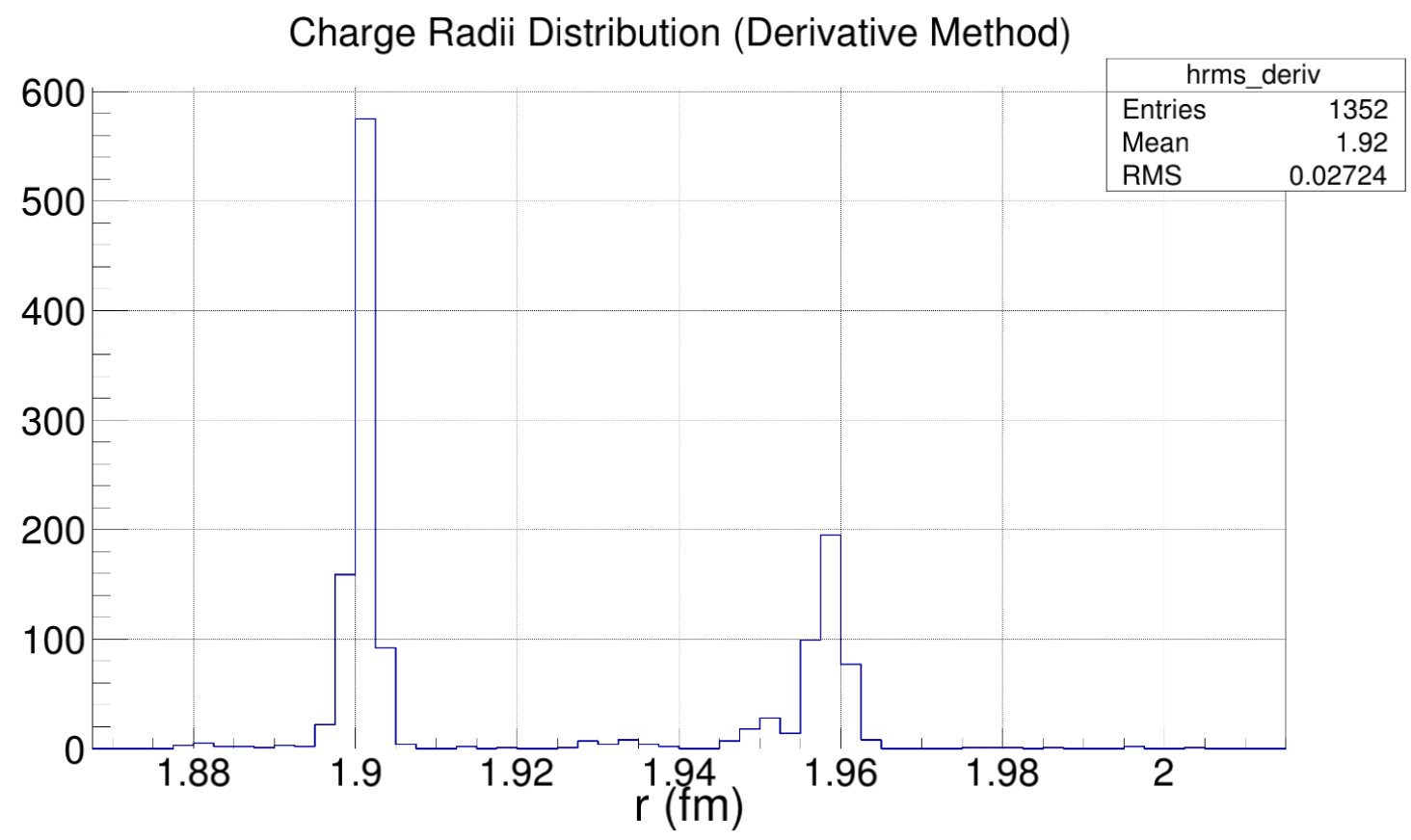

(a) RMS charge radii of $1352{ }^{3}$ He fits with no $\chi_{\max }^{2}$ cut. Charge Radii Distribution (Derivative Method)

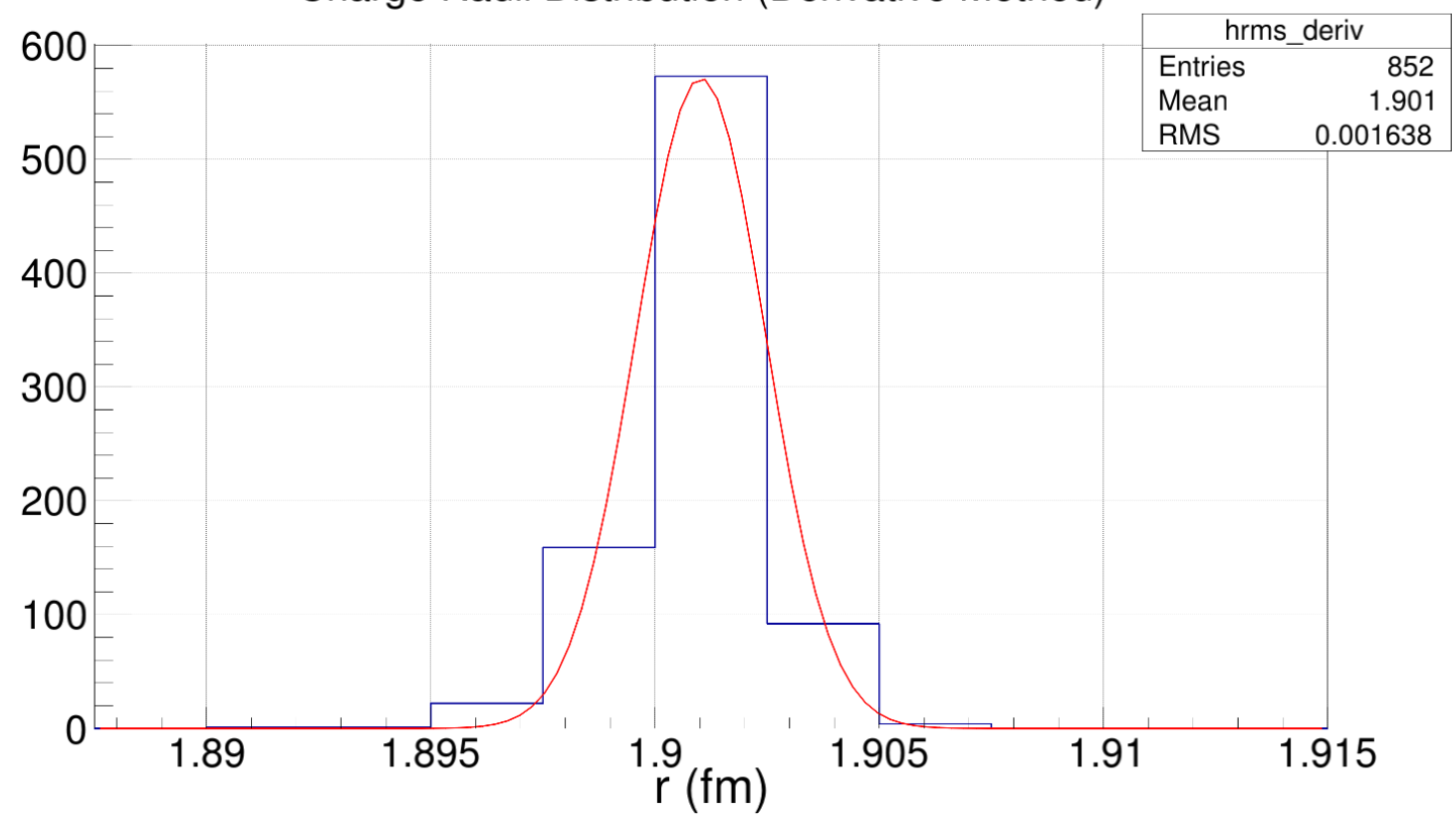

(b) RMS charge radii of $852{ }^{3} \mathrm{He}$ fits surviving a $\chi_{\max }^{2}=500$ cut.

Figure 4.5: ${ }^{3}$ He RMS Charge Radii.

between their 'free' parameters.

The parameters of the ${ }^{3} \mathrm{He}$ fits can be plotted as well for further information. Figure 4.6 shows the $Q_{i}$ fit parameters discussed in 4.2 with Figure 4.6 a showing the $Q_{i_{c h}}$ 
parameters and Figure $4.6 \mathrm{~b}$ showing the $Q_{i_{m}}$ parameters. Each of the 12 parameters is shown up to a maximum value of 0.5 . The structure of the parameter plots did not vary significantly after the $\chi_{\max }^{2}$ cut was applied so only the plots with the cut are shown. One important thing to notice is that the $Q_{i}$ values are larger for the inner Gaussians placed at smaller radii. The inner Gaussians have far more influence over the fit than the outer ones which makes since as most of the structure of the charge density is located at smaller radii as well. A small change in $Q_{i_{1-3}}$ can have drastic effects on the form factors, but small changes to the higher $Q_{i}$ have almost no influence on the form factors.

Also note that the inner $Q_{i}$ parameters have a much wider spread in values than the outer $Q_{i}$. This is because the fits are extremely sensitive to the final combination of $R_{i}$ selected which flow from the pseudorandom $R_{i}$ initially generated for each fit. This means that the $Q_{i}$ values vary widely from fit to fit as seen in the large spread of the inner parameters. As such, attributing an uncertainty to each parameter is fairly meaningless since each set of parameters is representative only of the specific 'model' created by the $R_{i}$ of that single fit. It would be satisfying if the average value of each $Q_{i}$ and $R_{i}$ could be found and then used to plot an average set of form factors. However, due to the strong correlation between the $Q_{i}$ and $R_{i}$ when this was attempted the fit was very poor and nonphysical in nature.

Plotted in Figure 4.7 are the $R_{i}$ values $4.7 \mathrm{a}$ and the separation between consecutive $R_{i}$ values $4.7 \mathrm{~b}$ of the fits of the ${ }^{3} \mathrm{He}$ world data in $\mathrm{fm}$. Little difference was made after the $\chi_{\max }^{2}$ cut was applied so only the final 852 fits surviving the cut were plotted. The individual $R_{i}$ are seen to be fairly well grouped around the same values. This is especially true for the lower radii which have more influence over the fits than the large radii due to their larger $Q_{i}$ values. The consistent positioning of the radii after the optimization procedure described in 4.3 .1 demonstrates that the pseudorandom $R_{i}$ generated tend to converge to consistent values and not wander randomly.

We also see that our prior that $R_{\max }$ will be $\approx 5 \mathrm{fm}$ agrees with the largest $R_{i}$ after the fitting procedure. Finally, we notice that the distance between consecutive $R_{i}$ 

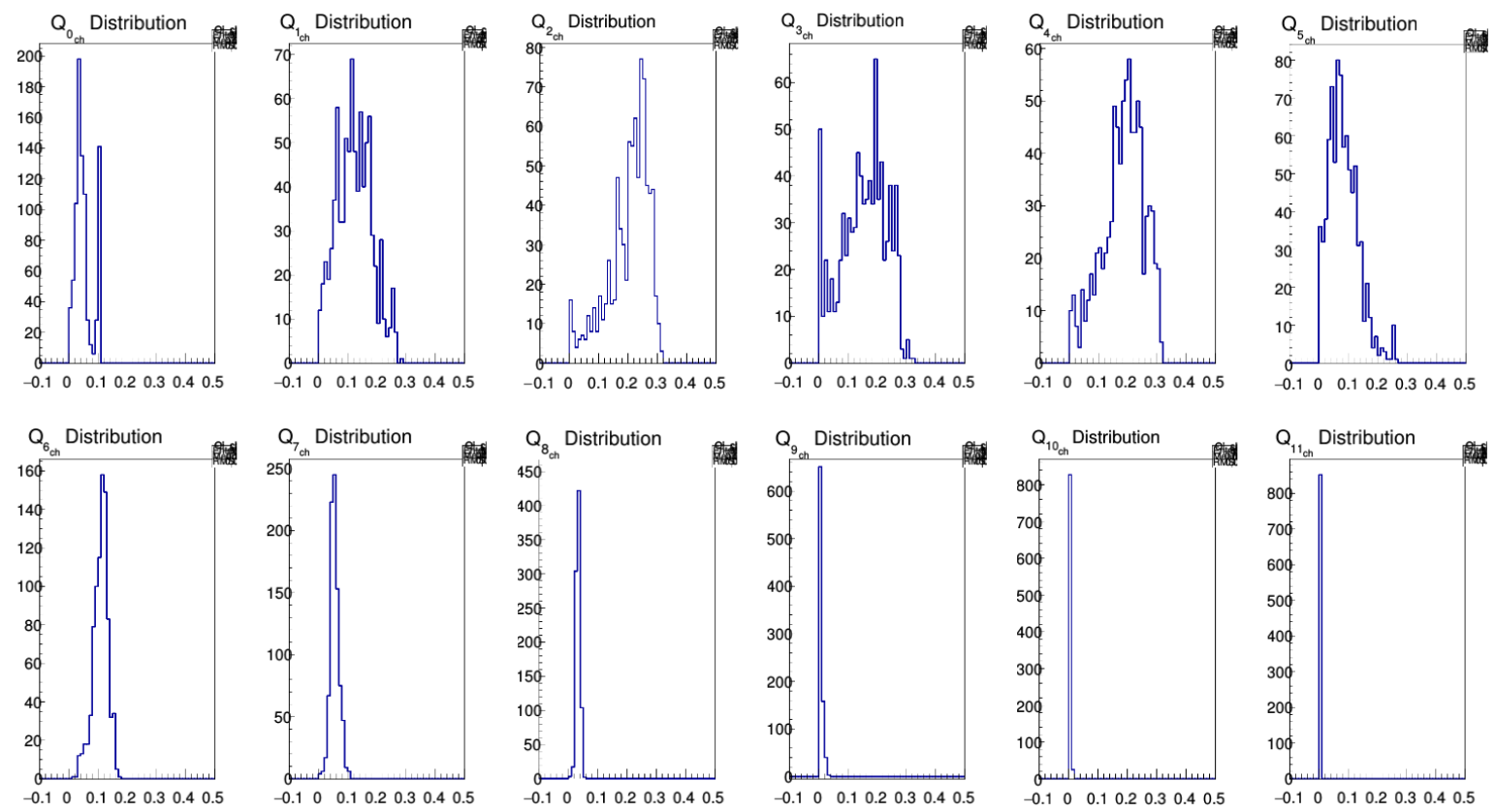

(a) $Q_{i_{c h}}$ parameters for ${ }^{3} \mathrm{He}$ with $\chi_{\max }^{2}=500$.
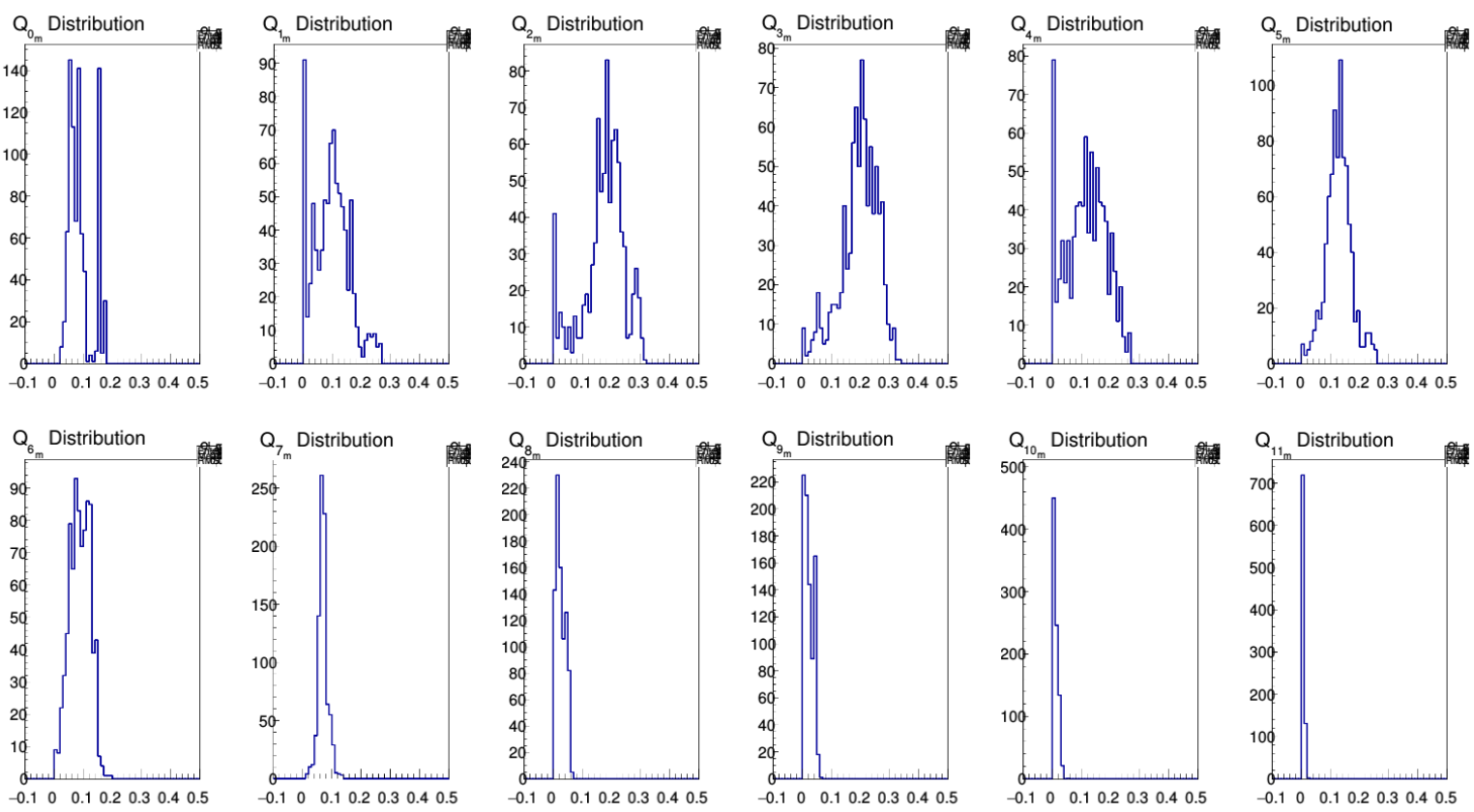

(b) $Q_{i_{m}}$ parameters for ${ }^{3} \mathrm{He}$ with $\chi_{\max }^{2}=500$.

Figure 4.6: ${ }^{3} \mathbf{H e} \boldsymbol{Q}_{\boldsymbol{i}}$ Fit Parameters. The $X$-axis of these plots is the $Q_{i}$ value in the range of -0.1 to 0.5 . The $Y$-axis is the number of counts. The plots run from $Q_{0}$ up to $Q_{11}$. Notice that the distributions are each roughly centered about a specific value showing that the SOG fits are consistently finding similar $Q_{i}$ values. Also notice that the smaller radii parameters generally have larger values. 
values does fluctuate somewhat, but the separations still tend to converge to a central value, particularly at the lower radii. We had previously guessed that the separation between consecutive $R_{i}$ would roughly double for $R_{i}>R_{\max } / 2$ which is also consistent with these results.

The set of new world data fits for ${ }^{3} \mathrm{He}$ have essentially created an uncertainty band for the form factors by spanning the set of $R_{i}$ models. To make these new fits applicable a specific parametrization of the form factors is required. To select this representative fit for ${ }^{3} \mathrm{He}$ a single fit at the middle of the distribution of all new fits, with physical characteristics and reasonable values for the metrics listed in Table 4.3 , was selected. Table 4.6 and Table 4.7 show the parameters for the representative fit of the ${ }^{3} \mathrm{He}$ world data along with the metrics used to test the 'goodness' of the fit respectively.

\begin{tabular}{|ccll|}
\hline $\begin{array}{c}\text { Parameter } \\
\text { Number }\end{array}$ & $\boldsymbol{R}_{\boldsymbol{i}}(\mathbf{f m})$ & $\boldsymbol{Q}_{\boldsymbol{i}_{\boldsymbol{c h}}}$ & $\boldsymbol{Q}_{\boldsymbol{i}_{\boldsymbol{m}}}$ \\
\hline 1 & 0.3 & 0.0996392 & 0.159649 \\
2 & 0.7 & 0.214304 & 0.0316168 \\
3 & 0.9 & 0.0199385 & 0.277843 \\
4 & 1.1 & 0.195676 & 0.0364955 \\
5 & 1.5 & 0.0785533 & 0.0329718 \\
6 & 1.6 & 0.167223 & 0.233469 \\
7 & 2.2 & 0.126926 & 0.117059 \\
8 & 2.7 & 0.0549379 & 0.0581085 \\
9 & 3.3 & 0.0401401 & 0.0485212 \\
10 & 4.2 & 0.0100803 & $1.77602 \times 10^{-12}$ \\
11 & 4.3 & 0.0007217 & 0.0240927 \\
12 & 4.8 & $4.98962 \times 10^{-12}$ & $8.94934 \times 10^{-12}$ \\
\hline
\end{tabular}

Table 4.6: Parameters for ${ }^{3}$ He World Data Representative Fit. These fit parameters give the analytical form factors when plugged into Equation 4.4 .

Figure 4.8 shows plots of the representative charge and magnetic form factors for the new ${ }^{3} \mathrm{He}$ world data fits. The charge form factor error band is very well determined until $Q^{2} \approx 55 \mathrm{fm}^{-2}$ where it begins to expand. (Note that the error bands used for ${ }^{3} \mathrm{He}$ and ${ }^{3} \mathrm{H}$ in this thesis were created and/or digitized with the incredibly useful free tool [54.) The magnetic form factor is very well determined below $Q^{2} \approx 12 \mathrm{fm}^{-2}$. 

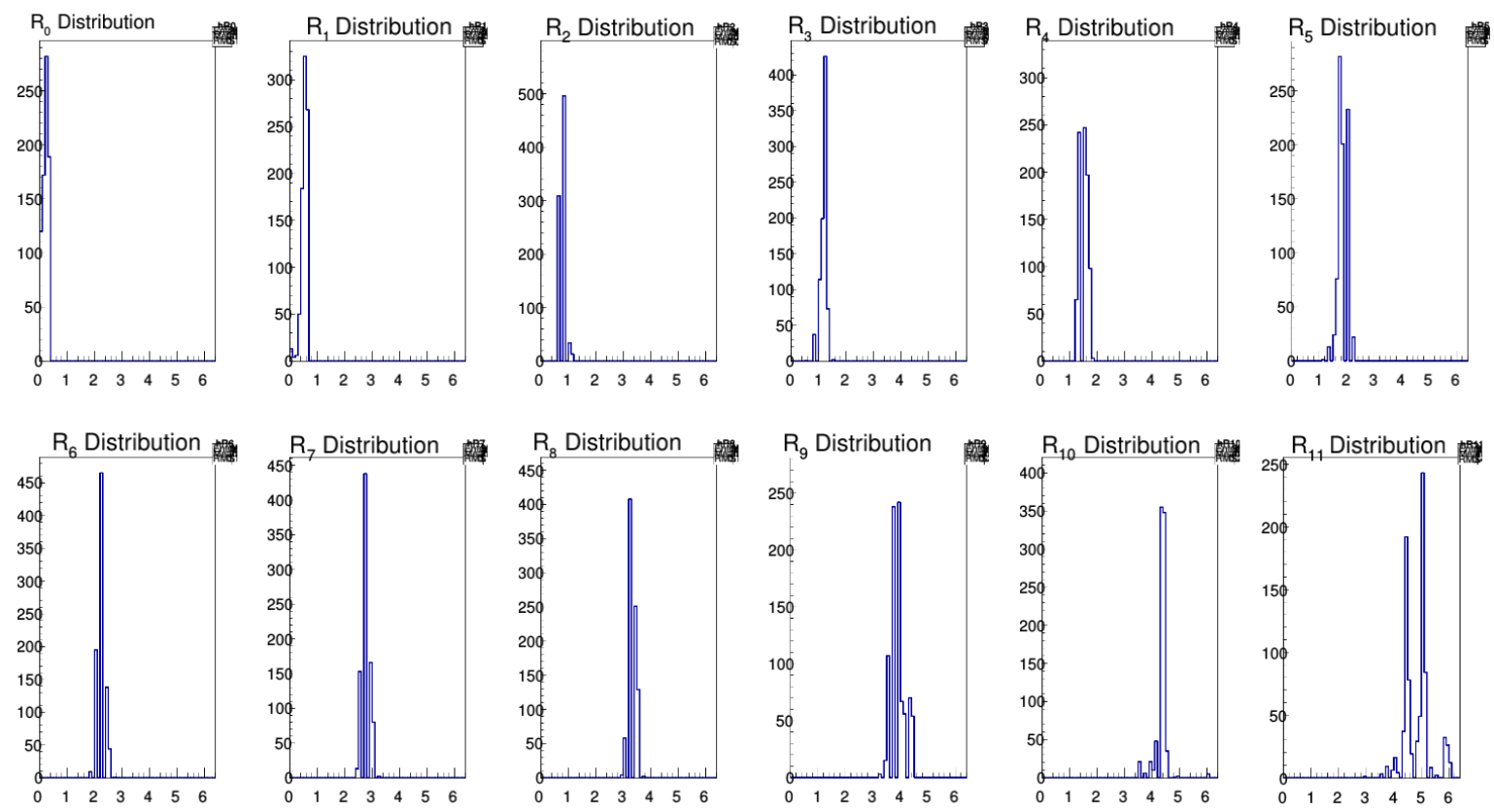

(a) $R_{i}$ values $(\mathrm{fm})$ of fits of ${ }^{3} \mathrm{He}$ world data $\chi_{\max }^{2}=500$.
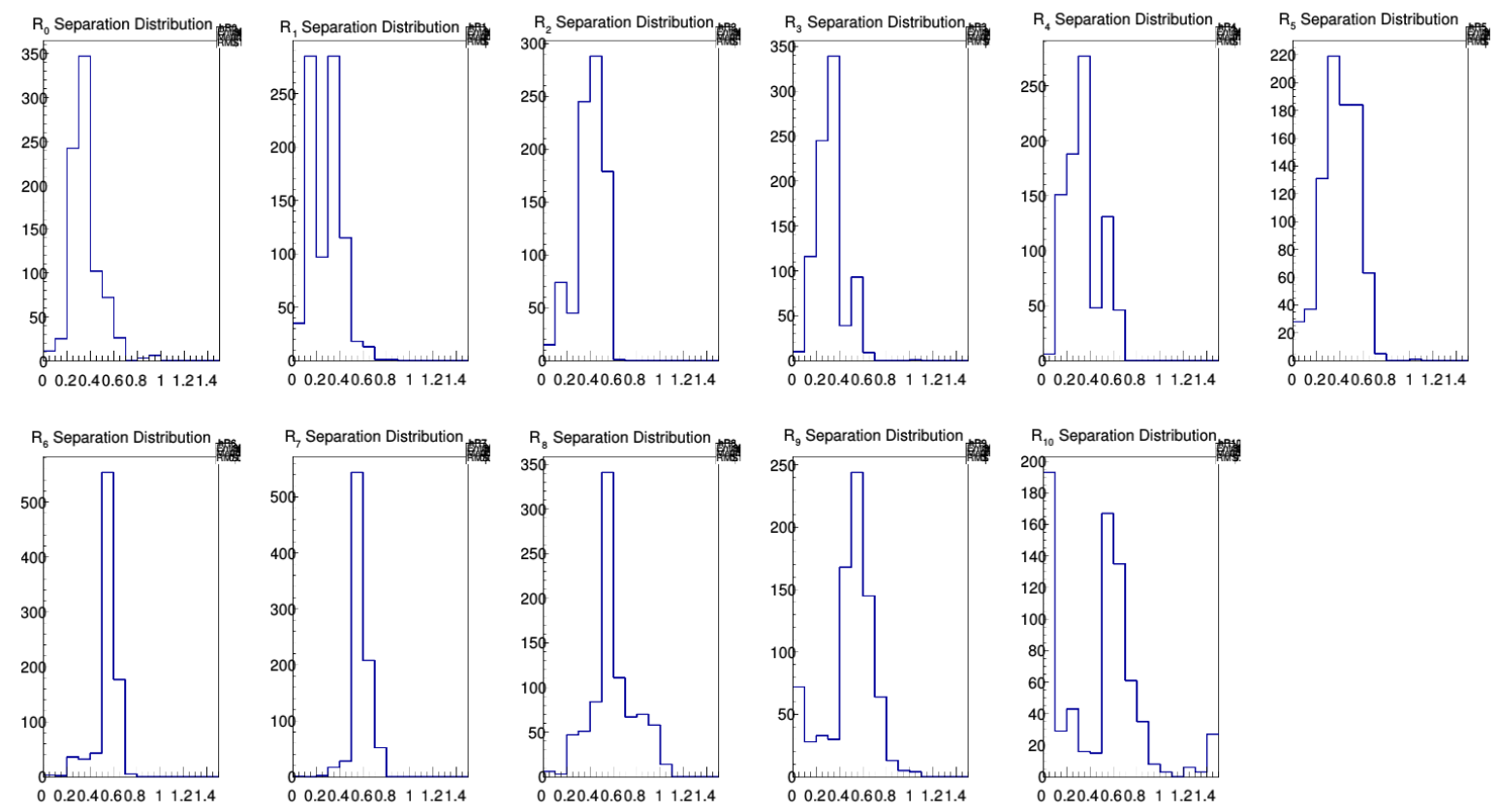

(b) Separation of consecutive $R_{i}$ values ( $\mathrm{fm}$ ) of fits of ${ }^{3} \mathrm{He}$ world data $\chi_{\max }^{2}=500$.

Figure 4.7: ${ }^{3}$ He $\boldsymbol{R}_{\boldsymbol{i}}$ Values and Separations. The top plot shows the values for $R_{i}$ from $R_{0}$ up to $R_{11}$, and the bottom plot shows the distance separating each consecutive $R_{i}$, where the $X$-axes are in $\mathrm{fm}$. Notice that each plot is roughly centered about a specific value showing that the SOG fits are consistently finding similar $R_{i}$ values and separations. Also notice that the distributions widen at larger radii. 


\begin{tabular}{|ccccccc|}
\hline $\boldsymbol{N}_{\text {Gaus }}$ & Avg. $\chi^{\mathbf{2}}$ & $\chi_{\boldsymbol{r}}^{\mathbf{2}}$ & BIC & AIC & $\sum \boldsymbol{Q}_{\boldsymbol{i}_{\text {ch }}}$ & $\sum \boldsymbol{Q}_{\boldsymbol{i}_{\boldsymbol{m}}}$ \\
\hline 12 & 436.1 & 1.864 & 201.6 & 158.9 & 1.008 & 1.020 \\
\hline
\end{tabular}

Table 4.7: Metrics for ${ }^{3} \mathbf{H e}$ World Data Representative Fit. $N_{\text {Gaus }}$ is the number of Gaussians used in the SOG fits, Avg. $\chi^{2}$ is the average $\chi^{2}$ value for the fits, $\chi_{r}^{2}$ is the average reduced $\chi^{2}$ value for the fits, BIC is the average Bayesian information criterion value of the fits, AIC is the average Akaike information criterion value of the fits, $\sum Q_{i_{c h}}$ is the average sum of the $Q_{i_{c h}}$ fit parameters, and $\sum Q_{i_{m}}$ is the average sum of the $Q_{i_{m}}$ fit parameters.

As $F_{m}$ approaches its first minimum the error band expands considerably producing a range of about $4 \mathrm{fm}^{-2}$ in $Q^{2}$ where the diffractive minimum is located. The error band then tightens again between 22 and $36 \mathrm{fm}^{-2}$ with the addition of the new ${ }^{3} \mathrm{He}$ data from JLab and this analysis. The error band then expands again as a possible second diffractive minimum is approached due to leaving the $Q^{2}$ range in which robust experimental data exists.

The representative fits for the ${ }^{3} \mathrm{He}$ form factors can also be plotted along with the world data and the previous fit from [29] as shown in Figure 4.9. Note that the Amroun fits are deemed valid only up to $35 \mathrm{fm}^{-2}$ but were extended for the sake of comparison. The Cavedon data [4] shown in Figure 4.8b is also in the Amroun paper. The form factor points shown are derived from Rosenbluth separations performed by the experiments used for this analysis' global fits. There are fewer discreet form factor points plotted than there were cross section data points fitted since the experiments did not perform Rosenbluth separations for each $Q^{2}$ value due to the time this would take. Some papers, like Amroun 1994, did not publish their form factor data point values explicitly, but instead only plotted these points so they are not shown. Notice that the world data for $F_{c h}$ agrees quite well with both the new and old fits. The only noticeable change is at $Q^{2} \approx 55 \mathrm{fm}^{-2}$ the new point from Camsonne 2016 pulled the form factor down slightly. $F_{m}$ has changed more with the addition of the new data from Nakagawa 2001, Camsonne 2016, and this analysis' data point. The Nakagawa and Camsonne data have pulled the first difractive minimum higher in $Q^{2}$, and have decreased its magnitude fairly significantly after the first minimum. 


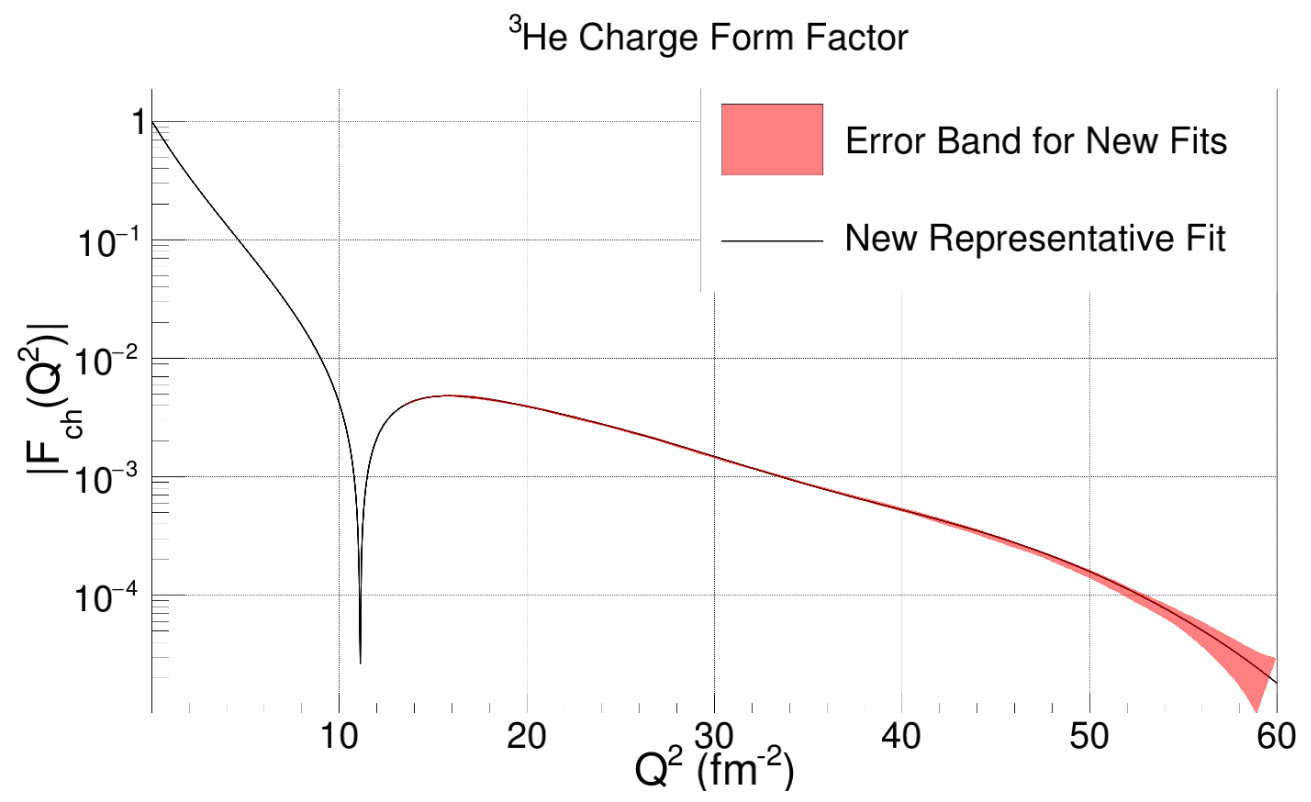

(a) ${ }^{3} \mathrm{He}$ Charge Form Factor for the New Representative Fit of World Data.

${ }^{3} \mathrm{He}$ Magnetic Form Factor

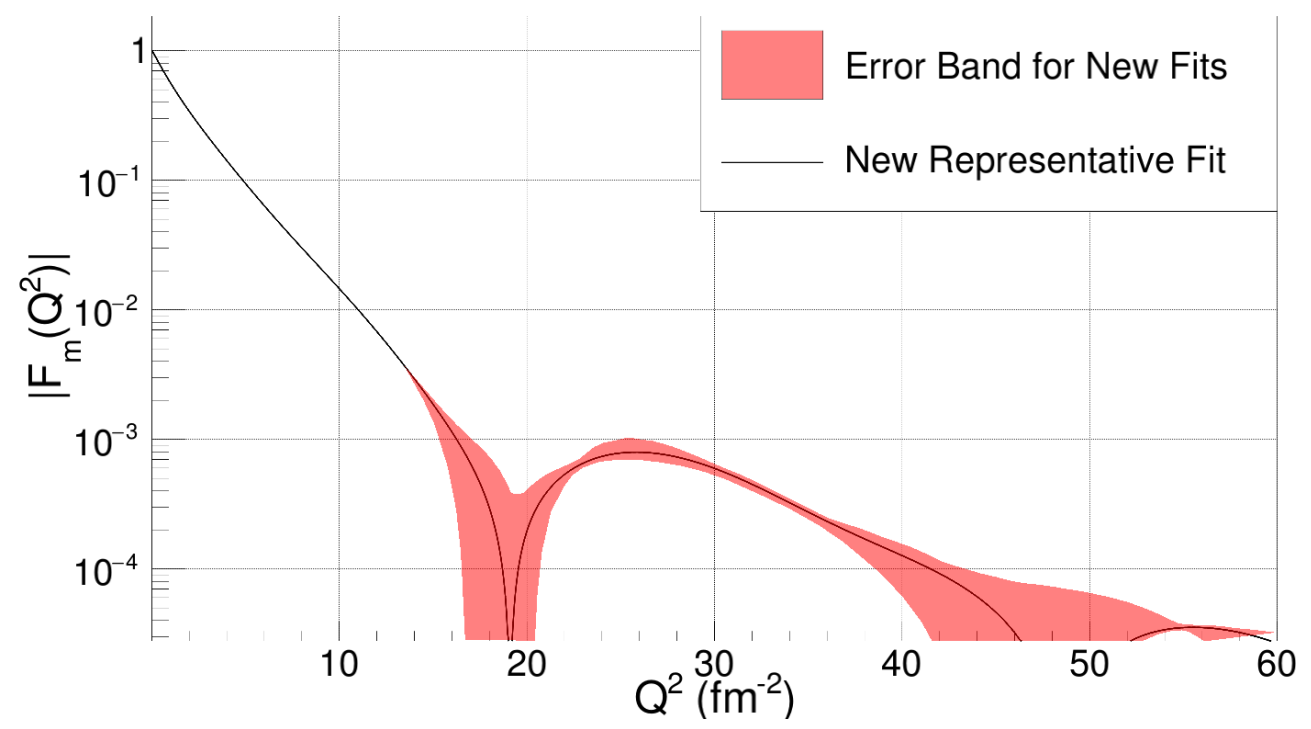

(b) ${ }^{3}$ He Magnetic Form Factor for the New Representative Fit of World Data.

Figure 4.8: ${ }^{3}$ He Form Factors for the New Representative Fit of World Data. The selected representative fit is shown in black and its error band is shaded in red.

Our understanding of the form factors in different kinematic ranges depends on the ${ }^{3} \mathrm{He}$ cross section world data distribution seen in Figure 4.10 . Looking at the distribution of the world data it becomes clear that most of the data exists below $Q^{2}$ $\approx 12 \mathrm{fm}^{-2}$, and above that value it becomes quite sparse. This analysis has previously 


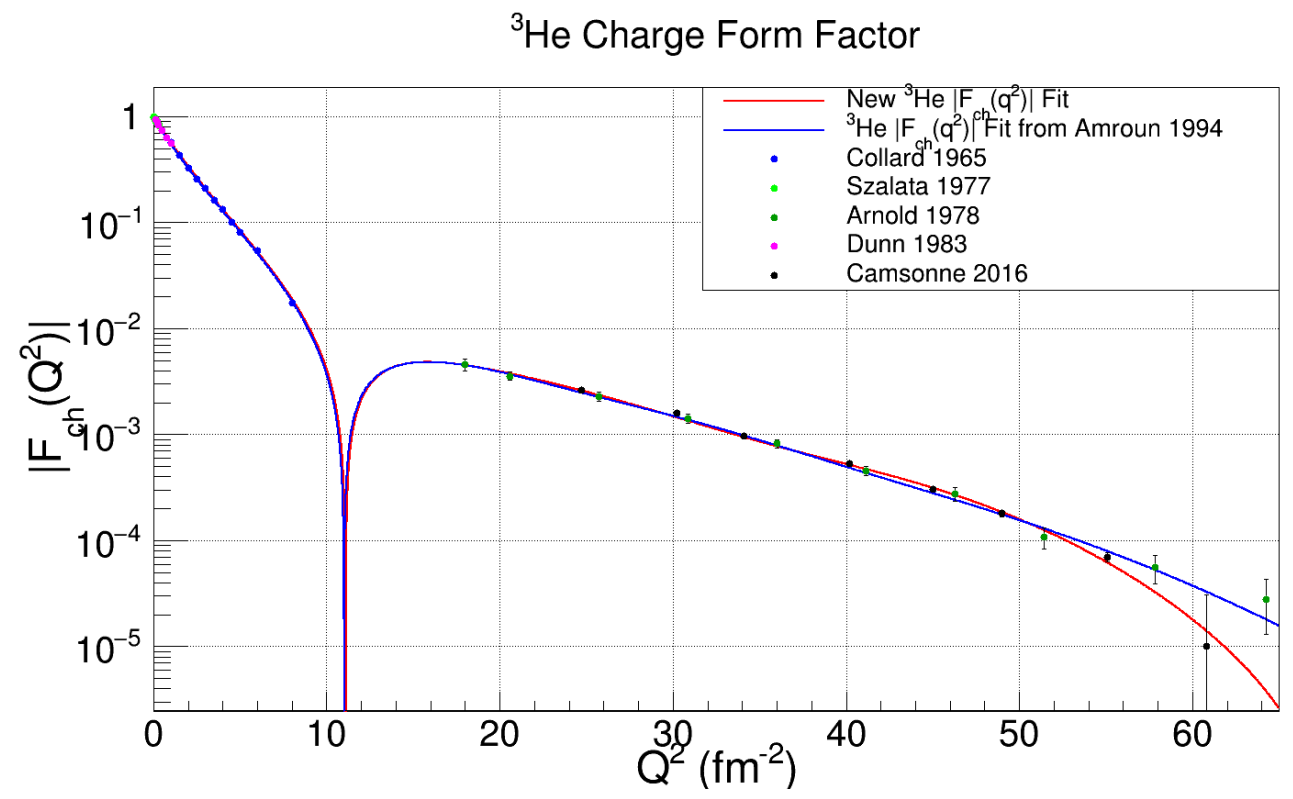

(a) ${ }^{3}$ He Representative Charge Form Factor with World Data and Amroun Fit ${ }^{3} \mathrm{He}$ Magnetic Form Factor

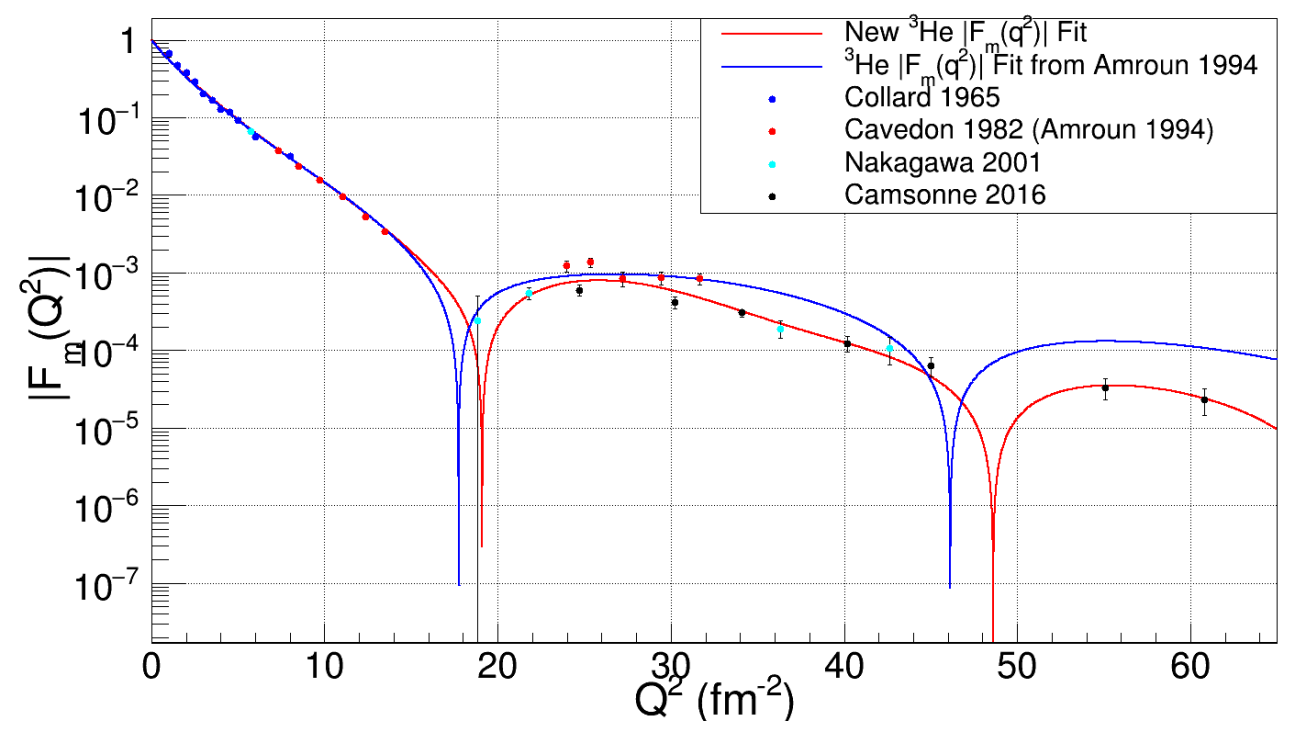

(b) ${ }^{3}$ He Representative Magnetic Form Factor with World Data and Amroun Fit.

Figure 4.9: ${ }^{3}$ He Representative Form Factors with World Data and Amroun Fit. The red lines are the new representative fits. The blue lines are the previous Amroun fits. Note that the Amroun fits are deemed valid only up to $35 \mathrm{fm}^{-2}$ but were extended for the sake of comparison. The points represent published form factor measurements from the datasets used for this analysis' global fits. These points were extracted via standard Rosenbluth separations. 
discussed the need for more high $Q^{2}$ data, and Figure 4.10 reinforces how the dearth of this data is restricting our understanding of the ${ }^{3} \mathrm{He}$ form factors.

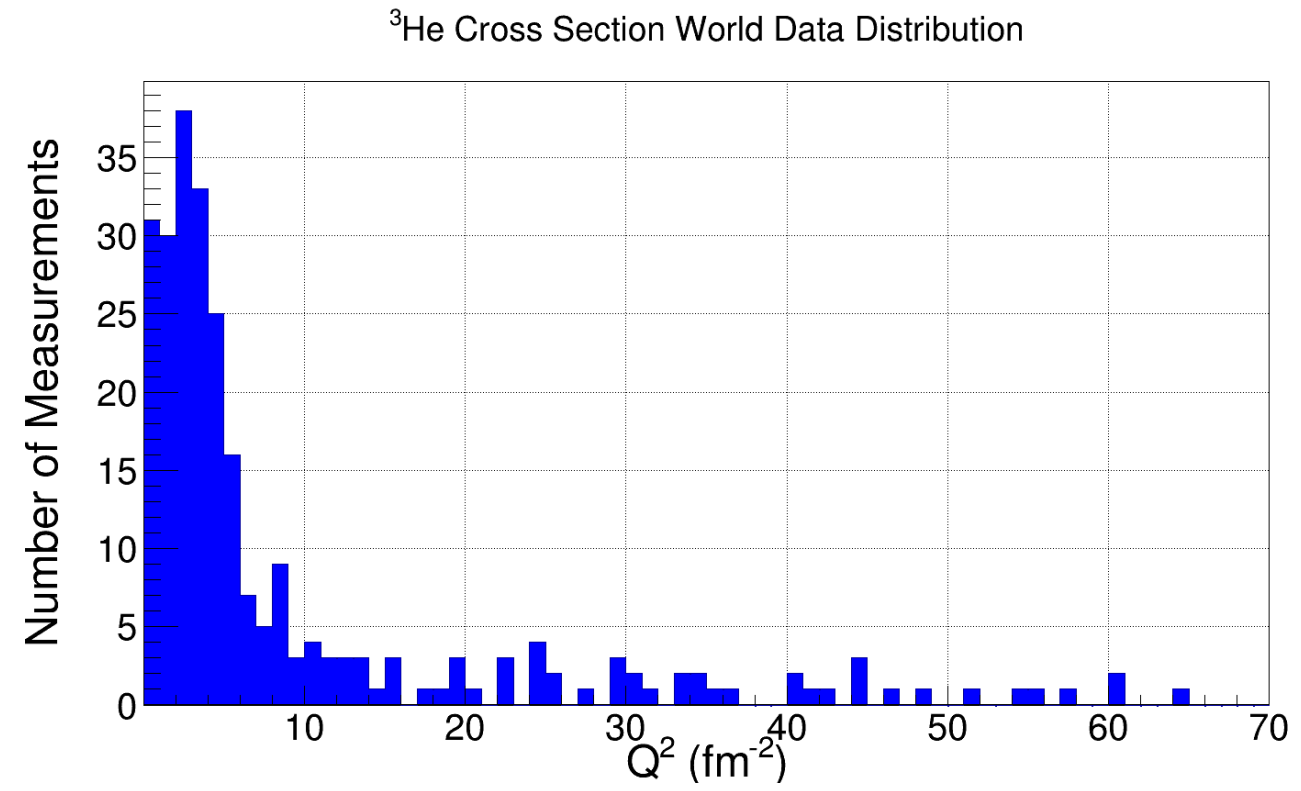

Figure 4.10: ${ }^{3}$ He Cross Section World Data Distribution. This plot shows the ${ }^{3} \mathrm{He}$ cross section world data distribution in $Q^{2}$ used in this analysis.

This representative fit has a total $\chi^{2}$ of 436.1 and a reduced $\chi^{2}$ of 1.864 for 259 ${ }^{3} \mathrm{He}$ elastic cross sections. Recall that this analysis did not float the normalization of the different datasets leading to a slightly larger $\chi^{2}$. When examining how well the representative fit is describing the data it can be helpful to look at the $\chi^{2}$ values for each data individual point in each dataset as shown in Figure 4.11. This plot indicates that much of the total $\chi^{2}$ comes from the older datasets found in Collard and Szalata. The point from this analysis (orange) has a $\chi^{2}$ of $\approx 4$.

It can also be illuminating to look at the residual of the fit for each data point in each dataset as shown in Figure 4.12, Note that since the plot encompasses many orders of magnitude the residual is plotted as Equation 4.13, where $\sigma_{\exp }$ is the experimental cross section measured and $\sigma_{f i t}$ is the cross section predicted by the fit at those same kinematics. Firstly, there are two heavy outliers from Arnold 43], but this is not very surprising since these points were taken at very high $Q^{2}$. Zooming in on the remaining 


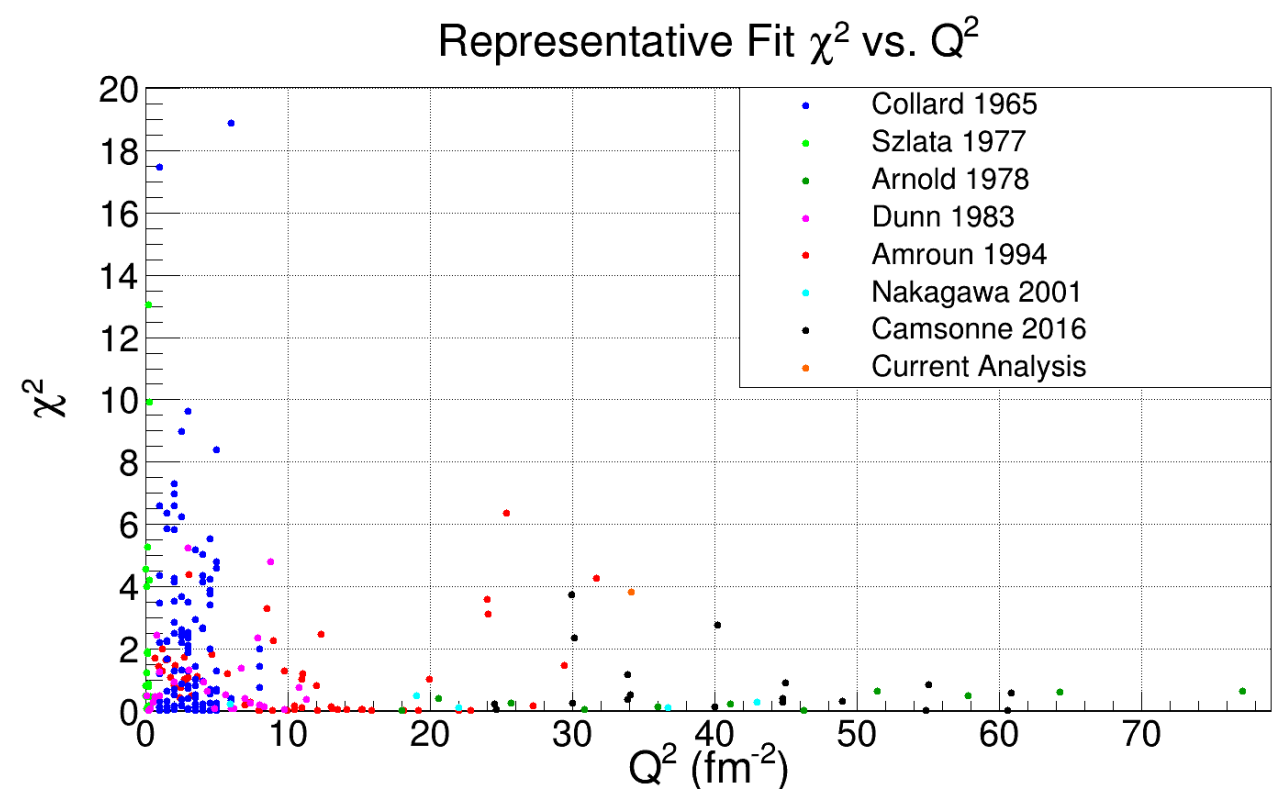

Figure 4.11: ${ }^{3}$ He Representative Fit $\chi^{2}$ vs. $Q^{2}$.

points they mostly look to be normally distributed about zero, although the points from Collard may lie a little high as do some of the high $Q^{2}$ points from Amroun. This indicates that floating the normalizations of the different datasets would not have improved the overall $\chi^{2}$ significantly. We also find that the point from this analysis lies below the representative fit.

$$
\text { Residual }=\frac{\sigma_{e x p}-\sigma_{f i t}}{\sigma_{f i t}}
$$

The representative fits for the charge and magnetic form factor can be combined to yield the full elastic ${ }^{3} \mathrm{He}$ cross section using Equation 4.5 . Figure 4.13 shows the elastic ${ }^{3} \mathrm{He}$ cross section using the kinematics of experiment E08-014 along with this analysis' new data point. The new cross section fit is fairly similar to the previous fit from 29. The new high $Q^{2}$ data, including the point from this analysis, have brought the cross section down slightly in the region of $Q^{2}=30-40 \mathrm{fm}^{-2}$. The new cross section also falls off more quickly above $Q^{2}=50 \mathrm{fm}^{-2}$ than the previous fit fell off. The majority of this movement is attributable to the changes in the magnetic form factor fit. The 
Residual of Representative Fit vs. $Q^{2}$

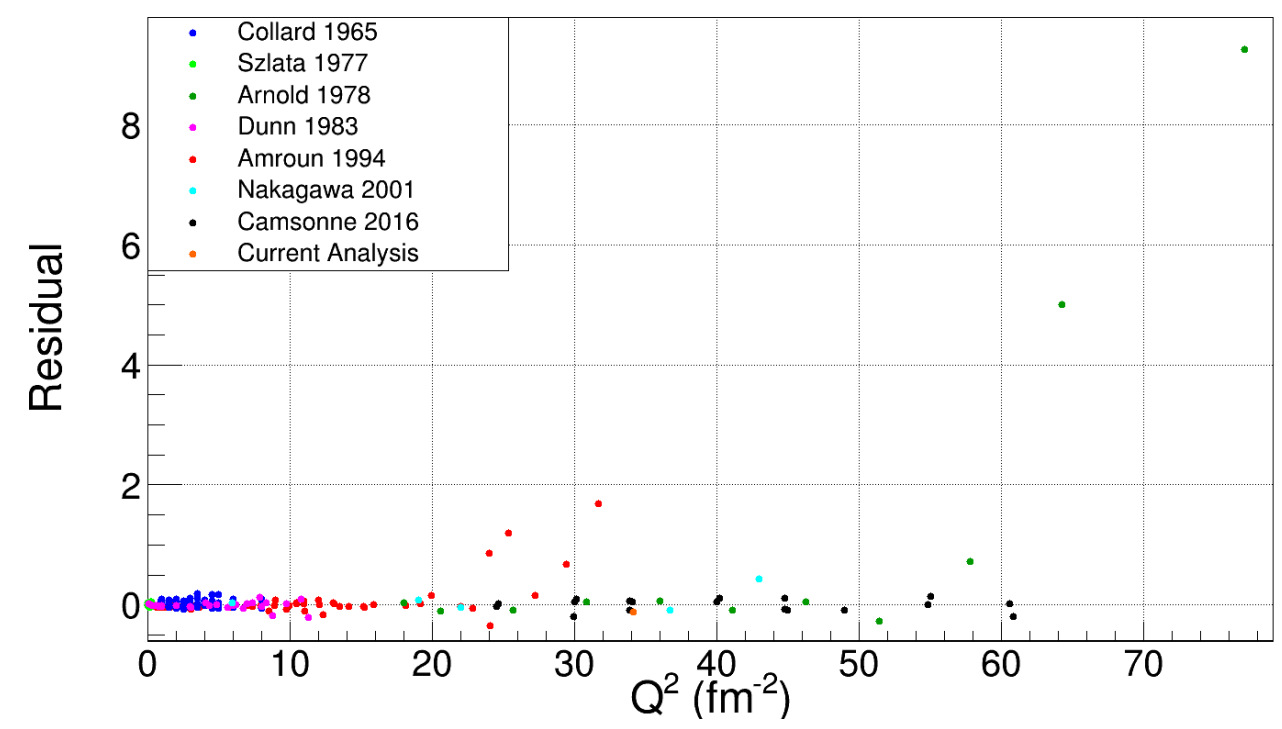

(a) No Zoom.

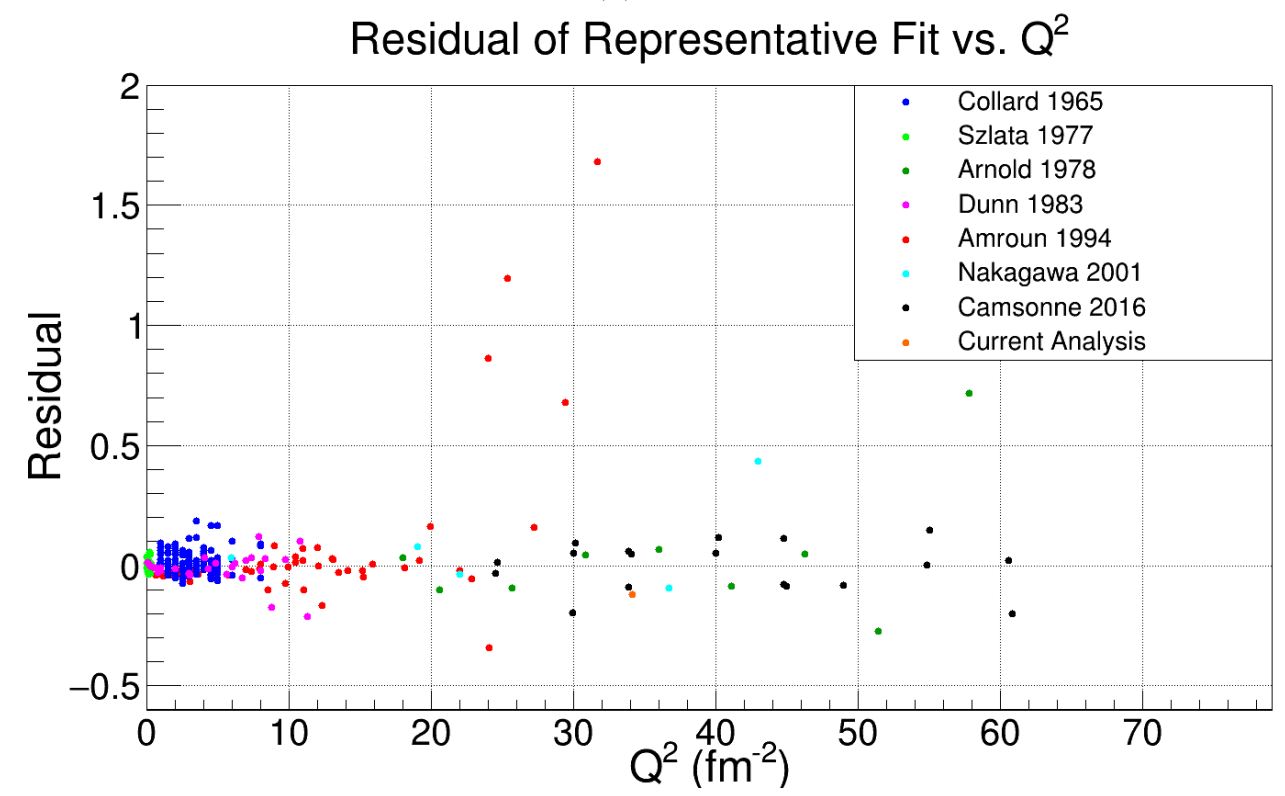

(b) Zoomed In.

Figure 4.12: ${ }^{3}$ He Representative Fit Residual vs. $\boldsymbol{Q}^{\mathbf{2}}$. The upper plot shows a view of each data point's residual, and the lower plot shows a zoomed in version without the two high residual points for clarity.

new cross section measurement from this analysis can be seen better in the zoomed in plot (Figure 4.13b). In this plot we see that the new data point is closer to this analysis' cross section fit. The uncertainty on the new data point nearly overlaps with 
the new cross section fit and is certainly close enough to be consistent with the fit.

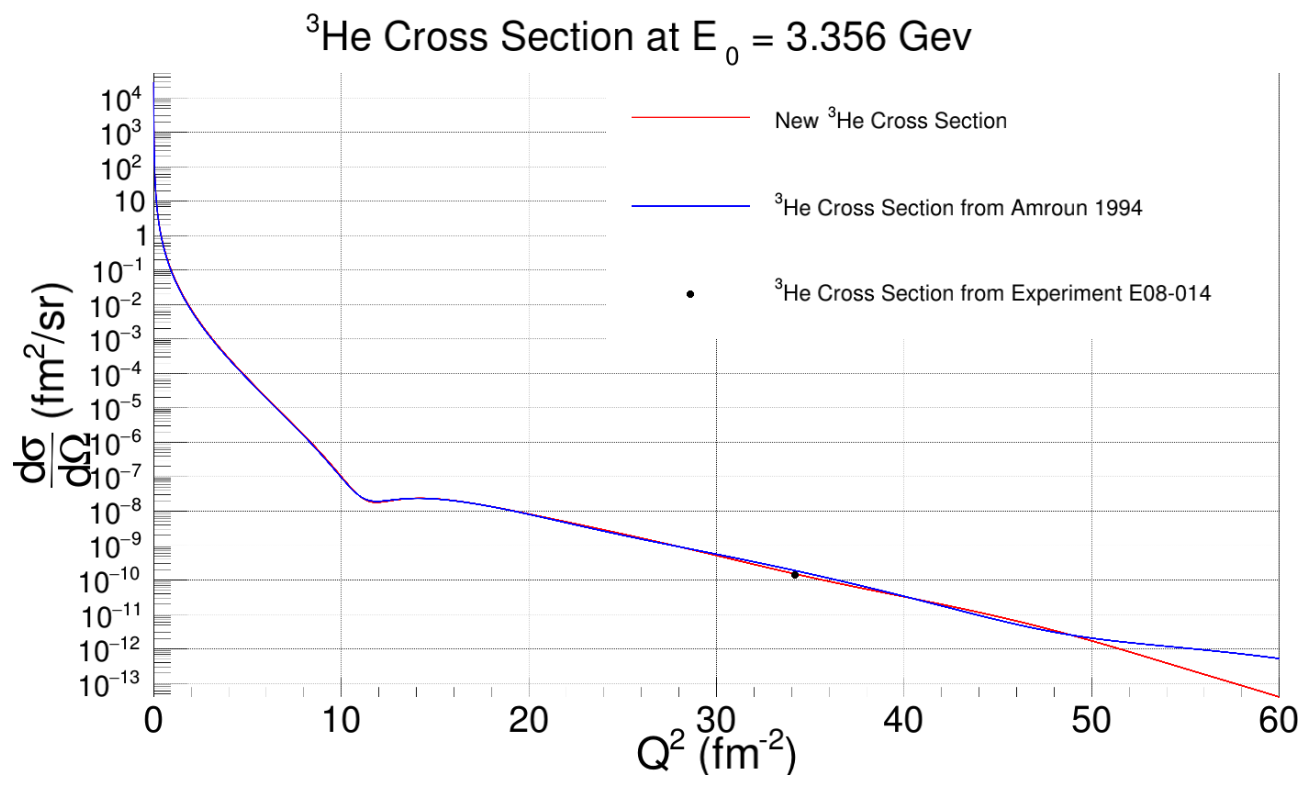

(a) Log Plot Zoomed Out.

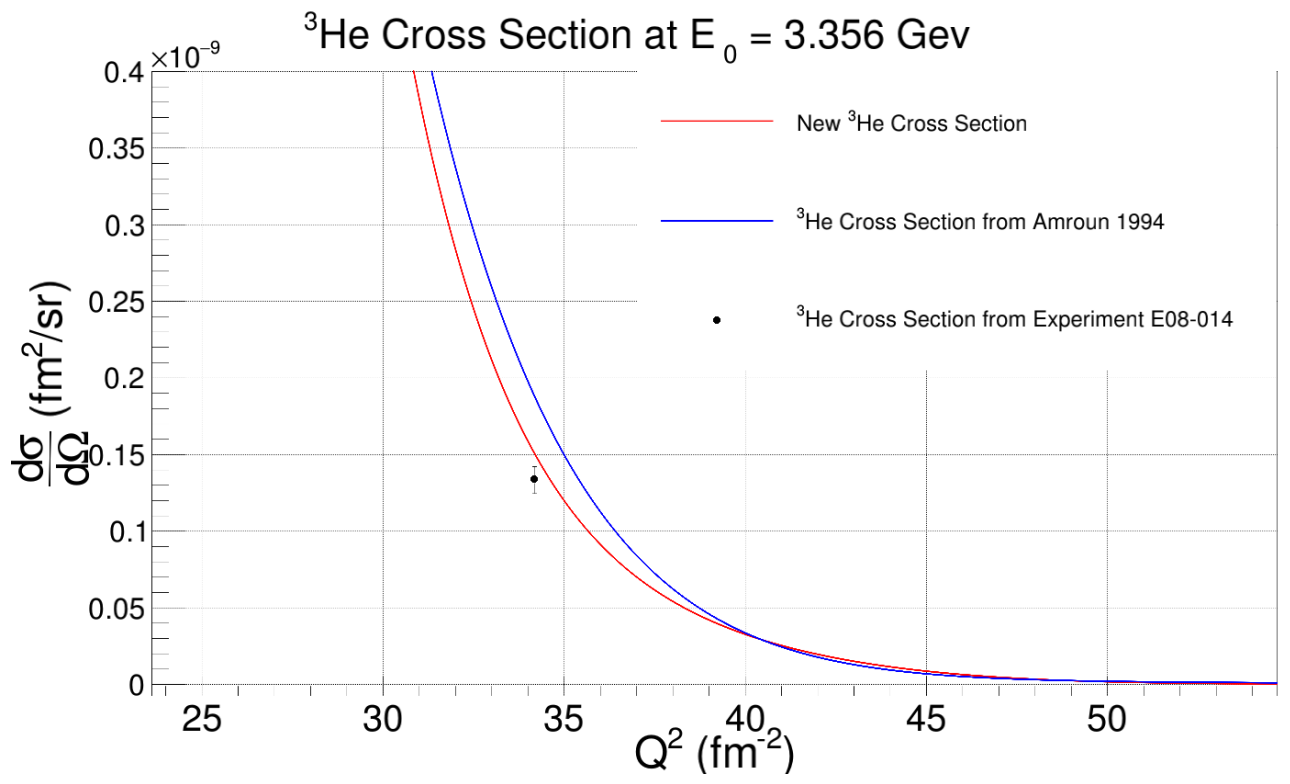

(b) No Log on $\boldsymbol{Y}$-axis and Zoomed In.

Figure 4.13: ${ }^{3}$ He Elastic Cross Section. The upper plot is the ${ }^{3} \mathrm{He}$ elastic cross section using the kinematics of experiment E08-014 with the $Y$-axis on a log scale. The red line represents the new ${ }^{3} \mathrm{He}$ cross section fit using the new representative form factor fits from this work, and the blue line is the cross section built from the previous form factor fits found in [29]. The black dot represents the new ${ }^{3} \mathrm{He}$ cross section measurement from this analysis. The lower plot depicts the same functions as the upper plot, but it removes the log on the $Y$-axis and zooms in on the cross section measurement from this analysis. 


\subsubsection{Revisiting the E08-014 Cross Section}

Now that we have obtained a new representative fit for the expanded set of world data it is prudent to revisit the cross section extracted in Chapter 3 . To find the elastic cross section for ${ }^{3} \mathrm{He}$ presented earlier the elastic electrons were simulated with the Monte Carlo SIMC. This Monte Carlo had a previous fit for the ${ }^{3} \mathrm{He}$ cross section from 29. We now have a fit that incorporates more high $Q^{2}$ data in the region of this analysis' cross section measurement. Let us now replace the previous fit in SIMC with our new fit and recalculate the cross section.

Once again the cross section in SIMC will be scaled by a constant value until the elastic electron yield from simulation matches that of the experiment. SIMC will then produce the average cross section for our ${ }^{3} \mathrm{He}$ data point. Since the shape of the two fits should be similar, and the cross section clearly does not change based on the fit used, we expect to find a very similar cross section value with the new fit compared to the value from the previous fit. Matching the electron yields requires scaling the SIMC cross section by a constant value of $C_{S I M C}=1.23197$. This is a larger scale factor than previously and is due to the decreased magnitude of $F_{m}$ in the new fit at this point's $Q^{2}$ of $34.19 \mathrm{fm}^{-2}$. The cross section yielded by SIMC after the yields are matched is found to be $1.345 \times 10^{-6} \mu \mathrm{b} / \mathrm{sr} \pm 0.086 \times 10^{-6} \mu \mathrm{b} / \mathrm{sr}$. This is in extremely close agreement (better than $1 \%$ ) with the previous fit's cross section of $1.335 \times 10^{-6}$ $\mu \mathrm{b} / \mathrm{sr} \pm 0.086 \times 10^{-6} \mu \mathrm{b} / \mathrm{sr}$ as we expected.

\subsection{5 $\quad{ }^{3}$ H Fits}

Now that we have selected $N_{\text {Gaus }}=8$ for ${ }^{3} \mathrm{H}$ we can run several hundred fits with the pseudorandom starting $R_{i}$ values along with the $R_{i}$ optimization procedure. The initial spacing of the $R_{i}$ values for these fits was $R_{0}=0.2-0.3, R_{1-4}=0.5-0.6$, and $R_{5-7}=0.8-0.9$ as explained in Section 4.3.1. A total of 2600 individual fits using the pseudorandom $R_{i}$ values were generated. Table 4.5 shows the results of these 2600 fits without any $\chi_{\max }^{2}$ cut and with a $\chi^{2}$ maximum of 603 in the same fashion as Tables 
4.3 and 4.4. 908 fits survive the $\chi^{2}$ cut of 603 , and the remaining fits have charge form factors with the desired sharp minima.

\begin{tabular}{|ccccccccc|}
\hline $\boldsymbol{N}_{\text {Gaus }}$ & Avg. $\chi^{\mathbf{2}}$ & $\chi_{\boldsymbol{r}}^{\mathbf{2}}$ & BIC & AIC & $\sum \boldsymbol{Q}_{\boldsymbol{i}_{\text {ch }}}$ & $\sum \boldsymbol{Q}_{\boldsymbol{i}_{\boldsymbol{m}}}$ & $\boldsymbol{\chi}_{\text {max }}^{\mathbf{2}}$ & $\begin{array}{c}\text { Below } \\
\text { Cut }\end{array}$ \\
\hline 8 & 611.4 & 2.817 & 266.2 & 238.5 & 1.089 & 1.335 & No Cut & 2600 \\
8 & 601.8 & 2.773 & 264.7 & 237.1 & 1.090 & 1.329 & 603 & 908 \\
\hline
\end{tabular}

Table 4.8: Metrics for Final ${ }^{3} \mathbf{H}$ Fits. $N_{\text {Gaus }}$ is the number of Gaussians used in the SOG fits, Avg. $\chi^{2}$ is the average $\chi^{2}$ value for the fits, $\chi_{r}^{2}$ is the average reduced $\chi^{2}$ value for the fits, BIC is the average Bayesian information criterion value of the fits, AIC is the average Akaike information criterion value of the fits, $\sum Q_{i_{c h}}$ is the average sum of the $Q_{i_{c h}}$ fit parameters, $\sum Q_{i_{m}}$ is the average sum of the $Q_{i_{m}}$ fit parameters, $\chi_{\max }^{2}$ is the maximum $\chi^{2}$ value for a fit to be deemed 'good', and 'Below Cut' is the number of fits that survived the $\chi_{\max }^{2}$ cut.

Table 4.8 shows that the $\chi_{\max }^{2}$ cut again improves all of the metrics as expected. Of the 2600 fits 908, 35\%, survive the $\chi_{\max }^{2}$ cut. This shows that the fits have more difficulty converging than they did for ${ }^{3} \mathrm{He}$, but they still converge with enough regularity to get a useful and reliable sample of fits. There are 234 data points for ${ }^{3} \mathrm{H}$ resulting in a $\chi^{2}$ of 601.8 . This works out to a $\chi^{2}$ of 2.572 per data point. This value is significantly higher than the value for ${ }^{3} \mathrm{He}$ of 1.686 implying that the data for ${ }^{3} \mathrm{H}$ is not as robust. Later in this section we will see that much of this larger $\chi^{2}$ is due to disagreement between the various experimental measurements. If the individual datasets were given a floating normalization the $\chi^{2}$ value would decrease significantly, but this would mask the actual state of the ${ }^{3} \mathrm{H}$ world data. The fits still look very reasonable and agree well with past results.

The $\sum Q_{i_{c h}}=1.090$ when we expect it to equal unity from physical considerations of the form factors discussed in Section 1.3. This means the fits on average see $9 \%$ more electric charge than expected. Again, this occurs because we did not force the form factors to approach unity at the origin, but this demonstrates our relatively worse understanding of the charge form factor for ${ }^{3} \mathrm{H}$ compared to ${ }^{3} \mathrm{He}$. If we forced the form factors to unity at the origin they would artificially turn over even more at small radii. $\sum Q_{i_{m}}=1.329$ shows an excess of $32.9 \%$ in the magnetic charge which 
is much worse than the charge form factor for ${ }^{3} \mathrm{H}$ or either form factor for ${ }^{3} \mathrm{He}$. Now we see the analytical use of not forcing the $Q_{i}$ to sum to unity artificially. If we had done this it would not be nearly as clear that most of our uncertainty about the ${ }^{3} \mathrm{H}$ form factors comes from our lack of understanding of the magnetic form factor. This continues to stress the need for more high $Q^{2}$ and back angle data for ${ }^{3} \mathrm{H}$ to improve our understanding of the form factors.

Figure 4.14 shows the charge form factors from each of the 2600 fits from Table 4.8. Figure 4.14a shows the 2600 fits without any $\chi_{\max }^{2}$ cut plotted together, and Figure $4.14 \mathrm{~b}$ shows the 908 fits surviving a $\chi_{\max }^{2}=603$ cut. Plotted along with this analysis' fits in red is a blue line representing the average result from [29] in the range that analysis considered its fits to be valid. It is important to note that no new data has been added for ${ }^{3} \mathrm{H}$ so we do not expect our results to diverge much from past results like $[29]$. This makes the ${ }^{3} \mathrm{H}$ results more useful to check the consistency of our methodology with past results and as a point of comparison to the ${ }^{3} \mathrm{He}$ results. It was initially hoped that new ${ }^{3} \mathrm{H}$ data would be available for this analysis from the planned experiment E12-14-009 [55] which was the original basis for this thesis. Unfortunately, due to budget uncertainty and continuing resolutions this experiment was cancelled. Happily, Hall A was able to take some elastic ${ }^{3} \mathrm{H}$ data in the end, and once the data has been analyzed it should be a simple matter to add it to this analysis.

Looking at Figure $4.14 \mathrm{a}$ we notice that many of the fits are again behaving in a nonphysical manner. We observe numerous 'dip only' minima where the first sharp minimum is expected. These are even more shallow than the ${ }^{3} \mathrm{He}$ dips and often almost miss the first minima entirely. There are even two fits that seemingly failed to converge at all and stand out wildly from the others. The location of the first minima look to be in good agreement with previous measurements even without the nonphysical fits being removed. The second minima, however, is barely pinned down at all. It seems as though it could be located anywhere from $Q^{2}=26-60+\mathrm{fm}^{-2}$ with a denser grouping around $30 \mathrm{fm}^{-2}$. Some of the fits indicate the presence of a second minimum at much higher $Q^{2}$ based on the current data. 


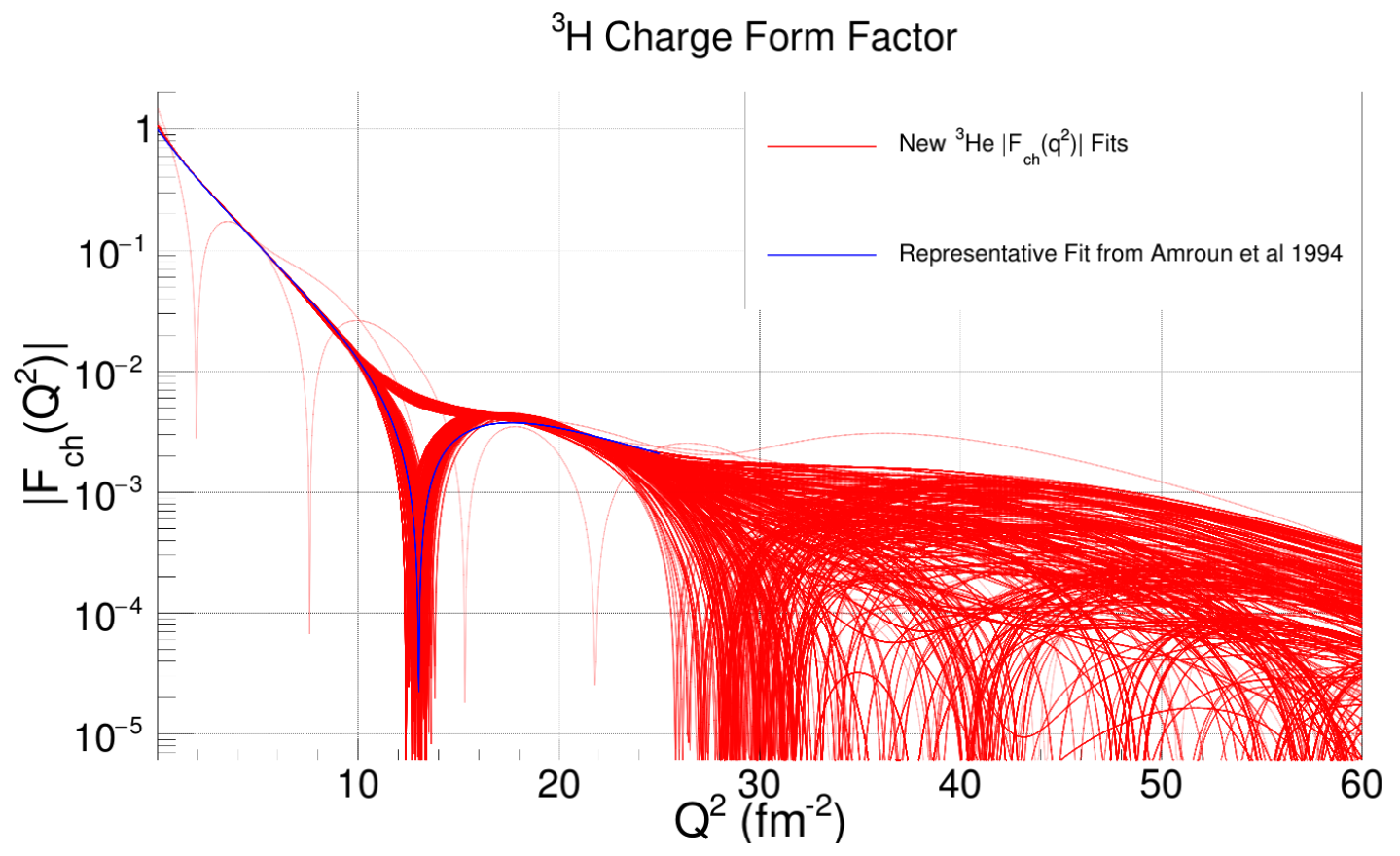

(a) Charge Form Factors from $2600{ }^{3} \mathrm{H}$ Fits with no $\chi_{\max }^{2}$ cut.

${ }^{3} \mathrm{H}$ Charge Form Factor

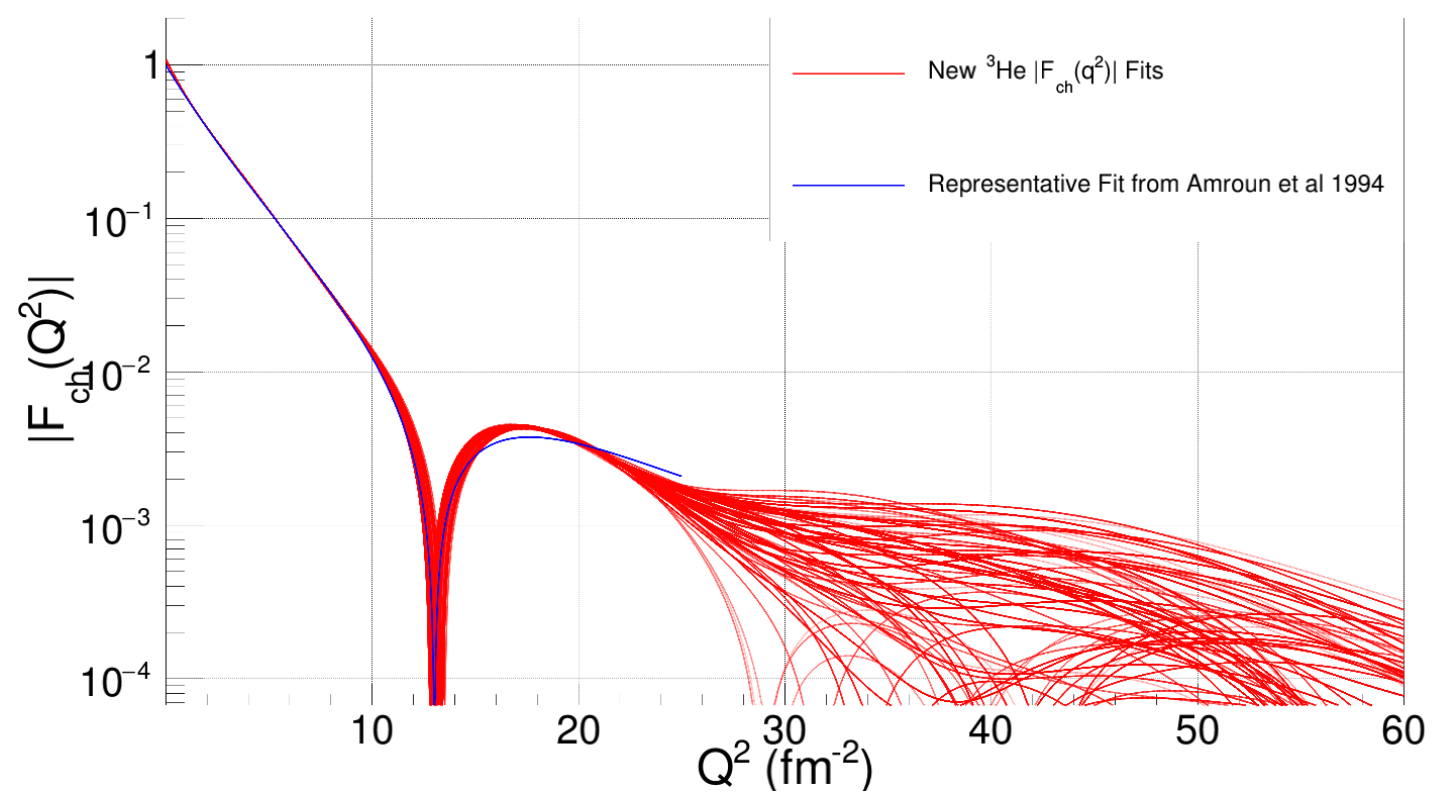

(b) Charge Form Factors from $908{ }^{3} \mathrm{H}$ Fits surviving a $\chi_{\max }^{2}=603$ cut.

Figure 4.14: ${ }^{3} \mathbf{H}$ Charge Form Factors. These charge form factors (red lines) were derived from 2600 fits of the ${ }^{3} \mathrm{He}$ world data using pseudorandom initial $R_{i}$ values and the $R_{i}$ optimization procedure. The blue line is the fit from [29]. 
Now we apply the $\chi_{\max }^{2}=603 \mathrm{cut}$, and get Figure $4.14 \mathrm{~b}$ with the resulting charge form factors that survive. Immediately it is obvious that the cut has removed most of the nonphysical and bizarre looking results. The results remain in reasonable agreement with [29 except the peak after the first minima is higher and falls off faster. The cluster of second minima near $30 \mathrm{fm}^{-2}$ has almost entirely disappeared. There is still no determination of the location of a secondary diffractive minima with the current data indicating it could be located anywhere from $Q^{2}=30-60+\mathrm{fm}^{-2}$ or not exist at all. There is simply not enough high precision data in the world data to make any determinations beyond $Q^{2} \approx 25 \mathrm{fm}^{-2}$.

Figure 4.15 shows the resulting magnetic form factors of each of the 2600 fits of the ${ }^{3} \mathrm{H}$ world data. Figure $4.15 \mathrm{a}$ shows the 2600 fits without any $\chi_{\max }^{2}$ cut plotted together, and Figure $4.15 \mathrm{~b}$ shows the 908 fits surviving the $\chi_{\max }^{2}=603$ cut. Beginning with the fits without a $\chi_{\max }^{2}$ cut in Figure $4.15 \mathrm{a}$ we see that, as with the charge form factor, there are many nonphysical fits. Most of these are 'dip only' minima. The fits are decently consistent overall, and the location of the first minima is consistent with past results.

Next we examine the magnetic form factors of the 908 fits of the ${ }^{3} \mathrm{H}$ world data that survive the $\chi_{\max }^{2}=603$ cut shown in Figure 4.15b. Once again, the cut has removed most of the nonphysical and bizarre looking results. After this cut our results still remain in agreement with [29]. This is as we expect since no new ${ }^{3} \mathrm{H}$ data was added to the world data. The fits indicate that a secondary diffractive minima could be anywhere from $Q^{2}=36-60+\mathrm{fm}^{-2}$ and offer little insight beyond this range. Again, the magnetic form factor is less well determined than the charge form factor fits due to the relative lack of high $Q^{2}$ and back angle data, and collecting more of this data is the best way to improve our knowledge of the ${ }^{3} \mathrm{H}$ magnetic form factor.

Using the functions describing the form factors we can calculate the charge densities as discussed in Section 4.2. Figure 4.16 shows the charge densities for ${ }^{3} \mathrm{H}$ prior to the $\chi_{\max }^{2}$ cut's application in $4.16 \mathrm{a}$ as well as the charge density for ${ }^{3} \mathrm{H}$ with a $\chi_{\max }^{2}$ cut $=603 \mathrm{in} 4.16 \mathrm{~b}$. Without the cut the charge density appears to be bundled in two 


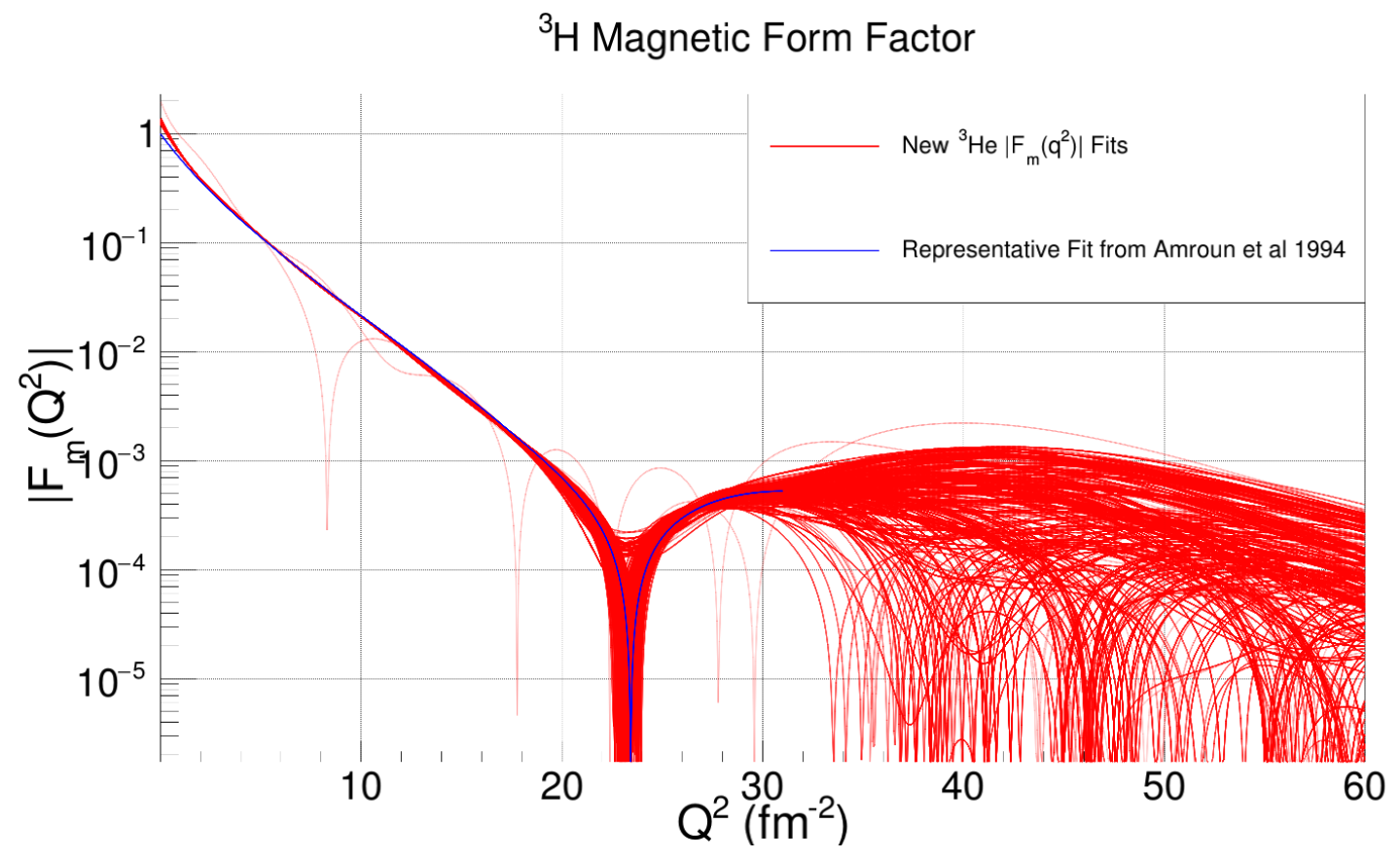

(a) Magnetic Form Factors from $2600{ }^{3} \mathrm{H}$ Fits with no $\chi_{\max }^{2}$ cut.

${ }^{3} \mathrm{H}$ Magnetic Form Factor

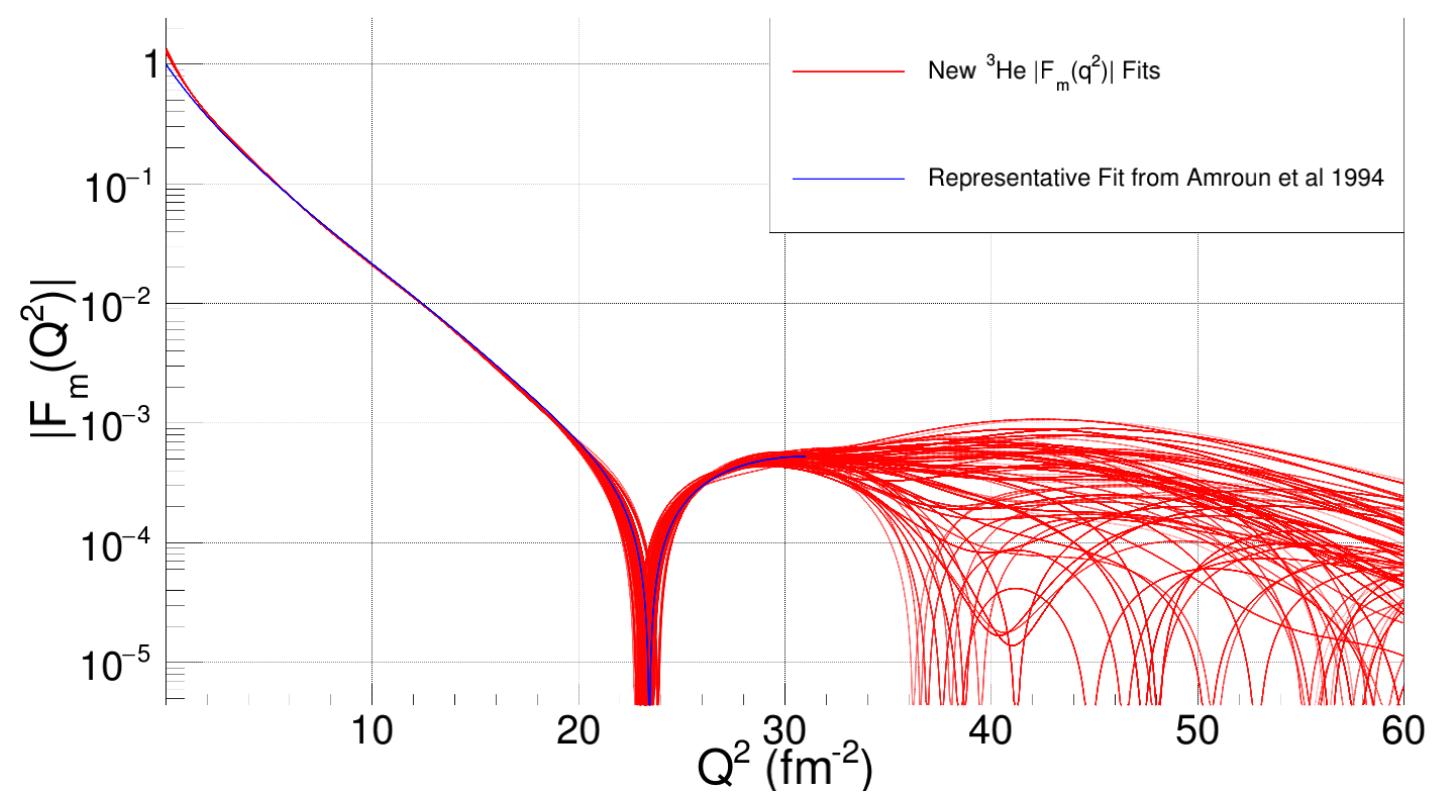

(b) Magnetic Form Factors from $908{ }^{3} \mathrm{H}$ Fits surviving a $\chi_{\max }^{2}=603$ cut.

Figure 4.15: ${ }^{3} \mathbf{H}$ Magnetic Form Factors. These Magnetic form factors (red lines) were derived from 2600 fits of the ${ }^{3} \mathrm{He}$ world data using pseudorandom initial $R_{i}$ values and the $R_{i}$ optimization procedure. The blue line is the fit from [29]. 
different groups, one predicting a higher density at a radius of zero and one predicting a lower density at zero. There is even a single fit which failed to converge well centered around $2 \mathrm{fm}$. Each of these groups has a fairly wide spread of magnitudes at low radii. However, once the nonphysical fits are removed by the $\chi_{\max }^{2}$ cut the higher bundle of fits disappears leaving only the lower.

The shapes of the ${ }^{3} \mathrm{H}$ charge densities of the 'good' fits are fairly consistent, but where they reach the origin still has a much wider spread than ${ }^{3} \mathrm{He}$ 's charge density. Once again this is due to the dearth of data for ${ }^{3} \mathrm{H}$, especially at higher kinematics. Below a radius of about $0.5 \mathrm{fm}$ the charge density turns over and comes to to a plateau. This turnover occurs at smaller radii for fits with larger charge density at low radii. Again, the charge density tapers off by a radii of 5 fm justifying the guess of $R_{\max }$ in Section 4.3.1.

Using Equation 1.19 we can also calculate the RMS charge radii for each of the individual fits. Figure 4.17 shows a plot of the 2600 charge radii resulting from the fits of ${ }^{3} \mathrm{H}$ world data. Figure $4.17 \mathrm{a}$ shows the 2600 fits without a $\chi_{\max }^{2}$ cut, and $4.17 \mathrm{~b}$ shows the 908 fits surviving the $\chi_{\max }^{2}=603$ cut. The cut removes some of the radii at the edges of the distribution, but it does not significantly alter its overall shape. The average of all the RMS charge radii surviving the cut was $2.02 \mathrm{fm}$. A Gaussian fit to these radii finds a mean of $2.02 \mathrm{fm}$ with a standard deviation of $0.0133 \mathrm{fm}$. Recall that there is an additional uncertainty on this charge radius beyond the standard deviation due to the uncertainty on the $\sum Q_{i}$ as discussed in Section 4.3.3. This additional uncertainty for ${ }^{3} \mathrm{H}$ is larger than that of ${ }^{3} \mathrm{He}$ since the $\sum Q_{i_{c h}}$ for ${ }^{3} \mathrm{H}$ is approximately 1.089, but the $\sum Q_{i_{c h}}$ for ${ }^{3} \mathrm{He}$ was only 1.008 . The ${ }^{3} \mathrm{H}$ radii are less well grouped than the ${ }^{3} \mathrm{He}$ radii and form a less well defined Gaussian distribution, but they still appear to be clustered roughly around a mean value without errant peaks separated from the prime distribution. Note that the $R_{i}$ used for each fit were selected in discrete increments of $0.1 \mathrm{fm}$, but if the $R_{i}$ were chosen continuously a better Gaussian for the charge radii distribution might be obtained.

As was done with ${ }^{3} \mathrm{He}$, the parameters of the ${ }^{3} \mathrm{H}$ fits can be plotted for further 


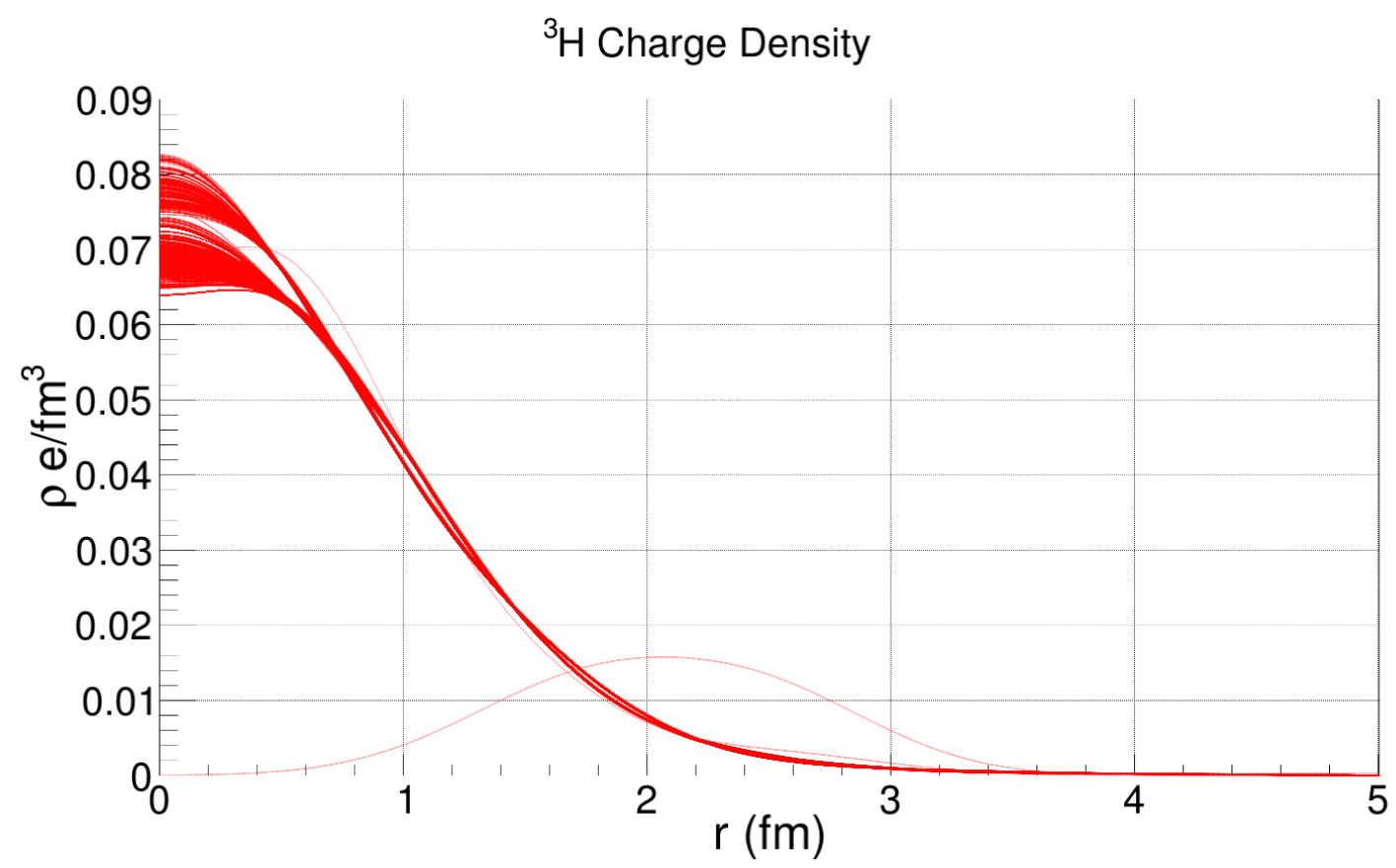

(a) Charge densities of $2600{ }^{3} \mathrm{H}$ fits with no $\chi_{\max }^{2}$ cut.

${ }^{3} \mathrm{H}$ Charge Density

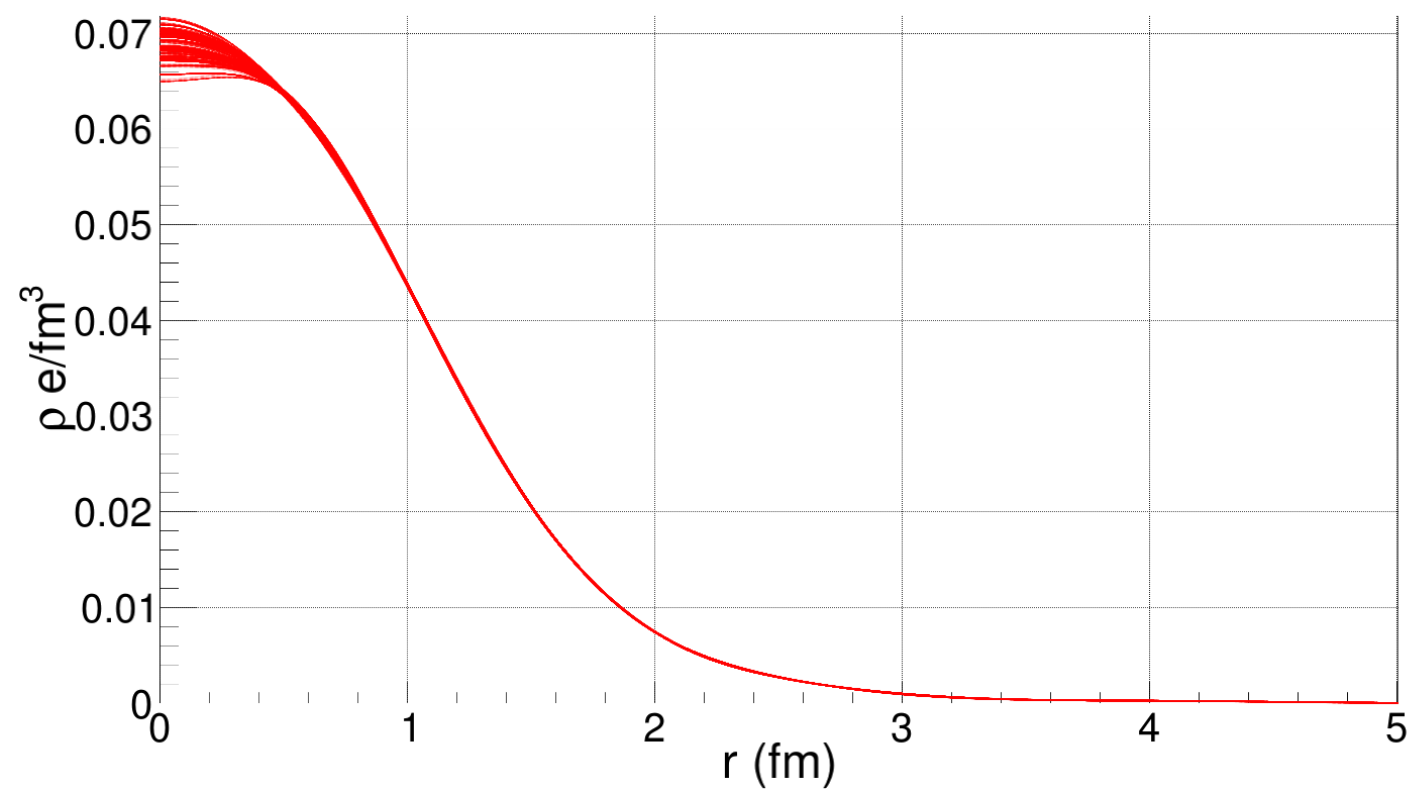

(b) Charge densities of $908{ }^{3} \mathrm{H}$ fits surviving a $\chi_{\max }^{2}=603 \mathrm{cut}$.

Figure 4.16: ${ }^{3} \mathbf{H}$ Charge Densities. The units of the $Y$-axis are the elementary electron charge per cubic fermi.

insight. Figure 4.18 shows the $Q_{i}$ fit parameters discussed in 4.2 with Figure $4.18 \mathrm{a}$ showing the $Q_{i_{c h}}$ parameters and Figure $4.18 \mathrm{~b}$ showing the $Q_{i_{m}}$ parameters. Each of 


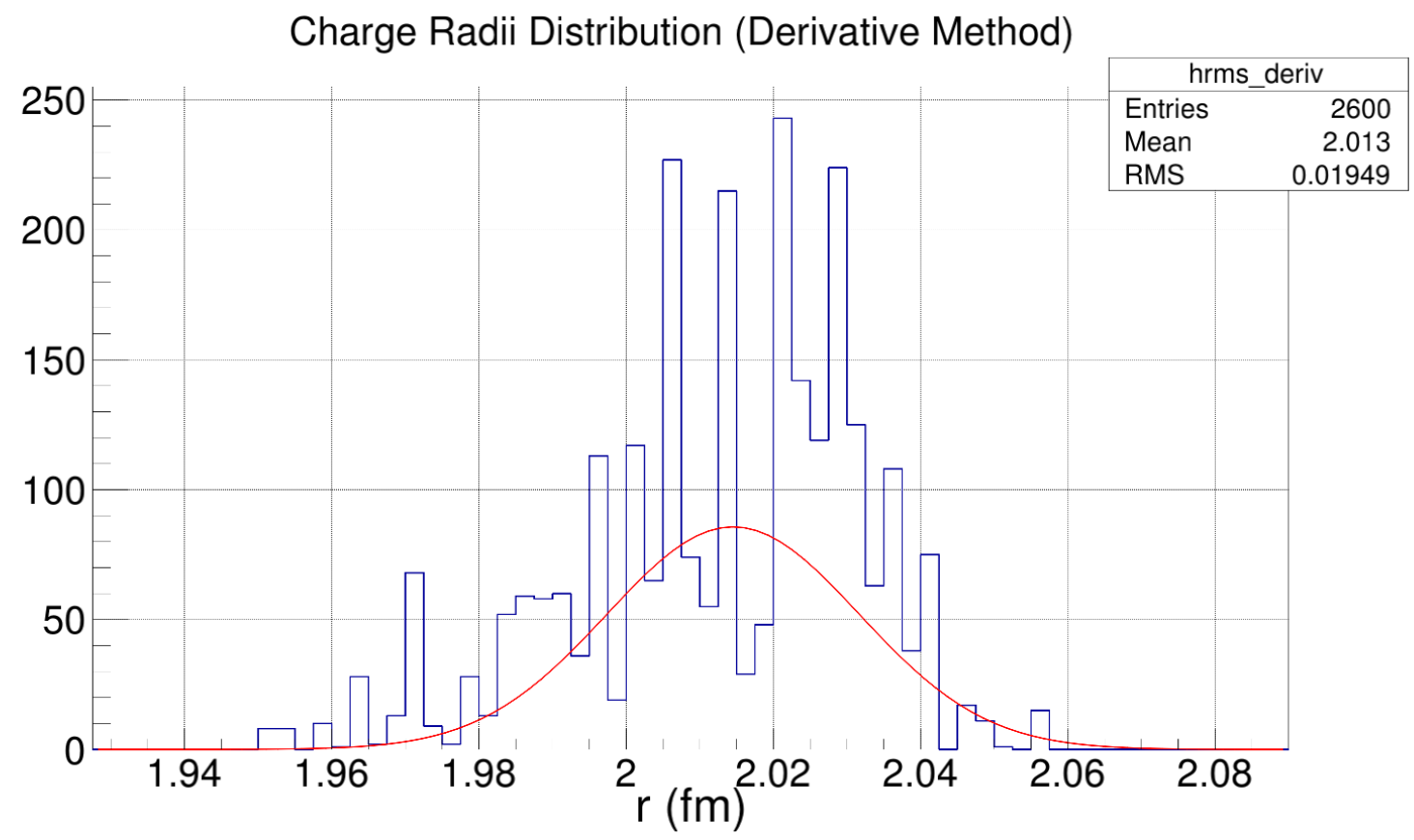

(a) RMS charge radii of $2600{ }^{3} \mathrm{H}$ fits with no $\chi_{\max }^{2}$ cut.

Charge Radii Distribution (Derivative Method)

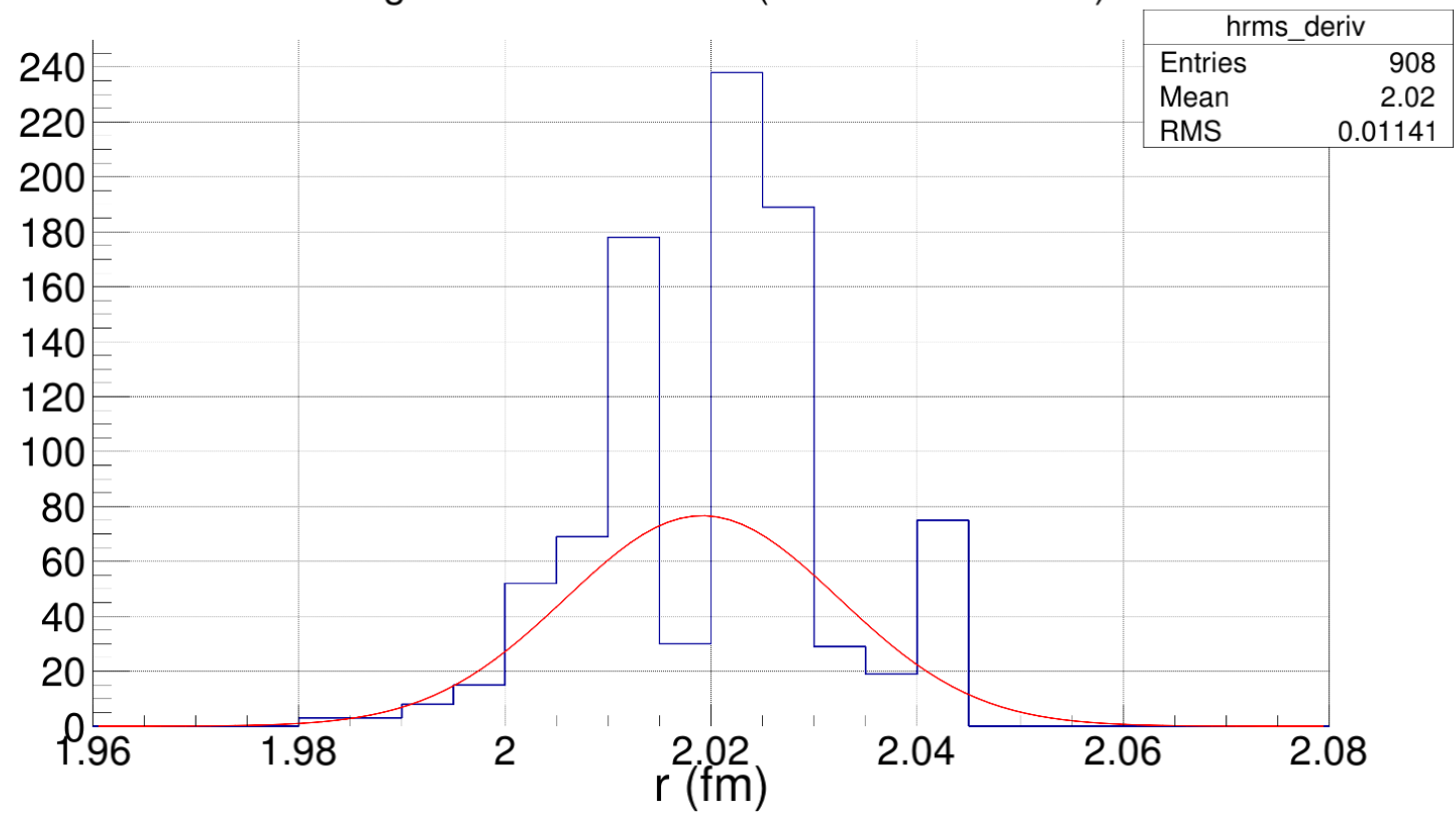

(b) RMS charge radii of $908{ }^{3} \mathrm{H}$ fits surviving a $\chi_{\max }^{2}=500$ cut.

Figure 4.17: ${ }^{3}$ H RMS Charge Radii.

the 8 parameters is shown up to a maximum value of 0.5 . As previously, the structure of the parameter plots did not vary significantly after the $\chi_{\max }^{2}$ cut was applied so only the plots with the cut are shown. The results are consistent with those of ${ }^{3} \mathrm{He}$ in 
Section 4.3 .3 in that the $Q_{i}$ values are larger for the inner Gaussians placed at smaller radii, and thus they have the strongest influence on the overall fits. The $Q_{i}$ are still strongly coupled to the $R_{i}$ distribution, or 'model', of each individual fit with wide spreads in the $Q_{i}$ parameter values, especially for the inner $Q_{i}$.

Plotted in Figure 4.19 are the $R_{i}$ values 4.19a and the separation between consecutive $R_{i}$ values $4.19 \mathrm{~b}$ of the fits of the ${ }^{3} \mathrm{H}$ world data in fm. The insights gleaned from these plots parallel those found for ${ }^{3} \mathrm{He}$ in Section 4.3 .3 . The $R_{i}$ values are well grouped indicating the fitting procedure is finding similar results regardless of the pseudorandom initial conditions. Again we see that $R_{\max } \approx 5 \mathrm{fm}$. Finally, the distance between consecutive $R_{i}$ values still fluctuates somewhat, but the separations tend to converge to a central value, and the separation between consecutive $R_{i}$ values roughly doubles for the larger half of the radii.

A representative fit for the ${ }^{3} \mathrm{H}$ form factors was selected in the same manner as the one selected for ${ }^{3} \mathrm{He}$ in Section 4.3 .3 . Table 4.9 and Table 4.10 show the parameters for the representative fit of the ${ }^{3} \mathrm{H}$ world data along with the metrics used to test the 'goodness' of the fit respectively. Figure 4.20 shows plots of the representative charge and magnetic form factors for the new ${ }^{3} \mathrm{H}$ world data fits (error bands were again created with [54]). The charge form factor error band is well determined past the first minimum until about $Q^{2} \approx 24 \mathrm{fm}^{-2}$ where it begins to expand. Above $Q^{2} \approx$ $24 \mathrm{fm}^{-2}$ there is alomost no knowledge of the shape or magnitude of $F_{c h}$ due to a lack of high $Q^{2}$ data. The magnetic form factor is fairly well determined through the first minimum and up to $Q^{2} \approx 30 \mathrm{fm}^{-2}$. As $F_{m}$ passes above $Q^{2} \approx 30 \mathrm{fm}^{-2}$ there is almost no information about the form factor. Once again, to improve the understanding of the ${ }^{3} \mathrm{H}$ form factors more high $Q^{2}$ and back angle data is required.

The representative fits for the ${ }^{3} \mathrm{H}$ form factors can also be plotted along with the world data and the previous fit from 29 as shown in Figure 4.9. Note that the Amroun fits are deemed valid only up to $25 \mathrm{fm}^{-2}$ and $31 \mathrm{fm}^{-2}$, for $F_{c h}$ and $F_{m}$ respectively, but were extended for the sake of comparison. The form factor points shown are derived from Rosenbluth separations performed by the experiments used for this analysis' 

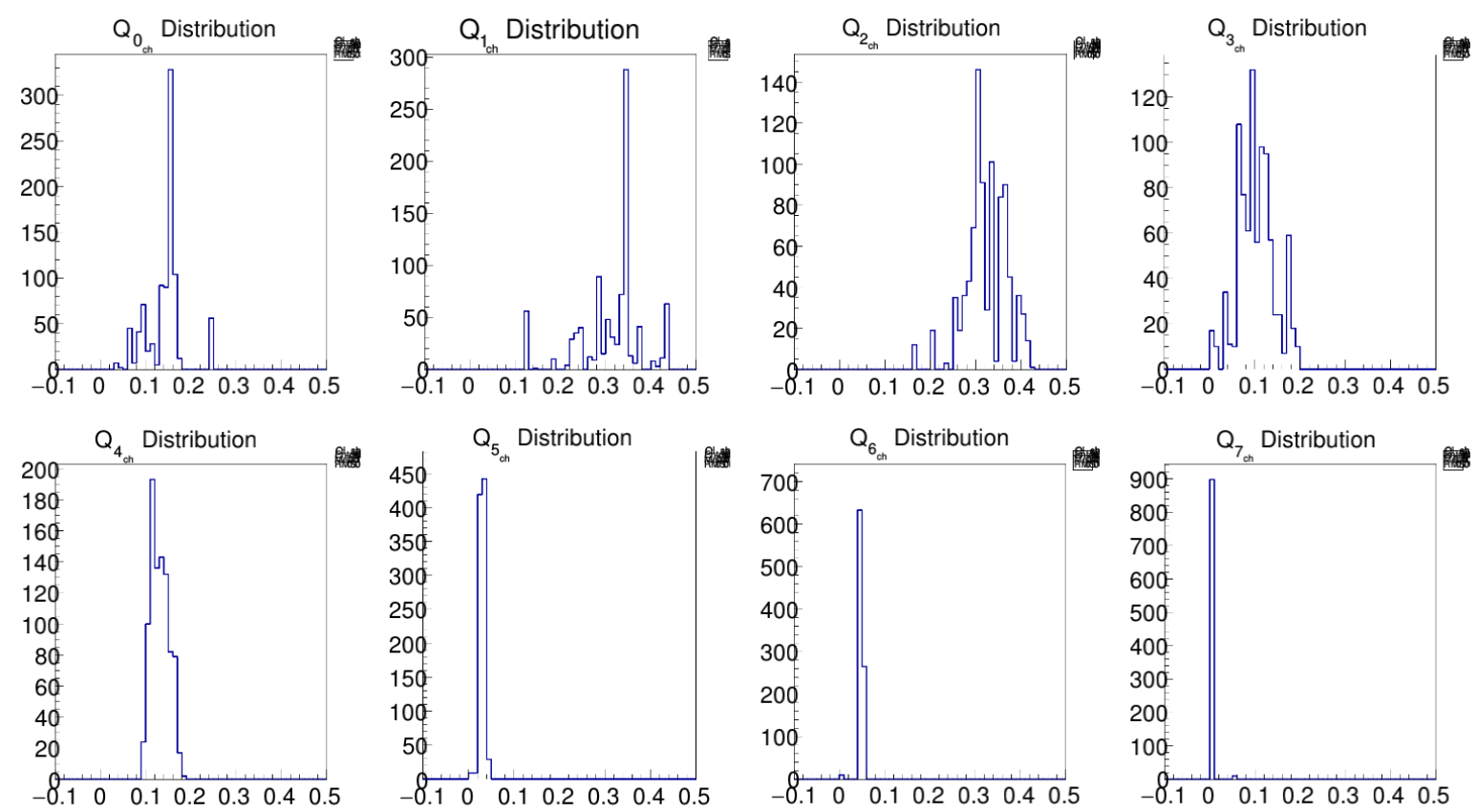

(a) $Q_{i_{c h}}$ parameters for ${ }^{3} \mathrm{H}$ with $\chi_{\max }^{2}=603$.
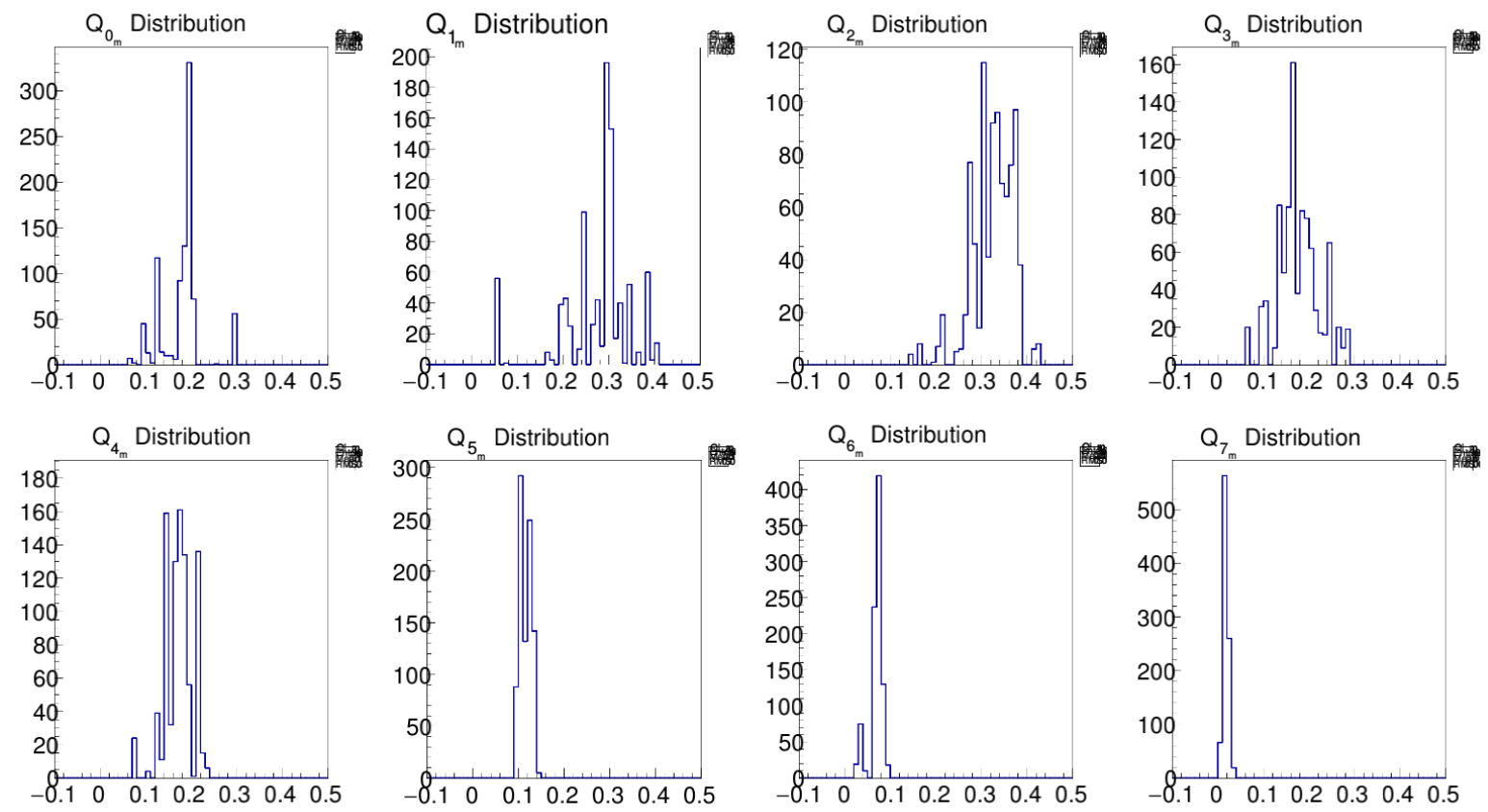

(b) $Q_{i_{m}}$ parameters for ${ }^{3} \mathrm{H}$ with $\chi_{\max }^{2}=603$.

Figure 4.18: ${ }^{3} \mathbf{H} \boldsymbol{Q}_{\boldsymbol{i}}$ Fit Parameters. The $X$-axis of these plots is the $Q_{i}$ value in the range of -0.1 to 0.5 . The $Y$-axis is the number of counts. The plots run from $Q_{0}$ up to $Q_{7}$. Notice that the distributions are each roughly centered about a specific value showing that the SOG fits are consistently finding similar $Q_{i}$ values. Also notice that the smaller radii parameters generally have larger values. 

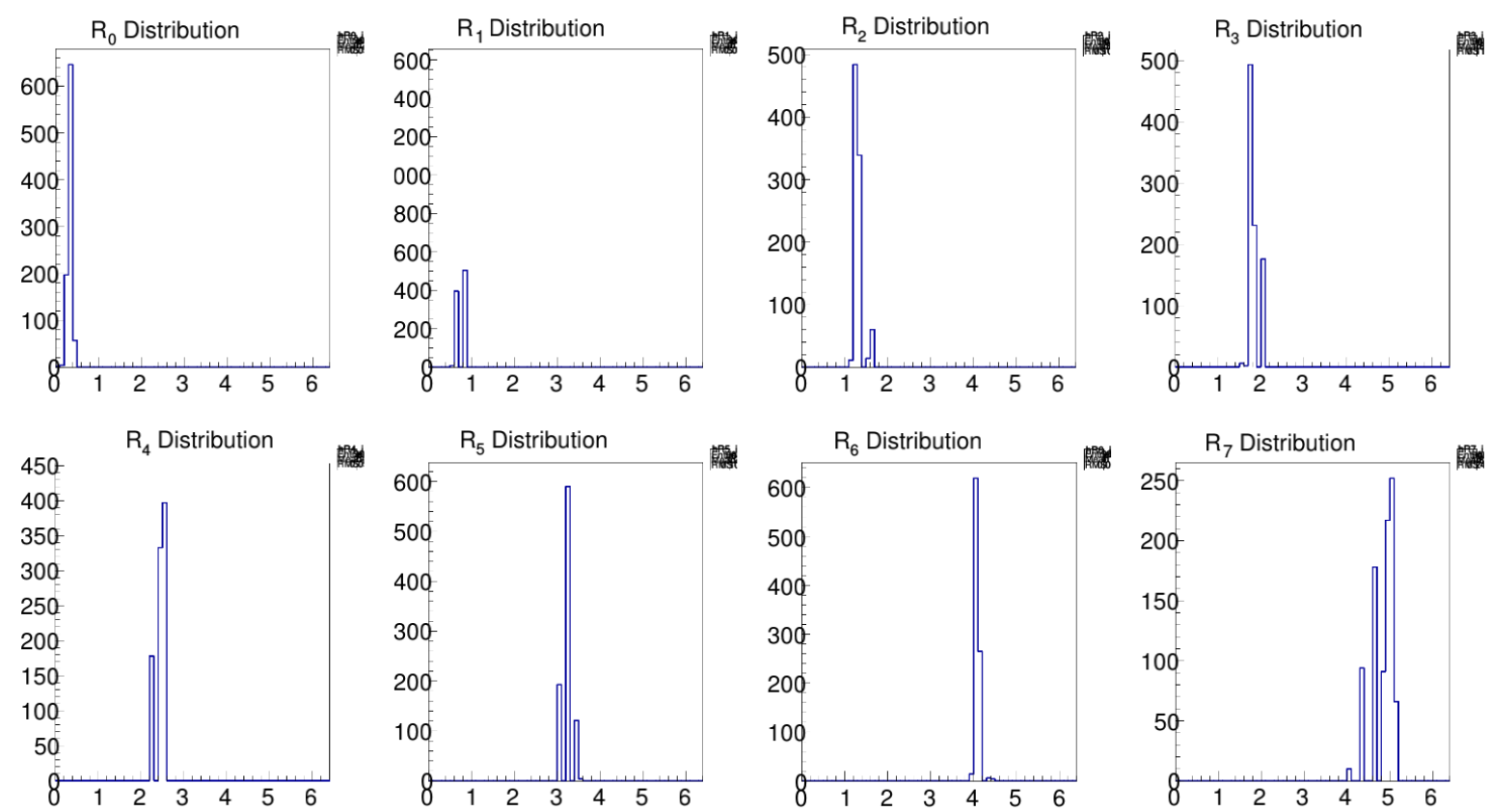

(a) $R_{i}$ values $(\mathrm{fm})$ of fits of ${ }^{3} \mathrm{H}$ world data $\chi_{\max }^{2}=500$.
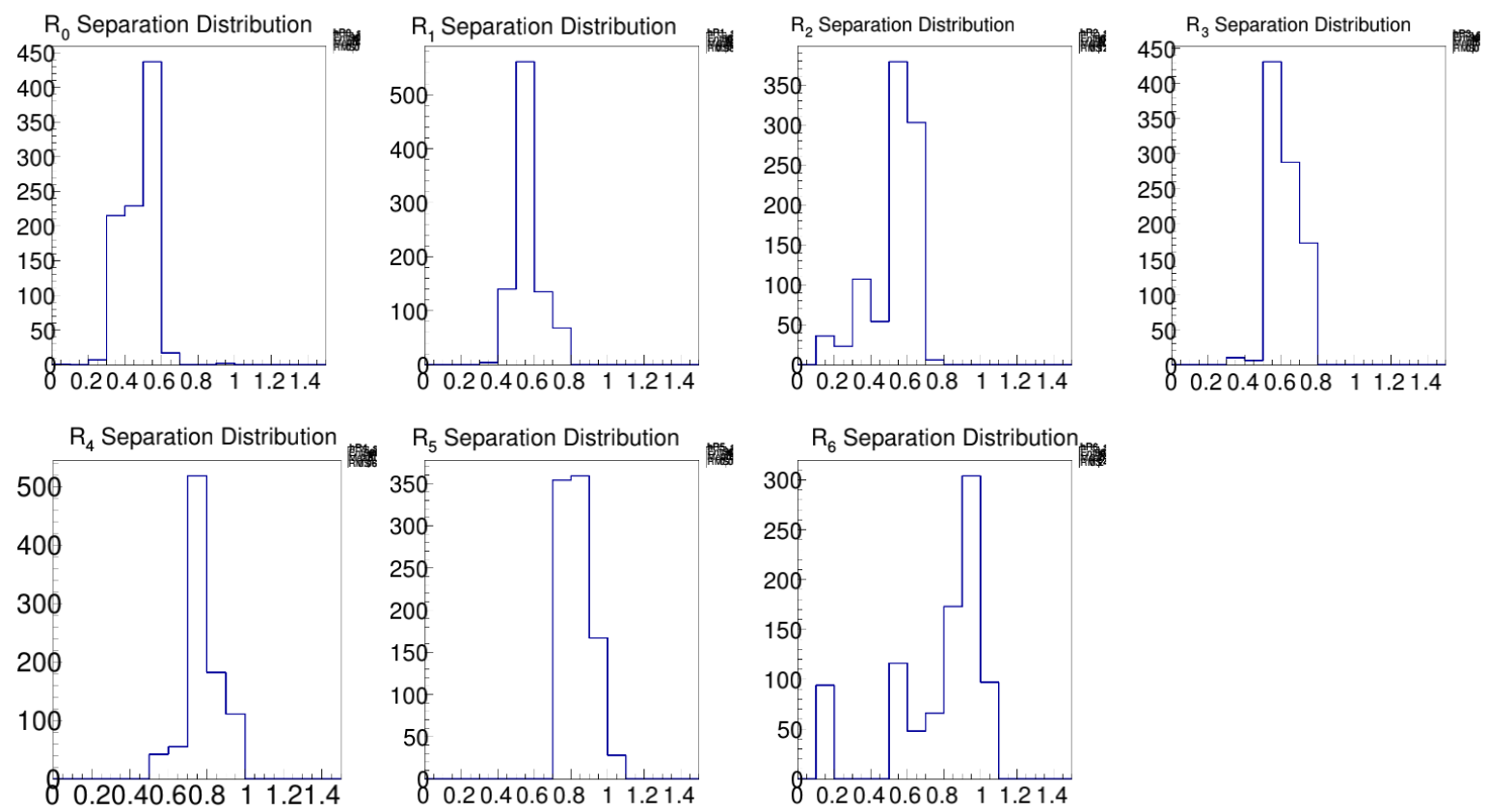

(b) Separation of consecutive $R_{i}$ values ( $\mathrm{fm}$ ) of fits of ${ }^{3} \mathrm{H}$ world data $\chi_{\max }^{2}=500$.

Figure 4.19: ${ }^{3} \mathbf{H e} \boldsymbol{R}_{\boldsymbol{i}}$ Values and Separations. The top plot shows the values for $R_{i}$ from $R_{0}$ up to $R_{7}$, and the bottom plot shows the distance separating each consecutive $R_{i}$, where the $X$-axes are in $\mathrm{fm}$. Notice that each plot is roughly centered about a specific value showing that the SOG fits are consistently finding similar $R_{i}$ values and separations. Also notice that the distributions widen at larger radii. 


\begin{tabular}{|ccll|}
\hline $\begin{array}{c}\text { Parameter } \\
\text { Number }\end{array}$ & $\boldsymbol{R}_{\boldsymbol{i}}(\mathbf{f m})$ & $\boldsymbol{Q}_{\boldsymbol{i}_{\boldsymbol{c h}}}$ & $\boldsymbol{Q}_{\boldsymbol{i}_{\boldsymbol{m}}}$ \\
\hline 1 & 0.3 & 0.151488 & 0.190646 \\
2 & 0.8 & 0.348372 & 0.301416 \\
3 & 1.4 & 0.29635 & 0.318972 \\
4 & 1.9 & 0.0978631 & 0.159433 \\
5 & 2.5 & 0.121983 & 0.173933 \\
6 & 3.3 & 0.0242654 & 0.106361 \\
7 & 4.1 & 0.049329 & 0.0665564 \\
8 & 4.8 & $4.40751 \times 10^{-11}$ & 0.0148866 \\
\hline
\end{tabular}

Table 4.9: Parameters for ${ }^{3} \mathbf{H}$ World Data Representative Fit. These fit parameters give the analytical form factors when plugged into Equation 4.4 .

\begin{tabular}{|ccccccc|}
\hline $\boldsymbol{N}_{\text {Gaus }}$ & Avg. $\chi^{\mathbf{2}}$ & $\chi_{r}^{\mathbf{2}}$ & BIC & AIC & $\sum \boldsymbol{Q}_{\boldsymbol{i}_{\text {ch }}}$ & $\sum \boldsymbol{Q}_{\boldsymbol{i}_{m}}$ \\
\hline 8 & 601.9 & 2.774 & 264.7 & 237.1 & 1.089 & 1.332 \\
\hline
\end{tabular}

Table 4.10: Metrics for ${ }^{3} \mathbf{H}$ World Data Representative Fit. $N_{\text {Gaus }}$ is the number of Gaussians used in the SOG fits, Avg. $\chi^{2}$ is the average $\chi^{2}$ value for the fits, $\chi_{r}^{2}$ is the average reduced $\chi^{2}$ value for the fits, BIC is the average Bayesian information criterion value of the fits, AIC is the average Akaike information criterion value of the fits, $\sum Q_{i_{c h}}$ is the average sum of the $Q_{i_{c h}}$ fit parameters, and $\sum Q_{i_{m}}$ is the average sum of the $Q_{i_{m}}$ fit parameters.

global fits. As discussed in Section 4.3 .3 there are far fewer discreet form factor points available as there have not been many Rosenbluth separations performed for ${ }^{3} \mathrm{H}$. Again, the Amroun data is not shown as it was not explicitly published. Notice that the new $F_{c h}$ fit once again agrees with the old fit and world data with some small shifts in magnitude beyond the first minimum. Above $Q^{2} \approx 30 \mathrm{fm}^{-2}$ the $F_{c h}$ fits start to diverge from a lack of data. The new $F_{m}$ fit agrees quite well with both the world data and old fit until $Q^{2} \approx 30 \mathrm{fm}^{-2}$ where all of the fits start to diverge and beyond which we have little knowledge.

Our understanding of the form factors in different kinematic ranges depends on the ${ }^{3} \mathrm{H}$ cross section world data distribution seen in Figure 4.22, Looking at the distribution of the world data it becomes clear that most of the data exists below $Q^{2}$ $\approx 10 \mathrm{fm}^{-2}$ and is sparse above there tapering off completely around $Q^{2} \approx 32 \mathrm{fm}^{-2}$. As with ${ }^{3} \mathrm{He}$, the dearth of high $Q^{2}$ and back angle ${ }^{3} \mathrm{H}$ elastic scattering data is restricting our understanding of the form factors. 


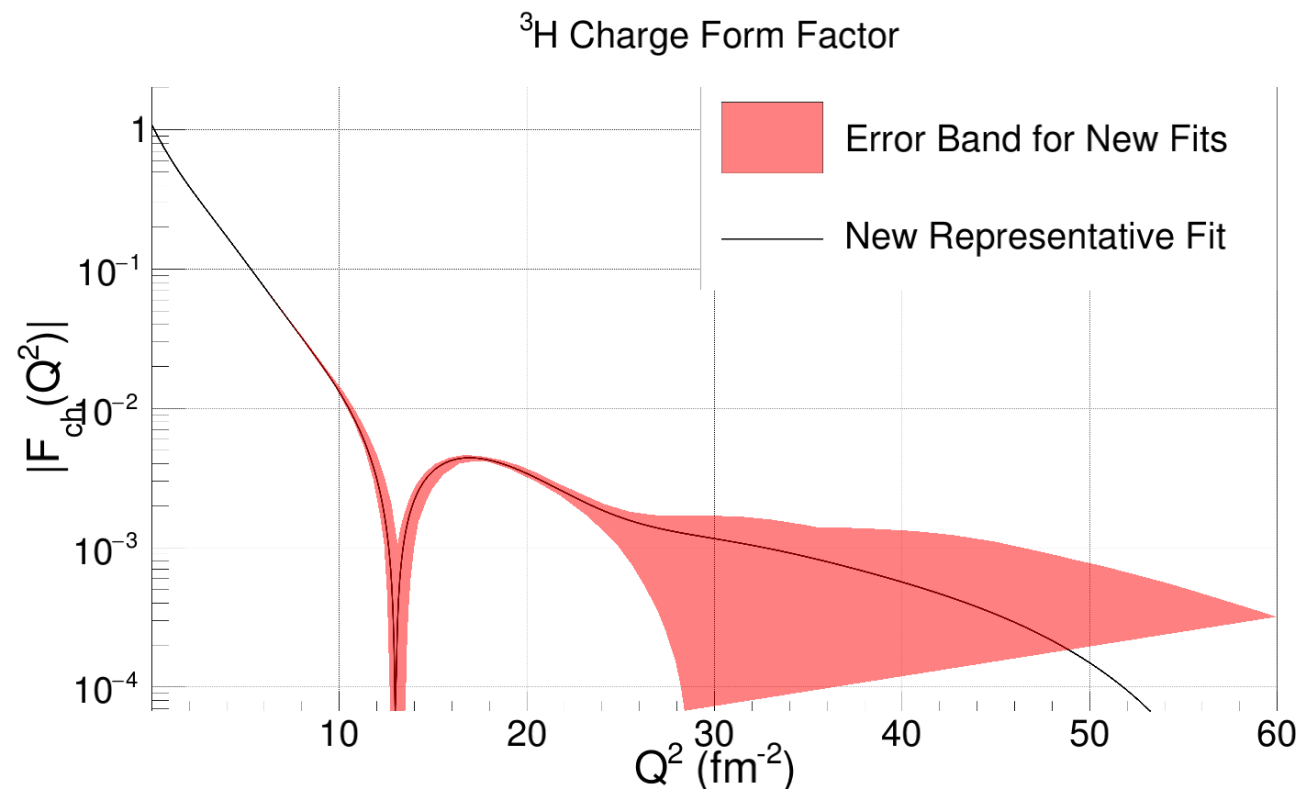

(a) ${ }^{3} \mathbf{H}$ Charge Form Factor for the New Representative Fit of World Data. ${ }^{3} \mathrm{H}$ Magnetic Form Factor

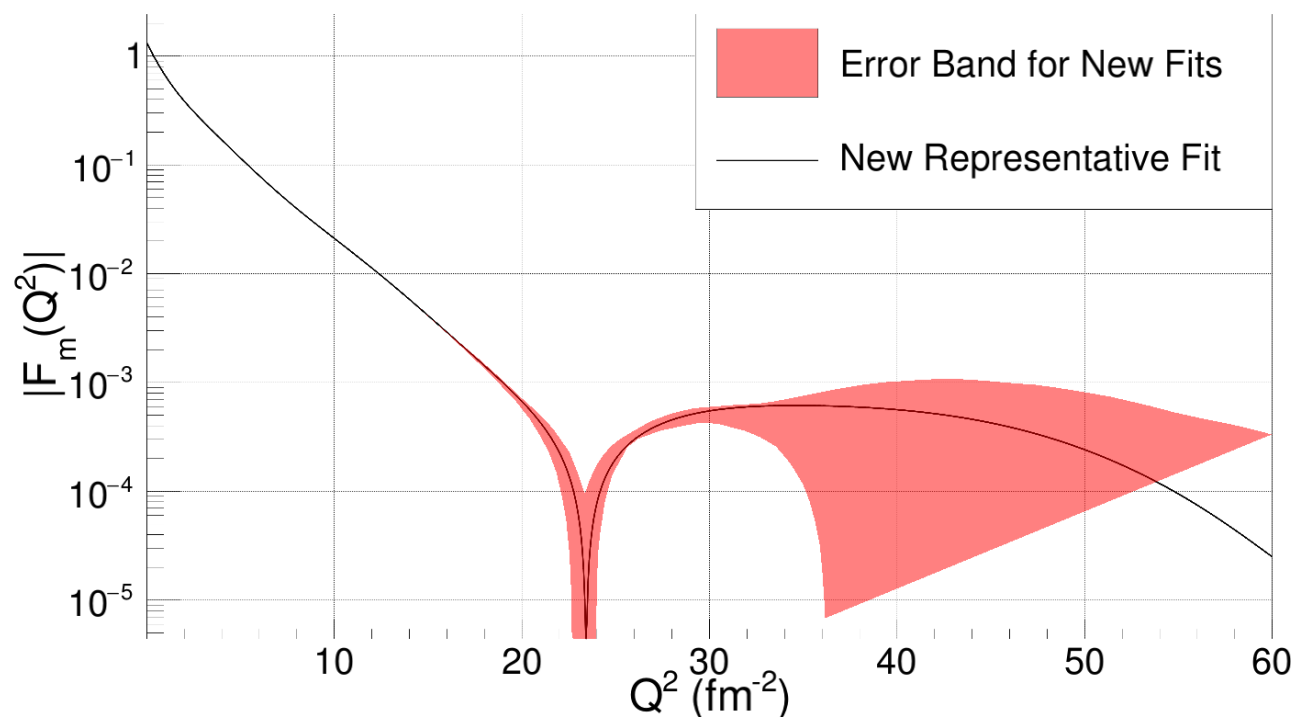

(b) ${ }^{3} \mathbf{H}$ Magnetic Form Factor for the New Representative Fit of World Data.

Figure 4.20: ${ }^{3} \mathbf{H}$ Form Factors for the New Representative Fit of World Data. The selected representative fit is shown in black and its error band is shaded in red.

This representative fit has a total $\chi^{2}$ of 601.9 for $234{ }^{3} \mathrm{H}$ elastic cross sections. This results in an average $\chi^{2}$ per point of 2.572 . Recall that this analysis did not float the normalization of the different datasets leading to a larger $\chi^{2}$. Figure 4.23 shows a plot of $\chi^{2}$ versus $Q^{2}$ for each data point in each dataset in the global fits. The highest $Q^{2}$ 


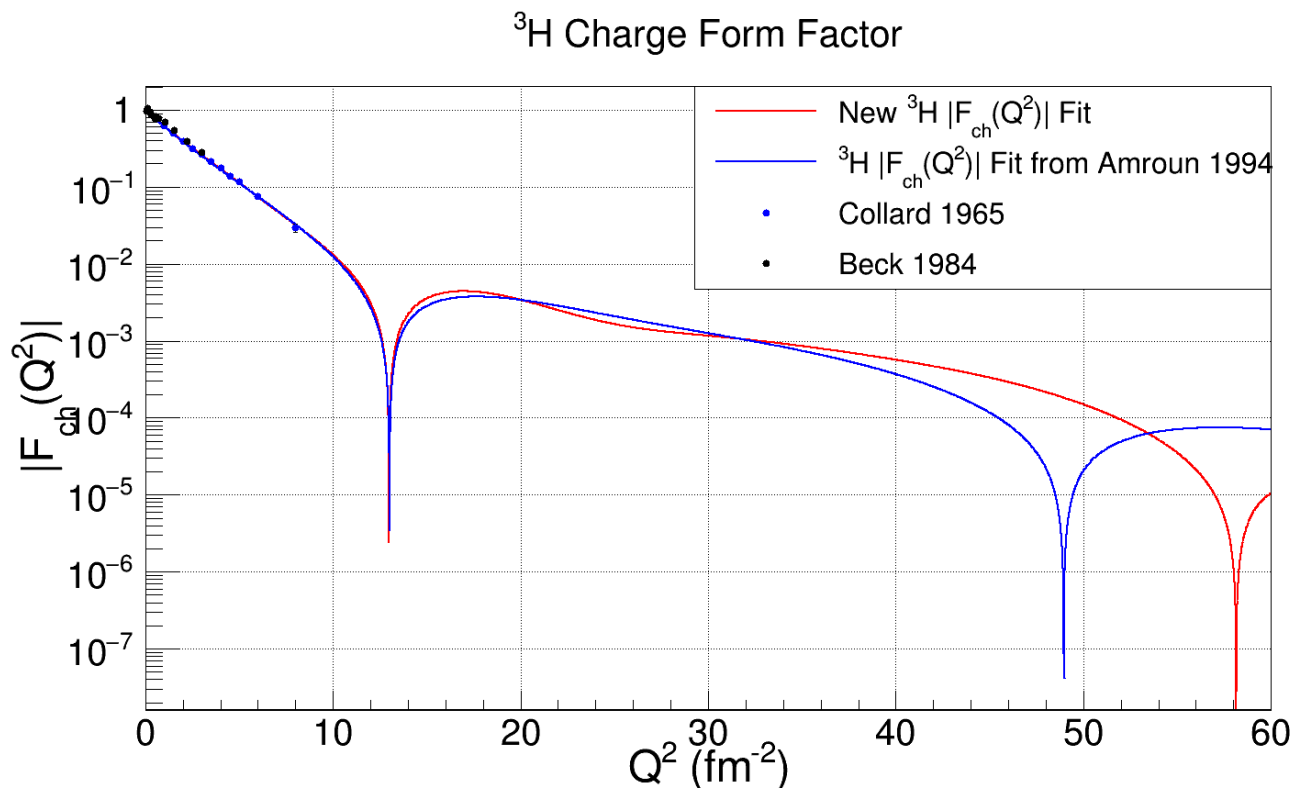

(a) ${ }^{3}$ H Representative Charge Form Factor with World Data and Amroun Fit ${ }^{3} \mathrm{H}$ Magnetic Form Factor

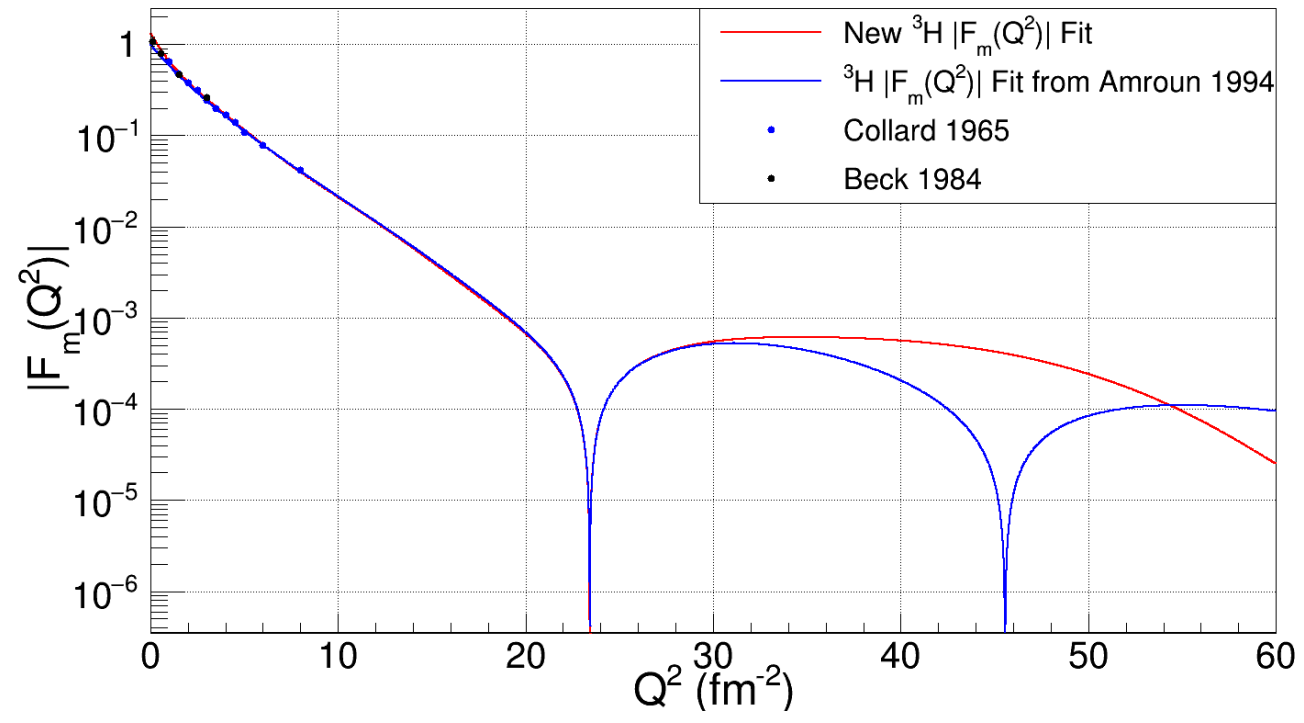

(b) ${ }^{3}$ H Representative Magnetic Form Factor with World Data and Amroun Fit.

Figure 4.21: ${ }^{3}$ H Representative Form Factors with World Data and Amroun Fit. The red lines are the new representative fits. The blue lines are the previous Amroun fits. Note that the Amroun fits are deemed valid only up to $25 \mathrm{fm}^{-2}$ and 31 $\mathrm{fm}^{-2}$, for $F_{c h}$ and $F_{m}$ respectively, but were extended for the sake of comparison. The points represent published form factor measurements from the datasets used for this analysis' global fits. These points were extracted via standard Rosenbluth separations. 


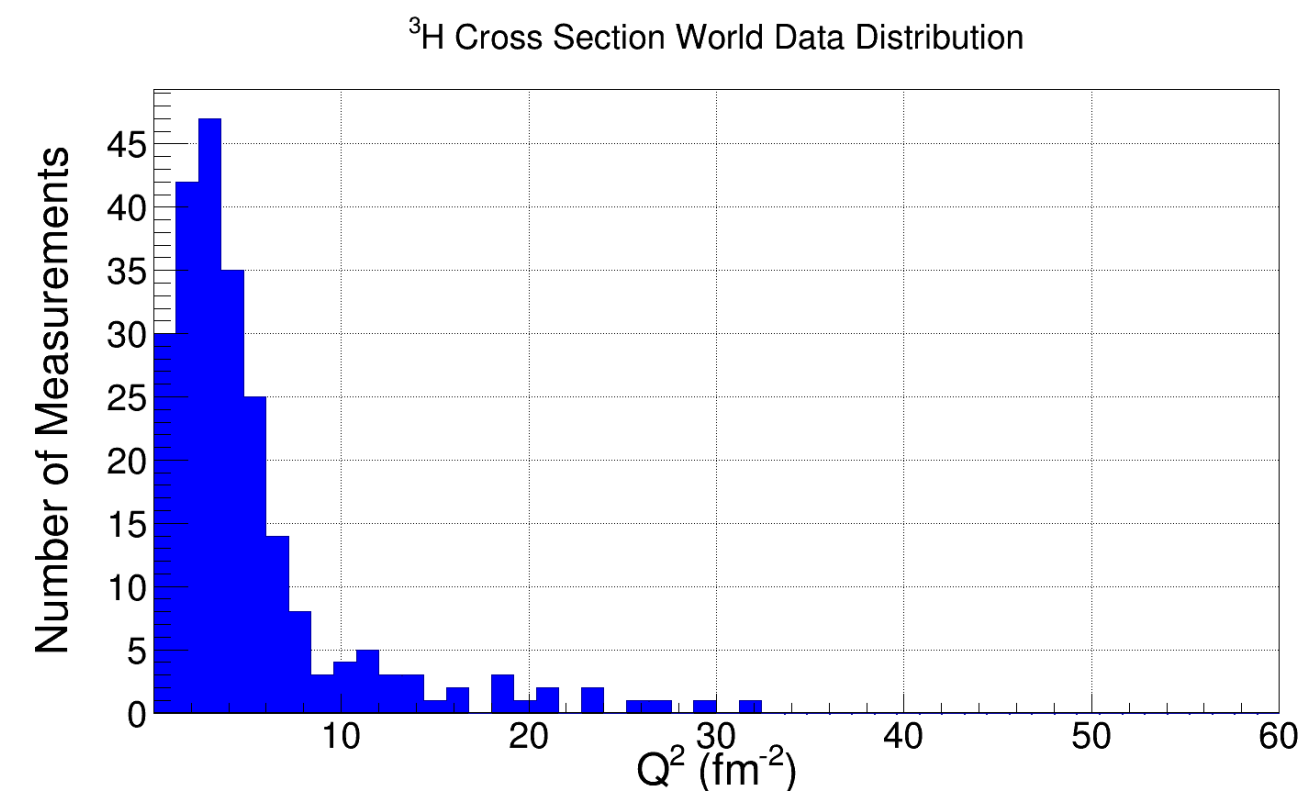

Figure 4.22: ${ }^{3} \mathbf{H}$ Cross Section World Data Distribution. This plot shows the ${ }^{3} \mathrm{H}$ cross section world data distribution in $Q^{2}$ used in this analysis.

data points come from the older datasets of Collard and Beck. It is interesting to note that there seems to be some structure to the Amroun dataset which will be discussed more shortly.

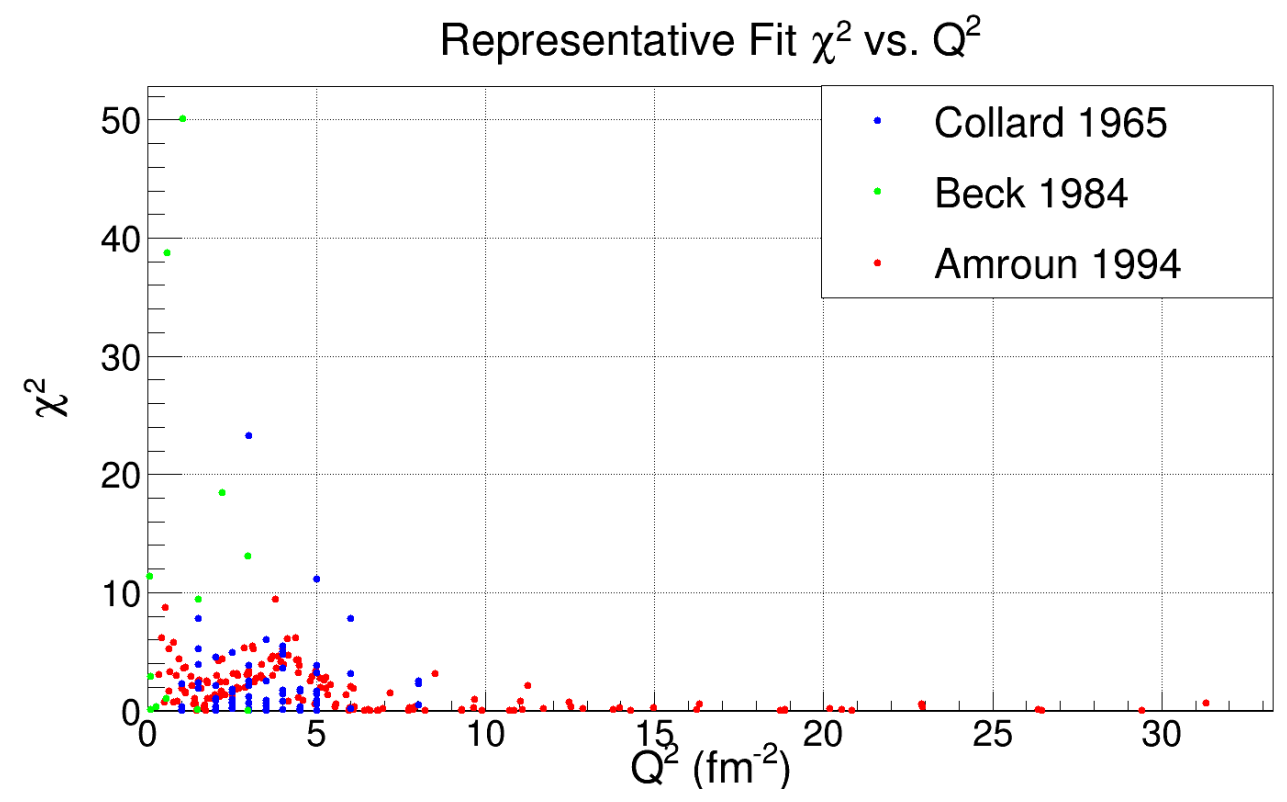

Figure 4.23: ${ }^{3} \mathbf{H}$ Representative Fit $\chi^{2}$ vs. $\boldsymbol{Q}^{\mathbf{2}}$. 
The plot of the representative fit's residual, given by Equation 4.13 , is shown in Figure 4.24. The residual plot for ${ }^{3} \mathrm{H}$ is more revealing than that of ${ }^{3} \mathrm{He}$. While there are no obvious outliers there is significant structure. The data points from Amroun seem to be low on average and the points from Collard appear to be high. The Beck data points seem fairly normally distributed. This means that if the normalization had been floated the $\chi^{2}$ could be reduced significantly as there do seem to be strong biases based on the experiments.

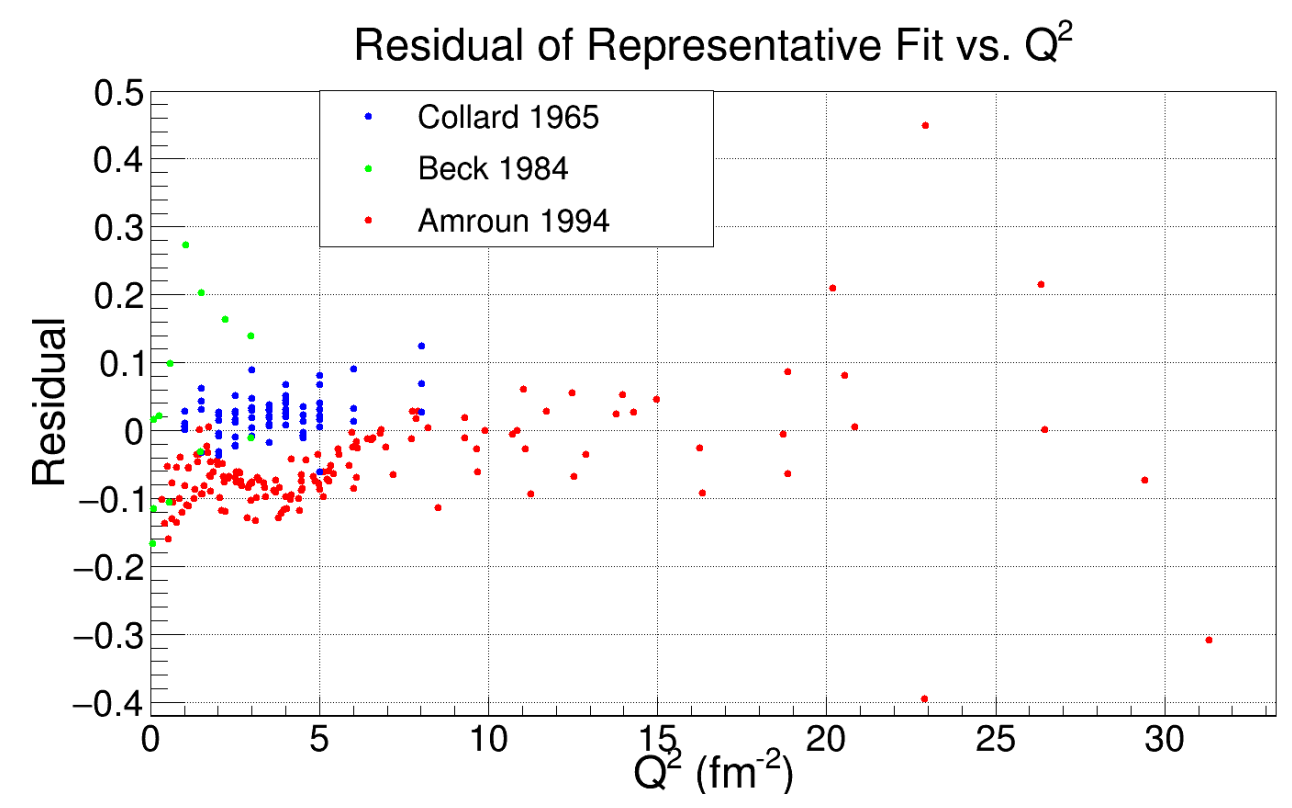

Figure 4.24: ${ }^{3}$ H Representative Fit Residual vs. $Q^{2}$.

\subsection{6 ${ }^{3}$ He Comparison with Theory and Previous Measurements}

Now that we have new form factor fits for ${ }^{3} \mathrm{He}$ and ${ }^{3} \mathrm{H}$ it is important to see how they compare to previous fits and theoretical predictions. We have already shown the previous fit from Amroun et al., but the error bands were previously excluded for clarity and will now be examined [29]. Four theory predictions from Marcucci et al. will also be compared to the current fits of the world data [56]. The four predictions include a 'conventional' approach, two $\chi$ EFT calculations, and finally a covariant spectator theorem prediction. These methods will be briefly described below, but for 
a more fulsome explanation of the theory predictions see the text in [56].

Paraphrasing the description of the 'conventional' approach used by Macucci et al. this technique simulates 2 and 3-body nucleon interactions within the nucleus and applies relativistic corrections. It also models the nucleon's interactions with external electroweak forces through both one and many-body currents. It removes nucleon resonances and replaces these with effective potentials and currents 56].

The $\chi$ EFT predictions in Marcucci et al. uses the chiral symmetry of quantum chromodynamics to describe the internal strong and EM interactions. This technique requires momentum space cutoffs to regularize operators with divergent behavior at large momenta. These cutoffs are selected at $500 \mathrm{MeV}$ for one model and $600 \mathrm{MeV}$ for the other $\chi$ EFT model [56].

The final theoretical model employed in Marcucci et al. is the covariant spectator theorem (CST). Again, paraphrasing [56], CST is a covariant field theory where nucleons and light mesons are used as the effective degrees of freedom. Currents and form factors can be extracted from the field theory's approximate solutions. This theory is also fully relativistic.

Figure 4.25 shows the new ${ }^{3} \mathrm{He} F_{c h}$ fits from this analysis, the representative fit from this analysis, Amroun et al.'s previous fit and error band, and the four theory predications from Marcucci et al. (Note that the error bands from Amroun and the theory curves from Marcucci were digitized with the tool found in [54]). It is immediately clear that the new fits (red) are in excellent agreement with the fit from Amroun et al. These fits are very tightly grouped and agree well until $Q^{2} \approx 60 \mathrm{fm}^{-2}$. This makes sense as the abundance of high precision low $Q^{2}$ data for ${ }^{3} \mathrm{He}$ means we expect the charge form factor to be well understood.

Examining how well theory is predicting the data fits we can see that the "conventional' approach (green) is doing a good job. This method locates the first minima well and also approximates the magnitude of $F_{c h}$ successfully. It is not totally in agreement with the data fits on the location of the second minimum, but so little data exists in this region that this difference hold little useful meaning. CST on the other hand, is 


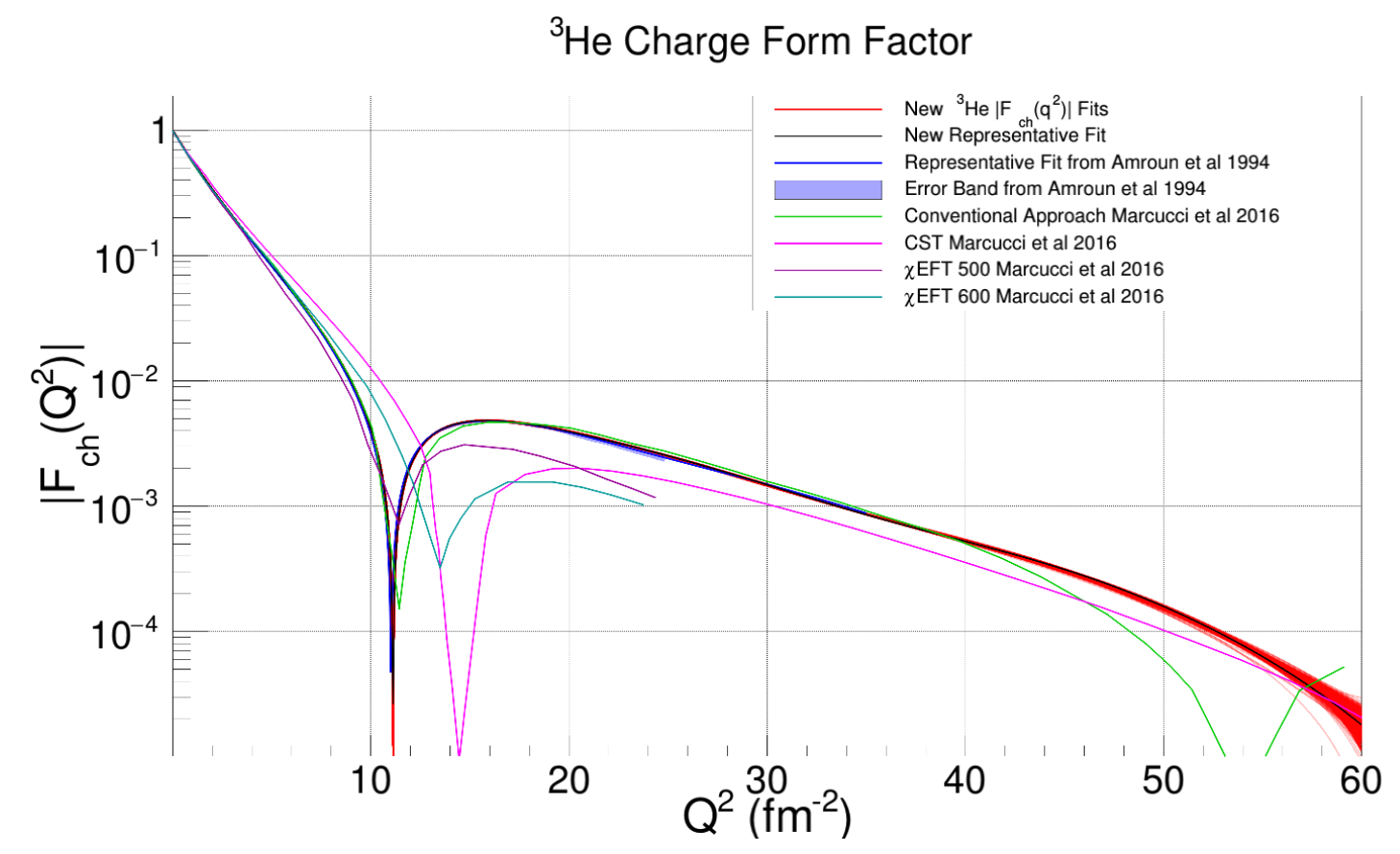

Figure 4.25: ${ }^{3} \mathbf{H e} \boldsymbol{F}_{\boldsymbol{c h}}$ Comparison of Results. The red lines are the individual fits from this analysis. The black line is the representative fit for this analysis. The blue line and shaded region is the fit from [29] and its error band. The theory predictions from [56] are the green, pink, purple, and blue lines for the 'conventional' approach, CST, $\chi \mathrm{EFT} 500$, and $\chi \mathrm{EFT} 600$ methods respectively.

doing a far worse job predicting the fits to data. CST estimates the first minimum significantly higher in $Q^{2}$ than the fits find, and CST also overestimates the magnitude of $F_{c h}$ below the first minimum and underestimates it above.

The $\chi$ EFT predictions are in disagreement with one another. The $\chi$ EFT prediction with a cutoff of $500 \mathrm{MeV}$ finds the first minimum successfully and then underestimates the magnitude of $F_{c h}$ above that minimum. Whereas, $\chi$ EFT 600 expects a minimum at higher $Q^{2}$ while also poorly predicting the magnitude of $F_{c h}$. Overall, the 'conventional' approach from [56] does the best job predicting the data fits and does so quite accurately.

Figure 4.26 mirrors Figure 4.25 but for the ${ }^{3} \mathrm{He}$ magnetic form factor. The new world data fits (red) are much less tightly grouped than they were for the ${ }^{3} \mathrm{He} F_{c h}$. This is because the $F_{m}$ is more dependent on the scarce high $Q^{2}$ and back angle data. Above $Q^{2}$ of $40 \mathrm{fm}^{-2}$ the fits diverge and actually split in to two distinct paths, one of 
which finds a second minimum before $Q^{2}=60 \mathrm{fm}^{-2}$, and the other which does not. When compared with the previous fits from [29] we see that the first minimum has shifted higher in $Q^{2}$ by approximately one to four $\mathrm{fm}^{-2}$. We also see that the $F_{m}$ magnitude of the new fits has decreased in magnitude above $Q^{2}$ of $25 \mathrm{fm}^{-2}$ with the addition of the new high $Q^{2}$ data.

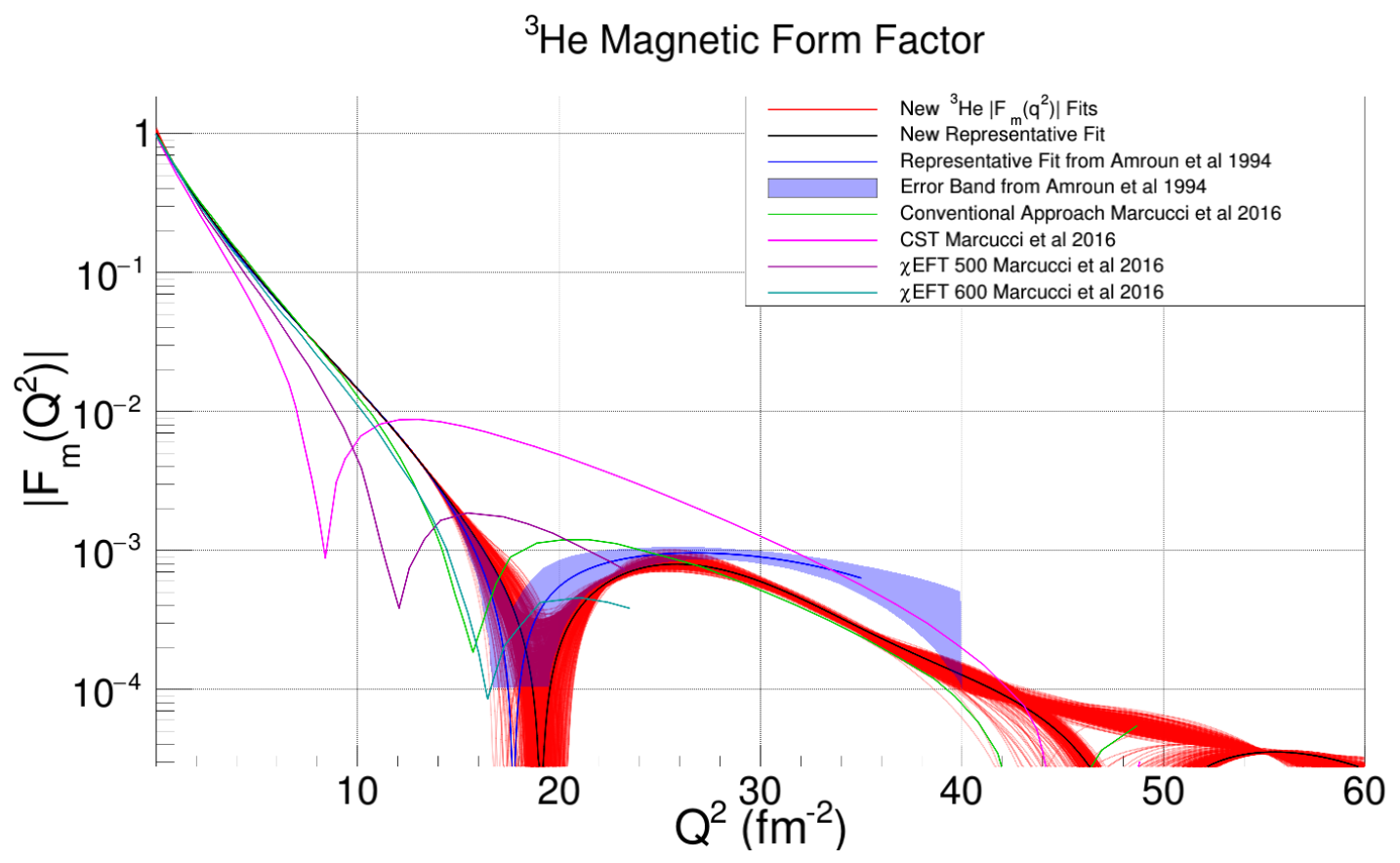

Figure 4.26: ${ }^{3} \mathbf{H e} \boldsymbol{F}_{\boldsymbol{m}}$ Comparison of Results. The red lines are the individual fits from this analysis. The black line is the representative fit for this analysis. The blue line and shaded region is the fit from [29] and its error band. The theory predictions from 56 are the green, pink, purple, and blue lines for the 'conventional' approach, CST, $\chi \mathrm{EFT} 500$, and $\chi \mathrm{EFT} 600$ methods respectively.

Turning once more to the theory predictions in [56, we see that all of the predictions predict a significantly lower $Q^{2}$ for the location of the first diffractive minimum. The 'conventional' approach and $\chi \mathrm{EFT} 600$ come closest to finding the location of the minimum but still fall short. The magnitude of $F_{m}$ estimated by the 'conventional' approach is too large in the region of the minimum; however, if the minimum of the 'conventional' approach were shifted up in $Q^{2}$ to match the world data fits it appears that the magnitude would then approximate $F_{m}$ relatively well. 
$\chi$ EFT 500 and the CST predictions both fail to predict the ${ }^{3} \mathrm{He} F_{m}$ well in either minimum location or magnitude. Overall, theory is struggling to predict the world data fits for $F_{m}$. It is notable that the new minimum location has actually shifted further away from theory and not closer to theory. This development merits further study to understand why the theory is poorly predicting the data.

This analysis found $\mathrm{a}^{3} \mathrm{He}$ charge radius of $1.90 \mathrm{fm}$ with a standard deviation of $0.00144 \mathrm{fm}$ from the new fits to world data in Section 4.3.3. Recall that there is an additional uncertainty on the charge radius due to not forcing the $\sum Q_{i}$ to equal unity as discussed in Section 4.3.3. Previous measurements from Saclay and Bates found charge radii of $1.96 \mathrm{fm} \pm 0.03 \mathrm{fm}$ and $1.87 \mathrm{fm} \pm 0.03 \mathrm{fm}$ respectively [55]. The new world data fits are slotting nicely in between these two experimental results, but the uncertainties do not quite overlap with the previous Saclay measurement. It is possible the uncertainties would overlap if this analysis had been able to quantify the additional uncertainty on the ${ }^{3} \mathrm{He}$ charge radius due to not fixing the $\sum Q_{i}$. Theoretical predictions of the ${ }^{3} \mathrm{He}$ charge radius also exist. Green's function Monte Carlo (GFMC) methods predict a charge radius of $1.96 \mathrm{fm} \pm 0.01 \mathrm{fm}$ and chiral effective field theory $(\chi \mathrm{EFT})$ predicts a radius of $1.962 \mathrm{fm} \pm 0.004 \mathrm{fm}[55]$. Both of these predictions are larger than the radius found by this analysis, but the experimental fit results and theory predictions are still fairly close.

Recall that the charge radius is determined by the slope of the charge form factor at a $Q^{2}$ of zero, and that the value of $F_{c h}$ at $Q^{2}=0$ (i.e. the $Y$-intercept of the charge form factor) is the same as the value of the $\sum Q_{i_{c h}}$. From physical considerations we expect $\sum Q_{i_{c h}}=1$ as discussed in Section 4.2. However, this analysis chose not to force the $Q_{i}$ parameters to sum to unity as not doing so provides another useful measure of the completeness of the world data without glossing over gaps and disagreements between the various measurements. For the ${ }^{3} \mathrm{He}$ fits the average $\sum Q_{i_{c h}}=1.00840$. The data and fit are doing an excellent job agreeing with our prior expectation that $\sum Q_{i_{c h}}=1$ with $\sum Q_{i_{c h}}$ only being slightly too high as seen in Figure 4.27. This slightly high $\sum Q_{i_{c h}}$ increases the negative magnitude of the $F_{c h}$ slope a small amount 
so the charge radius we find would have been very slightly smaller if we had forced $\sum Q_{i_{c h}}=1$.

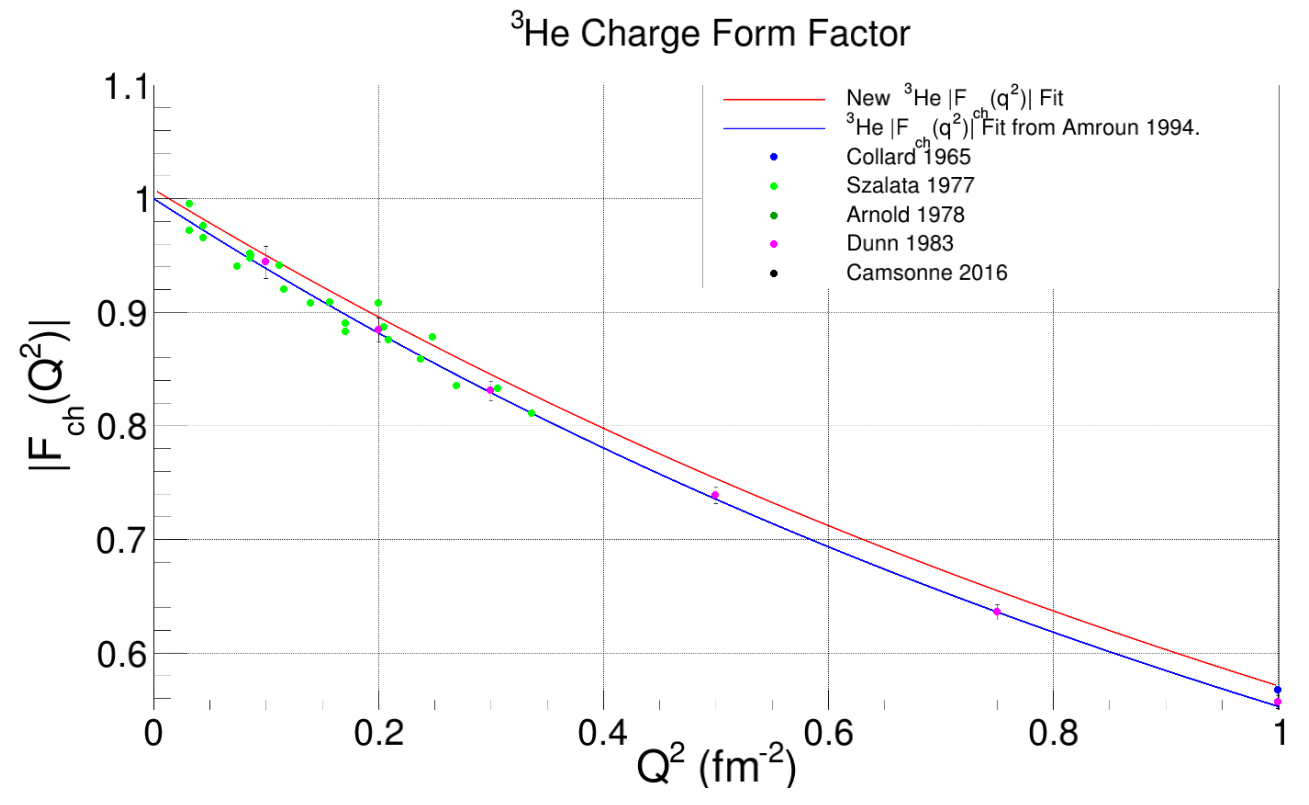

Figure 4.27: ${ }^{3} \mathbf{H e} \boldsymbol{F}_{\boldsymbol{c h}}$ at $\boldsymbol{Q}^{\mathbf{2}}=\mathbf{0}$. This plot shows the ${ }^{3}$ He charge form factor near $Q^{2}=0$. The red line of the new form factor fit clearly has a $Y$-intercept of slightly greater than unity unlike the older blue line fit from [29]. This different intercept slightly changes the charge radius extracted for ${ }^{3} \mathrm{He}$ by altering the slope at $Q^{2}=0$.

\subsection{7 $\quad{ }^{3} \mathrm{H}$ Comparison with Theory and Previous Measurements}

We can apply the same comparisons to past fits and theory predictions performed in Section 4.3 .6 to ${ }^{3} \mathrm{H}$ as well. Figure 4.28 shows the charge form factor fits and theory predictions for ${ }^{3} \mathrm{H}$ in the same manner as was done for ${ }^{3} \mathrm{He}$. (Note that the error bands from Amroun and the theory curves from Marcucci were digitized with the tool found in [54]). Comparing this analysis' fits to [29 they are in good agreement. The first minimum is almost identical, and the error bands overlap nicely, although there is a slight difference in magnitude after the first minimum. This is likely due to this analysis having access to the data of fewer experiments than 29 had. The fits diverge above $Q^{2}$ of $25 \mathrm{fm}^{-2}$ due to a lack of data in this region. Since no new elastic ${ }^{3} \mathrm{H}$ data has been added to the world data we expect to have strong agreement with the 
previous fits of the same data, and the agreement of the two results demonstrates the consistency of our technique with past techniques.

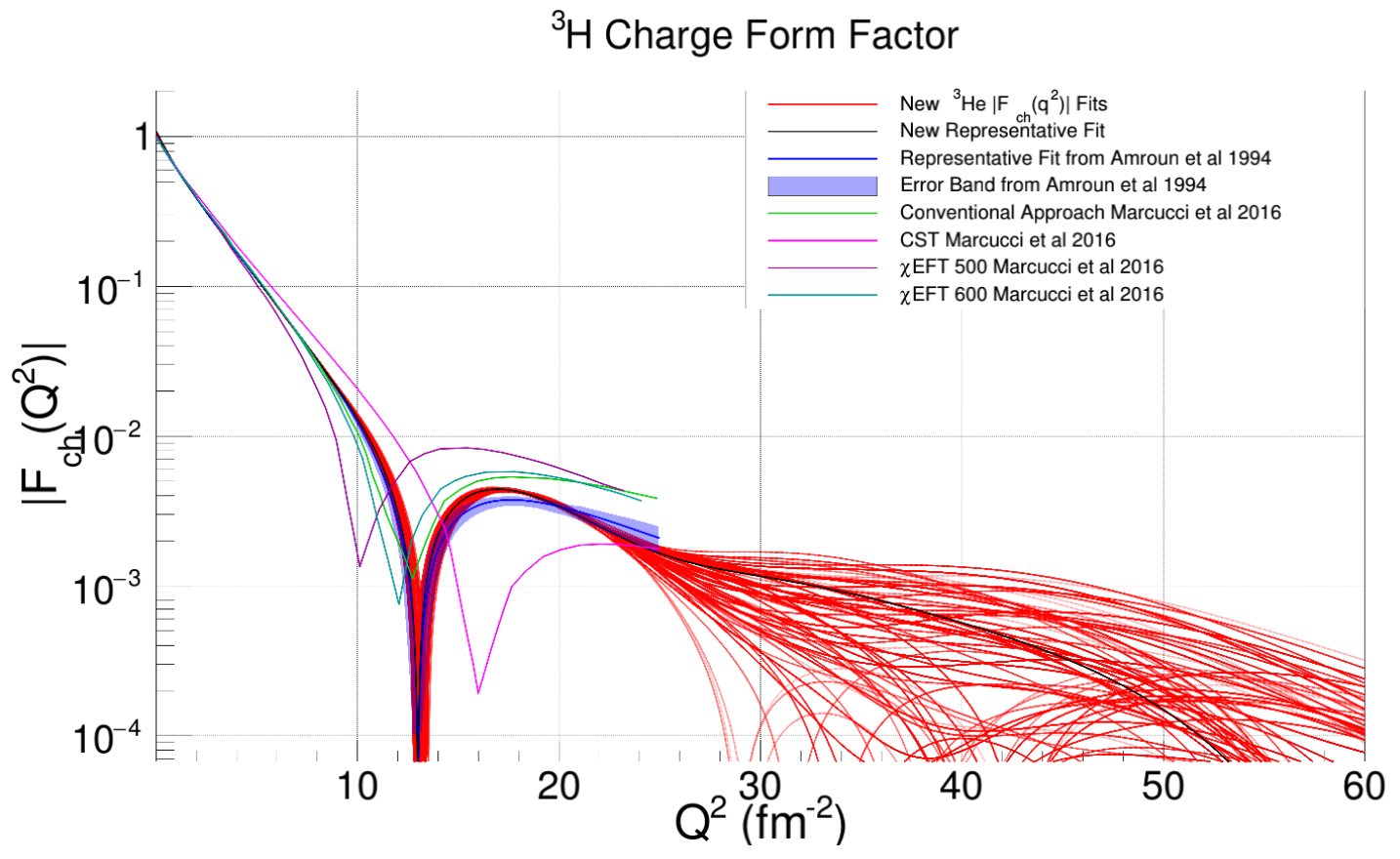

Figure 4.28: ${ }^{3} \mathbf{H} \boldsymbol{F}_{\boldsymbol{c h}}$ Comparison of Results. The red lines are the individual fits from this analysis. The black line is the representative fit for this analysis. The blue line and shaded region is the fit from [29] and its error band. The theory predictions from [56] are the green, pink, purple, and blue lines for the 'conventional' approach, CST, $\chi \mathrm{EFT} 500$, and $\chi \mathrm{EFT} 600$ methods respectively.

It should be noted that JLab has recently gathered new ${ }^{3} \mathrm{H}$ elastic data that has yet to be analyzed. Integrating this new data with this analysis should be a simple matter after the data are analyzed. However, the new data are not at very high $Q^{2}$ so it may not influence the fits greatly. The new data are useful in that they overlap with data from [36] and can be used to normalize that data such that it can be incorporated in future fits.

Turning to the theory predictions, the 'conventional' approach is performing best once more. It is successfully finding the first minimum, but it predicts a significantly larger $F_{c h}$ magnitude beyond the minimum. $\chi$ EFT 600 similarly predicts the minimum while also overestimating the magnitude of the form factor. $\chi$ EFT 500 underestimates 
the location of the minimum in $Q^{2}$ while overestimating the magnitude by an even greater value. Finally, CST greatly overestimates the $Q^{2}$ location of the first minimum while also underestimating the form factor magnitude. The 'conventional' and $\chi \mathrm{EFT}$ 600 models predicted the minimum well, but it appears that there is still a need to better understand the magnitude of ${ }^{3} \mathrm{H}$ 's $F_{c h}$.

Figure 4.29 shows the magnetic form factor fits and theory predictions for ${ }^{3} \mathrm{H}$ in the same manner as was done previously. Like the charge form factor, the magnetic form factor updated world data fits are in strong agreement with the previous fit in [29]. The $F_{m}$ error band comprised by the new fits almost perfectly overlaps with Amroun et al. Again, no new data was added, so this is to be expected. The fits are grouped relatively well until $Q^{2}$ of $30 \mathrm{fm}^{-2}$ after which they begin to diverge from each other.

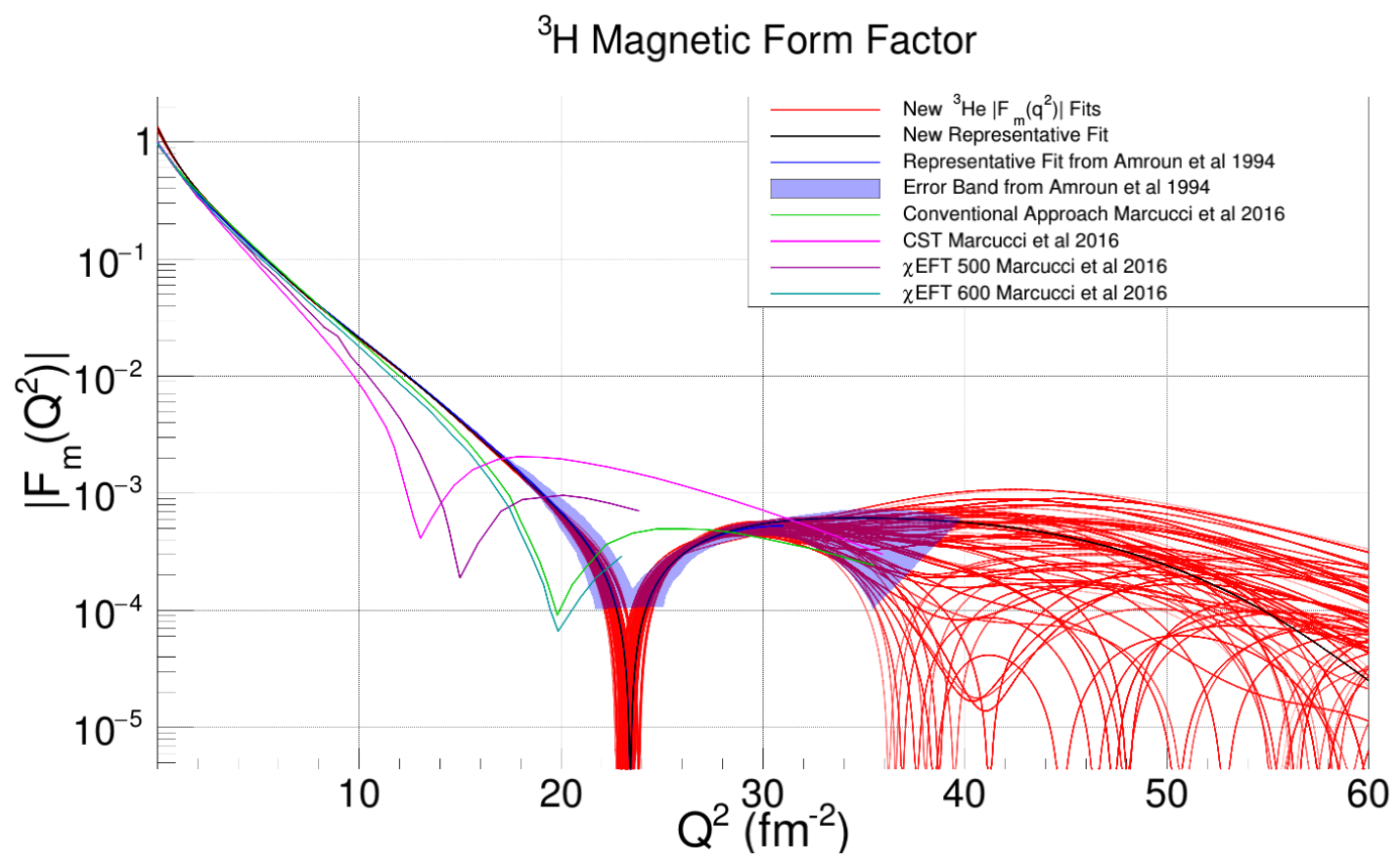

Figure 4.29: ${ }^{3} \mathbf{H} \boldsymbol{F}_{\boldsymbol{m}}$ Comparison of Results. The red lines are the individual fits from this analysis. The black line is the representative fit for this analysis. The blue line and shaded region is the fit from [29] and its error band. The theory predictions from [56] are the green, pink, purple, and blue lines for the 'conventional' approach, CST, $\chi \mathrm{EFT} 500$, and $\chi \mathrm{EFT} 600$ methods respectively.

Theory predictions for the magnetic form factor of ${ }^{3} \mathrm{H}$ all anticipate a significantly 
lower $Q^{2}$ first minimum than is indicated by fits of the world data. The 'conventional' approach and $\chi$ EFT 600 come closest to finding the minimum but still underestimate it by three or four $\mathrm{fm}^{-2}$. These two predictions also overestimate the magnitude of $F_{m}$, but if they were shifted up in $Q^{2}$ to match the location of the first minimum the magnitudes would line up with the new fits decently. The $\chi$ EFT 500 and CST fits both predict a first minimum that is far too low while also overestimating the $F_{m}$ magnitude.

Examining the success of the four theoretical models at predicting the experimental data it is clear that the 'conventional' approach, modelling two and three-body interactions with relativistic corrections, was the most successful. $\chi$ EFT predictions often came close to matching the 'conventional' model's success, but the $\chi$ EFT models were heavily dependent on their momentum space cutoffs. The CST model generally did a poor job at predicting the data. The successful theories are doing a good job predicting the charge form factor, $F_{c h}$, although they miss the magnitude a bit in ${ }^{3} \mathrm{H}$. However, all of the models seem to be having a more difficult time predicting the magnetic form factor both in minima location and magnitude.

This analysis found $\mathrm{a}^{3} \mathrm{H}$ charge radius of $2.02 \mathrm{fm}$ with a standard deviation of $0.0133 \mathrm{fm}$ as discussed in Section 4.3.5. Recall that there is an additional uncertainty on the charge radius due to not forcing the $\sum Q_{i}$ to equal unity as discussed in Section 4.3.3, and this additional uncertainty is likely to be significant as discussed in Section 4.3.5. Past measurements from Saclay found a charge radius of $1.76 \mathrm{fm} \pm 0.09 \mathrm{fm}$, and measurements from Bates found a charge radius of $1.68 \mathrm{fm} \pm 0.03 \mathrm{fm}$ [55]. GFMC predictions estimate a radius of $1.77 \mathrm{fm} \pm 0.01 \mathrm{fm}$, and $\chi \mathrm{EFT}$ predicts a radius of $1.756 \mathrm{fm} \pm 0.006 \mathrm{fm}$.

We also discussed in Section 1.3 how we expect the ${ }^{3} \mathrm{He}$ charge radius (found to be $1.90 \mathrm{fm}$ in this analysis) to be larger than the ${ }^{3} \mathrm{H}$ charge radius which does not appear to be the case here. Clearly the new world data fits are finding a significantly larger charge radius than past results and theory. However, it is easy to see why this analysis is in disagreement with past measurements. Because this analysis did not force $\sum Q_{i_{c h}}$ 
$=1$ as discussed in Sections 4.2 and 4.3.6, the slope of the form factor at $Q^{2}=0$ is purely determined by the free parameters $Q_{i_{c h}}$. Once the additional uncertainty on the charge radius from not forcing the $\sum Q_{i_{c h}}=1$ is accounted for the results may be in much more reasonable agreement. Unfortunately, this analysis was unable to quantify this additional uncertainty.

Examining this more closely, the ${ }^{3} \mathrm{H}$ charge radius has an average $\sum Q_{i_{c h}}$ for the new world data fits of 1.08991, or almost $9 \%$ larger than if the free parameters were forced to sum to unity. This higher $F_{c h}$ value at $Q^{2}=0$, shown in Figure 4.30 , means that the negative slope of the form factor has a larger magnitude than if $F_{c h}$ were forced to unity at $Q^{2}=0$. Equation 1.19 shows that a larger negative slope will yield a larger charge radius. This analysis' larger radius is due to not forcing $\sum Q_{i_{c h}}=1$ as past fits have done. (The $\sum Q_{i_{m}}$ significantly diverging from unity is why magnetic radii were not calculated in this analysis since their values would be dominated by not forcing the $\sum Q_{i_{m}}$ to unity.) By not forcing $\sum Q_{i_{c h}}=1$ we can see that the 1984 Beck data 37] is pulling the charge form factor up at very low $Q^{2}$. Now it is clear that the ${ }^{3} \mathrm{H}$ world data is either less complete and accurate and/or there is more disagreement between the different experiments' results (in this case it is both) than is the case for

${ }^{3} \mathrm{He}$. This would have been hard to identify if the $\sum Q_{i_{c h}}$ were forced to sum to unity or if we had floated the normalizations of the different experiments.

\subsection{8 ${ }^{3} \mathrm{He}$ and ${ }^{3} \mathrm{H}$ Comparison}

Now that we have found new representative form factors for both ${ }^{3} \mathrm{He}$ and ${ }^{3} \mathrm{H}$, we can return to the fact that these are mirror nuclei and compare their form factors and charge densities. Recall that the only differences in these quantities will be due to the different Coulomb forces in the nuclei since the strong force does not distinguish between protons and neutrons as discussed in Section 1.4 .

Figure 4.31 shows the ${ }^{3} \mathrm{He}$ and ${ }^{3} \mathrm{H}$ charge form factors plotted together. Notice that the first diffractive minimum for ${ }^{3} \mathrm{He}$ comes before the first ${ }^{3} \mathrm{H}$ minimum. As discussed in Section 1.4 this indicates that ${ }^{3} \mathrm{He}$ has a larger charge radius than ${ }^{3} \mathrm{H}$. This 


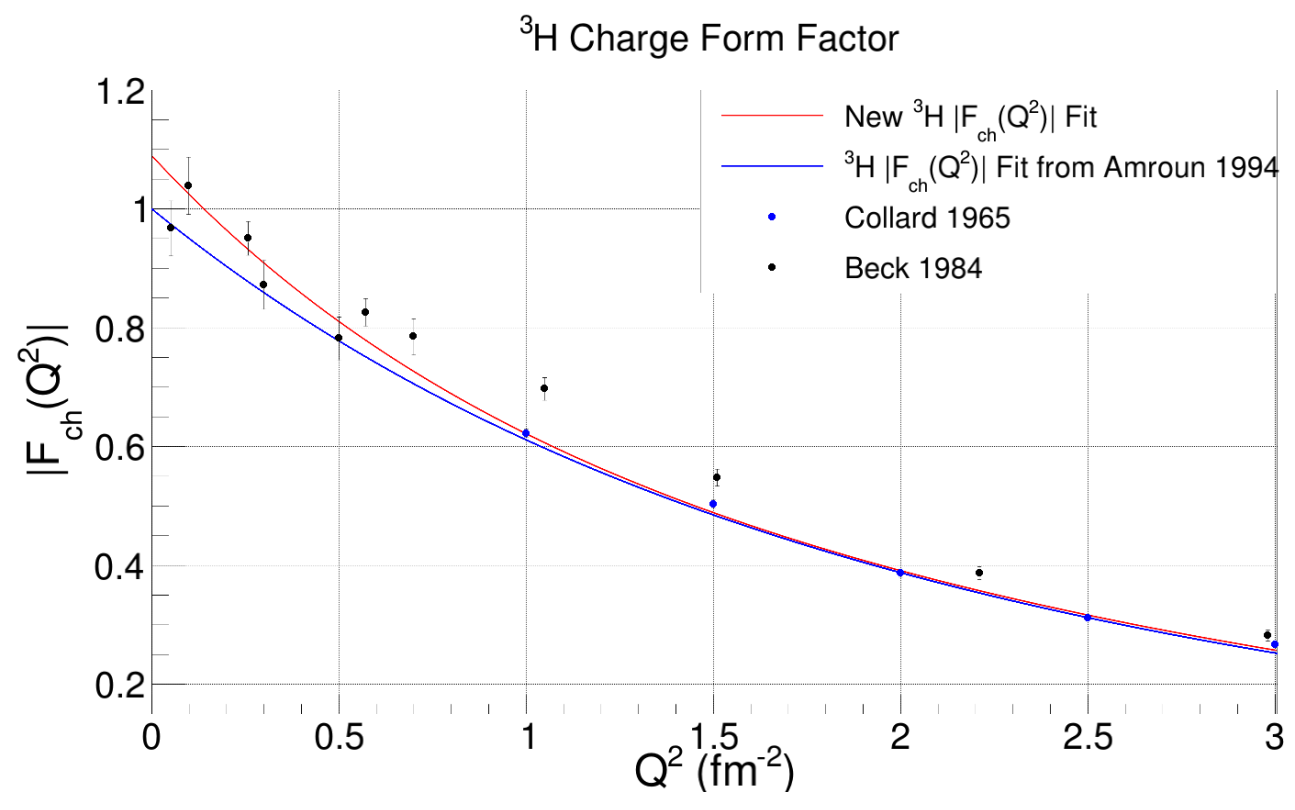

Figure 4.30: ${ }^{3} \mathbf{H} \boldsymbol{F}_{\boldsymbol{c h}}$ at $\boldsymbol{Q}^{\mathbf{2}}=\mathbf{0}$. This plot shows the ${ }^{3} \mathrm{H}$ charge form factor near $Q^{2}$ $=0$. The red line of the new form factor fit has a $Y$-intercept $\approx 9 \%$ greater than unity unlike the older blue line fit from [29]. This different intercept significantly changes the charge radius extracted for ${ }^{3} \mathrm{H}$ by altering the slope at $Q^{2}=0$.

is because ${ }^{3} \mathrm{He}$ has a stronger repulsive Coulomb force than ${ }^{3} \mathrm{H}$ which pushes the radius further out against the binding strong force. We can use Equation 1.12 to estimate what the charge radii would be if these nuclei could be modelled as homogeneous hard spheres of charge. The first minimum for ${ }^{3} \mathrm{He}$ is located at $Q^{2} \approx 11 \mathrm{fm}^{-2}$ which estimates a charge radius of $\frac{4.5}{\sqrt{11 \mathrm{fm}^{-2}}}=1.36 \mathrm{fm}$. Whereas, the first minimum for ${ }^{3} \mathrm{H}$ is located at $Q^{2} \approx 13 \mathrm{fm}^{-2}$ which estimates a charge radius of $\frac{4.5}{\sqrt{13 \mathrm{fm}^{-2}}}=1.25 \mathrm{fm}$. The new fits found a ${ }^{3} \mathrm{He}$ charge radius of $1.90 \mathrm{fm}$, and a ${ }^{3} \mathrm{H}$ charge radius of 2.02 $\mathrm{fm}$ (remember there is significant uncertainty on the ${ }^{3} \mathrm{H}$ charge radius as discussed in 4.3.5). These larger radii show that these nuclei are not simply hard spheres of charge. In reality the charge densities fall off more gradually with the radius as seen in Figure 4.33 .

We also see that the new fits find a larger charge radius for ${ }^{3} \mathrm{H}$ than ${ }^{3} \mathrm{He}$. This is in spite of the fact that ${ }^{3} \mathrm{H}$ has a later first diffractive minimum than ${ }^{3} \mathrm{He}$ indicating that the ${ }^{3} \mathrm{H}$ charge radius should be smaller. We know that the ${ }^{3} \mathrm{H}$ charge radius 


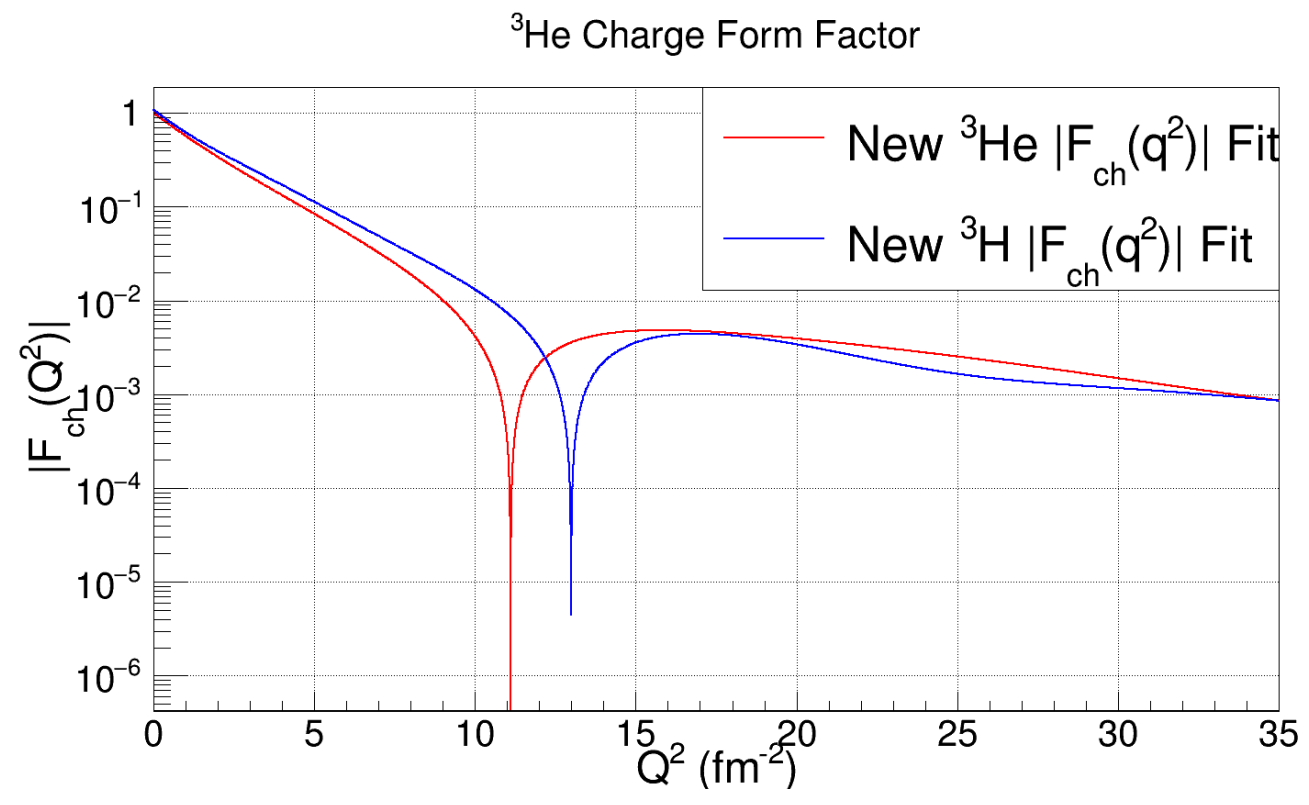

Figure 4.31: $\boldsymbol{F}_{\boldsymbol{c h}}$ Comparison This plot shows the new representative charge form factor fits for ${ }^{3} \mathrm{He}$ (red line) and ${ }^{3} \mathrm{H}$ (blue line). $F_{c h}$ is only plotted up to $Q^{2}=35$ $\mathrm{fm}^{-2}$ since beyond this range the individual fits begin to diverge.

looks artificially large due to not forcing the $\sum Q_{i_{c h}}=1$ as discussed in Section 4.3.7. This is because the $\sum Q_{i_{c h}}$ changes the slope of the form factor near $Q^{2}=0$, but the location of the first diffractive minima are barely affected by the $\sum Q_{i_{c h}}$. This means that the relative locations of the first ${ }^{3} \mathrm{He}$ and ${ }^{3} \mathrm{H}$ minima still accurately reflect the relative sizes of the charge radii (i.e. that the ${ }^{3} \mathrm{He}$ charge radius is larger than the ${ }^{3} \mathrm{H}$ charge radius). This same comparison is also shown for the magnetic form factors in Figure 4.32. While this analysis did not study magnetic radii, Figure 4.32 shows that the ${ }^{3} \mathrm{He}$ magnetic radius should be larger than that of the ${ }^{3} \mathrm{H}$ magnetic radius.

Lastly, we can compare the charge densities of ${ }^{3} \mathrm{He}$ and ${ }^{3} \mathrm{H}$ as in Figure 4.33 . We see that there is more total electric charge for ${ }^{3} \mathrm{He}$ than ${ }^{3} \mathrm{H}$. This is clearly due to ${ }^{3} \mathrm{He}$ having two protons and ${ }^{3} \mathrm{H}$ only having one. Integrating over the total charges for each nuclei one finds that ${ }^{3} \mathrm{He}$ has a total charge of $2.01 e$, and ${ }^{3} \mathrm{H}$ has a total charge of $1.09 e$ with $e$ being the elementary charge. These values are slightly higher than we expect from the atomic numbers, but this is again a consequence of not forcing the $\sum Q_{i_{c h}}$ to unity. We also see that the charge density of ${ }^{3} \mathrm{He}$ extends out beyond that 


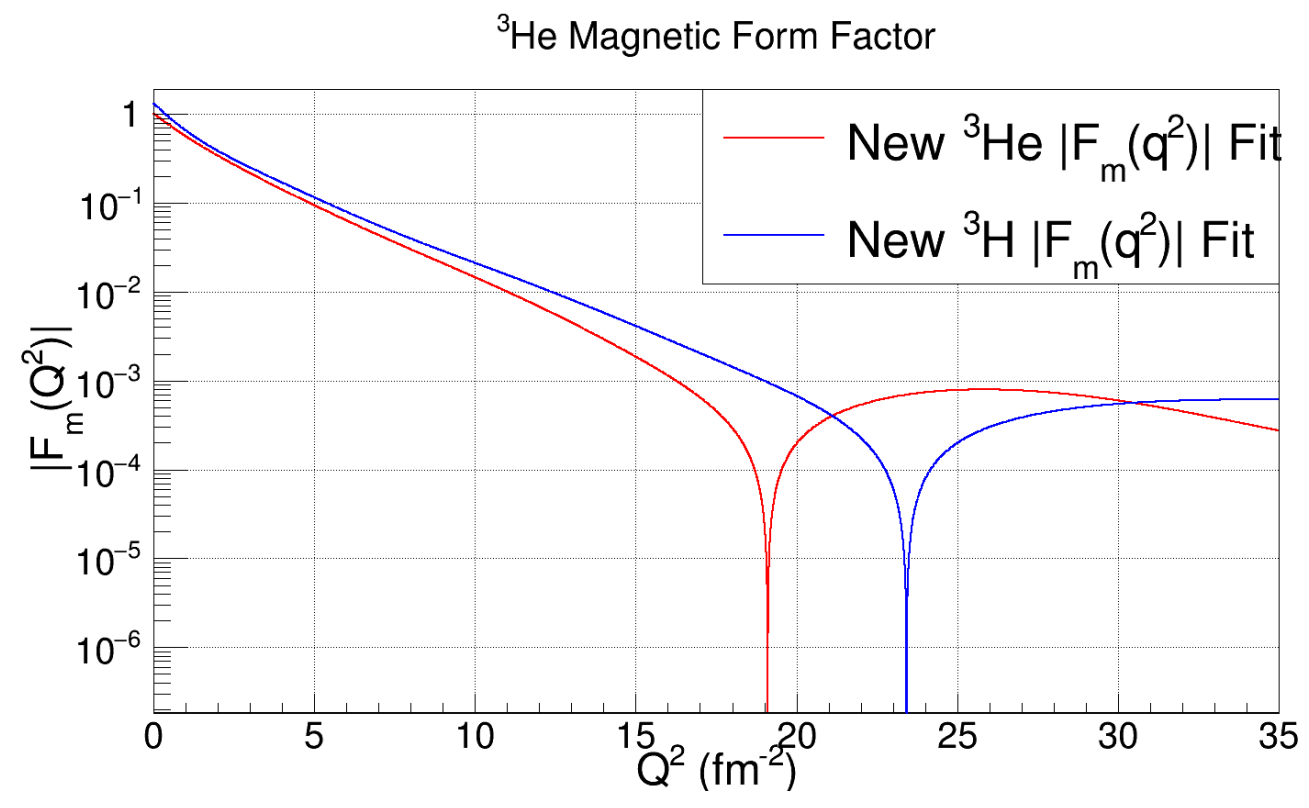

Figure 4.32: $\boldsymbol{F}_{\boldsymbol{m}}$ Comparison This plot shows the new representative magnetic form factor fits for ${ }^{3} \mathrm{He}$ (red line) and ${ }^{3} \mathrm{H}$ (blue line). $F_{c h}$ is only plotted up to $Q^{2}=35$ $\mathrm{fm}^{-2}$ since beyond this range the individual fits begin to diverge.

of ${ }^{3} \mathrm{H}$.

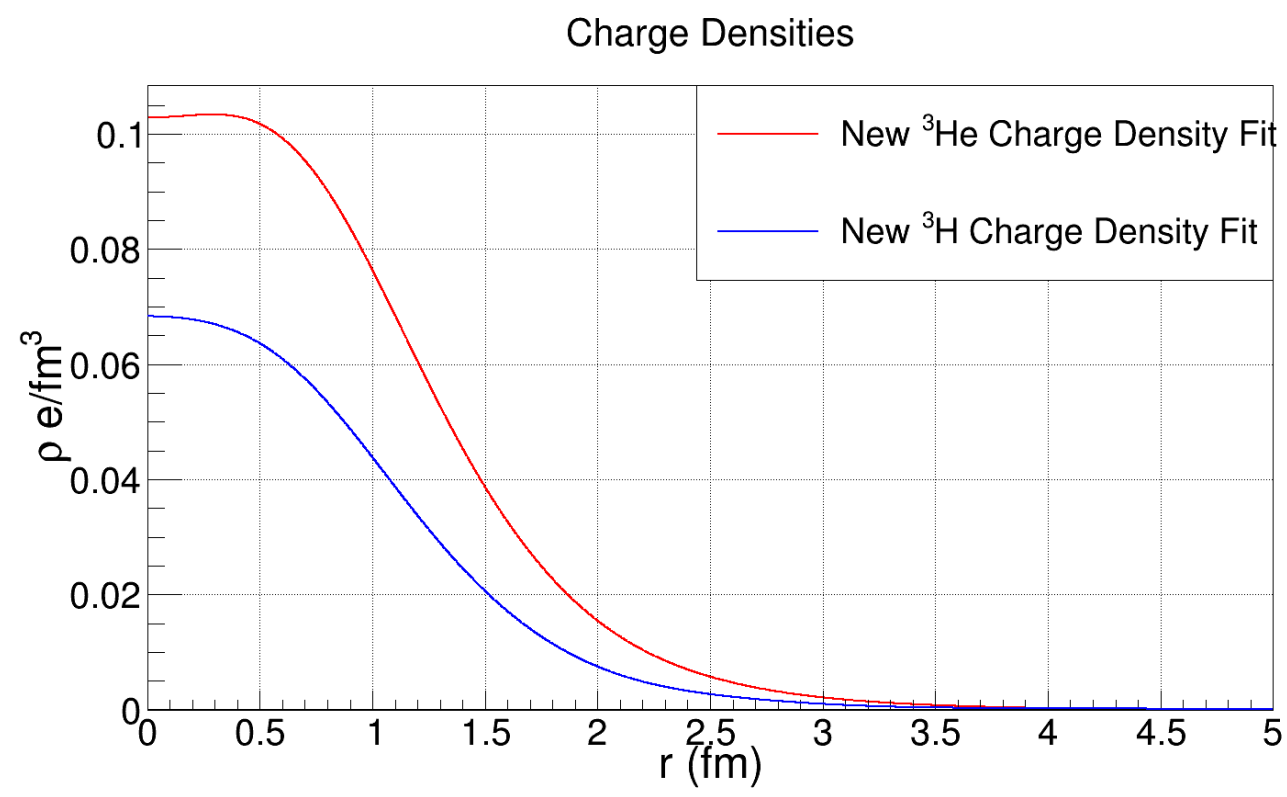

Figure 4.33: Charge Density Comparison This plot shows the new representative charge density fits for ${ }^{3} \mathrm{He}$ (red line) and ${ }^{3} \mathrm{H}$ (blue line). The units of the $Y$-axis are the elementary electron charge per cubic fermi. 
The ${ }^{3} \mathrm{He}$ plateau region near $Q^{2}=0$ is wider than the corresponding plateau in ${ }^{3} \mathrm{H}$ indicating that there is a larger region of near constant charge density at the center of the ${ }^{3} \mathrm{He}$ nucleus than the ${ }^{3} \mathrm{H}$ nucleus. (However, there is more uncertainty on the ${ }^{3} \mathrm{H}$ charge density near $Q^{2}=0$ as seen in Figure $4.16 \mathrm{~b}$ which means a range of plateau shapes are possible for ${ }^{3} \mathrm{H}$ ). While it is clear that the different Coulomb forces in the two nuclei cause some differences, the total shapes of the form factors and charge densities are quite similar due to the mirror nuclei having the same ground state shell-model states. 


\section{Chapter 5}

\section{Conclusions}

A new ${ }^{3} \mathrm{He}$ elastic cross section was extracted from JLab Hall A experiment E08014. This required isolating the elastically scattered electrons from a large quasielastic background. This analysis found an elastic electron yield of 627 electrons. The small number of elastic events contributed strongly to the uncertainty in this new cross section. The elastic electron yield was simulated using the Monte Carlo SIMC which contained an older model of elastic ${ }^{3} \mathrm{He}$ form factors from 29 . The elastic SIMC results were then summed with a fit of the quasieastic background of the experimental data so as to be comparable to the full experimental yield (elastic and quasielastic events). The SIMC yield was then scaled to match the experimental yield so that SIMC would produce the experimental cross section. Bin centering corrections were then applied to find the $Q^{2}$ at which to place the new cross section, and finally the various sources of uncertainty were compiled. This resulted in a new ${ }^{3} \mathrm{He}$ elastic cross section measurement of $1.335 \times 10^{-6} \mu \mathrm{b} / \mathrm{sr} \pm 0.086 \times 10^{-6} \mu \mathrm{b} / \mathrm{sr}$ at a $Q^{2}$ value of $34.19 \mathrm{fm}^{-2}$.

The world data for both ${ }^{3} \mathrm{H}$ and ${ }^{3} \mathrm{He}$ elastic cross sections were then collected with the addition of new JLab high $Q^{2}$ data and this analysis' cross section. These cross sections were then fit with a sum of Gaussians parametrization which allowed the charge and magnetic form factors to be extracted. Representative charge and magnetic form factor parametrizations were chosen from the center of each set of new SOG form 
factor fits for ${ }^{3} \mathrm{H}$ and ${ }^{3} \mathrm{He}$. Error bands were then defined about these representative fits spanning the width of all possible 'good' SOG fits. Figure 4.20 shows the new representative form factor fits for ${ }^{3} \mathrm{H}$ and the fit parameters are given in Table 4.9 . Figure 4.8 shows the new representative form factor fits for ${ }^{3} \mathrm{He}$ and the fit parameters are given in Table 4.6 .

The charge and magnetic form factors for ${ }^{3} \mathrm{H}$ remained consistent with past fits from 29 since no new data has been added to the world data yet. The charge form factor for ${ }^{3} \mathrm{He}$ also remained unchanged with past fits since there is an abundance of excellent data influencing $F_{c h}$. The magnetic form factor for ${ }^{3} \mathrm{He}$ did change significantly with the addition of new high $Q^{2}$ data. The first diffractive minimum in $F_{m}$ shifted higher in $Q^{2}$ by several $\mathrm{fm}^{-2}$, and the magnitude of $F_{m}$ decreased somewhat after the first minimum. Overall, our knowledge of the form factors declines considerably at higher $Q^{2}\left(<\approx 25-30 \mathrm{fm}^{-2}\right)$.

The new fits were compared to past fits of the data from Amroun et al. 29] and theory predictions from Marcucci et al. 56. The ${ }^{3} \mathrm{H}$ charge form factor fits and theory predictions can be found in Figure 4.28 , and the ${ }^{3} \mathrm{H}$ magnetic form factor fits and theory predictions can be found in Figure 4.29. The ${ }^{3} \mathrm{He}$ charge form factor fits and theory predictions can be found in Figure 4.25, and the ${ }^{3} \mathrm{He}$ magnetic form factor fits and theory predictions can be found in Figure 4.26. The new form factor fits broadly agreed with the past Amroun fits except for the shift in the ${ }^{3} \mathrm{He}$ magnetic form factor discussed above. Theory is doing a good job predicting the charge form factors using a 'conventional' approach which accounts for two and three-body nucleon interactions and relativistic corrections. $\chi$ EFT predictions are also often successful at predicting the charge form factors. However, theory struggles to predict the magnetic form factors of either ${ }^{3} \mathrm{H}$ or ${ }^{3} \mathrm{He}$.

Charge densities were then calculated along with charge radii for both targets using the new form factor fits. The average charge radius for ${ }^{3} \mathrm{He}$ was found to be $1.90 \mathrm{fm}$ with a standard deviation of $0.00144 \mathrm{fm}$. This value is in decent agreement with past measurements (Saclay $1.96 \mathrm{fm} \pm 0.03 \mathrm{fm}$ and Bates $1.87 \mathrm{fm} \pm 0.03 \mathrm{fm}$ ). However, 
the average charge radius for ${ }^{3} \mathrm{H}$ was found to be $2.02 \mathrm{fm}$ with a standard deviation of $0.0133 \mathrm{fm}$. This value is much larger than past measurements (Saclay $1.76 \mathrm{fm} \pm$ $0.09 \mathrm{fm}$ and Bates $1.68 \mathrm{fm} \pm 0.03 \mathrm{fm})$. Recall that there is an additional uncertainty on each of the charge radius results due to not forcing the $\sum Q_{i}$ to equal unity as discussed in Section 4.3.3, and this additional uncertainty is much larger for ${ }^{3} \mathrm{H}$. Not restricting the free parameters in this manner caused the magnitude of the negative slope of the ${ }^{3} \mathrm{H} F_{c h}$ at a $Q^{2}$ of zero to increase causing a larger radius to be found, but this choice also gives us another method to evaluate how well the new fits comply with our physical expectation that $\sum Q_{i_{c h}}=1$ as discussed in Section 4.2. Unfortunately, this analysis was unable to quantify the additional uncertainty on the charge radii due to not forcing the $\sum Q_{i_{c h}}=1$, but if this uncertainty were accounted for the ${ }^{3} \mathrm{H}$ results in particular would likely be in much better agreement with past measurements.

The updated representative form factors were then used to replace the older parametrization of the form factors in SIMC. Using the updated SIMC Monte Carlo the ${ }^{3} \mathrm{He}$ elastic cross section from experiment E08-014 was recalculated using the elastic electron yield from the modified SIMC code. These two cross section values, the newer and older parametrizations, should be in agreement with one another using the cross section extraction technique discussed in Chapter 3. In fact, the updated form factor SIMC model finds a cross section of $1.345 \times 10^{-6} \mu \mathrm{b} / \mathrm{sr} \pm 0.086 \times 10^{-6} \mu \mathrm{b} / \mathrm{sr}$ at a $Q^{2}$ value of $34.19 \mathrm{fm}^{-2}$ as opposed to the older model which estimated $1.335 \times 10^{-6}$ $\mu \mathrm{b} / \mathrm{sr}$.

Going forward, there are several logical extensions to this work. It should be relatively straightforward to expand the fitting code to fit the cross sections of other light nuclei. If the code were to be expanded to heavier nuclei a full phase shift correction would need to be applied in place of $Q_{e f f}^{2}$. Performing these fits with different functions like Fourier-Bessel functions would make for an interesting point of comparison to the SOG fits. Collecting more high $Q^{2}$ and back angle data would considerably improve our understanding of the form factors, especially $F_{m}$. JLab's Hall $\mathrm{A}$ is well equipped to make these measurements with its maximum back angle of 
$150^{\circ}$ and maximum beam energy of $11 \mathrm{GeV}$.

The discrepancy between theory and experiment on the location of the first diffractive minimum for the ${ }^{3} \mathrm{He} F_{m}$ could also be resolved by JLab. By performing an asymmetry measurement using a polarized ${ }^{3} \mathrm{He}$ target and a polarized electron beam the location of the $F_{m}$ minimum can be found. This is because the asymmetry will flip sign when passing the diffractive minimum. This asymmetry measurement is given in Equation 5.1, where $N^{+}$is the normalized counting rate for positive beam helicity and $N^{-}$is the normalized counting rate for negative beam helicity 57. This experiment would determine whether theory is wrong, experiment is wrong, or both are wrong on the location of the ${ }^{3} \mathrm{He}$ magnetic form factor's first diffractive minimum.

$$
A_{\text {meas }}=\frac{N^{+}-N^{-}}{N^{+}+N^{-}}
$$




\section{References}

[1] B. Povh et al., Particles and Nuclei an Introduction to the Physical Concepts (Springer, Reading, Mass, 2008).

[2] L. N. Hand, D. G. Miller, and R. Wilson, "Electric and Magnetic Form Factors of the Nucleon", Reviews of Modern Physics 35, 335 (1963).

[3] A. Camsonne et al., "JLab Measurements of the ${ }^{3} \mathrm{He}$ Form Factors at Large Momentum Transfers", Physical Review Letters 119, 162501 (2017).

[4] W. Leo, Introductory Nuclear Physics (John Wiley \& Sons, 1987).

[5] W. von Schlippe, Selected Topics in Physics, http://www.phys . spbu .ru/ content/File/Library/studentlectures/schlippe/cm07-11.pdf

[6] A. Alcorn et al., "Basic instrumentation for Hall A at Jefferson Lab", Nuclear Instruments and Methods in Physics Research A, 294 (2003).

[7] Z. Ye, "Short Range Correlations in Nuclei at Large $x_{B j}$ through Inclusive QuasiElastic Electron Scattering", Ph.D. Thesis, https://userweb.jlab.org/ yez/ thesis/ZYe_Thesis.pdf, (University of Virginia, Dec. 2013).

[8] P. Solvignon and Z. Ye, $X>2$ experiment (E08-014), https://hallaweb.jlab org/experiment/E08-014/.

[9] C. Leeman, D. Douglas, and G. Krafft, "THE CONTINUOUS ELECTRON BEAM ACCELERATOR FACILITY: CEBAF at the Jefferson Laboratory", Annual Review of Nuclear and Particle Science 51, 413 (2001),

[10] Y. Wang, "Measurement of the Proton Elastic Cross Section at $Q^{2}=0.66$, 1.10, 1.51 and 1.65 GeV" ", Ph.D. Thesis, https://wm1693.app.box.com/s/ dsya4olvfo891h73t415/file/192300626229, (The College of William \& Mary, Aug. 2017).

[11] W. Barry et al., Beam Position Measurement in the CEBAF Recirculating Linacs by Use of Pseudorandom Pulse Sequences, tech. rep. JLab-TN-90-246 (Southeastern Universities Research Association, 1990).

[12] W. Barry et al., Basic Noise Considerations for CEBAF Beam Position Monitors, tech. rep. JLab-TN-91-087 (Southeastern Universities Research Association, 1991).

[13] K. Unser, "A Toroidal DC Beam Current Transformer with High Resolution", IEEE Transactions on Nuclear Science NS-28, 2344 (1981).

[14] P. Solvignon, "BCM Calibration for E08-014", https://userweb.jlab.org/ _solvigno/src/e08014_analysis/xgt2_bcm_calib.pdf, 2012. 
[15] K. Fissum et al., "Vertical Drift Chambers for the Hall A High-resolution Spectrometers at Jefferson Lab", Nuclear Instruments and Methods in Physics Research A, 108 (2001).

[16] W. Leo, Techniques for Nuclear and Particle Physics (Springer-Verlag, New York, 1987).

[17] M. Cummings, "Investigating Proton Spin Structure: A Measurement of $g_{2}^{p}$ at Low $Q^{2 ",}$ Ph.D. Thesis, https://wm1693.app.box.com/s/dsya4olvfo891h73t415/ file/54358033457, (The College of William \& Mary, May 2016).

[18] M. Iodice et al., "The $\mathrm{CO}_{2}$ Gas Cherenkov Detectors for the Jefferson Lab Hall-A Spectrometers", Nuclear Instruments and Methods in Physics Research A, 223 (1998).

[19] V. Sulkosky, "Data Acquisition for the Hall A High Resolution Spectrometers During 12 GeV", https://wiki.jlab.org/tegwiki/images/7/7d/HallA_ hrs_daq.pdf, 2014.

[20] N. Liyanage, Optics Calibration of the Hall A High Resolution Spectrometers Using the New Optimizer, tech. rep. JLab-TN-02-012 (Jefferson Lab, 2002).

[21] D. Nguyen, Summary of Local Efficiency of the Cherenkov at Trigger Level, https://hallaweb.jlab.org/dvcslog/Xgt2/171005_114948/XGT2_09_27_ summary_cer_eff.pdf.

[22] D. Meekins, private communication, Nov. 2018.

[23] D. Nguyen, "Target density determination through elastic scattering from ${ }^{3} \mathrm{He}$ in experiment E08014", unpublished, Mar. 2016.

[24] M. Berz, NSCL Technical Report, tech. rep. MSUCL-977 (Michigan State, 1995).

[25] L. Mo and Y. S. Tsai, "Radiative Corrections to Elastic and Inelastic eP and $\mu \mathrm{P}$ Scattering", Review of Modern Physics, 205 (1969).

[26] S. Stein et al., "Electron scattering at $4^{\circ}$ with energies of 4.5-20 GeV*", Physical Review D 12, 1884 (1975).

[27] H. Yao, "Precision Measurement of Longitudinal and Transverse Response Functions of Quasi-Elastic Electron Scattering in the Momentum Transfer Range 0.55 $\mathrm{GeV} / \mathrm{c} \leq q \leq 1.0 \mathrm{GeV} / \mathrm{c} "$, Ph.D. Thesis, https://digital.library.temple edu/digital/collection/p245801coll10/id/152421/, (Temple University, Jan. 2012).

[28] K. Slifer, "Spin Structure of ${ }^{3} \mathrm{He}$ and the Neutron at Low $\mathrm{Q}^{2}$ : A Measurement of the Extended GDH Integral and the Burkhardt Cottingham Sum Rule.", Ph.D. Thesis, https : / /wm1693.app.box.com/s/dsya4olvfo891h73t415/ file/31449285934, (Temple University, Nov. 2003).

[29] A. Amroun et al., "3 $\mathrm{H}$ and ${ }^{3} \mathrm{He}$ electromagnetic form factors", Nuclear Physics A 579, 596 (1994).

[30] G. Lafferty and T. Wyatt, "Where to Stick Your Data Points: The Treatment of Measurements within Wide Bins", Nuclear Instruments and Methods in Physics Research Section A 355, 541 (1995). 
[31] S. Li, Optics Calibration for ep, https://hallaweb.jlab.org/wiki/index php/File:Optics_Calibration_for_ep_(Round_1).pdf.

[32] D. Higinbotham, private communication, Oct. 2018.

[33] D. Nguyen, "The Isospin Dependence of Short Range Correlations through Inclusive Electron Scattering from ${ }^{40} \mathrm{Ca}$ and ${ }^{48} \mathrm{Ca}$ ", Ph.D. Thesis, https://libraetd lib.virginia.edu/public_view/k35694706, (University of Virginia, Nov. 2018).

[34] I. Nakagawa et al., "Measurement of the Elastic Magnetic Form Factor of ${ }^{3} \mathrm{He}$ at High Momentum Transfer", Physical Review Letters 86, 5446 (2001).

[35] H. Collard et al., "Elastic Electron Scattering from Tritium and Helium-3", Physical Review 138, B57 (1965).

[36] D. Beck et al., "Triton Form Factor from 0.29 to $1.00 \mathrm{fm}^{-2}$ ", Physical Review C 25, 1152 (1982).

[37] D. Beck et al., "Tritium Form Factors at Low q", Physical Review C 30, 1403 (1984).

[38] F. P. Juster et al., "Tritium Electromagnetic Form Factors", Physical Review Letters 55, 2261 (1985).

[39] D. Beck et al., "Isoscalar and Isovector Form Factors of ${ }^{3} \mathrm{H}$ and ${ }^{3} \mathrm{He}$ for $Q$ below $2.9 \mathrm{fm}^{-1}$ from Electron-Scattering Measurements", Physical Review Letters 59. 1537 (1987).

[40] M. Bernheim et al., "Elastic Electron Scattering from Site at High Momentum Transfer", Lettere Al Nuovo Ciment 5, 431 (1972).

[41] J. S. McCarthy, I. Sick, and R. R. Whitney, "Electromagnetic Structure of the Helium Isotopes", Physical Review C 15, 1396 (1977).

[42] Z. M. Szalata et al., "Low-momentum-transfer elastic electron scattering from ${ }^{3} \mathrm{He}$, Physical Review C 15, 1200 (1977).

[43] R. G. Arnold et al., "Elastic Electron Scattering from ${ }^{3} \mathrm{He}$ and ${ }^{4} \mathrm{He}$ at High Momentum Transfer", Physical Review Letters 40, 1429 (1978).

[44] J. M. Cavedon et al., "Magnetic Form Factor of ${ }^{3} \mathrm{He}$ ", Physical Review Letters 49, 986 (1982).

[45] P. C. Dunn et al., "3He magnetic form factor ", Physical Review C 27, 71 (1983).

[46] C. R. Otterman et al., "Elasti Electron Scattering from ${ }^{3} \mathrm{He}$ and ${ }^{4} \mathrm{He}$ ", Nuclear Physics A 436, 688 (1984).

[47] A. Amroun et al., "Isospin Separation of Three-Nucleon Form Factors", Physical Review Letters 69, 253 (1992).

[48] I. Sick, "Model-Independent Nuclear Charge Densities from Elastic Electron Scattering", Nuclear Physics A 218, 509 (1973).

[49] F. James, Minuit Function Minimization and Error Analysis Reference Manual, https://root.cern.ch/download/minuit.pdf.

[50] D. Higinbotham et al., Bias-Variance Trade-off and Model Selection for Proton Radius Extractions, https://arxiv.org/abs/1812.05706. 
[51] H. Akaike, "A New Look at the Statistical Model Identification", IEEE Transactions on Automatic Control 19, 716 (1974).

[52] G. Schwarz, "Estimating the Dimension of a Model", The Annals of Statistics 6. 461 (1977).

[53] R. E. Kass and A. E. Raftery, "Bayes Factors", Journal of the American Statistical Association 90, 773 (1995).

[54] A. Rohatgi, Web Plot Digitizer, https://automeris.io/WebPlotDigitizer/.

[55] L. S. Myers, D. W. Higinbotham, and J. R. Arrington, E12-14-009: Ratio of the electric form factor in the mirror nuclei ${ }^{3} \mathrm{He}$ and ${ }^{3} \mathrm{H}$, https://arxiv.org/pdf/ 1408.5283.pdf.

[56] L. E. Marcucci et al., "Electromagnetic Structure of Few-Nucleon Ground States", Journal of Physics G: Nuclear and Particle Physics 43, 1 (2016).

[57] D. Higinbotham et al., "Measurement of the ${ }^{3}$ He Magnetic Form Factor in the Region of the First Diffraction Minimum using Polarization Observables", unpublished, May 2000. 